\title{
ICAR 2011: Abstracts
}

\author{
CO 01 \\ THE LOWEST X4 GENO2PHENO-FALSE POSITIVE RATE IS \\ ASSOCIATED WITH GREATER CD4-DEPLETION \\ M.M. Santoro $*^{l}$, D. Armenia ${ }^{I}$, L. Fabeni ${ }^{2}$, M. Santoro ${ }^{I}$, C. Gori ${ }^{2}$, F. Forbici ${ }^{2}$, \\ V. Cento ${ }^{l}$, V. Svicher ${ }^{l}$, A. Bertoli ${ }^{l}$, L. Dori ${ }^{l}$, M. Andreoni ${ }^{l}$, P. Narciso ${ }^{2}$, \\ A. Antinori ${ }^{2}$, F. Ceccherini-Silberstein ${ }^{I}$, C.F. Perno ${ }^{I, 2}$ \\ ${ }^{I}$ University of Tor Vergata, Rome, Italy; ${ }^{2}$ I.N.M.I. "L. Spallanzani", Rome, \\ Italy
}

Background: CXCR4 phenotype has been associated with increased severity of HIV disease, higher viral load, and decreased CD4 T-cell counts. We thus evaluated whether a genotypic analysis of coreceptor tropism impacts on HIV-related markers such as CD4-cell counts.

Methods: 960 HIV-1 B subtype infected patients (all maravirocnaive) with an available V3 sequence were analyzed. Tropism determination was performed by using Geno2Pheno algorithm. All V3 mutations, including the mutations used for tropism prediction (Sing $\mathrm{T}$ et al., Antiviral Therapy 2007), with prevalence $>1 \%$ were considered. The associations of CD4 cell counts with false positive rate (FPR) ranges and V3 mutations were evaluated by MannWhitney test, Chi-squared test for trend and Fisher exact test, as appropriate. The Benjamini-Hochberg method was used to identify results that were statistically significant in the presence of multiplehypothesis testing. A false discovery rate of 0.05 was used to determine statistical significance.

Results: Overall, $26 \%$ of patients (either drug-naive or drug-experienced) were infected by X4 tropic viruses (using the classical 10\% FPR cutoff). Among patients with available CD4 cell-counts at V3 genotypic test, 321 were drug-naive and 490 drug-experienced (median [IQR] CD4-cell counts: 331 [201-448] vs. 282 [138-422] cells $\left./ \mathrm{mm}^{3}, \quad \mathrm{p}=0.004\right)$. CD4 cell-count $<200$ cells $/ \mathrm{mm}^{3}$ was observed in 80 drug-naive patients and in 169 drug-experienced patients. For different CD4 cell count ranges, FPR $<2 \%$ was mostly associated, in experienced patients, with CD4 cell-counts $<200$ cells $/ \mathrm{mm}^{3}$ ( $<200$ vs. $200-350$ vs. $350-500$ vs. $>500$ cells/ $\mathrm{mm}^{3}: 21.3$ vs. 7.6 vs. 9.0 vs. $8.0 \%$, respectively, $\left.\mathrm{p}=0.0005\right)$. This finding was also observed in drug-naive patients, even if with less significance ( 11.3 vs. 6 vs. 2.6 vs. $1.5 \%$, respectively, $\mathrm{p}=0.040)$. A deeper analysis showed that patients with FPR $<2 \%$ (both naive and drug-experienced) carried a significantly greater risk of having CD4 counts $<200$ cells $/ \mathrm{mm}^{3}$ (see figure). Among all V3 mutations analyzed (87 in naive patients and 88 in experienced patients), in drug-experienced patients the X4-related S11R was the only mutation found associated with CD4 cell count $<200$ cells $/ \mathrm{mm}^{3}$ ( 17.8 vs. $5.6 \%$ in $<200$ vs. $>200$ CD4, respectively, p $<0.001$ ). This significance was confirmed after correction for multiple comparisons.

Conclusions: Within the context of genotypically-defined CXCR4 tropism, FPR $<2 \%$ and the X4-related mutation S11R are associated with low CD4 rank, and thus may be related with a greater risk of disease progression. These findings suggest that FPR, beyond tropism prediction, can be used also as a marker to evaluate the immunological status of HIV-1 infected patients.

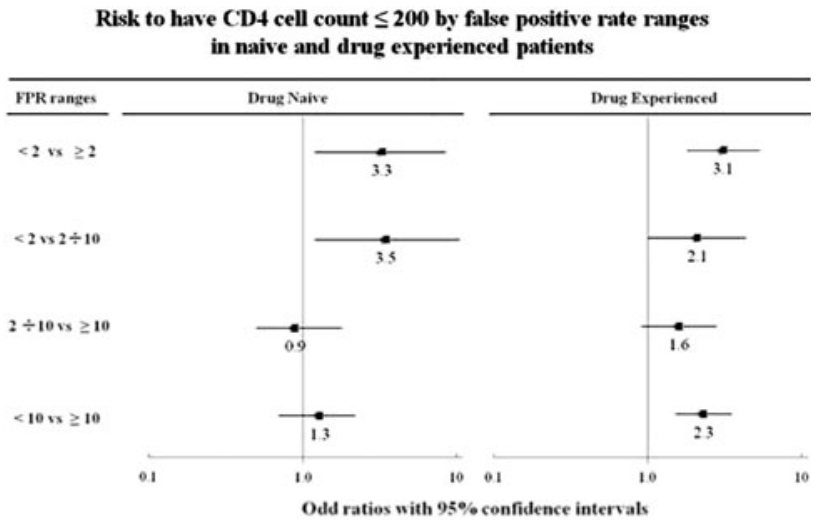

CO 02

A GLYCOMIMETIC COMPOUND INHIBITS DC-SIGN MEDIATED HIV INFECTION IN CELLULAR AND CERVICAL EXPLANT MODELS

A. Berzi* ${ }^{1}$, S. Sattin ${ }^{2}$, J. Reina ${ }^{2}$, M. Sanchez-Navarro ${ }^{3}$, J. Rojo ${ }^{3}$, D. Trabattoni ${ }^{1}$, P. Antonazzo ${ }^{4}$, I. Cetin $^{4}$, A. Bernardi ${ }^{2}$, M. Clerici $^{5}$

${ }^{I}$ Department of Clinical Science L. Sacco, University of Milan, Milan, Italy; ${ }^{2}$ Department of Organic and Industrial Chemistry, University of Milan, Milan, Italy; ${ }^{3}$ Instituto de Investigazione Químicas, University of Sevilla, Sevilla, Spain; ${ }^{4}$ Unit of Obstetrics and Gynecology, Hospital L. Sacco, Milan, Italy; ${ }^{5}$ Air of Immunology, University of Milan, Milan, Italy

Background: Development of topical microbicides and entry inhibitors is a promising approach to prevent sexually transmitted HIV infection. Mucosal dendritic cells (DCs) internalize HIV through DCSIGN and, on arrival to lymphoid tissues, transmit the virus in trans to CD4 T lymphocytes, promoting HIV dissemination. DC-SIGN, thus, is a potential therapeutic target and the inhibition of HIV interaction with DC-SIGN may efficiently prevent the early stages of HIV infection. The DC-SIGN ligand is the HIV gp120 high mannose glycan Man9. We synthesized structural analogues of this glycan (using polyvalent presentations of di- and tri-mannoside mimics) in the attempt to compete with binding of DC-SIGN to HIV gp120.

Methods: Different experimental models were designed: (1) inhibition of trans infection was assessed by using B-THP-1/DC-SIGN cells. Ability of compounds to block lab and primary HIV-1 strains transmission to CD4 T cells was evaluated; (2) human endocervical explant tissues were treated with the compounds and then exposed to different HIV-1 strains in a non polarised manner. Infection was determined by measuring p24 levels in co-culture and in explant culture supernatants; (3) cytokines and chemokines production following stimulation of monocyte-derived DCs was analyzed. Toxicity of the compounds was evaluated in cellular and tissue models.

Results: One of the compounds tested, a tetravalent dendron containing four copies of a linear trimannoside mimic (compound 12), almost completely ( $>98 \%$ ) abrogated the transmission of R5- and 
X4-tropic HIV-1 lab strains and primary isolates to CD4 T cells at $100 \mu \mathrm{M}$. Notably the antiviral effect persisted up to $12 \mathrm{~h}$ after compound removal. Compound 12 also prevented, in a dose-response manner, HIV-1 infection of human cervical tissues under conditions which mimic compromised epithelial integrity. Treatment with this compound significantly increased MIP-1alpha production as well. Toxicity of compound 12 was neglectable at the highest concentration tested in infection assay.

Conclusion: Compound 12, a tetravalent dendron presenting trimannoside mimic, is endowed with a potent anti HIV activity independent of viral tropism. The activity is long-lasting and observed both when CD4 $\mathrm{T}$ lymphocytes or cervical explants are used. Competition with the binding of HIV to DC-SIGN and stimulation of MIP1alpha production both contribute to such activity. This compound is potentially suitable for development as a vaginal microbicide.

\section{CO 03}

PROVIRAL HIV CHANGES CORRELATE WITH CD4+ CD45RA+ T-CELL RECOVERY IN PATIENTS RECEIVING RALTEGRAVIRINCLUDING REGIMENS

N. Gianotti ${ }^{\prime}$, M. Malnati ${ }^{2}$, L. Galli ${ }^{1}$, A. Galli ${ }^{1}$, M. Tolazzi $i^{3}$, G. Cassina $^{2}$, S. Nozzal ${ }^{I}$, L. Della Torre ${ }^{I}$, A. Castagna ${ }^{l}$, G. Scarlatti ${ }^{3}$

${ }^{I}$ Department of Infectious Diseases, San Raffaele Scientific Institute, Milan, Italy; ${ }^{2}$ Human Virology Unit, San Raffaele Scientific Institute, Milan, Italy;

${ }^{3}$ Viral Evolution and Transmission Unit, San Raffaele Scientific Institute,

Milan, Italy

Background: There is limited knowledge on the effects of raltegravir (RAL)-including regimens on proviral HIV load and whether proviral changes in $\mathrm{CD} 4+\mathrm{T}$ cells may provide additional information over RNA changes in treated patients.

Methods: Patients receiving new RAL-including regimens after failure of a previous therapy were prospectively followed. Those with samples stored at baseline and after at least 48 weeks of therapy were included in the study. PBMC were analyzed by a FACS-can flow cytometer (Becton-Dickinson). CD4+ $\mathrm{T}$ cells, positively purified from PBMC (Miltenyi), were lysed and HIV DNA quantified with real time PCR. Slopes were estimated by univariable linear regression analysis; Spearman correlation coefficients (r) were also calculated.

Results: Thirty-eight patients, $84 \%$ males, median (IQR) age 48 (45-53) years, fulfilled inclusion criteria. At baseline (BL) their CD4+ counts, HIV RNA, HIV DNA were $250(165-355) / \mathrm{mcL}, 3.97$ (3.76-4.97) $\log _{10}$ copies/mL, and 5,090 (3,004-1,1074) copies/106 CD4+ cells, respectively. After a median follow-up of 24 (22-25) months, 35 (92.1\%) patients had $<50$ HIV RNA copies/mL, CD4+ cells increased by $176(98-278)$ cells/mcL and HIV DNA decreased by 2,983 (657-6,973) copies/106 CD4+ cells. Correlations between HIV RNA and DNA variations and immune and virological changes are reported in the table.

\begin{tabular}{|c|c|c|c|c|}
\hline & \multicolumn{2}{|c|}{$\begin{array}{l}\text { HIV RNA log } \\
\text { change }\end{array}$} & \multicolumn{2}{|l|}{$\begin{array}{l}\text { HIV } \\
\text { change }\end{array}$} \\
\hline & $\mathrm{r}$ & $\mathrm{p}$ & $\mathrm{r}$ & $\mathrm{p}$ \\
\hline HIV RNA log change & - & - & 0.55 & 0.0004 \\
\hline HIV RNA log slope & - & - & 0.5 & 0.001 \\
\hline BL HIV DNA $/ 10^{6}$ CD4+ T cells & -0.65 & $<\mathbf{0 . 0 0 0 1}$ & -0.89 & $<0.0001$ \\
\hline BL HIV RNA & -0.98 & $<\mathbf{0 . 0 0 1}$ & -0.5 & 0.001 \\
\hline CD4+ \# change & -0.49 & 0.002 & -0.52 & 0.001 \\
\hline CD4+ \# slope & -0.45 & 0.004 & -0.54 & 0.0004 \\
\hline BL CD4+ \# & 0.44 & 0.006 & 0.06 & 0.73 \\
\hline $\mathrm{CD} 4+\%$ change & -0.16 & 0.32 & -0.28 & 0.08 \\
\hline
\end{tabular}

Table a continued

\begin{tabular}{|c|c|c|c|c|}
\hline & \multicolumn{2}{|c|}{$\begin{array}{l}\text { HIV RNA log } \\
\text { change }\end{array}$} & \multicolumn{2}{|l|}{$\begin{array}{l}\text { HIV } \\
\text { change }\end{array}$} \\
\hline & $\mathrm{r}$ & $\mathrm{p}$ & $\mathrm{r}$ & $\mathrm{p}$ \\
\hline $\mathrm{BL} \mathrm{CD} 4+\%$ & 0.45 & 0.004 & 0.2 & 0.22 \\
\hline $\mathrm{CD} 8+\%$ change & 0.08 & 0.62 & 0.31 & 0.06 \\
\hline $\mathrm{BL} \mathrm{CD} 8+\%$ & -0.07 & 0.66 & -0.15 & 0.36 \\
\hline CD4+ CD45RA+ change & -0.14 & 0.41 & -0.38 & 0.02 \\
\hline BL CD4+ CD45RA+ & 0.37 & 0.02 & 0.15 & 0.36 \\
\hline CD4+ CD45RA-change & -0.2 & 0.24 & -0.05 & 0.77 \\
\hline BL CD4+ CD45RA- & 0.48 & 0.002 & 0.18 & 0.29 \\
\hline CD8+ HLA-DR+ CD38+ change & 0.41 & 0.01 & 0.18 & 0.29 \\
\hline BL CD8+ HLA-DR+ CD38+ & -0.34 & 0.04 & -0.16 & 0.34 \\
\hline
\end{tabular}

Conclusions: During treatment with RAL-including regimen, the decrease of HIV RNA, but also of proviral DNA load in purified CD4+ cells, correlates with recovery of CD4+ cells. Changes of HIV DNA but not HIV RNA, correlate with CD4+ CD45RA+ T-cells recovery, suggesting a decrease of the viral reservoir in the circulating naive/terminal effector CD4+ cells. The decrease of CD8+ HLA-DR+ CD38+ cells indicates also an amelioration of their activation status, which correlates with HIV RNA variations only. These results drive to further investigate the nature of the CD4+ T cell speared by HIV infection under RAL treatment.

\section{CO 04}

A STUDY OF HIV CORECEPTOR TROPISM IN PAIRED PLASMA, PBMC AND CEREBROSPINAL FLUID ISOLATES FROM NAÏVE SUBJECTS

C. Andreoni ${ }^{1}$, S.G. Parisi ${ }^{2}$, L. Sarmati ${ }^{3}$, A.R. Buonomini ${ }^{3}$, G. D'Ettorre ${ }^{1}$,

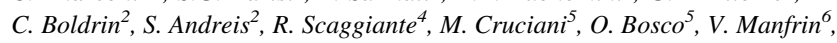
C. Mengoli ${ }^{2}$, G. Palü ${ }^{2}$, V. Vullo ${ }^{1}$, M. Andreoni ${ }^{3}$

${ }^{I}$ Cattedra di Malattie Infettive, La Sapienza, Rome, Italy; ${ }^{2}$ Dipartimento di istologia, microbiologia e biotecnologie, università di Padova, Padua, Italy; ${ }^{3}$ Cattedra di Malattie Infettive, Tor Vergata, Rome, Italy; ${ }^{4}$ Unità di Malattie Infettive, Ospedale di Padova, Padua, Italy; ${ }^{5}$ Centro di Medicina Preventiva, ULSS 20, Verona, Italy; ${ }^{6}$ Unità di malattie Infettive, Vicenza, Italy

A survey of HIV coreceptor usage in cerebrospinal fluid (CSF), peripheral blood mononuclear cells (PBMCs) and plasma in naïve seropositive patients was conducted. One hundred of patients were enrolled, of these 36 had a primary or recent infection (P-RI), 31 an early chronic infection ( $>350 \mathrm{CD} 4$ cells) (ECI) and 33 a late chronic infection (LCI). Seventy-seven patients harboured the R5 virus in plasma and had a significantly higher median and percentage of CD4+ T cells compared to patients with X4 virus (437 and 281 cells $/ \mu 1$, $\mathrm{p}=0.0086 ; 20.6$ and $18.6 \%$; respectively). The $\mathrm{X} 4$ strain was detected more frequently in patients with LCI than with P-RI or ECI (39.3, 19.4 and $9.6 \%$, respectively, $\mathrm{p}=0.0063)$. PBMC and plasma tropism was concordant in 90 patients, and 73 had the R5 strain. Among discordant patients, four had the R5 virus in their plasma and the $\mathrm{X} 4$ virus in PBMCs; six showed the opposite profile. Plasma, PBMC and CSF tropism determinations were concordant in $26 / 33$ patients (21 R5 and $5 \mathrm{X} 4$ ). The tropism was discordant in 5/33 patients, with the $\mathrm{X} 4$ virus in plasma and R5 in CSF; the HIV tropism in PBMCs was X4 in 3 patients. The remaining 2/33 patients had the $\mathrm{R} 5$ virus in plasma and PBMCs and the $\mathrm{X} 4$ virus in CSF; one of these patients had PRI. The discordant tropism in CSF and blood may have implications for CCR5 antagonist use in patients with limited response to antiretroviral therapy (ART) or in responding patients evaluated for simplification of treatment. 


\section{CO 05}

ACCUMULATION OF DEFECTIVE HI-1 VARIANTS IN A SUBTYPE SLOW-PROGRESSOR WITH HIGH RT-SPECIFIC CD8+ MEMORY T-CELL RESPONSE

S. Paolucci ${ }^{*}$, A. Foli ${ }^{2}$, S. Calarota ${ }^{1}$, R. Gulminetti ${ }^{3}$, F. Baldanti ${ }^{1}$

${ }^{I}$ SS Virologia Molecolare SC Virologia e Microbiologia, Fondazione IRCCS Policlinico San Matteo, Pavia, Italy; ${ }^{2}$ Lab. Biotecnologie Fondazione IRCCS Policlinico San Matteo, Pavia, Italy; ${ }^{3}$ Malattie Infettive Fondazione IRCCS Policlinico San Matteo, Pavia, Italy; ${ }^{4}$ SS Virologia Molecolare SC Virologia e Microbiologia, Fondazione IRCCS Policlinico San Matteo, Pavia, Italy

Objective: The peculiar viral population in a slow progressor carrying CRF02-AG HIV-1 virus with several stop codons in the RT gene was analyzed. A role for the T-cell response in the selection of replication-deficient variants was investigated.

Methods: The proportion of mutant and wild-type RT sequences was determined by clonal analysis of HIV-1 DNA and RNA from blood samples and peripheral blood mononuclear cell (PBMC) culture supernatants. In addition, recombinant HIV-1 strains were generated by reverse genetics to evaluate the replicative capacity of different RT variants in PBMC cultures. The HIV-1-specific memory CD8+ T-cell response was investigated using a peptide-based cultured ELISPOT assay.

Results: HIV-1 RNA and DNA sequences in PBMC cultures showed a mixture of stop codons (RTSTOP), recombinant forms (RTRF), and full length (RTFL) strains. The proportion of HIV-1 RNA sequences with truncated RT gene fluctuated in plasma over time was $0 \%$ in $2005,100 \%$ in 2007 and $8.3 \%$ in 2010 , while it was constant (96.5-100\%) in proviral DNA. Reconstituted RTSTOP strains were unable to replicate in PBMC. However, RTFL strains could transcomplement the loss of function of RTSTOP variants. The cultured ELISPOT showed a sustained response to a Pol region downstream from the last stop codon. A more detailed analysis of the Pol region encompassing the detected stop codons showed a strong response to a peptide at the end of the RT region containing stop codons (positions 206-220) and including the last stop codon (212).

Conclusion: In vivo selection of multiple stop codons in the RT gene resulted in the accumulation of replication-defective virus strains. Nevertheless, the observed assembly and release of defective viral particles in plasma was probably the result of viral protein complementation between replication-competent and replicationincompetent HIV-1 variants. Interestingly, the divergence in the proportion of RTSTOP and RTFL variants as well as in the pattern of mutations to antiretroviral drug resistance between HIV-1 plasma RNA and circulating PBMC proviral DNA sequences, suggested the possibility that replication-competent and -incompetent virus particles might be assembled and released from lymphatic tissues through a trans-complementation mechanism, while circulating lymphocytes expressing full-length RT might be negatively selected for by specific $\mathrm{T}$-cell response, possibly slowing progression to AIDS in this patient. These results suggest a role for a peptidespecific immunologic response in the positive selection of cells expressing the truncated HIV-1 RT and the accumulation of replication-deficient viral variants in plasma. The antigen-specific CD8+ $\mathrm{T}$-cell response could be exploited to redirect the response to HIV-1 infection toward in vivo selection of viral variants with reduced or abolished pathogenicity.

\section{CO 06}

THE ADAR EDITING ENZYMES ARE NOVEL HIV-1 PROVIRAL FACTORS

M. Doria ${ }^{2}$, S. Tomaselli ${ }^{2}$, F. Neri ${ }^{2}$, S.A. Ciafre ${ }^{\text {, M.G. Farace }}$, A. Gallo ${ }^{2}$, A. Michienzi* ${ }^{I}$

${ }^{I}$ Università di Roma "Tor Vergata", Rome, Italy; ${ }^{2}$ Ospedale Bambin Gesù, Rome, Italy
One of the best-characterized mechanisms of RNA editing is the conversion of adenosine into inosine (A-to-I) mediated by the Adenosine DeAminase enzymes or ADARs that act on doublestranded RNA. In mammals, three different ADAR enzymes have been identified: ADAR1, ADAR2, and ADAR3 [1]. Inosine acts as guanosine during both splicing and translation events, therefore A-toI editing within pre-mRNA can alter both splicing patterns and amino acid sequence with important consequences in the final function of the coded protein [1]. ADARs can target viruses, as suggested by numerous reports showing A-to-I changes identified in viral genomes or transcripts that are consistent with editing mediated by these enzymes [1]. So far little effort has been dedicated to testing the involvement of ADARs in the life cycle of the human immunodeficiency virus type 1 (HIV-1). HIV-1 RNAs contain several double stranded regions, some of them critical for the different steps of the viral life cycle such as the Rev responsive element (RRE), transactivation responsive element (TAR), and dimerization domain (DIS) [2] that could be possible ADARs substrates. Recently we provided evidence of a role for ADAR1 and ADAR2 in the regulation of some critical steps of the HIV-1 life cycle [3, 4]. We demonstrated that over-expressed ADAR1 and ADAR2 strongly increase the overall accumulation of HIV-1 proteins in producer cells independently of their editing activity. Through their RNA editing activity, both enzymes enhance the release of progeny virions from the producer cells. Of note, only the ADAR1-mediated editing increases the infectious potential of the virus. Finally, we demonstrated that both enzymes edit adenosines within the $5^{\prime}$ UTR of viral transcripts, but only ADAR1 edits specific adenosines within the Rev and Tat coding sequence. Therefore, ADAR2 and ADAR1 share some but not all the functions that positively regulate HIV-1 replication. Importantly, down-regulation of ADAR2 in Jurkat cells significantly impairs viral replication. Future investigations are necessary to understand the impact of ADARs in the HIV-1 life cycle. This study, by providing further insight into the role of the ADAR enzymes in stimulating the sequence diversification and replication of HIV-1, will possibly help the disclosure of novel targets for antiviral intervention.

\section{CO 07}

SIALON PROJECT (2008-2010): HIV PREVALENCE, UNDIAGNOSED INFECTIONS, DRUG USE AND UNPROTECTED ANAL INTERCOURSE AMONG MSM ATTENDING GAY VENUES IN SIX EUROPEAN CITIES (BARCELONA, BRATISLAVA, BUCHAREST, LJUBLJANA, PRAGUE AND VERONA)

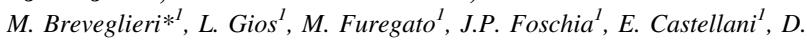
Ramarli $^{2}$, P. Coato $^{2}$, M. Mirandola ${ }^{I}$

${ }^{1}$ Regional Centre for Health Promotion, Regione Veneto, Verona, Italy; ${ }^{2}$ Dipartimento Patologia e Diagnostica, Università di Verona, Verona, Italy

Background: HIV and STI prevalence reported among men having sex with men (MSM) has risen in recent years in European countries concomitantly to an increase in unsafe sexual practices. The use of psychoactive drugs is an important factor linked to risk behaviour and risk of HIV transmission.

Methods: A multi-centre biological and behavioural cross-sectional survey was designed to obtain a "one time" estimation of the HIV prevalence in MSM attending gay venues through the use of selfadministered questionnaires (UNGASS and ECDC indicators) and oral fluid (OF) samples (valid samples: 2.287) in 2008 and 2009. Data were collected in six cities of Southern and Eastern Europe through a (TLS) Time-Location-Sampling method. HIV prevalence is based on OF samples. Drug use is defined as use of at least one of the following: Poppers, Ecstasy, Viagra, Hashish/Marijuana, Cocaine, Amphetamine. Unprotected Anal Intercourse (UAI) is defined as anal intercourse without condom use.

Results: HIV prevalence was $17.0 \%$ in Barcelona, $11.8 \%$ in Verona, $6.0 \%$ in Bratislava, $5.1 \%$ in Ljubljana, $4.6 \%$ in Bucharest and $2.6 \%$ 
Prague. $56 \%$ of all HIV+ MSM were not aware of their serostatus. This figure was almost $80 \%$ in Bucharest and Ljubljana, $57.1 \%$ in Verona and Prague, and lower than 50\% only in Barcelona (44.6\%). In the whole sample the percentage of MSM using drugs during the last sexual intercourse among HIV+ subjects was higher $(43.11 \%)$ than in HIV - $(21.84 \%)(\mathrm{P}<0.000)$. HIV+ MSM were more likely to use drugs than HIV $-(\mathrm{P}<0.05)$ in four cities: Prague, Bratislava, Barcelona, Verona. No significant differences occurred in Bucharest $(\mathrm{P}=0.260)$ and Ljubljana $(\mathrm{P}=0.268)$. MSM reporting UAI last time they had sex were more likely to use drugs $(28.59 \%)$ than MSM not reporting UAI $(19.71 \%) \quad(\mathrm{P}<0.000)$; similar proportion $(\mathrm{P}<0.05)$ occurred in Prague, Barcelona, Ljubljana, with the exception of Bratislava $(\mathrm{P}<0.161)$, Bucharest $(\mathrm{P}<0.104)$, Verona $(\mathrm{P}<0.187)$.

Conclusions: HIV prevalence was higher in Southern European cities than elsewhere. Data seems to suggest that drug use before or during sex occurred more frequently among HIV+ MSM rather than HIVand it is related to a higher level of UAI. Data underlines the need of specific prevention programmes targeted on drug use. Outreach prevention programmes promoting HIV testing are also needed in order to promote HIV testing and detect undiagnosed infections.

Project co-funded by the European Commission under the Public Health Programme 2003-2008.

\section{CO 08}

2007-2010: DAY CARE ACCOMMODATION FOR PLWHA IN LOMBARDY REGION

L. Rancilio* ${ }^{\prime}$, A. Grassini ${ }^{I}$, G. Gaiera ${ }^{I}$

${ }^{I}$ Coordinamento Case Alloggio per persone con HIV/AIDS della Lombardia (C.R.C.A. Lombardia), Milan, Italy

Since 2007 every summer the "Coordinamento Case Alloggio per persone con HIV/AIDS della Lombardia (C.R.C.A. Lombardia)" has been collecting data about its guests and using an evaluation form pointed up by C.E.R.C.A.Re. project. In the out-of-hospital facilities in Lombardy Region there are 149 bedplaces in Highly Integrated Health-Social Care Family Homes and 65 bedplaces in Low-Intensity Assistance Family Homes plus 58 places for guests only during the day in some family homes and in 2 Daily Centres. Daily facilities are almost not available in Italy. Since July 2007 till June 2010590 people had been accepted in facilities in Lombardy. 109/590 (18.5\%) have had periods of care in Daily Centres: 85/454 men, 24/136 women. $3.7 \%$ had age $19-35,70.6 \% 36-50,20.2 \%$ 51-65, while the ultra-65 years represented $5.5 \%$. People cared in daily facilities are carriers of health and social problems such as mental disorders and physical disabilities, even if less than that observed in the total population surveyed: respectively 31.2 versus $48.3 \%$ and 30.3 versus $37.6 \%$. 16.5 versus $13.4 \%$ were active drug users, 10.1 versus $215 \%$ had methadone replacement therapy. 14.7 versus $15.1 \%$ had alcohol dependence. 81/109 (74.3\%) persons accepted in daily facilities versus $451 / 590(76.4 \%)$ were assessed with the instrument developed by 2007 C.E.R.C.A.Re. project. People entering and leaving facilities before the application of the instrument every summer have not been evaluated by. The people in Daily Centres reach better evaluations in many indicators than average of the total sample. On a scale of 0-3 scores, $64.5 \%$ people in daycare facilities achieved a score of 2 or 3 in physical condition items versus $54.5 \%$ total. Also they seem to be able to put into play some more personal resources to cope with the disease (59.6 versus $53.5 \%$ ). Obviously the presence of adequate housing is first of all what makes possible the daily attendance, rather than having to be accommodated in a residential service, but also it's important the supporting presence of affective and relational network available for $61.5 \%$ of the Day care guests versus $44.8 \%$ of the total. The great difficulty to gain employment perspectives hamper the possibilities of people's autonomy as in Family homes as in Day care centres. The location of the guests in the quadrants of the
C.E.R.C.A.Re. graphic instrument reflects the variety of the population to whom residential and semi-residential services are addressed and the complex clinical and social needs and care to which they respond.

\section{CO 09}

IN A HAART NON-ADHERENT POPULATION, WOMEN REPORT SIGNIFICANTLY POORER ADHERENCE BEHAVIORS, HIGHER SYMTPOMS RATES, AND WORSE HEALTH STATUS WHEN COMPARED TO MEN

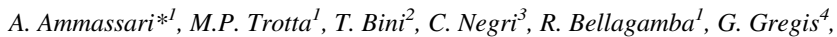
M. Tettoni ${ }^{5}$, A. Capetti $^{6}$, C. Tommasi ${ }^{1}$, L. Trentini ${ }^{5}$, B. Argenteri ${ }^{6}$, M. Zaccarelli ${ }^{1}$, L. Comi ${ }^{2}$, S. Leone ${ }^{4}$, L. Dori ${ }^{7}$, P. Nasta ${ }^{8}$, A. Antinori ${ }^{l}$ ${ }^{1}$ INMI "L. Spallanzani", Rome, Italy; ${ }^{2}$ Ospedale San Paola, Milan, Italy; ${ }^{3}$ Università di Milano, Milan, Italy; ${ }^{4}$ Ospedale di Bergamo, Bergamo, Italy; ${ }^{5}$ Università di Torino, Turin, Italy; ${ }^{6}$ Ospedale Luigi Sacco, Milan, Italy; ${ }^{7}$ Università Tor Vergata, Rome, Italy; ${ }^{8}$ Ospedali Riuniti di Brescia, Brescia, Italy

Background: HAART non-adherence is still a relevant phenomenon among HIV-positive persons and may be attributable to patient characteristics and treatment-related variables. So far, differences in reasons for non-adherence have not been investigated in women and men treated with HAART. Compared to HIV-positive men, women are known to report more symptoms, present higher depression rates, and personify profoundly diverse family and societal roles. The present analysis aimed to identify gender differences regarding adherence behaviors and its predictive variables among HAART non-adherent persons.

Methods: The SupportHIV study is a randomized open-lable trial aimed to test the efficacy of a multidimensional intervention on HAART adherence in a non-adherent patient population. Participants were asked to fill a questionnaire which investigates different types of non-adherence (percentage of therapy taken in the last month; missed doses in the last week; timing deviation; interruption in drug refill over last 3 months, and treatment interruptions over the last month), symptoms, depression, and adherence barriers. Inclusion criterion: self-reported non-adherence. Socio-demographic variables, HIV- and HAART-related information were collected.

Results: Of the 365 non-adherent patients included in the trial, $27.4 \%$ were female gender. Overall median age was 45 (IQR 40-49) and route of HIV-infection was intravenous drug use in $38.5 \%$, heterosexual and homosexual intercourse in 29.4 and $27.5 \%$, respectively. The regimen was a first-line treatment in $18.6 \%$ and delivered BID in $42 \%$ of cases. HAART included PI/b in $39.9 \%$, NNRTI in $10.2 \%$, only NRTI in $15.2 \%$. When compared with men (M), women (F) reported a significantly higher percentage adherence $<95 \%$ in the last months ( $\mathrm{M} 36.6 \%$ vs. $\mathrm{F} 52 \% ; \mathrm{P}=0.006$ ) as well as treatment interruptions ( $\mathrm{M} 27.5 \%$ vs. $\mathrm{F} 40.4 \%$; $\mathrm{P}=0.02$ ). Women showed lower global health status (median [IQR] in M 80 [60-100], and in F 77 [50-90]; $\mathrm{P}=0.03$ ), and higher tendency for depressed mood (63.9\% of $\mathrm{F}$ were scored at CES-D, compared with $47.3 \%$ of $\mathrm{M}$ ). Among variables investigated in the questionnaire as potential reasons for drug interruption, $\mathrm{F}$ reported more frequently to have to take too big pills $(\mathrm{P}<0.001)$, to take pills with unpleased taste $(\mathrm{P}<0.001)$, to have already experienced previous side-effects $(\mathrm{P}=0.02)$. Finally, an higher symptoms score was found among $\mathrm{F}$ (mean value 16.4), when compared with $\mathrm{M}$ (mean value 12.3) $(\mathrm{P}=0.002$ at $t$ test). In particular, the following symptoms were reported more frequently among $\mathrm{F}$ than $\mathrm{M}$ : headache, fatigue, jaundice, gastric problems, nausea, bloating, and fat accumulation (all $\mathrm{P}<0.05$ at Chi-square).

Conclusions: In this population of HAART non-adherent persons, gender-stratified analysis demonstrated that women are more prone to non adherence deviations. Identification of gender-specific issues correlated with HAART non-adherence may help in better addressing specific intervention to improve adherence. 


\section{CO 10}

\section{ACCESS TO HIV TESTING IN ITALY}

A. Colucci $*^{1}$, P. Gallo ${ }^{1}$, S. D'Amato ${ }^{2}$, M. Oldrini ${ }^{3}$, L. Rancilio ${ }^{3}$, M.G. Pompa ${ }^{4}$, A.M. Luzi on behalf of the Study Group ${ }^{I}$

${ }^{I}$ Department of Infectious, Parasitic and Immune-Mediated Diseases, Italian National Institute of Health, Rome, Italy; ${ }^{2}$ National AIDS Center, Italian Institute of Health, Rome, Italy; ${ }^{3}$ Council for the fight against AIDS; ${ }^{4}$ Italian Ministry of Health

Introduction: The Italian National Institute of Health and the Council for the fight against AIDS have conducted a research which has been financed by the Ministry of Health. Aim was the assessment and implementation of intervention models aimed to increase the access to HIV tests.

Methodology: The survey has been conducted interviewing on the phone managers from 391 Clinical-Diagnostic Centers (CDC) and 216 Transfusion Centers (TC).

Results: The data analysis revealed that in $77.0 \%$ of the CDCs, HIV test was free whereas anonymity was guaranteed in $38.4 \%$ of cases, pre-test counseling was performed in $48.1 \%$ of cases and post-test counseling in $44.7 \%$. With regard to the window period indication, $27.8 \%$ of managers answered that the test should be carried out "6 months after exposure", 18.4\% answered "after 3 months" and $8.9 \%$ said "after a month". In $44.9 \%$ of cases, respondents provide different indications. Test result is given by the doctor in $33.9 \%$ of the CDCs and in $28.9 \%$ of cases by the health staff who took the blood for the HIV testing, during the counseling. In $32.7 \%$ of the CDC the test result is given in a sealed envelope by designated clinic staff or any acceptance personnel. With regard to HIV testing for children, in $26.1 \%$ of the CDC the test is preceded by a pre-test counseling and followed by a post-test counseling, having obtained parental consent. As for the blood TC, it was found that $26.4 \%$ of general population (not only donors) get an HIV test; $66.7 \%$ of TCs which took part in the study belong to an hospital; 199 centers $(92.1 \%)$ have always provided a pre-donation interview, while the informed consent to get both the consent and the signature of the person is offered in $189(87.6 \%)$ blood centers. In case of a positive HIV test result, $65.7 \%$ of the surveyed centers-which are all legally bound to not reveal personal details and test results-have contacted and invited the person to visit the TC for having medical care and other appropriate services.

Conclusions: The results shows that in the surveyed centers there are very different testing approach and methods, especially with regard to three main factors: anonymity, pre and post-test counseling and freeness. Given such an obvious lack of homogeneity, both national and local health authorities should give high priority in providing useful information to unify the methods of HIV testing access and availability, giving all the people in Italy, the same health care opportunities.

Study Group

Rossi G, Forum AIDS Italia-Lorenzetti D., ANLAIDS-Parisi R, San Benedetto al Porto-Stagnitta M e Camposeragna A, CNCASanvito L, CICA-Von Schlosser F, Biondi R, Nadir-Farinella M, Mario Mieli-Brunetti L, Ghibaudi J, Gruppo Abele-Errico M, Zumbo G, NPS-Prota P, Public Health Agency of Lazio (adviser, Italian National Institute of Health).

\section{CO 11}

HIV INFECTION AND MIGRANTS: A PILOT STUDY BASED ON THE EUROPEAN CENTRE FOR DISEASE PREVENTION AND CONTROL (ECDC) INDICATORS

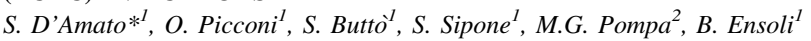
${ }^{I}$ National AIDS Center, Istituto Superiore di Sanità, Rome, Italy; ${ }^{2}$ Direzione Generale della Prevenzione SanitariaSanitaria, Ministero della Salute, Rome, Italy

Background: Virus transmission via heterosexual intercourses among migrants represents about $40 \%$ out of all new HIV infections due to sexual intercourses in Europe. Italian data, provided by the AIDS Operational Centre of the Italian National Institute of Health, confirm an increase of new HIV infection diagnosis among migrants, raised from $11 \%$ in 1992 to $31.6 \%$ in 2008 ; heterosexual intercourses among foreign people are the most common mode of transmission $(55.2 \%)$ and in particular the proportion of migrants who have been infected through sexual intercourses passes from $24.6 \%$ in 1992 to $64.3 \%$ in 2008. Another key point, provided by the COA, is the increase in the proportion of foreigners among TB and AIDS co-infection cases, which passed from $10.8 \%$ in 1993 to $76.9 \%$ in 2009. Socio-economic conditions, different cultural approaches to infectious diseases and major difficulties in accessing appropriate treatments, are risk factors for isolation among HIV positive migrants. The European guidelines for surveillance and prevention of the most vulnerable groups, suggest to conduct a pilot study to get the following data among migrants: knowledge of HIV/AIDS, STD and TB, \% of access to HIV test with respect to gender, race, age, education, how long they have been staying in Italy, social integration, how the test has been proposed, access to prevention programs and information about HIV/STD/TB, behaviors adopted to prevent infection (in particular use of condom), HIV prevalence, proportion of recent infections among the new HIV diagnosis, evaluation of circulating HIV subtypes. This project, in collaboration with the Associations of the Council for the fight against AIDS, aims to promote and offer the HIV testing among migrants.

Methods: General objective is to monitor some ECDC parameters for HIV prevention and to estimate some immunological and virological parameters among migrants, who refer to five clinical centers for the HIV infection diagnosis (Bari, Brescia, Florence, Palermo, Rome), in order to provide data on HIV infection. Specific objectives are the assessment of: percentage of access to the HIV testing; access to prevention programs; access to correct information about HIV; percentage of condom use; proportion of recent infections; evaluation of circulating HIV subtypes;

Results: Monitoring: HIV prevalence estimate; number of new infections difference of circulating HIV; percentage of access to prevention programs and correct information about HIV; data about information needs among the population target; data about HIV infection management among migrants, through social and health institutions.

Conclusions: The results will be helpful for Associations involved in the field, cultural mediators, health workers, to assess innovative and targeted prevention and information strategies for the population target and to favor the early settling in of foreign people in the care pathways for the cure of the HIV infection.

\section{CO 12}

\section{HIV POST-EXPOSURE PROPHYLAXIS IN ITALY (1996-2009)}

V. Puro* ${ }^{l}$, G. De Carli ${ }^{1}$, E. Schifano ${ }^{1}$, P. Piselli ${ }^{l}$, N. Orchi ${ }^{1}$, S. Pittalis ${ }^{I}$,

P. Scognamiglio ${ }^{I}$, C. Cimaglia ${ }^{I}, A$. Agresta ${ }^{I}$, G. Ippolito ${ }^{I}$, Italian Registry of Antireroviral PEP

${ }^{I}$ INMI "L. Spallanzani" IRCCS, Rome, Italy

Background: Post-exposure prophylaxis (PEP) is widely used after exposures to HIV. PEP-associated side effects (SE) often occur, causing discontinuation.

Methods: We reviewed data collected in the Italian PEP Registry (1996-2009). Cases were analyzed according to gender, initial regimen, and reasons for PPE discontinuation (D). Outcomes were development of at least one SE and D ( $<28$ days), as well as followup at 90 and 180 days.

Results: As of 2009 the Registry included 4,335 cases $(52.8 \%$ women; median age 34 years, range 3-82): 2,633 healthcare workers (HCW), 326 occupationally exposed safety/social workers (SSW), 918 persons exposed by sexual route (ESR) and 458 with other exposures (OE); 61 persons have performed multiple PEP (2 SSW, 56 ESR, $3 \mathrm{OE})$. Source patients resulted to be negative in 1,132 (26\% of 
all cases) and PPE was discontinued. Ten exposed subjects tested HIV-positive at baseline. On 3,193 HIV-negative cases (73.7\%) with HIV-positive or unknown source, for whom PPE was provided, 1,632 were $\mathrm{HCW}, 267 \mathrm{SSW}, 890 \mathrm{ESR}$ and 404 OE. 2,508 (78.6\%) received as initial PPE 2 NRTI + PI, mostly AZT + 3TC + IDV (40\%), NFV (18\%) and LPV (31\%). PPE was initiated within $72 \mathrm{~h}$ in $98 \%$ cases and within $48 \mathrm{~h}$ in $93 \%$ (96\% in HCW, 95\% in SSW, 86\% in ESR and $93 \%$ in OE). 1,952 (61\%) were exposed to a HIV-positive source (620 with detectable and 418 undetectable viraemia, in 914 viremia was unavailable). PEP was discontinued in 988 (31\%) cases, mainly due to SE $(48 \%)$. Overall PEP was completed in $69 \%: 73.7 \%$ in ESR, $68.3 \%$ in HCW, $67.6 \%$ in OE and 60.3 in SSW. PEP-D was associated overall with side effects $(35.7 \%$ of those with vs. $25.9 \%$ of those without SE, $\mathrm{p}<0.001$ ); this was not the case for ESR where no difference in PEP-D was evident (25.4 vs. $27.2 \%, \mathrm{p}=0.559)$. PEP-D was lower in cases with HIV-positive source (25.3 vs. $39.8 \%$ for HIVunknown source). In 2,373 (74.3\%) cases FUP was available: completed (> 90 days) in $62.4 \%$ and further extended to 180 days in $46 \%$. Overall, during follow-up four seroconversions were observed, all in homosexual men belonging to the ESR group, in which at least in three out of four cases an unprotected sexual exposure following or preceding PEP was documented.

Conclusions: A significantly higher risk of D was associated with developing SE, and with occupational exposures among HCWs and SSW in comparison to ESR. The different proportion of PEP-D among groups, the cases of infection at baseline, and the seroconversions observed only in the ESR group, strengthen the need to improve the efficacy of post-exposure counseling and suggest the need for testing different modalities of PEP provision. Other Italian Registry of Antireroviral PEP members: L. Signorini, F. Niero, M. Fantoni, A. Franco, R. Bertucci, A. Vivarelli, G. Morigi, G. Raineri, G. Micheloni, M. Moretti, I. Crosato, G. Fasulo, P. Castelli, C.Calzetti, D. Drenaggi, G. Di Giacomo, S. Laganà, M. Massari, G. Cassola, F. Dodi, B. De Rienzo, C. Penna, C. Martinelli, M. Perosino.

\section{CO 13}

\section{TLR4 RESPONSIVENESS TO LPS STIMULATION IS} UPREGULATED IN HIV-EXPOSED UNINFECTED INDIVIDUALS

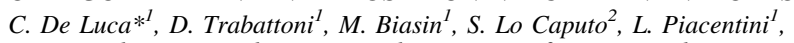
B. Tavano ${ }^{l}$, M. Borelli ${ }^{1}$, V. Rainone ${ }^{I}$, F. Mazzotta ${ }^{2}$, M. Clerici $^{1}$

${ }^{1}$ Università degli Studi di Milano, Milan, Italy; ${ }^{2}$ Osp. S Maria Annunziata, Florence, Italy

Background: Immune activation, the presumed principal reason for the immune deficiency that characterizes HIV infection, is suggested to be the result of the severe damages that affect the gastrointestinal mucosa in the earliest phases of the disease. Several reports indicates that the resulting mucosal alterations allow gut bacterial translocation into the peripheral blood, resulting in Toll-like receptor 4 (TLR4)mediated immune responses.

Materials and methods: PBMCs isolated from 40 HIV-Exposed Uninfected Individuals (ESN) and from 40 healthy controls (HC) were stimulated with agonists specific for TLR4 (LPS). We evaluated expression of factors involved in TLR4 signaling cascades, production of downstream effector immune mediators, TLR4-expression in CD14+ cells, activated T lymphocytes (CD8/CD38/CD45RO, CD4/ CD25) and plasma LPS concentrations.

Results: In comparison with HC, ESN showed: (1) higher responsiveness to LPS stimulation, associated with significantly increased production of IL-1beta, IL-6, TNF-alpha, IL-8, IL-10, CCL3, CSF3 and $\mathrm{COX} 2$, as demonstrated by TLRs pathway expression analyses; (2) comparable percentages of CD14/TLR4 cells; (3) increased percentage of CD8/CD38/CD45RO, CD4/CD25 T cells; and (4) similar plasma LPS levels.
Conclusions: In ESN individuals intestinal barrier functions are maintained despite hyperactivation of peripheral immune responses. These data suggest that in the absence of alterations in gut permeability, strong adaptive antiviral immune responses and immune activation correlate with resistance to HIV infection.

\section{CO 14}

IN VITRO EXPOSURE OF HEALTHY CD4+ T LYMPHOCYTES TO GP120 INDUCES CHANGES IN MIRNA EXPRESSION PROFILES C. Casoli $*^{1,2}$

${ }^{1}$ Università di Milano, Dipartimento di Scienze Cliniche, sez. Malattie Infettive, Milan, Italy; ${ }^{2}$ Gemib srl, Parma, Italy

Background: miRNAs inhibit viral expression by either modulating host innate immunity or by directly interfering with viral mRNAs. However cellular miRNA expression may also favor virus infection. Previously, we demonstrated that miRNA expression may discriminate between HIV-1 infected and exposed but negative individuals. These findings suggested that miRNA profile could be the result not only of a productive infection, but also of the exposure to HIV products that leave a signature in immune cells. Here, we investigated the miRNA expression profile in CD4+ $\mathrm{T}$ cells after exposure to gp120 or after in vitro infection by HIV isolates.

Methods: CD4+ cells were isolated from a pool of six healthy subjects. The expression levels of 377 miRNAs were selectively analyzed in CD4+ $\mathrm{T}$ cells exposed to gp120 (recombinant or natural proteins) or infected in vitro with HIV strains. MiRNAs extraction was performed by the mirVana miRNA Isolation Kit (Ambion) and their expression was subsequently examined by real-time PCR-based arrays. Significant differences in expression of miRNAs were evaluated using a linear mixed effects model of two-way analysis of variance (ANOVA) followed by Bonferroni post hoc tests.

Results: Exposure to recombinant and natural gp120 molecules induced the modulation of 43 and 51 miRNAs, respectively. Expression levels of 28 miRNAs were altered by both molecules. The remaining miRNAs showed a non comparable expression presumably due to different molecular structure of the two tested gp120. When the gp120 action was neutralized with specific mAbs, about $1 / 3$ of altered miRNAs were reverted to the expression of controls. Presumably, the expression of these reverted miRNAs could be altered by gp120 through the CD4-mediated signaling. Five reverted miRNAs (34c-5p, 518f, 452, 202, 487b), which expression may be related to CD4/gp120 binding site, were also found altered at the same expression level after in vitro infection of CD4+ lymphocytes.

Conclusions: These data support the hypothesis that the exposure to HIV antigen (e.g., gp120) can be enough to induce a marked alteration of miRNA patterns and consequently may influence immune function of $\mathrm{CD} 4+\mathrm{T}$ cells. These data provide some intriguing issues relative to the development of HIV vaccines targeting viral proteins.

\section{CO 16}

COMPARATIVE PROTEOMIC ANALYSIS OF DLBCL FROM HIV+ AND HIV - INDIVIDUALS BY 2D-DIFFERENTIAL GEL ELECTROPHORESIS

V. De Re ${ }^{* 1}$, M.P. Simula ${ }^{I}$, A. Pavan ${ }^{I}$, G. Toffoli ${ }^{I}$, A. Carbone ${ }^{I}$, V. Canzonieri ${ }^{I}$, M. Spinal, E. Vaccher ${ }^{1}$

${ }^{I}$ Centro di Riferimento Oncologico, Aviano, Italy

Background: Diffuse large B-cell lymphomas (DLBCL) are associated with different morpho-phenotypes and clinical outcome. Despite treatments (CHOP for GC subtype and R-CHOP for both GC and ABC-like subtypes), several patients remain unresponsive and/or experience relapse(s). In HIV+ patients, the role of immunosuppression induced by the virus and the efficacy of antiviral treatment in 
tumor response is still unclear. The aim of the study was to investigate whether an integrated proteomic and immunohistochemical approach may discriminate proteins expression profile between HIV+ and HIV- DLBCL patients with good clinical response upon R-CHOP.

Methods: Two dimensional fluorescence difference gel electrophoresis (2D-DIGE) was used to identify proteins that are differentially expressed in affected lymph nodes from eight patients (5 HIV - and 3 HIV+) with DLBCL experiencing a good clinical response upon $\mathrm{R}-\mathrm{CHOP}$ at 5 years follow-up. Immunohistochemistry was applied for analysis of selected proteins in tumor tissues.

Results: Data analysis by 2D-DIGE showed a limited number $(23 / 250$ analyzed, $<10 \%)$ of differentially expressed proteins $(\mathrm{p}=0.05)$ in HIV+ versus HIV - DLBCL patients. Difference spans ranged from a maximum of 3.53 times (anti-trypsin) to a minimum of -2.91 times (serpin). Proteins were clustered as cytoskeletric, extracellular matrix proteins, metabolic enzymes and proteins involved in the unfolding endoplasmatic process. Immunohistochemical analyses confirmed the high expression of proteins associated with histiocytes/macrophagic population.

Conclusion We found a similar protein expression profile in HIV+ versus HIV - DLBCL patients. For several proteins there was good agreement between proteomic and immunohistochemical findings, providing insights into the biomolecular mechanisms involved in these malignancies and their possible use as biomarkers for good clinical response.

\section{CO 17}

IL-7 RECEPTOR AND IL-15 RECEPTOR SINGLE NUCLEOTIDE POLYMORPHISMS INFLUENCE HIV-1 DISEASE PROGRESSION A. Riva ${ }^{*}$, L. Ferraris ${ }^{2}$, D. Misciagna ${ }^{2}$, M. Nasi ${ }^{3}$, S. Ghezzi ${ }^{4}$, C. Casoli ${ }^{2}$, G. Poli ${ }^{5}$, A. Cossarizza ${ }^{3}, M$. Galli ${ }^{2}, M$. Tarkowski ${ }^{2}$

${ }^{1}$ III Divisione di Malattie Infettive, Ospedale L. Sacco, Milan, Italy; ${ }^{2}$ Dipartimento di Scienze Cliniche, Sezione da Malattie Infettive e

Immunopatogenesi, Università di Milano, Milan, Italy, ${ }^{3}$ Dipartimento di Scieze Biomediche, Università di Modena e Reggio Emilia, Modena, Italy; ${ }^{4}$ Unità di Immunopatogenesi dell'AIDS, Divisione di Immunologia, Università VitaSalute, San Raffaele, Milan, Italy; ${ }^{5}$ Unità di Immunopatogenesi dell'AIDS, Divisione di Immunologia, Università Vita e Salute, San Raffaele, Milan, Italy

Aims: Both interleukin-7 and interleukin-15 are necessary for CD4 and CD8 T cells homeostasis. Single nucleotide polymorphisms (SNPs) may be involved in the rate of HIV disease progression. The aim of this study was to evaluate the role of polymorphisms located in IL-7 and IL-15 and their cognate receptor genes in HIV disease progression.

Methods: We genotyped 91 antiretroviral treated patients (progressors) and 121 long term non progressors (LTNP) using a real time PCR methodology. We analyzed five polymorphisms in IL-7R, one in IL-7, three in IL-15R and two in IL-15 genes. We evaluated cell surface receptor expression by means of cytometry.

Results: In univariate analysis, we found an association between the presence of at least one mutated allele $A$ in the IL-15R SNP rs2228059 and a higher possibility of being LTNP (AOR 2.08, 95\% CI $1.13-3.85$ vs. CC, $\mathrm{p}=0.019$ ); as well as the presence of at least one mutated allele $\mathrm{G}$ and lack of disease progression (AOR 2.29, $95 \%$ CI $1.34-3.94$ vs. $\mathrm{CC}, \mathrm{p}=0.003$ ). In a multivariate model the presence of at least one mutated allele G in IL-7R SNP rs3194051 or A in IL-15R rs2228059 was associated to the condition of non progression (OR 2.31, 95\% CI 1.33-3.99 vs. CC, $\mathrm{p}=0.003$ and OR $2.08,95 \%$ CI $1.11-3.69$ vs. $\mathrm{CC}, \mathrm{p}=0.022$, respectively). The co-presence of two favourable alleles in IL-15R and in IL-7R determined a higher association with non progression (OR 2.68, 95\% CI $1.47-4.52$ vs. allele $\mathrm{C}+$ allele $\mathrm{G}, \mathrm{p}=0.001)$. We therefore analyzed IL-15R expression on peripheral blood mononuclear cells according to the genotype of SNP rs2228059 and found a significantly increased expression from wild type to heterozygous to mutated homozygous. Other SNP were not linked to disease progression.

Conclusions: Our study suggests that genetic polymorphisms located in IL-7R and IL-15R genes can influence the rate of disease progression in HIV+ patients, especially when a combination of aplotypes is present. SNP rs2228059 is able to influence IL-15 receptor expression. Taking into consideration the role of IL-15 as a growth factor for T cells and NK cells and the conserved functionality of CD8 T cells in LTNP, we speculate that this IL-15R SNP could contribute to long lasting protection from HIV disease progression through increased IL-15 receptor expression determining higher activity of cytotoxic CD8 T cells and/or NK cells.

CO 21

EXPANSION OF ACTIVATED T LYMPHOCYTES

(CD3+CD4+HLADR+ AND CD3+CD8+HLADR+) IN PATIENTS TREATED WITH HAART WITH VIRAL BLIPS OR LOW LEVELS OF RESIDUAL VIREMIA

A.R. Buonomini ${ }^{\prime}{ }^{1}$, L. Sarmati ${ }^{1}$, M. Viscione $^{1}$, L. Dori ${ }^{1}$, G. Maffongelli ${ }^{1}$ D. Fraboni ${ }^{I}$, F. Buccisano ${ }^{I}$, S.G. Parisi ${ }^{3}$, R. Ferretto ${ }^{2}$, M. Andreoni ${ }^{l}$ ${ }^{1}$ Cattedra di Malattie Infettive, Tor Vergata, Rome, Italy; ${ }^{2}$ Ospedale di Schio, Vicenza, Italy; ${ }^{3}$ Dipartimento di istologia, microbiologia e biotecnologie, Università di Padova, Padua, Italy

Background: Increased levels of activated T cells are a hallmark of the chronic stage of human immunodeficiency virus (HIV) infection and are highly correlated with HIV disease progression. The clinical significance of persistent residual viremia in patients on prolonged highly active antiretroviral therapy (HAART) is not clear and the effect of low-level viremia on T-cell homeostasis is still debated. This study was designed to investigate the correlation between markers of immune-activation and low levels of HIV viremia in HAART treated patients.

Methods: Twenty-four patients responder to HAART were enrolled in the study. A strict monitoring of HIV viremia was conducted during a 2 year period. Twelve patients resulted persistently HIVRNA undetectable (less of $50 \mathrm{cp} / \mathrm{ml}$ ) and 12 presented transient lowdetectable viremia ('blips') (below $1,000 \mathrm{cp} / \mathrm{ml}$ ). The mean time of treatment was 29 and 23 months, respectively, for the two groups with a median in CD4 gain of 409 and 364 cells $/ \mathrm{ml}$, respectively. Evaluation of lymphocyte phenotype was performed by flow cytometric and multiparameter analysis on total lymphocytes, $\mathrm{T}$ helper and suppressor lymphocytes (naive and memory), B cells, NK cells and regulatory $\mathrm{T}$ cells $(\mathrm{CD} 4+\mathrm{CD} 25-\mathrm{CD} 127)$.

Results: No differences in demographic parameters were found between the two groups. In patients with viral blips the number of HLA-DR+CD3 lymphocytes were higher $(\mathrm{p}=0.03)$ than in patients with persistent undetectable HIV viremia. Both CD4+ and CD8+ lymphocytes expressed HLA-DR antigens. Patients with HIV viremia persistently under $50 \mathrm{cp} / \mathrm{ml}$ had a significant $(\mathrm{p}=0.04)$ higher percentage of $\mathrm{CD} 4+$ compared to patients with viral blips. Analyzing the subgroup of 12 patients with a stable undetectable viremia, significant higher number of HLA-DR+CD3 $(\mathrm{p}=0.003)$ was present in 6 patients with $>30 \mathrm{cp} / \mathrm{ml}$ than in those with $<30 \mathrm{cp} / \mathrm{ml}$. No differences were found among the different groups of subjects in regard to the expression of CD25 and CD127.

Conclusion In HAART treated patients with occasional viral blips or with ultrasensitive viremia $>30 \mathrm{cp} / \mathrm{ml}$ a significant higher numbers of activated $\mathrm{CD} 3+$ lymphocytes have been demonstrated compared to patients with low level of residual viremia $(<30 \mathrm{cp} / \mathrm{ml})$. These results suggest that low-level HIV-1 viremia may contribute to persistent $\mathrm{T}$-cell activation and to alter $\mathrm{T}$ cell immune response despite HAART. 
CO 22

IMMUNOLOGICAL EFFICACY OF MARAVIROC (MVC) AS INTENSIFICATION STRATEGY IN HIV-INFECTED PATIENTS (PTS) FAILING CD4 RECOVERY ON VIROLOGICALLY-SUPPRESSIVE HAART

S. Rusconi ${ }^{1}$, E. Colella ${ }^{1}$, F. Adorni ${ }^{2}$, P. Vitiello ${ }^{1}$, E. Foca ${ }^{3}$, A. Capetti ${ }^{4}$, P. Meraviglia ${ }^{5}$, C. Abeli ${ }^{6}$, S. Bonora ${ }^{7}$, M. D'Annunzio $^{8}$, A. Di Biagio ${ }^{9}$, M. Di Pietro ${ }^{10}$, L. Butini ${ }^{11}$, G. Orofino ${ }^{12}$, S. Farina ${ }^{13}$, G. D'Ettorre ${ }^{14}$, D. Francisci ${ }^{15}$, A. Soria ${ }^{16}$, G. Parruti ${ }^{17}$, A.R. Buonomini ${ }^{18}$, C. Tommasi ${ }^{19}$, M.P. Trotta ${ }^{20}$, E. Merlini ${ }^{21}$, G. Marchetti ${ }^{21}$

${ }^{I}$ Dipartimento di Scienze Cliniche "Luigi Sacco", Sezione di Malattie Infettive e Immunopatologia, Università degli Studi di Milano, Milan, Italy; ${ }^{2}$ ITB-CNR, Segrate, MI, Italy; ${ }^{3}$ Clinica Malattie Infettive, Università degli Studi di Brescia, Spedali Civili, Brescia, Italy; ${ }^{4}$ la Div. Malattie Infettive, Ospedale Luigi Sacco, Milan, Italy; ${ }^{5}$ a Div. Malattie Infettive, Ospedale Luigi Sacco, Milan, Italy; ${ }^{6}$ Div. Malattie Infettive, Ospedale di Circolo, Busto Arsizio, VA, Italy; ${ }^{7}$ Clinica Malattie Infettive, Università degli Studi di Torino, Turin, Italy; ${ }^{8}$ Clinica di Malattie Infettive, Ospedale Policlinico, Bari, Italy; ${ }^{9}$ Clinica Malattie Infettive, Università degli Studi di Genova, Ospedale San Martino, Genoa, Italy; ${ }^{10}$ Div. Malattie Infettive, Ospedale S. Maria Annunziata, Antella, Florence, Italy; ${ }^{11}$ Servizio Regionale di Immunologia Clinica e Tipizzazione Tessutale, Università Politecnica delle Marche, Torrette di Ancona, AN, Italy; ${ }^{2}$ Div. A Malattie Infettive, Ospedale Amedeo di Savoia, Torino; ${ }^{13}$ Istituto di Clinica Malattie Infettive, Università Cattolica del Sacro Cuore, Rome, Italy; ${ }^{14}$ Div. Malattie Infettive, Ospedale Policlinico Umberto I, Rome, Italy; ${ }^{15}$ Clinica di Malattie Infettive, Ospedale S. Maria della Misericordia, Perugia, Italy; ${ }^{16}$ Div. Malattie Infettive, Ospedale San Gerardo, Monza, Italy; ${ }^{17}$ Div. Malattie Infettive, Ospedale Santo Spirito, Pescara, Italy; ${ }^{18}$ Clinica Malattie Infettive, Università Tor Vergata, Rome, Italy; ${ }^{19}$ INMI "Lazzaro Spallanzani", IV Div. Malattie Infettive, Rome, Italy; ${ }^{20}$ INMI "Lazzaro Spallanzani", III Div. Malattie Infettive, Rome, Italy; ${ }^{21}$ Clinica Malattie Infettive e Tropicali, Università degli Studi di Milano, Ospedale San Paolo, Milan, Italy

Background: HIV-infected pts failing immune recovery on virologically-suppressive HAART maintain a highly activated/ differentiated peripheral CD4 pool. We hypothesized that MVC, by specifically targeting terminally-differentiated CCR5+CD4, might preserve the naive/central memory CD4 pool in these pts.

Methods: Pts on HAART with CD $4<200 / \mu \mathrm{L}$ and HIV-RNA $<50 \mathrm{cp} /$ $\mathrm{mL}$ were randomized to: HAART+MVC (A) or continuing HAART (B). Naïve CD45RA+62L+, memory CD45RA-, central-memory CD127+, activated/proliferating HLA-DR+CD38+Ki67+CD4/CD8, plasma IL-7 were quantified. HIV-RNA was quantified via Amplicor HIV-1 Monitor Kit v1.5, followed by RT-PCR.

Results: 66/100 pts were analyzed at week 12 (W12): 39 in A; 27 in B. At baseline, comparable total CD4 and immune-phenotypes were shown between arms. By W12, both study groups displayed a significant increase in CD4 (A: 184-230, p <.001; B: 165-190, $\mathrm{p}=.037)$. A statistically significant change in mean CD8 $(\mathrm{p}=.002)$ was observed between pts in arm A and B. HIV-RNA remained $<50 \mathrm{cp} / \mathrm{mL}$ with no difference between arms. The immunephenotype of reconstituting CD4 was different between arms. Whereas HAARTcontrols displayed a rise in memory CD4 $(53-70 \%, \mathrm{p}=.05)$ with a contraction of naive T-cells (CD4, 24-9\%, p =.02; CD8, 20-9\%, $\mathrm{p}=.028)$, MVC-receiving patients maintained stable memory T-cells (CD4, 35-45\%, $\mathrm{p}=.14$; CD8, 35-38\%, $\mathrm{p}=.08$ ) with a non-significant rise in naive CD4 $(17-37 \%, \mathrm{p}=.3)$ and $\operatorname{CD} 8(11-36 \%, \mathrm{p}=.3)$. Interestingly, MCV resulted in a significantly higher proportion of naïve CD4 compared to controls $(\mathrm{p}=.03)$, whereas controls tended to have higher memory CD4 $(\mathrm{p}=.08)$. Both MVC and HAART resulted in the decline of activated HLA-DR+CD38+ T-cells (A: $\mathrm{p}=.03, \mathrm{p}=.03 ; \mathrm{B}: \mathrm{p}=.009, \mathrm{p}=.038$ for $\mathrm{CD} 4$ and $\mathrm{CD} 8$, respectively). Despite no changes in Ki67+CD4/CD8 in both groups, MVCreceiving pts displayed significantly lower proliferating Ki67+CD4 at W12 (18 vs. $23 \% \mathrm{p}=.04$ ). As for the IL-7/IL-7R, only MVCreceiving patients presented an increasing trend in central-memory
CD127+CD4 by W12 $(56-60 \%, \mathrm{p}=.058)$, with no change in CD127 + CD8 and circulating IL-7.

Conclusions: In HIV-infected subjects with inefficient immune reconstitution on virologically-suppressive HAART, intensification with MVC results in a significant expansion of naïve CD4 pool that proliferates less actively. This suggests a reduction in peripheral T-cell death and preserved T-cell production, with possible improvement in immune competence.

\section{CO 23}

FIRST DEMONSTRATION OF A DIRECT VIRAL DAMAGE ON HUMAN VESSEL WALL DERIVED MESENCHYMAL STEM CELLS BIOLOGIC HOMEOSTASIS: A NEW ROLE OF HIV-1 INFECTION AND RECOMBINANT GP120 IN THE GENESIS OF ATHEROSCLEROTIC DEGENERATION AND ADIPOGENESIS INDUCTION

M. Borderi ${ }^{I}$, D. Gibellini ${ }^{2}$, V. Colangeli ${ }^{1}$, L. Calza ${ }^{1}$, R. Manfredi ${ }^{1}$, C. Marsiglia ${ }^{3}$, G. Taliani ${ }^{3}$, M.C. Re ${ }^{2}$, P. Viale ${ }^{1}$

${ }^{I}$ U.O. Malattie Infettive, Bologna, Italy; ${ }^{2}$ Istituto di Microbiologia, Bologna, Italy; ${ }^{3}$ Clinica Malattie Infettive e Tropicali, Università La Sapienza, Rome, Italy

Background: Pluripotent MSCs were isolated in the adventitia and in subendothelial region of vessels and can be differentiated towards several cell lineages such as endothelial cells, osteoblasts, adipocytes and smooth muscle cells. Hence, these cells may be a target of HIV and/or viral proteins inducing direct or indirect induction of vessel damages. We have focused our attention on two main aspects represented by the direct effects of HIV-1 challenge and gp120 treatment on primary vessel wall MSCs and the impact of viral action on MSC differentiation towards specific lineages.

Materials and methods: Human arterial segments of femoral arteries from three male heart-beating donors were harvested, processed for immunohistochemical examination and used for cell isolation. The characterization of isolated cells, as mesenchymal stem cells (MSCs), was carried out through flow cytometry procedure and multi-differentiation potential analysis. The apoptotic cells were analyzed on primary sub-confluent MSCs challenged with HIV-1 strains, hi HIV-1 strains or gp120. PPARg transcription factor activity was detected by TransAM PPARg kit. The data are expressed as mean + standard deviation (SD) of three separate experiments performed in duplicate. Statistical analysis was performed using two-tailed Student's $t$ test.

Results: R5 and X4 HIV-1 laboratory strains were challenged with MSCs determining that these strains are able to entry and integrate their retrotranscribed proviral DNA in the host cell genome. HIV-1 strains, recombinant gp120 (rgp120) and heat-inactivated HIV-1 strains elicited a reliable increase of apoptosis in subconfluent MSCs through the interaction between rgp120 and cell membrane. We have challenged HIV-1 strains, rgp120 and heat-inactivated HIV-1 strains on MSCs differentiated to adipogenesis and endotheliogenesis displaying that the adipogenesis is increased especially through the PPARg activity up-regulation whereas the endothelial differentiation, induced by VEGF treatment, was clearly impaired by the same treatment with a down-regulation of endothelial markers such as vWF, VEGF-R1 and R2 expression.

Discussion: Our study indicate that HIV and recombinant gp120 have a strong direct impact on vessel wall MSC biology and differentiation suggesting a role of viral infection in the vessel damage, atherosclerosis degeneration and adipogenesis induction. Our data suggested an additional mechanism in the damage of endothelial layer involving the MSC role in the vessel homeostasis. HIV affects the MSC biology acting both on primary MSCs and MSCs differentiated to adipocyte and endothelial cells. In addition, the impairment of MSC differentiation is noteworthy because in the atherosclerotic vessel degeneration it is possible remark some peculiar lesions such as cartilaginous metaplasia with endocondral ossification, fat tissues 
especially in inflammatory abdominal aortic aneurysms, that might be related to MSC differentiation derangement.

\section{CO 25}

HAART, LOW LEVEL RESIDUAL VIREMIA AND RISK

OF VIROLOGICAL FAILURE.

G. Cologni ${ }^{\prime}$, C. Bernardini ${ }^{I}$, A. Callegaro ${ }^{1}$, L. Soavi ${ }^{I}$, S. Leone ${ }^{I}$, D. Valenti ${ }^{I}$, F. Suter ${ }^{1}$, F. Maggiolo ${ }^{1}$

${ }^{I}$ Ospedali Riuniti, Bergamo, Italy

Purpose: The clinical relevance of low level residual viremia (LLV) in patients on steady HAART is debated. Similarly the clinical usefulness of HIV-RNA cut-offs lower than 50 copies $/ \mathrm{ml}$ is questioned. Aim of this study was to analyse the dynamics of LLV in patients on HAART by means of a high resolution test for HIV-RNA.

Methods: This is a prospective, single-center, cohort study in patients on stable HAART (mean time on HAART 106 months, SD 40). All patients with a confirmed viremia $<50$ copies $/ \mathrm{ml}$ were enrolled. Patients were monitored prospectively every 4 months with an enhanced PCR test with a lower limit of detection of 3 copies $/ \mathrm{ml}$. ITT analysis is reported.

Results: 1,042 patients were screened; 38 were excluded from the analysis because of incomplete data. 1,004 patients ( $76 \%$ males) with a mean age of 46.2 years (SD 8) were enrolled. At baseline the mean CD4 count was 632 cells/mcL (SD 269) and VL was $<3$ copies $/ \mathrm{ml}$ in $73.7 \%$ and between 3 and 50 copies $/ \mathrm{ml}$ in $26.3 \%$ of cases. Over the following 8 months period, patients with a baseline VL $<3$ copies presented a stable HIV-RNA below this threshold in $67.2 \%$ of cases, a level between 3 and 50 copies $/ \mathrm{ml}$ in $30.0 \%$ of cases and at least a viral blip $>50$ copies $/ \mathrm{ml}$ in $2.8 \%$. The same values for patients with a baseline HIV-RNA between 3 and 50 copies $/ \mathrm{ml}$ were $34.8,53.4$ and $11.7 \%$, respectively $(\mathrm{P}<0.0001)$. There was a linear relationship between baseline VL and the risk of viral blips over 50 copies $/ \mathrm{ml}$ : $2.8 \%$ for baseline VL $<3 ; 6.6$ (VL 3-9); 11.7\% (VL 10-20) and $20.3 \%$ (VL $>20)(\mathrm{P}<0.0001)$. Receiver operating curve analysis confirmed the result (ROC AUC 0.695; 95\% CI 0.613-0.778; $\mathrm{P}<0.0001$ ). In a multivariate model the only variable significantly associated with viral dynamics was the third drug in the HAART regimen $(\mathrm{P}=0.009)$. A steady $\mathrm{VL}<3$ copies $/ \mathrm{ml}$, a VL between 3 and $50 \mathrm{copies} / \mathrm{ml}$ or at least a measure $>50 \mathrm{copies} / \mathrm{ml}$ was detected, respectively, in $63.4 ; 34.4$ and $2.2 \%$ of NNRTI-treated patients, while the same figures for PI-treated subjects were 52.4, 39.8 and $8.8 \%$ $(\mathrm{P}<0.0001)$.

Conclusions: The presence of a LLV is associated to a low risk of virological failure, however, in selected patients it may be indicative of effective virus replication leading to virological rebound. Patients treated with a NNRTI-based HAART compared to those receiving a PI-based regimen show a statistically significant more pronounced and steady control of viral replication.

\section{CO 26}

USEFULNESS OF QUANTITATIVE ULTRASONOGRAPHY (QUS) FOR BONE HEALTH ASSESSMENT IN HIV-INFECTED PATIENTS

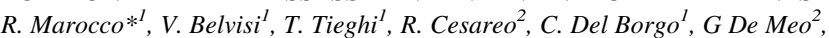
V.S. Mercurio ${ }^{I}$, M. Lichtner ${ }^{I}$, C.M. Mastroianni ${ }^{I}$

${ }^{I}$ Infectious Disease Unit, Sapienza University, SM Goretti Hospital, Latina, Italy; ${ }^{2}$ SM Goretti Hospital Latina, Latina, Italy

Background: In recent years, the importance of bone disease as noninfectious comorbidity involving younger and older HIV-infected individuals has increased. The development of reliable and low cost methods to identify and monitor bone alterations in HIV+ patients is an area of active investigation. Recent data in normal population suggest that bone mineral quality (BMQ), assessed by a quantitative ultrasonography (QUS) could be an early marker of osteopenia/ osteoporosis.
Objective: To investigate bone health assessment in HIV-infected adults using QUS technique and dual energy X-ray absorptiometry (DEXA) and, in addition, to compare the data with viro-immunologic parameters and vitamin D levels.

Methods: 37 HIV-infected patients and 44 HIV-negative controls, matched for sex and age, were enrolled. Bone health was measured using classical dual energy X-ray absorptiometry (DEXA) of spine and hip and calcaneal QUS. Broadband ultrasound attenuation (BUA) and speed of sound (SOS) and quantitative ultrasound index (QUI)/ stiffness index (SI) were assessed by QUS. Data were correlated with CD4+ count, HIV load, years of disease, immune activation markers (DR+CD38+CD4+ and DR+CD38+CD8+), 25OH vitamin D. Nonparametric Mann-Whitney test and Spearman correlation were used for statistical analysis.

Results: 5 patients were viremic (ARV-naïve) and 32 were virologically ARV-suppressed. 17 patients were treated with PI-based regimen and 16 with NNRTI regimen. A significant decrease of QUI/ SI parameters was found in HIV-infected patients in comparison to control $(83.4 \pm 4.9 ; 99.7 \pm 2.5, \mathrm{p}=0.014)$. An inverse correlation between QUI and age in HIV+ patients was seen $(r=-0.38$; $\mathrm{p}=0.047$ ). In particular, the highest difference in QUI parameters was found in HIV+ patients aged $>48$ years $(p=0.012)$. Moreover, a strong correlation between QUI and DEXA values was observed $(p=0.001)$. Comparable QUS parameters were found between NNRTI- and PI-based therapies. No difference was seen in patients treated with TDF. No difference was observed between HIV+ subjects and controls for vitamin D levels which were decreased in both groups. In HIV+ people, we found a significant correlation between vitamin $\mathrm{D}$ level and age $(\mathrm{p}=0.022)$, and between vitamin $\mathrm{D}$ and years of infection $(p=0.0142)$. No correlation was found between QUS and CD4 nadir, as well as between QUS and immune activation markers.

Conclusions: QUS has been introduced in the medical field for the study of bone tissue in normal population to identify changes in the tissue that could suggest the presence of osteoporosis and bone fragility. Our data in HIV-infected people suggest that the measurement of QUS parameters might be an additional, simple and inexpensive technique to monitor bone status and identify early signs of bone damage.

\section{CO 27}

TENOFOVIR AND EMTRICITABINE CSF-TO-PLASMA RATIOS CORRELATE TO THE EXTENT OF BLOOD-BRAIN-BARRIER DAMAGE

A. Calcagno $^{* 1}$, S. Bonora ${ }^{1}$, M. Simiele ${ }^{2}$, S. Audagnotto ${ }^{I}$, M. Siccardi ${ }^{2}$, D. Gonzalez de Requena ${ }^{2}$, A. Romito ${ }^{4}$, D. Imperiale ${ }^{3}$, A. D'Avolio ${ }^{2}$, G. Di Perri ${ }^{I}$

${ }^{I}$ Department of Infectious Diseases, University of Torino, Turin, Italy; ${ }^{2}$ Pharmacokinetics and Pharmacogenetics Laboratory, University of Torino, Turin, Italy; ${ }^{3}$ Department of Neurology, Ospedale Maria Vittoria, ASLTO2, Turin, Italy; ${ }^{4}$ Immunology Laboratory, Ospedale Maria Vittoria, ASLTO2, Turin, Italy

Background: Antiretrovirals (ARVs) penetration and effectiveness in the central nervous system have been shown to be related to the extent of HIV suppression in this sanctuary site. Drug chemical properties as well as blood-brain-barrier (BBB) integrity influence the passage to the cerebrospinal fluid (CSF). Alterations in the BBB are common during the course of HIV infection but few is known on the effect of such modifications on the CSF-to-plasma ratios of ARVs.

Methods: Samples from patients undergoing lumbar punctures for clinical reasons were analyzed for BBB integrity indexes (Albumin, IgG and Tourtelotte); concomitant plasma and CSF concentrations were collected using a sparse sampling strategy and measured with validated HPLC-MS methods. Data are expressed as median (interquartile range); Spearman's analysis were used to test association between variables. 
Results: Eighteen patients on antiretroviral treatment containing Truvada ${ }^{\circledR}$ were included in this analysis. Median age and BMI were 39 years $(35-50)$ and $22 \mathrm{~kg} / \mathrm{m}^{2}(20.4-24.8) ; 44.4 \%$ of them were male. Samples were collected 14-26 h after drug intake; concomitant drugs were mostly boosted protease inhibitors $(69.2 \%)$ and NNRTIs (23\%). BBB integrity was altered in eight $(44.4 \%)$ of the patients with albumin and IgG ratios ranging respectively from 2.4 to 19.5 and 1.8 to 22. Tenofovir and emtricitabine CSF-to-plasma ratios were 0.05 $(0-0.14)$ and $0.37(0-0.48)$; the two ratios were significantly related to each other $(\rho=0.83, \mathrm{p}=0.002)$. Both drug ratios were significantly correlated to the albumin CSF-to-plasma indexes (respectively $\rho=0.57$ with $\mathrm{p}=0.017$ and $\rho=0.57$ with $\mathrm{p}=0.05$ ).

Conclusion: Tenofovir and emtricitabine passage to the CSF were similar to the reported ones and they were apparently related to each other: patients with higher ratios of one drug showed higher ratios of the other one. This observation, though predictable from the chemical properties of the two drugs, could hint for pharmacogenetic differences or alterations in the blood-brain-barrier integrity. The association between these drugs and albumin CSF-to-plasma ratios suggest that disruption in the $\mathrm{BBB}$ could partially explain the observed variability in the cerebrospinal penetration of antiretrovirals.

\section{CO 28}

VALUE OF ANTIRETROVIRAL CNS PENETRATIONEFFECTIVENESS (CPE) 2010 RANKING IN PREDICTING CSF HIV REPLICATION ACCORDING WITH RATE OF VIRAL SUPPRESSION IN PLASMA

G. Picchi $*^{1}$, M.L. Giancola ${ }^{1}$, P. Lorenzini ${ }^{1}$, F. Baldini ${ }^{1}$, L. Monno ${ }^{2}$,

A. Ammassari ${ }^{1}$, A. d'Arminio Monforte ${ }^{3}, P$. Cinque ${ }^{4}$, V. Tozzi ${ }^{1}$, A. Antinori ${ }^{1}$ ${ }^{I}$ INMI L. Spallanzani IRCCS, Rome, Italy; ${ }^{2}$ Università di Bari, Bari, Italy;

${ }^{3}$ A.O. San Paolo, Università di Milano, Milan, Italy; ${ }^{4} H S R$, Isituto San

Raffaele, Milan, Italy

Background: HIV-1 replication in CSF despite viral suppression in plasma has been associated with neurocognitive impairment and neurological symptoms. Higher CPE ranking (2010 version) was related to lower proportion of detectable CSF viral load and to longer time-to-loss-of-viral response in CSF, even if predictive value of CPE ranking according to level of HIV-1 suppression in plasma is still unclear.

Methods: Cross-sectional analysis of consecutive paired CSF/plasma samples from HIV infected patients followed at four clinical centers in Italy. Plasma and CSF lower limit of quantification were defined by standard methods at the time of sample collection (range 50-200 cp/ $\mathrm{mL}$ ). Predictive value of covariates associated with HIV-RNA undetectable in CSF were assessed by multivariate linear regression method.

Results: A total of 301 paired CSF/plasma samples collected from 219 HIV-infected patients treated by cART (years 1999-2009) were included in the analysis. Main characteristics: male $81 \%$; median age 42; heterosexual $37 \%$, MSM $15 \%$, IVDU $38 \%$; previous AIDSdefining event $65 \%$. Median CD4 count, HIV-1 RNA values in CSF and in plasma were 129 cells $/ \mu \mathrm{L}, 2.39 \log _{10} / \mathrm{mL}$ and $2.06 \log _{10} / \mathrm{mL}$, respectively. A neurologic disorder was diagnosed in $56 \%$ of patients. Of the 301 samples, 107 (35\%) had undetectable HIV-1 RNA in plasma, and $155(51 \%)$ undetectable values in CSF. Overall median value of antiretroviral CPE 2010 ranking was 7 (IQR 6-8). By multivariate linear regression analysis, higher CPE 2010 ranking was associated with lower value of HIV-RNA in CSF in patients with concomitant suppressed plasma viremia $(\beta$ coefficient $=-4,927.1$; 95\% CI $-8,389.1-1,465.1 ; p=0.006$ ), but not in patients with HIVRNA detectable in plasma ( $\beta$ coefficient $=2,496 ; 95 \%$ CI -39240 $35.156,9 ; \mathrm{p}=0.91)$. Previous AIDS, latest CD4 count and a diagnosis of neurologic disorder were not associated with CSF viremia. Sensitivity analysis indicated CPE ranking cut-off of six as highly predictive. Age, gender, HIV transmission route, number of ARV drugs, ARV drug class (PI/r, PI, or NNRTI) did not correlate in univariate analysis and were not included in the final model.

Conclusions: Higher CPE 2010 ranking effectively predicts CSF HIV suppression in patients with undetectable HIV-RNA in plasma. On the contrary, CPE predictive value does not seem the same in individuals with plasma active replicating virus. This could be explained by factors as HIV resistance, poor adherence or immune activation that may offset $\mathrm{CPE}$ in the context of virological failure. These results may have relevant implications for clinical strategy in order to characterize patients at higher risk of CSF/plasma discordant HIV-1 suppression.

\section{CO 29}

HIV ASSOCIATED NEUROCOGNITIVE DISORDERS: DIFFERENCES IN DIAGNOSTIC TOOLS AND MANAGEMENT STRATEGIES ACROSS ITALIAN HIV CLINICS

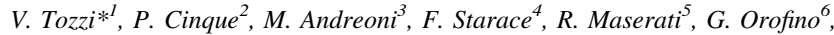
G. Orlando ${ }^{7}$, S. Carbonara ${ }^{8}$, A. Giacometti ${ }^{9}$, S. Bonora ${ }^{10}$, G. Parruti ${ }^{11}$, E. Quiros ${ }^{12}$, G. D'Ettorre ${ }^{13}$, G. Marchetti ${ }^{14}$, P. Lorenzini ${ }^{1}$, N.F. Di Campli ${ }^{15}$, A. Antinori ${ }^{1}$

${ }^{I}$ INMI L. Spallanzani IRCCS, Rome, Italy; ${ }^{2} H S R$, Istituto San Raffaele, Milan, Italy; ${ }^{3}$ Università Tor Vergata, Rome, Italy; ${ }^{4}$ Dipartimento Salute Mentale e Dipendenze Patologiche, AUSL, Modena, Italy; ${ }^{5}$ Policlinico San MatteoUniversità di Pavia, Pavia, Italy, ${ }^{6}$ Ospedale Amedeo di Savoia, Turin, Italy; ${ }^{7}$ Ospedale Luigi Sacco, Milan, Italy; ${ }^{8}$ Università di Bari, Bari, Italy;

${ }^{9}$ Università di Ancona, Ancona, Italy; ${ }^{10}$ Università di Torino, Turin, Italy;

${ }^{11}$ Ospedale di Pescara, Pescara, Italy; ${ }^{12}$ Università di Brescia, Brescia, Italy; ${ }^{13}$ Università La Sapienza, Rome, Italy; ${ }^{14}$ AO San Paolo-Università di Milano, Milan, Italy; ${ }^{15}$ ViiV Healthcare, Verona, Italy

Background: Although highly active antiretroviral therapy (HAART) has reduced the incidence of HIV-associated dementia (HAD), the overall prevalence of $\mathrm{HIV}$-associated neurocognitive disorders (HAND) has increased. Since treatment and prevention of HAND is becoming an increasing concern, management strategies for cognitive impairment in patients living with HIV are expected to play an important role in the near future. We performed a survey on management strategies in Italian HIV Clinics.

Methods: We performed a cross-sectional survey among Italian HIV Clinics using a standardized questionnaire. Investigated variables included: number of HIV-infected patients attending the Clinic, diagnostic tools for HAND (i.e. neuroimaging, virological and neuroimmunological equipments, neuropsychological testing, screening tools for cognitive impairment and psychiatric comorbidities), and management strategies.

Results: At the present writing, a total of 24 questionnaires from 24 HIV Clinics, were examined. Overall, the reported number of HIVinfected patient receiving HAART attending the HIV Clinics examined was 13.916 , corresponding to an estimated $23 \%$ of the Italian HIV-infected population currently treated. The availability of selected diagnostic tools for HAND in the 24 investigated HIV Clinics was as follows: brain MRI 23 HIV Clinics (96\%), functional MRI 14 (58\%), CSF HIV RNA plus genotypic resistance testing 23 (96\%), CSF PCR for opportunistic pathogens $24(100 \%)$, CSF markers of immunoactivation (i.e. MCP-1) $11(45 \%)$, full battery standardized neuropsychological testing 11 (45\%), International HIV Dementia Scale (HIDS) 8 (33\%), Mini Mental State Examination (MMSE) 11 (45\%).

Conclusions: The availability of virological and neuroimaging tools did not differ significantly among Italian HIV Clinics. By contrast, relevant differences existed in the accessibility to standardized neuropsychological evaluations and to other diagnostic tools for HAND including IHDS, and MMSE. In particular, the availability of standardized neuropsychological testing was largely underrepresented across the country. Since these diagnostic tools are currently recommended in National and International Guidelines for HAND diagnosis and treatment, efforts are needed to ensure their broader diffusion across the country. 
CO 30

THE KINETICS AND MAGNITUDE OF HIV-1 RNA RESPONSES TO ART IN THE CEREBROSPINAL FLUID AND PLASMA OF PATIENTS STARTING THERAPY

M. Viscione ${ }^{*}$, L. Sarmati ${ }^{1}$, A.R. Buonomini ${ }^{I}$, G. Maffongelli ${ }^{I}$, G. D'Ettorre ${ }^{2}$, G. Ceccarelli ${ }^{2}$, A. Bertoli ${ }^{1}$, V. Vullo ${ }^{2}$, C.F. Perno ${ }^{1}, M$. Andreoni $^{l}$

${ }^{I}$ Cattedra di Malattie Infettive, Tor Vergata, Rome, Italy; ${ }^{2}$ Cattedra di Malattie Infettive, La Sapienza, Rome, Italy

Background: Several studies have shown that antiretroviral therapies (ART) can suppress HIV-1 infection in the cerebrospinal fluid (CSF), although to varying degrees. However, some reports showed that, despite suppression of viral replication in plasma during ART, persistent viremia can be detected in the CSF. In order to compare the kinetics and magnitude of HIV-1 RNA responses to ART in the CSF and plasma we performed serial lumbar punctures (LPs) in 15 subjects with varying CD4 T lymphocyte depletion and ADC stages after the initiation of therapy.

Methods: LPs were performed before and after the initiation of ART (month 1, 3 and 6) in 15 naive HIV-1-infected subjects. HIV-RNA levels in plasma and CSF were detected at each time and residual viremia $(<1$ copy/ml $)$ was detected in samples with $<50$ copies $/ \mathrm{ml}$. The LP was not repeated in patients reaching undetectable level of HIV-RNA in CSF. Genotypic analysis of viral tropism was performed and the generated V3 sequences were then interpreted using geno2pheno website.

Results: At start of treatment mean CD4 cell count was 72 cells $/ \mu 1$ (range 9-220), plasma HIV-RNA was $5.63 \mathrm{log} \mathrm{cps} / \mathrm{ml}$ (range 4.19-6.42) and CSF HIV-RNA was $6.25 \mathrm{log} \mathrm{cps} / \mathrm{ml}$ (range 2.29-7.20). A X4 variant was detected in three patients in plasma and in one patient in CSF; one patient had a dual tropic virus either in plasma and in CSF. The mean central nervous system penetration-effectiveness (CPE) rank of ART was seven (range 6-10). A follow-up of at least 3 months was obtained in eight patients. Undetectable level of HIV-RNA was obtained in six patient in plasma (after a mean of 2.6 months) and in seven patients in CSF (after a mean of 2.1 months). In these patients the residual viremia was $16.8 \mathrm{cps} / \mathrm{ml}$ in plasma and $31.8 \mathrm{cps} / \mathrm{ml}$ in CSF. One patient had detectable HIV-RNA in plasma $(2.32 \mathrm{log} \mathrm{cps} / \mathrm{ml})$ and in CSF $(2.18 \log \mathrm{cps} / \mathrm{ml})$ after 6 months of therapy.

Conclusion: At the start of treatment the viral kinetics in the CSF and plasma are similar. Undetectable levels of HIV-RNA $(<40 \mathrm{cps} / \mathrm{ml})$ have been achieved before in CSF than in plasma. The presence of distinct HIV-1 populations and a higher residual viremia in the CSF than in plasma indicate that independent HIV-1 replication can occur in the central nervous system.

\section{CO 31}

APPLICATION OF GENOTYPIC TROPISM TESTING IN CLINICAL PRACTICE: VIROLOGICAL RESPONSE IN MULTI-EXPERIENCED HIV-POSITIVE PATIENTS TREATED WITH MARAVIROC-BASED THERAPY

S. Nozza* ${ }^{1}$, V. Svicher ${ }^{2}$, F. Canducci ${ }^{1}$, C. Alteri ${ }^{2}$, E. Boeri ${ }^{1}$, R. D'Arrigo ${ }^{2}$, A. Pignataro ${ }^{1}$, M. Clementi ${ }^{1}$, C.F. Perno ${ }^{2}$, A. Lazzarin ${ }^{1}$

${ }^{I}$ San Raffaele Scientific Institute, Milan, Italy; ${ }^{2}$ University of Roma Tor

Vergata, Department of Experimental Medicine, Rome, Italy

Background: Current guidelines recommend the use of coreceptor tropism assay whenever the use of CCR5 antagonist is considered. Trofile assay (Monogram Biosciences, CA, USA) has been used to screen patients in all CCR5 antagonists trials, but in clinical practice genotypic testing are being used. Most used algorithms to predict coreceptor usage by V3 sequencing are PSSM and geno2pheno (g2p) with False Positive Rate (FPR) of 5 and $10 \%$.

Methods: We retrospectively evaluated patients receiving Maraviroc for 48 weeks; coreceptor tropism was assessed by Trofile enhanced sensitivity version (ESTA). Gp120 V3 sequencing by g2p set at FPR of $10 \%$ were performed on stored plasma samples. Patients were evaluated at baseline and after 4, 12, 24, 36 and 48 weeks.
Virological, immunological and safety parameters were collected. Primary outcome of the study was virological response (percentage of patients with HIV-RNA $<50$ copies $/ \mathrm{ml}$ ) .

Results: Sixty-seven patients, 84\% males, age 46 (21-74), 16 (8-25) years of HIV-infection were screened in Maraviroc expanded access program. At baseline median CD4+ count was 194 cells/mmc (10-911), $11.8 \%$ (1.5-32.7), HIV-RNA $4.23 \log _{10} 4.23$ copies $/ \mathrm{ml}$ (1.88-5.96). ESTA indicated R5 in 40/67 (60\%) samples, non-R5 in 25/67 (37\%) and not determined in 2/67 (3\%). Geno2pheno indicated R5 in $72 \%$ and non-R5 in $28 \%$ of samples. High concordance $(84 \%)$ between $\mathrm{g} 2 \mathrm{p}$ and ESTA was reached in detection of R5-tropic viruses. Eleven samples were discordant: seven resulted non-R5 by ESTA and R5 by g2p; four R5 by ESTA and non-R5 by g2p. Virological responses were similar between the groups at different timepoints after the initiation of the therapy. Percentage of patients treated with maraviroc and with undetectable viral load is reported in the table. CD4 gain at week 48 was 222 cells/mmc (143-374) in ESTA group, 268 cells/mmc in g2p group (177-344).

\begin{tabular}{llllll}
\hline & $\begin{array}{l}\mathrm{W} 4 \\
(\%)\end{array}$ & $\begin{array}{l}\mathrm{W} 12 \\
(\%)\end{array}$ & $\begin{array}{l}\mathrm{W} 24 \\
(\%)\end{array}$ & $\begin{array}{l}\mathrm{W} 36 \\
(\%)\end{array}$ & $\begin{array}{l}\mathrm{W} 48 \\
(\%)\end{array}$ \\
\hline $\begin{array}{c}\text { ESTA R5 }^{\mathrm{a}} \\
(\mathrm{N}=40)\end{array}$ & 50 & 63 & 71 & 73 & 85 \\
$\mathrm{G}^{2} \mathrm{P} \mathrm{R} 5^{\mathrm{a}}(\mathrm{N}=36)$ & 57 & 73 & 80 & 71 & 84 \\
\hline
\end{tabular}

${ }^{a}$ Four patients treated with maraviroc retrospectively resulted nonR5 at g2p and they were not considered in the results for this group Conclusions: Virological responses were similar during 48 weeks of treatment with Maraviroc in multi-treatment experienced population. These data confirm the utility of V3 genotyping assay to select patients for CCR5 antagonists treatment. Prospective and comparative clinical study between ESTA and g2p should be performed.

\section{CO 33}

RILPIVIRINE IS COMING: HOW WILL IT IMPACT ON HIV-1INFECTED ITALIAN POPULATION?

R. Rossotti ${ }^{1}$, L. Fonte ${ }^{2}$, G. Meini ${ }^{3}$, F. Maggiolo ${ }^{4}$, S. Di Giambenedetto ${ }^{5}$, M. Pecorari ${ }^{6}$, P. Corsi ${ }^{7}$, L. Monno ${ }^{8}$, A. Giacometti ${ }^{9}$, D. Francisci $^{\text {IO }}$, M. Setti ${ }^{11}$, R. Maserati ${ }^{2}$, P. Bonfanti ${ }^{l}$, A.Orani ${ }^{l}$, M. Zazzi ${ }^{3}$

${ }^{I}$ Department of Infectious Diseases, AO "A. Manzoni", Lecco, Italy;

${ }^{2}$ Direzione Medica di Presidio, AO "A. Manzoni", Lecco, Italy; ${ }^{3}$ Section of Microbiology, Department of Molecular Biology, University of Siena, Siena, Italy; ${ }^{4}$ Division of Infectious Diseases, Ospedali Riuniti, Bergamo, Italy; ${ }^{5}$ Clinic of Infectious Diseases, Catholic University of the Sacred Heart, Rome, Italy; ${ }^{6}$ Laboratory of Microbiology and Virology, Modena University Hospital, Modena, Italy; ${ }^{7}$ Unit of Infectious Diseases, Careggi University Hospital, Florence, Italy; ${ }^{8}$ Clinical Infectious Diseases, Bari University Hospital, Bari, Italy; ${ }^{9}$ Institute of Clinical Infectious Diseases, Ancona University Hospital, Ancona, Italy; ${ }^{10}$ Division of Infectious Diseases, Department of Experimental Medicine and Biochemical Sciences, University of Perugia, Perugia, Italy;

${ }^{11}$ Division of Clinical Immunology, Department of Internal Medicine, University of Genoa Medical School, Genoa, Italy; ${ }^{12}$ HIV/AIDS Outpatient Clinic Infectious Diseases Department, Foundation "IRCCS Policlinico San Matteo Hospital", Pavia, Italy

Introduction: Rilpivirine (TMC278, RPV) is a novel NNRTI under evaluation in treatment-naïve subjects in two trials started in 2008. Given its different mutational pattern, clinicians are waiting for RPV even for experienced subjects with poor therapeutic options. There are few evidences about RPV mutational profile in vivo: E138K is the only resistance-associated mutation (RAM) that affects the susceptibility to RPV. Data in vitro describe other RAMs (L100I, K101E, V106I, Y181C/I, M230I) that seem to act as primary mutations. Aim of our study: Compare the prevalence of these RAMs in Italian naïve and experienced population; assess if a previous exposure to NNRTIs could represent a risk to develop RPV-associated RAMs. 
Methods: The retrospective analysis is performed using the ARCA database; it contains data on genotypic resistance and therapy of HIVinfected subjects from Infectious Diseases Units throughout Italy. The database was queried about the resistance pattern in naïve subjects from 2008 to June 30, 2010; prevalence of primary RPV-associated and firstgeneration NNRTI-associated (K103N, Y181C, G190A/S/E) RAMs was evaluated. Experienced population was analyzed in the whole database. Comparisons were performed with Chi-square test. Logistic regression analysis was performed to assess if a previous exposure to another NNRTI represents a risk factor to develop RPV-associated RAMs. Data from subjects treated for 4 weeks were included.

Results: The ARCA database query selected 21,677 tests: 9,066 from naïve (3,428 from 2008) and 11,187 from experienced subjects; 1,424 have no data about therapy and were excluded. Among naïves, prevalence of RPV-associated RAMs is $8.4 \%$, lower than $14.1 \%$ for the other NNRTIs ( $\mathrm{p}<0.001$ ); similar results for E138K and K103N (0.6 vs. 8.0\%, $\mathrm{p}<0.001$; Fig. 1). Prevalence of these RAMs among experienced subjects is shown in Fig. 2. EFV has an increased risk to develop E138K $(\mathrm{p}=0.044)$. Results for ETV and DLV are shown even if the statistic power is not adequate given their weak presence in the database (Table 1).

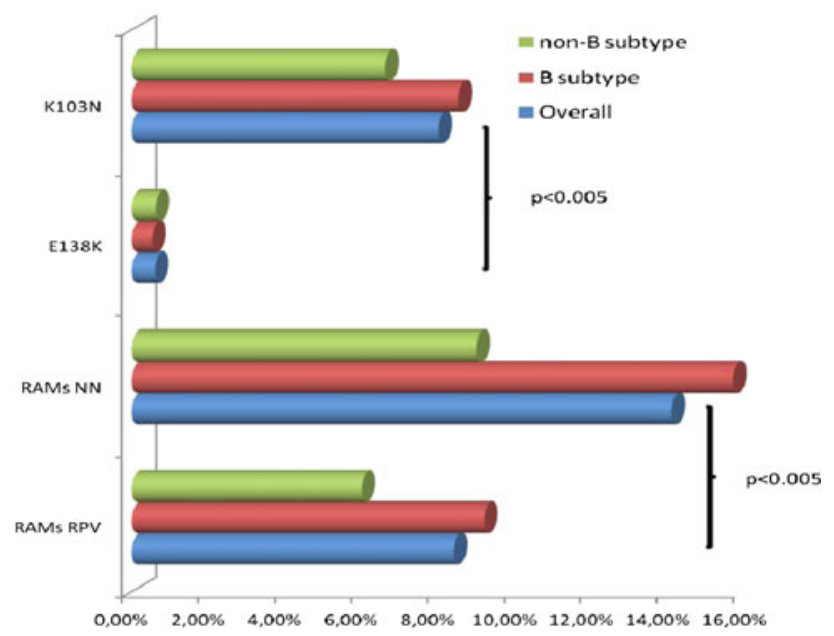

Fig. 1 Comparison between the prevalence of first generation NNRTI-associated (K103N, Y181C and G190A/S/E) and RPVassociated (L100I, K101E, V106I, E138K, Y181C/I, M230I) mutations in the Italian HAART-naïve population; comparison between the most important EFV-and RPV-associated mutations (K103N vs. E138K). Data are shown for the overall Italian naïve population and split between B and non-B subtypes (Chi-square test)

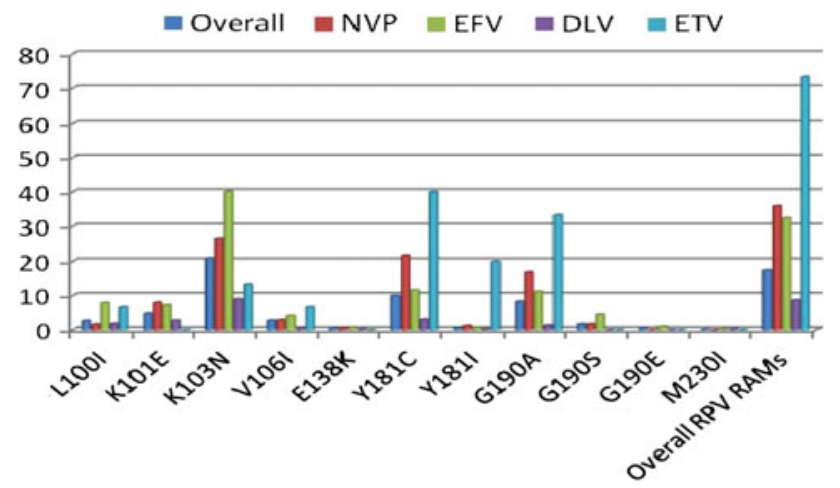

Fig. 2 Prevalence of NNRTI-associated (either first and secondgeneration) RAMs in NNRTI-experienced patients (\%)
Discussion: Italian naïve and experienced populations are more frequently susceptible to RPV than to EFV. EFV is a risk factor to develop E138K, while NVP has no effect. A sequencing strategy seems safer when the patient is pre-exposed to NVP than to EFV (except for Y181C/I). A clinical validation of the meaning of these RAMs is needed to understand the real effect of these mutations.

Table 1 Risk of developing a primary RPV-associated RAM for after a previous exposure to another NNRTI for at least 4 weeks (logistic regression analysis)

\begin{tabular}{|c|c|c|c|}
\hline & Odds ratio & $95 \% \mathrm{Cl}$ & $\mathrm{p}$ \\
\hline \multicolumn{4}{|l|}{$\mathrm{K} 138 \mathrm{E}$} \\
\hline NVP & 0.864 & $0.455-1.640$ & $>0.05$ \\
\hline EFV & 2.569 & $1.025-6.444$ & 0.044 \\
\hline DLV & ns & & \\
\hline ETV & ns & & \\
\hline \multicolumn{4}{|l|}{ L100I } \\
\hline NVP & 0.392 & $0.274-0.559$ & $<\mathbf{0 . 0 0 1}$ \\
\hline EFV & 13.243 & 9.635-18.201 & $<\mathbf{0 . 0 0 1}$ \\
\hline DLV & 0.406 & $0.056-2.954$ & $>0.05$ \\
\hline ETV & 1.601 & $0.207-12.361$ & $>0.05$ \\
\hline \multicolumn{4}{|l|}{ K101E } \\
\hline NVP & 1.767 & $1.422-2.196$ & $<\mathbf{0 . 0 0 1}$ \\
\hline EFV & 0.850 & $0.695-1.041$ & $>0.05$ \\
\hline DLV & ns & & \\
\hline ETV & ns & & \\
\hline \multicolumn{4}{|l|}{ V106I } \\
\hline NVP & 1.040 & $0.814-1.329$ & $>0.05$ \\
\hline EFV & 1.645 & $1.303-2.075$ & $<\mathbf{0 . 0 0 1}$ \\
\hline DLV & ns & & \\
\hline ETV & 2.598 & $0.337-20.043$ & $>0.05$ \\
\hline \multicolumn{4}{|c|}{ Y181C } \\
\hline NVP & 5.943 & $4.901-7.207$ & $<\mathbf{0 . 0 0 1}$ \\
\hline EFV & 0.349 & $0.301-0.405$ & $<\mathbf{0 . 0 0 1}$ \\
\hline DLV & 0.197 & $0.048-0.814$ & 0.025 \\
\hline ETV & 6.806 & $2.283-20.288$ & 0.001 \\
\hline \multicolumn{4}{|l|}{ Y181I } \\
\hline NVP & 3.432 & $1.726-6.825$ & $<\mathbf{0 . 0 0 1}$ \\
\hline $\mathrm{EFV}$ & 0.423 & $0.242-0.739$ & 0.003 \\
\hline DLV & ns & & \\
\hline ETV & 33.513 & $8.511-131.957$ & $<\mathbf{0 . 0 0 1}$ \\
\hline \multicolumn{4}{|l|}{ M230I } \\
\hline NVP & 0.889 & $0.230-3.442$ & $>0.05$ \\
\hline EFV & 1.447 & $0.408-5.131$ & $>0.05$ \\
\hline DLV & 21.700 & $2.695-174.700$ & 0.004 \\
\hline ETV & ns & & \\
\hline
\end{tabular}




\section{CO 34}

DUAL/MIXED (BUT NOT X4) TROPIC-HIV-1 ISOLATES CAN REPLICATE IN HUMAN PRIMARY MACROPHAGES AND ARE INHIBITED BY CCR5-INHIBITORS

M. Surdo ${ }^{2}$, E. Balestra ${ }^{2}$, P. Saccomandi ${ }^{2}$, F. Di Santo ${ }^{2}$, V. Svicher ${ }^{2}$, C. Alteri ${ }^{2}$, V. Cento ${ }^{2}$, M. Pollicita ${ }^{2}$, F. Scopelliti ${ }^{2}$, M. Andreoni ${ }^{2}$, S. Aquaro ${ }^{1}$,

F. Ceccherini-Silberstein ${ }^{2}$, C.F. Perno ${ }^{2}$

${ }^{1}$ Dipartimento di Farmaco-Biologia, Rende, CS, Italy; ${ }^{2}$ Dipartimento di Medicina Sperimentale e Scienze Biochimiche Università di Roma Tor

Vergata, Rome, Italy

Background: Human monocyte-derived macrophages (MDM) are widely involved in HIV-pathogenesis and clinics. Despite the importance of the inhibition of the entry and replication of the virus into these reservoirs, in vitro studies with clinical isolates from patients are so far limited. The aim of the study was to analyze the replication capacity and susceptibility to maraviroc (MVC, a CCR5inhibitor) of HIV-1 clinical isolates with different tropism in human primary MDM.

Methods: 23 HIV-1 isolates were genotypically and phenotypically characterized. The entire V3 sequence was successfully obtained from all isolates, and genotypic tropism prediction was obtained using PSSM (set at -5.96 cut-off) and Geno2Pheno (G2P; set at FPR $=10 \%$ ) algorithms. Phenotypic tropism was evaluated by multiple cycles assay on U87MG-CD4+-CCR5+-/CXCR4+-expressing cells. The replication capacity and susceptibility to MVC at different concentrations $(200,20$ and $2 \mathrm{nM})$ were investigated in human primary MDM. AMD3100 was used as X4-tropic strain inhibitor.

Results: Phenotypic-assay reported pure R5-tropic viruses in 6 $(26.1 \%)$ isolates, dual/mixed (D/M)-tropic viruses in $15(65.2 \%)$, and pure X4-tropic viruses in only $2(8.7 \%)$. Among $15 \mathrm{D} / \mathrm{M}$-tropic isolates, 8 showed a remarkably higher replication-efficacy in CCR5expressing cells (R5+/X4), 4 in CXCR4-expressing cells (R5/X4+), while 3 showed similar replication in both cell types (R5/X4). Genotypic assay was $91.4 \%$ concordant with the phenotypic test. All isolates with FPR $>20 \%$ were phenotypically defined as R5 - or R5+l $\mathrm{X} 4$-tropic viruses (12/12). They all replicated with higher efficiency in MDM, compared with isolates with FPR $<20 \%$ (median 9,953 pg/ $\mathrm{ml}$; IQR 14,123-3,947 vs. $304 \mathrm{pg} / \mathrm{ml}$; IQR 1,766-<125, respectively, $\mathrm{p}=0.0004)$. Of interest, only $45.5 \%(5 / 11)$ of strains with FPR $<20 \%$ replicated in MDM, while all 23 isolates efficiently replicated in lymphocytes cultures. MVC was highly effective against all tested isolates with FPR $>20 \% \quad(n=6$, including $1 \mathrm{R} 5+/ \mathrm{X} 4)$, showing a median [IQR] inhibition of the viral replication of $99.5 \%$ [99.9-98.9] at $200 \mathrm{nM}, 98 \%$ [99.7-92.4] at $20 \mathrm{nM}$, and $56.8 \%$ [76.4-44.6] at $2 \mathrm{nM}$. Of interest, also $1 \mathrm{R} 5 / \mathrm{X} 4$ and $1 \mathrm{R} 5 / \mathrm{X} 4+$ tested, both with FPR $<5 \%$, that well replicated were inhibited by MVC (median [IQR] inhibition of $94.6 \%$ [97.3-91.8] at $200 \mathrm{nM}, 90.1 \%$ [95.1-85.2] at $20 \mathrm{nM}, 70.8 \%$ [79.6-62.0] at $2 \mathrm{nM}$ ). By contrast, and as expected, R5- tropic viruses were not inhibited by AMD3100 at concentration of $1 \mu \mathrm{g} / \mathrm{ml}\left(100-1,000\right.$-fold $\left.>E_{50}\right)$; the same high concentration inhibited replication in MDM of D/M-tropic viruses only at a median of $66.6 \%$ (IQR 73.0-33.3), far lower than the activity of MVC $200 \mathrm{nM}$ (100-fold $\left.>\mathrm{EC}_{50}\right)$ observed on the same D/M-viruses.

Conclusions: D/M-tropic HIV-1 isolates better replicate in MDM if characterized by FPR values $>20 \%$, while all X4-tropic isolates tested didn't replicate at all. Both R5- and D/M-viruses were efficiently inhibited by MVC in MDM (but not by AMD3100). Thus, the presence of an even minor R5 tropism remains mandatory for replication in MDM, yet CCR5 inhibitors are highly effective against all viruses with these dual characteristics. Overall data suggest that CCR5antagonists may stand as a therapeutic option also against D/M-tropic viruses.
CO 35

PREVALENCE OF ETRAVIRINE (ETR)-RESISTANCE ASSOCIATED MUTATIONS AT NNRTI FAILURE AND PREDICTORS OF RESISTANCE TO ETR IN A LARGE ITALIAN RESISTANCE DATABASE (ARCA)

S. Rusconi ${ }^{\text {I }}$, F. Adorni ${ }^{2}$, B. Bruzzone ${ }^{3}$, A. Di Biagio ${ }^{3}$, G. Meini ${ }^{4}$, A. $^{2}$

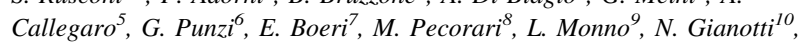
L. Butini ${ }^{11}$, L. Galli ${ }^{12}$, E. Polilli ${ }^{13}$, M. Galli ${ }^{1}$ on behalf of the ARCA

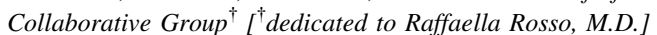

${ }^{I}$ Sezione di Malattie Infettive e Immunopatologia, Dipartimento di Scienze Cliniche “Luigi Sacco", Universita' degli Studi di Milano, Milan, Italy; ${ }^{2}$ ITBCNR, Segrate, Milan, Italy; 'Malattie Infettive \& Microbiologia, Universita' degli Studi di Genova, Genoa, Italy; ${ }^{4}$ Istituto di Microbiologia \& Virologia, Universita' degli Studi di Siena, Siena; ${ }^{5}$ Microbiologia \& Virologia, Ospedali Riuniti di Bergamo, Bergamo, Italy; ${ }^{6}$ Istituto di Virologia, Università degli Studi di Bari, Bari, Italy; ${ }^{7}$ Virologia, Istituto Scientifico San Raffaele, Milan, Italy; ${ }^{8}$ Virologia, Policlinico Universitario, Modena, Italy; ${ }^{9}$ Clinica Malattie Infettive, Università degli Studi di Bari, Bari, Italy; ${ }^{10}$ Clinica Malattie Infettive, Istituto Scientifico San Raffaele, Milan, Italy; ${ }^{11}$ Immunologia Clinica e Tipizzazione Tessutale, Università Politecnica delle Marche, Torrette di Ancona, Ancona, Italy; ${ }^{12}$ Malattie Infettive, Ospedale Pediatrico Meyer, Florence, Italy; ${ }^{13}$ Virologia, Ospedale Santo Spirito, Pescara, Italy; [dedicated to Raffaella Rosso, M.D.]

Background: Etravirine (TMC125, ETR) is the newest nonnucleoside reverse transcriptase inhibitor (NNRTI) designed to be active against both wild-type and NNRTI-resistant HIV. Three algorithms have been developed to interpret ETR resistance: Monogram (MGR), Tibotec (TBT), and enhanced MGR (ENH). We investigated the prevalence of drug resistance mutations associated to NNRTI-based regimens failure and the predictors of resistance to ETR among subjects included in a large Italian resistance database.

Material and methods: From the Italian database ARCA ( http://www.hivarca.net), we selected 5,547 sequences from 3,047 subjects up to 29 December 2010. Among these individuals, $39 \mathrm{had}<18$ (P) years and 2,815 were $\geq 18$ (A) years-old. These subjects failed their current NNRTI treatment, were three-class experienced and had been exposed to NNRTI $\geq 3$ months. Complete treatment history, HIV-1 RNA $>1,000 \mathrm{cp} / \mathrm{mL}$ at failure, CD4 counts within 30 days before the genotypic resistance test were available. Binomial logistic regression analysis was carried out and odds ratio (AOR or OR [CI 95\%]) were expressed. All data were adjusted for CD4 counts and HIV-1 RNA levels.

Results: 1,827 A subjects (64.9\%) and 32 P subjects (82.1\%) harboured virus with at least one ETR mutation included in at least one score. Among the A subjects with at least one ETR mutation, mutations more frequently detected were Y181C (18.5\%), G190A (15.1\%), and V179I (11.2\%). Among P subjects, V179I, Y181C, and G190A were present in $30.8,28.2$ and $23.1 \%$, respectively. Univariate analysis revealed an increased risk in the pediatric population (vs. adult population) for exceeding cut-off values of ETR resistance with all three algorithms: MGR $>3$ OR $2.10(1.11-3.94) \mathrm{p}=.022$, TBT $>2$ OR $2.56(1.36-4.82)$ $\mathrm{p}=.004$, and $\mathrm{ENH} \geq 4$ OR $2.44(1.28-4.64) \mathrm{p}=.007$. Multivariate analysis revealed an increased risk of developing TBT $>2$ for NNRTI exposure, ENH $\geq 4$ for NNRTI and EFV exposure in P subjects; NVP exposure and higher $\left(\geq 3.5 \log _{10}\right)$ HIV-RNA values for all three algorithms in A subjects, whereas CD4 $\geq 200 / \mu \mathrm{L}$ appeared to be protective. Conclusions: The DUET studies showed that $\geq 3$ ETR-associated mutations were required to impair the efficacy of the drug and Y181C/V, V179F and G190S had the most pronounced effect on response. The prevalence of $\mathrm{Y} 181 \mathrm{C}$ mutation was higher in $\mathrm{P}$ versus A subjects (28.2 vs. $18.5 \%$ ) together with G190A and V179I. The risk to be ETR resistant, according to all algorithms, was more than double for $\mathrm{P}$ versus A subjects, probably due to a more extensive use of NNRTI and an incomplete virological control. Determinants of genotypic resistance to ETR were higher HIV-1 RNA values and 
greater NVP exposure in A subjects; a detrimental effect of NNRTI and EFV exposure was shown in P subjects. Higher levels of immune competence were protective for future development of ETR genotypic resistance.

\section{CO 36}

INTRACELLULAR ACCUMULATION OF RITONAVIR ASSOCIATED WITH DIFFERENT BOOSTED PIS

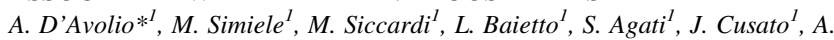

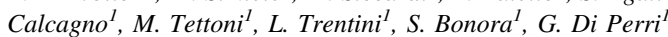

${ }^{1}$ Università degli Studi di Torino, Torin, Italy

Background: Ritonavir (RTV), although widely used at low doses as a boosting agent of the other protease inhibitors (PIs), is known to be associated with metabolic complications and gastrointestinal disturbances. However, rate of accumulation of RTV within the cells is still debated due to scarce data and methodological limitations. Therefore, our aim was to evaluate intracellular RTV penetration when used with different boosted PIs in the clinical setting.

Materials and methods: Patients administered with standard dose of boosted Atazanavir (ATV)/RTV (300/100 qd), Darunavir (DRV)/ RTV (600/100 bid), Lopinavir (LPV)/RTV (400/100 bid) and Tipranavir (TPV)/RTV 500/200 bid) were considered. Main inclusion criteria were no concomitant interacting drugs, no hepatic or renal function impairment, and self-reported adherence $>95 \%$. Blood sampling at the end of dosing interval (Ctrough) was performed after written informed consent given. PBMCs-associated and plasma RTV and PIs concentrations were measured by a validated HPLC-MS method and a validated HPLC-PDA, respectively. Cell count and mean cell volume were performed by a Coulter Counter instrument and data used for calculate total PBMC volume. Median value of individual measurements was considered. Statistical analysis was performed by Mann-Whitney and Spearman Rank. Values were expressed as $\mathrm{ng} / \mathrm{ml}$.

Results: 61 patients were enrolled, whose 11 were administered with TPV/RTV, 16 with DRV/RTV, 24 with ATV/RTV, 10 with LPV/ RTV. Median $( \pm$ SD) RTV intracellular concentrations were 1,137 $( \pm 745) \mathrm{ng} / \mathrm{ml}$ for TPV recipients, $2,245( \pm 1,521) \mathrm{ng} / \mathrm{ml}$ for DRV, $668( \pm 687) \mathrm{ng} / \mathrm{ml}$ for ATV, and 1,992 $( \pm 619) \mathrm{ng} / \mathrm{ml}$ for LPV. Differences of median RTV intracellular concentrations were significant between ATV and DRV ( $p<0.001)$, ATV and LPV $(\mathrm{p}<0.001)$, TPV and DRV $(p=0.003)$ and TPV and LPV $(p=0.017)$. Median RTV intracellular/plasma concentrations ratios were 9.16 for ATV recipients, 7.32 for DRV, 5.03 for TPV and 6.83 for LPV. Median PI intracellular/plasma concentrations ratio were 2.44 for ATV, 0.07 for DRV, 0.14 for TPV and 0.40 for LPV.

Conclusions: Our study showed a general rate of RTV intracellular accumulation higher as previously reported, probably due to the more accurate calculation of intracellular concentrations (use of mean individual PBMCs volume). This rate showed to vary according to concomitant boosted PI, suggesting a role of the latter in affecting RTV penetration. ATV recipients showed lowest RTV concentration, as expected from $100 \mathrm{mg}$ qd dosing, although rate of intracellular accumulation was the highest (9-fold higher than in plasma). Among RTV bid administered patients, TPV recipients, although dosed with $200 \mathrm{mg}$ bid, showed the lowest RTV concentrations. Further clinical study are warranted in order to elucidate inter-individual differences and clinical implications of RTV intracellular penetration.

\section{CO 37}

FINAL RESULTS OF AN OUTREACH PROGRAM OF HIV RAPID TESTING AMONG MARGINALIZED PEOPLE LIVING IN ROME, ITALY

P. Scognamiglio $*^{\prime}$, G. Chiaradia ${ }^{I}$, M.R. Sciarrone $e^{I}$, M.R. Capobianchi ${ }^{l}$, E.

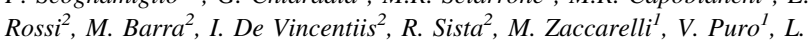
Ceccarini $^{2}$, G. Ippolito ${ }^{1}$, E. Girardi ${ }^{l}$

${ }^{1}$ Istituto Nazionale Malattie Infettive "L.Spallanzani" IRCCS, Rome, Italy;

${ }^{2}$ Fondazione "Villa Maraini" Onlus-Roma, Rome, Italy
Background: HIV rapid testing technology represents an opportunity to facilitate early HIV diagnosis in population at high risk for HIV infection with poor access to care. Aim of this project was to assess the feasibility and effectiveness of an outreach HIV rapid testing program provided to marginalized people living in Rome

Methods: The study was conducted within a street-based HIV risk reduction program run by a NGO among marginalized people (drug users, sex workers, homeless, immigrants) living in Rome. Between February and August 2010, each individual attending to the mobile unit was offered an anonymous HIV rapid test (VIKIA HIV ${ }^{\circledR} 1 / 2$ ), providing a finger-prick blood sample and filling out a questionnaire while waiting. People already HIV diagnosed or aged $<18$ years were excluded. Individuals with a reactive result were referred to a specialized outpatient unit for confirmatory testing and medical care.

Results: We approached 1,058 individuals: 30 were excluded (25 HIV+); 1,028 were offered a rapid HIV testing and 323 refused. The most common reason for refusal was a recent test $(56 \%)$; only eight individuals declared they did not trust rapid test. We tested 705 people with a median age of 33 years, $68.4 \%$ males, $40 \%$ injecting drug users (IDUs), 24\% percent non-injecting drug users (nIDUs) and $23 \%$ were migrants not DU. $43 \%$ were never HIV tested before. Eight individuals (one point one percent of tested people), all DUs, had a preliminary positive result: 7 males (5 IDU; 2 NIDU) and 1 female (IDU); 3 had never been tested before. Only one male IDU attended our outpatient unit and was confirmed as HIV infected. Stratifying the results by target population (DU; migrants no DU; Italians no DU) the lowest testing and acceptance rate were found among DUs, significantly lower than those found among migrants but all eight HIV positive individuals were DUs. Moreover $28 \%$ of DUs, $68 \%$ of migrants and $74 \%$ of non drug users Italians had never been tested for HIV before.

Conclusions: Our study showed an high acceptance rate of HIV rapid test. In $43 \%$ of cases this opportunity represented the first approach to HIV testing but the high proportion of failure to return for confirmatory test underlies the difficulty to link to care intravenous drug users.

\section{CO 38}

INCREASING TREND IN HIV-1 INCIDENCE AMONG MEN WHO HAVE SEX WITH MEN IN ROME, ITALY

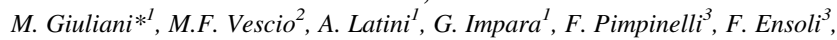
G. Palamara ${ }^{1}$, G. Rezza ${ }^{2}$, A. Di Carlo ${ }^{l}$

${ }^{I}$ UOC Dermatologia Infettiva, Istituto Dermatologico S.Gallicano (IRCCS),

Rome, Italy; ${ }^{2}$ Dipartimento Malattie Infettive, Parassitarie e Immunomediate, Istituto Superiore di Sanità, Rome, Italy; ${ }^{3}$ Laboratorio di Patologia Clinica e Microbiologia. Istituto Dermatologico S. Gallicano (IRCCS), Rome, Italy

Introduction: Since the early 2000 s, data from national surveillance systems and observational studies seem to suggest that, in many European countries, HIV-1 infection continues to affect disproportionately men who have sex with men (MSM).

Aims: To assess HIV-1 incidence trend among a large cohort of MSM attending a structured sexually transmitted infections (STI) screening program (COROH Project) in Rome, Italy.

Methods: All MSM tested for HIV-1 antibodies more than once after 1984 were included in the study. Incidence rates were calculated using the person year approach. Time at risk was defined as the difference between the date of first blood sample and the date of last blood sample. Because we do not know exactly when seroconversion occurred, we assume that seroconverted individuals were at risk for half the interval between the last negative and the first positive examination. 
Result: During the study period, 1,869 MSM were tested more than once and were included in the cohort study. The median number of HIV tests received by MSM was 4.0 (range 2-29) and the median follow-up time was 2.76 years. During the study period, 347 HIV incident infections were observed for an overall IR of $4.0(95 \% \mathrm{CI}$ $3.6 ; 4.5)$ per $100 \mathrm{p} / \mathrm{y}$. No differences in age were found between seroconverters and non-seroconverters individuals $(35.2[ \pm 9.4]$ years vs. $35.4[ \pm 11.0])$. After the 1992 , the incidence rate stably decreases from $11.3(95 \%$ CI $8.0 ; 16.1)$ per $100 \mathrm{p} / \mathrm{y}$ in 1992 to $1.6(95 \% \mathrm{CI}$ $0.6 ; 3.8)$ per $100 \mathrm{p} / \mathrm{y}$ in 2001. From 2002 the trend showed a progressive increase up to 2009, when incidence peaked at 25.3 (95\% CI $16.0 ; 40.2)$ per $100 \mathrm{p} / \mathrm{y}$.

Conclusion: Also confirming our previous observations in this at-risk population, the HIV-1 incidence among MSM resident in Rome, Italy, tended to increase after 2001 and up to 2009. The trend seem consistent to the reemergence of other STI in the same group. Nevertheless, further analysis of the $\mathrm{COROH}$ Project dataset are in progress to better define the spectrum of risk factors associated with this increase.

\section{CO 39}

CHANGES IN SURVIVAL OF HIV-SEROCONVERTED PATIENTS ENROLLED IN THE IHS STUDY FROM 1985 TO 2007

M. Dorrucci ${ }^{*}{ }^{l}$, M. Giuliani ${ }^{I}$, G. Rezza $a^{I}$ and the Italian HIV-Seroconversion Study Group ${ }^{2}$

${ }^{I}$ Dipartimento di Malttie Infettive, Parassitarie e Immunomediate (MIPI), Istituto Superiore di Sanità, Rome, Italy; ${ }^{2}$ Italian HIV-Seroconversion Study Group, Italy

Introduction: After the introduction of combination antiretroviral therapy (cART) for HIV-1 infection, longitudinal cohort study represents a gold standard approach to better define the course of disease also in treated populations.

Objectives: To assess changes over time in survival following HIVseroconversion (SC) in the era of cART, providing updated survival estimates.

Methods: Using data from patients with estimated dates of HIV-SC enrolled in Italian HIV-seroconversion study (IHSS) from 1985 to 2007, we studied time from SC to death from any cause, applying Cox models adjusted for prognostic variables. Kaplan-Meier method was used to estimate the expected survival in different calendar periods considering staggered entries.

Results: During the study period, 2,278 patients were considered with 29,824 p-y of follow-up. A total of 644 (28\%) deaths were observed. The hazard ratio (HR) of death, compared with the period 1985-1995, decreased over time to 0.51 (95\% CI 0.41-0.63), $0.18(0.14-0.25)$ and $0.12(0.09-0.18)$ in 1996-1999, 2000-2003 and 2004-2007, respectively. These findings were similar when applying multiple Cox models considering age at SC, gender and HIV-transmission group. In 2000-2007, the proportion of individuals expected to survive 5, 10 and 15 years following SC was 97,92 and $87 \%$, respectively.

Conclusions: Since 1985, survival after HIV-SC has continued to improve over time in the Italian cohort of HIV-seroconverters. From 2000 , such high survival expectations in this population, underlie the positive role of early diagnosis in the clinical management of HIVinfected individuals.

${ }^{2}$ Italian HIV-Seroconvertion Study Group includes: Umberto Tirelli, Aviano, Italy; Gioacchino Angarano, Bari, Italy; Vincenzo Colangeli, Bologna, Italy; Raffaele Pristerà, Bolzano, Italy; Giampiero Carosi, Brescia, Italy; Adriano Lazzarin, Milan, Italy; Massimo Galli, Milan, Italy; Francesco Alberici, Piacenza, Italy; Maria Alessandra Ursitti, Reggio Emilia, Italy; Mauro Zaccarelli, Rome, Italy; Roberto Cauda, Rome, Italy; Massimo Giuliani, Guido Palamara, Rome, Italy; Caterina Fimiani, Rome, Italy; Giovanni Di Perri, Turin, Italy
CO 40

HIV SURVEILLANCE SYSTEM AND LATE PRESENTERS IN

EMILIA-ROMAGNA, 2006-2009

E. Massimiliani $*^{I}$, A. Mattivi ${ }^{I}$, L. Droghini ${ }^{I}$, A.C. Finarelli ${ }^{l}$ and the SorvHIVRER workgroup ${ }^{2}$

${ }^{I}$ Servizio Sanità Pubblica, Assessorato Politiche per la Salute, Regione EmiliaRomagna, Bologna, Italy; ${ }^{2}$ Clinica Malattie Infettive di Bologna, Cesena,

Ferrara, Forli, Imola, Modena, Parma, Piacenza, Reggio Emilia, Ravenna, Rimini, Emilia-Romagna

Background: Following the introduction of the National HIV Surveillance System in 2008, Emilia-Romagna Region implemented its own system (SorvHIV-RER), with the collaboration of the Infectious Diseases Units working on the regional area. Collected data allowed to examine characteristics of people with a new HIV diagnosis and to analyse risk factors associated with patients presenting at later stages of HIV disease, according to definition of Late Presenters (already AIDS presentation and/or low CD4+ cells count at diagnosis).

Methods: Each new HIV diagnosed case, with at least one CD4+ lymphocyte count and a recorded stage of HIV disease, identified among residents in Emilia-Romagna from 2006 and 2009 was included in the observation. Descriptive statistics were used to describe cases characteristics, time trends and spatial distributions. A multivariate logistic regression analysis was also performed to examine factors associated with being Late Presenter patient. Definition used were: Late Presenter (LP), identifying new HIV diagnosis with a CD4 count below 350 cells/ $\mu \mathrm{L}$ or presenting with an AIDSdefining event (ADE), regardless of the CD4 cell count and Advanced HIV Disease (AHD) if they had a CD4 count below 200 cells/ $\mu \mathrm{L}$ or an ADE, regardless of the CD4 cell count.

Results: 1,552 new HIV diagnoses were reported in SorvHIV-RER system during the study period, determining an incidence rate of 9.1 cases per 100,000 inhabitants. Men represented $72.0 \%$ of diagnosed cases, the median age was 39 years and the proportion of persons born outside Italy was $29.0 \%$. Sexual transmission occurred in $83.8 \%$ of total cases. Out of these, $55.5 \%$ was related to heterosexual contacts (HC) and $28.3 \%$ to men who have sex with men (MSM). Injecting drug users (IDU) represented $5.5 \%$ of all HIV persons. Among all new HIV diagnoses $48.1 \%$ were LP and $32.7 \%$ were in a condition of AHD. Older age, foreign born and heterosexual transmission represented significant determinants for being classified as a Late Presenter (age per a 5 years increase: OR 1.34, [1.26-1.41] 95\% CI; Foreign: OR 2.37, [1.81-3.10] 95\% CI; HC: OR 1.58, [1.11-2.24] $95 \% \mathrm{CI}$ ) or to have an AHD (age per a 5 years increase: OR 1.27, [1.21-1.34] 95\% CI; Foreign: OR 2.03, [1.54-2.67] 95\% CI; HC: OR 1.60, [1.09-2.34] 95\% CI).

Discussion: In Emilia-Romagna about 50\% of people with a new HIV diagnosis during the observed years were Late Presenters and needed to undergo antiretroviral therapy (ART); one-third were at an advanced stage of immune deficiency, requiring higher costs in care. In most cases, benefits of ART were limited in immune-restoration and presented a higher mortality during the advanced stage of disease. Since transmission through sexual contact accounted for most HIV new diagnoses, efforts are needed to promote safe behaviours and to encourage HIV testing, in order to reduce the HIV incidence and to early detect new HIV cases.

\section{CO 41}

HEALTH-CARE DELIVERY CHARACTERISTICS OF CLINICAL CENTERS ARE CORRELATED WITH SURVIVAL OF ITALIAN HIV PATIENTS

R. Murri ${ }^{1}$, A. Ammassari ${ }^{2}$, M.P. Trotta ${ }^{2}$, A. Antinori ${ }^{2}$, P. Lorenzini ${ }^{2}$,

A. D'Arminio Monforte ${ }^{3}$

${ }^{1}$ Università Cattolica S Cuore, Rome, Italy; ${ }^{2}$ INMI Spallanzani, Rome, Italy;

${ }^{3}$ Azienda Ospedaliera-Polo Universitario San Paolo, Milan, Italy 
Background: Delivery of health care strongly varies throughout clinical centers. Previous published studies have demonstrated that some health care delivery characteristics, such as increased physician experience as well as higher pharmacy refill rates, are correlated with improved survival.

Objective and methods: Aim of the study was to evaluate the risk of death or AIDS-defining event in a cohort of HIV-infected patient population according to different health-care delivery characteristics. For this purpose, a cross-sectional survey was carried out in the 39 clinical centers participating in the Italian Cohort Naïve Antiretrovirals (ICoNA Foundation) during the year 2009. The following items were investigated: regional distribution of clinical centers, and type (university or hospital), number of physicians, number of nurses, number of patients followed, antiretroviral drug delivery modality, opening hours of center. Patients enrolled in the ICONA Foundation Study were included into the study. Cumulative mortality rates were estimated using Kaplan-Meier methods, and Cox regression was used to model the effect of prognostic variables on survival.

Results: 2,341 patients were included and 124 death or AIDS-related events were observed in 10,487 patient-years follow-up. Among enrolled patients, $71 \%$ were males, $60 \%>35$-years old, $39.6 \%$ IDU, $37 \% \mathrm{HCV}+, 38 \%$ with pre-cART CD4 cell count $<200 / \mathrm{mm}^{3}$. Concerning clinical center characteristics, $57 \%$ were in the North of Italy, $34.5 \%$ in the Middle and $8.3 \%$ in the South. Median number of individuals followed at each Clinical Center was 1,159 (range 127-4,154) and the median number of patients/per day at each Clinical Center was 34 (range 4-90). Patient-physician relationship was stable in $73.4 \%$ of cases. In only $8.7 \%$ of cases cART was delivered by a pharmacist. In $60.7 \%$ of cases therapy for more than 2 months were given to the patient at each visit. At multivariate analysis, mean number of patient seen per day (IRR 0.99; 95\% CI $0.98-1.00 ; \mathrm{P}=0.04$ ), higher level of CD4 pre-cART (IRR 0.33; $0.21-0.53 ; \mathrm{P}<0.0001$ for the range of CD4 200-350 cells $/ \mathrm{mmc}$, and IRR 0.13 ; 95\% CI 0.09-0.20; P $<0.0001$, when compared with the CD4 stratum 0-200 cells $/ \mathrm{mmc}$ ) and therapy as time-dependent variable (IRR 0.58; $0.38-0.87 ; \mathrm{P}=0.01$ ) were predictors of reduced risk of mortality. Older age $(1.74 ; 1.18-2.55 ; \mathrm{P}=0.005)$, and previous AIDS diagnosis $(2.45 ; 95 \%$ CI $1.06-5.66$; $\mathrm{P}=0.03)$ were significantly associated with increased risk of mortality.

Conclusion: Among different health care delivery characteristics, within an health setting where all clinicians are infectious disease specialists, only the mean number of HIV-infected patients significantly predict mortality, perhaps reflecting both greater physician experience and greater global competency of the clinical center.

\section{CO 42}

SINGLE AND DOUBLE DOSES OF 2009 PANDEMIC INFLUENZA A H1N1 VACCINE ARE EQUALLY IMMUNOGENIC IN HIVINFECTED ADOLESCENTS AND YOUNG ADULTS

A. Vigano ${ }^{*}$, G. Bedogni ${ }^{2}$, E. Giani ${ }^{l}$, V. Giacomet ${ }^{l}$, V. Manfredini ${ }^{l}$, S. Coletto ${ }^{I}$, S. Stucchi ${ }^{1}$, E. Pariani ${ }^{3}$, A. Amendola ${ }^{4}$, G.V. Zuccotti ${ }^{1}$

${ }^{1}$ Pediatric Clinic, L. Sacco Hospital, University of Milan, Milan, Italy; ${ }^{2}$ Clinical Epidemiology Unit, Liver Research Center, Trieste, Italy;

${ }^{3}$ Department of Public Health, Microbiology and Virology, University of Milan, Milan, Italy; ${ }^{4}$ Department of Public Health, Microbiology and Virology, University of Milan, Milan, Italy

Background: Data on the best vaccination schedule for H1N1 pandemic influenza in HIV-infected adolescents and young adults are lacking. We investigated the immunogenicity of 1 (P1) or 2 (P2) doses of pandemic vaccine given at once with seasonal influenza vaccine in this population.

Methods: Seventy-four HIV-infected patients (aging 11-26 years) receiving monovalent MF59-adjuvanted influenza A/California/2009 (H1N1) vaccine containing $7.5 \mu \mathrm{g}$ of hemagglutinin were randomly assigned to $\mathrm{P} 1 \quad(\mathrm{n}=33)$ and $\mathrm{P} 2(\mathrm{n}=31)$. All patients were vaccinated at once with the 2009/10 trivalent seasonal inactivated influenza vaccine. $\mathrm{H} 1 \mathrm{~N} 1, \mathrm{~A} / \mathrm{H} 1 \mathrm{~N} 1, \mathrm{~A} / \mathrm{H} 3 \mathrm{~N} 2$ and $\mathrm{B}$ antigen specific antibody titres (ABT) were analyzed before immunization and 1, 2, 3 and 6 months after. Mixed-effects linear and logistic regression models were used to evaluate protective ABT (dichotomous $>40 \mathrm{U} /$ L) and their changes (continuous and log-transformed) over time. Treatment, time, squared time, and treatment $\times$ time were the fixed effects and the patient was the random effect in these regression models.

Results: P1 and P2 were balanced for sex, age, CD4 T-cells (median 782 vs. 652 cells $/ \mathrm{mL}$ ) and \% of patients with undetectable HIV-RNA (82 vs. 87). Seroprotection and seroconversion for $\mathrm{H} 1 \mathrm{~N} 1$ and the three seasonal strains of the 2009/2010 seasonal vaccine were achieved at 1 month in all patients. As detected by a non-significant treatment $\times$ time interaction, the odds of seroprotection were similar in $\mathrm{P} 1$ and $\mathrm{P} 2$ groups at all time-points for pandemic and seasonal antigens. Thus, $\%$ of $\mathrm{H} 1 \mathrm{~N} 1$ seroprotection were: 3 versus 3 before immunization, 100 versus 100 at 1 months, 100 versus 100 at 2 months, 100 versus 100 at 3 months, 85 versus 97 at 6 months after immunization. Similarly, \% of seroprotection for seasonal antigens were: 24 versus 26 before immunization, 100 versus 97 at 1 months, 100 versus 97 at 2 months, 97 versus 90 at 3 months, 94 versus 83 at 6 months after immunization for $\mathrm{A} / \mathrm{H} 1 \mathrm{~N} 1 ; 18$ versus 16 before immunization, 94 versus 97 at 1 month, 91 versus 90 at 2 months, 75 versus 90 at 3 months, 66 versus 83 at 6 months after immunization for A/H3N2; 9 versus 6 before immunization, 94 versus 97 at 1 month, 82 versus 84 at 2 months, 59 versus 77 at 3 months, 43 versus 40 at 6 months after immunization for B. Likewise, the values of ABT for pandemic and seasonal antigens were similar in P1 and P2 at all time points.

Conclusions: Single and double dose of pandemic A H1N1 vaccine is equally immunogenic in HIV-infected adolescents and young adults. It can be safely coadministered with the 2009/2010 seasonal influenza vaccine.

\section{CO 43}

ARTEMIS: 192-WEEK EFFICACY AND SAFETY OF ONCE DAILY DARUNAVIR/RITONAVIR (DRV/R) VERSUS LOPINAVIR/R (LPV/R) IN TREATMENT-NAÏVE HIV-1-INFECTED ADULTS

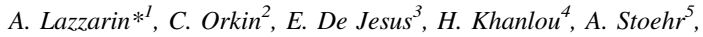
K. Supparatpinyo ${ }^{6}, T$. Van de Casteele $^{7}$, E. Lathouwers ${ }^{7}$, M. Garau ${ }^{8}$ ${ }^{I}$ S. Raffaele Hospital, University of Milan, Milan, Italy; ${ }^{2}$ Barts and The London NHS Trust, London, London, UK; ${ }^{3}$ Orlando Immunology Center, Orlando, Orlando, USA; ${ }^{4}$ AIDS Healthcare Foundation, Los Angeles, Los Angeles, USA; ${ }^{5}$ Institute for Interdisciplinary Medicine, Hamburg, Hamburg, Germany;

${ }^{6}$ Chiang Mai University, Chiang Mai, Chiang Mai, Thailand; ${ }^{7}$ Tibotec BVBA, Beerse, Beerse, Belgium; ${ }^{8}$ Medical affairs Janssen, Milan, Italy

Background: ARTEMIS was a Phase III, randomized, open-label study assessing efficacy and safety of DRV/r 800/100 mg qd versus LPV/r 800/200 mg total daily dose (qd or bid) in treatment-naïve HIV-1-infected adults. At 96 weeks, DRV/r demonstrated non-inferiority and superiority to LPV/r in virological response. Wk 192 results are reported.

Methods: Patients stratified by baseline (BL) viral load (VL [HIV-1 RNA] $\leq 100,000$ copies/mL [cpm]) and CD4 cell count ( $\leq 200$ cells/ $\mathrm{mm}^{3}$ ) were randomized $1: 1$ to DRV/r qd or LPV/r. Primary efficacy parameter: noninferiority $(=-12 \%)$ of $\mathrm{DRV} / \mathrm{r}$ to $\mathrm{LPV} / \mathrm{r}$ in virological response (VL $<50 \mathrm{cpm}$, ITT-TLOVR). DRV/r superiority (=0\%) was assessed if non-inferiority was demonstrated.

Results: 689 patients (30\% female; mean BL VL $4.85 \log _{10} \mathrm{cpm}$; median CD4 225 cells $/ \mathrm{mm}^{3}$ ) were randomised. Overall, significantly more DRV/r than LPV/r patients had VL $<50 \mathrm{cpm}$ at Wk 192, confirming $\mathrm{DRV} / \mathrm{r}$ qd non-inferiority $(\mathrm{p}<0.001)$ and superiority ( $\mathrm{p}=0.002$ ). In patients with virological failure (VF; TLOVR non-VF censored) no developing primary PI mutations were identified in 
either arm; all VFs with paired BL/endpoint phenotypes that were susceptible at BL to amprenavir, atazanavir, indinavir, lopinavir, saquinavir or tipranavir remained susceptible after treatment.

Conclusions: DRV/r qd demonstrated sustained efficacy with non inferiority and superiority to LPV/r over 192 weeks. Development of resistance was low in both arms. DRV/r was associated with smaller median increases in total cholesterol and triglycerides than LPV/r, and a lower incidence of grade $2-4$ diarrhea.

\section{CO 44}

TREATMENT SIMPLIFICATION TO ATAZANAVIR/RITONAVIR PLUS LAMIVUDINE QD IN PATIENTS ON TWO NRTIS PLUS ATAZANAVIR/RITONAVIR WITH OPTIMAL VIROLOGIC CONTROL: 48 WEEKS SAFETY AND EFFICACY RESULTS FROM A PILOT STUDY (ATAZANAVIR AND LAMIVUDINE SIMPLIFICATION STUDY)

A. De Lucal ${ }^{1}$ M. Doino ${ }^{2}$, M. Fabbiani ${ }^{2}$, L. Bracciale ${ }^{2}, N$. Ciccarelli $^{2}$, S. Farina ${ }^{2}$, L. Sidella ${ }^{2}$, A. D'Avino ${ }^{2}$, A. Mondi ${ }^{2}$, R. Murri ${ }^{2}$, R. Cauda ${ }^{2}$, S. Di Giambenedetto $*^{2}$

${ }^{1}$ University Hospital; Second Division of Infectious Diseases, Siena, Italy;

${ }^{2}$ Catholic University, Rome, Italy

Background: ATLAS is a pilot 48 weeks single-arm treatment simplification trial to a two-drug regimen with atazanavir/ritonavir (ATV/ r) + lamivudine (LAM) in HIV-positive patients on a stable atazanavir/ritonavir-based three-drug regimen with HIV-RNA $<50 \mathrm{c} / \mathrm{mL}$. We report safety and efficacy results at 48 -weeks.

Methods: Pts on ATV/r + 2NRTI, without previous treatment failure or resistance to PI or LAM, with HIV-RNA $<50 \mathrm{c} / \mathrm{mL}$ for $>3$ months, CD4 $>200$ cells for $>6$ months, HBsAg-negative and ATV plasma levels >efficacy thresholds were eligible. Study was designed to enroll 40 pts: interruption was planned after five virological failures (VF, confirmed HIV-RNA $>50 \mathrm{c} / \mathrm{mL}$ ). At baseline (BL), regimens were simplified to ATV/r 300/100 mg qd + LAM $300 \mathrm{mg}$ qd. Pts were monitored at $4,12,24,36,48$ weeks. Bone mineral density (BMD), limb fat (DXA), neurocognitive tests, quality of life were analyzed at BL and 48 weeks.

Results: 40 pts enrolled (57\% M, 22\% IDU, 22\% AIDS), with median age 45 years, time since HIV-RNA $<50 \mathrm{c} / \mathrm{mL} 663$ days (IQR 320-895), CD4 count 598 cells/mmc (472-777). 39 pts discontinued tenofovir, 1 abacavir. At analysis, $38 / 40$ patients had reached 48 weeks. According to primary study endpoint, there were 5/38 $(13.2 \%)$ treatment interruption: one death (brain hemorrhage), two reinductions with 2NRTI based on protocol-defined events (1 pregnancy and 1 inadequate ATV concentration) and two VF (both without resistance mutations, undetectable plasma ATV and successfully re-induced with 2NRTI). Median CD4 change was +33 cells $/ \mathrm{mmc}(\mathrm{p}=0.24)$. Six severe adverse events were recorded (4 renal colic, 1 hypertensive crisis, 1 brain hemorrhage). ATV concentration, total bilirubin, LDL cholesterol and triglycerides did not show significant modifications from BL at 48 weeks, whereas significant changes of total cholesterol (mean $+15 \mathrm{mg} / \mathrm{dl} ; \mathrm{p}=0.002)$, HDL $(+6 \mathrm{mg} / \mathrm{dl} ; \mathrm{p}<0.001)$ and GFR $(\mathrm{CG}:+6 \mathrm{~mL} / \mathrm{min}, \mathrm{p}<0.001)$ were observed. Fat was increased in upper $($ mean $+145 \mathrm{~g} ; \mathrm{p}=0.003)$, but not lower limbs and BMD showed a trend towards increase in L2$\mathrm{L} 4\left(+0.01 \mathrm{~g} / \mathrm{cm}^{2} ; \mathrm{p}=0.06\right)$ but not in the proximal femur. There was no significant change in self-reported adherence $(\mathrm{p}=0.85)$ and significant improvement in total physical $(\mathrm{p}=0.002)$ and mental health scores $(p<0.001)$. No change in neuropsychological performance was observed.

Conclusion: Simplification to ATV/r + LAM qd was safe and associated with rare VF, without development of resistance. Further investigation of this strategy in a larger, randomized comparison is warranted.
CO 45

WITH CURRENT ARV TREATMENTS VIROLOGICAL FAILURE MOSTLY OCCURS IN THE CONTEXT OF OPTIMAL SELFREPORTED ADHERENCE

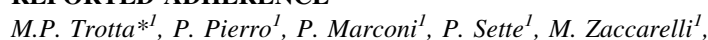

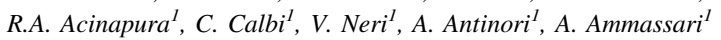
${ }^{I}$ INMI L. Spallanzani, Rome, Italy

Background: It is assumed that ARV adherence $>95 \%$ is sufficient to attain virological suppression. This analysis aims at investigating adherence levels in patients on current ARV treatments, particularly focusing on failing regimen, and at identifying predictive variables of virological failure.

Methods: At clinic visits HIV-infected patients are asked to fill a selfadministered questionnaire to assess ARV adherence: how many times ARV have been taken during the last month [visual analogue scale (VAS)], missed doses in the last week, timing deviation, refill interruption, drug holidays. Demographical and epidemiological characteristics, HIV- and ART-history, CD4 and HIV-RNA are collected from medical charts. Virological failure (VF) was defined as HIV RNA $>50 \mathrm{cp} / \mathrm{ml}$. Distribution of VF percentage across adherence levels within each ARV class has been calculated. In subjects reporting optimal adherence, correlates of VF were assessed by univariate and multivariate logistic analysis.

Results: A total of 3,145 questionnaires were collected from 1,108 patients: F $29 \%$; mean age $45(8.9+\mathrm{SD})$; HIV transmission heterosexual $38 \%$, homosexual 22\%, IVDU $31 \%$; CDC group C $32 \%$; mean CD4 567/mmc (IQR 348-732), HIV-RNA $1.84 \quad$ log $\quad \mathrm{cp} / \mathrm{ml}$ $(0.57+\mathrm{SD})$. Mean time on ARV was 26 months and ART grouping showed NNRTI $=1,050(33.4 \%), \mathrm{PI} / \mathrm{b}=1,842(58.6 \%), \mathrm{PI}=95$ $(3.0 \%)$, INI $=125(4.0 \%)$, CCR5-inhibitor $=39(1.2 \%)$. At VAS mean adherence level was $96.28(\mathrm{SD}+10.6)$ with the following distribution: $\quad<50 \%=29 \quad(1 \%) ; \quad 50-74 \%=100 \quad(3.2 \%)$, $75-84 \%=192(6.2 \%), 85-95 \%=154(5 \%), 96-99 \%=369(12 \%)$, $100 \%=2,250(72.6 \%)$. Virological failure was registered in 586 (18.6\%) patients: $78 \%$ in $>95 \%$ adherence (Fig. 1). Among patients reporting optimal adherence (VAS $>95 \%$ ), independent correlates of VF were: drug holidays (RR 2.93; 95\% CI 2.01-4.29; $\mathrm{P}<0.0001$ ), months of therapy (RR 0.97; 95\% CI 0.96-0.98; P <0.0001), PIboosted (RR 1.55; 95\% CI 0.99-2.43; $\mathrm{P}=0.05$ ), increasing number of ARV regimen (RR 0.96; 95\% CI 0.93-0.99; $\mathrm{P}=0.01$ ).

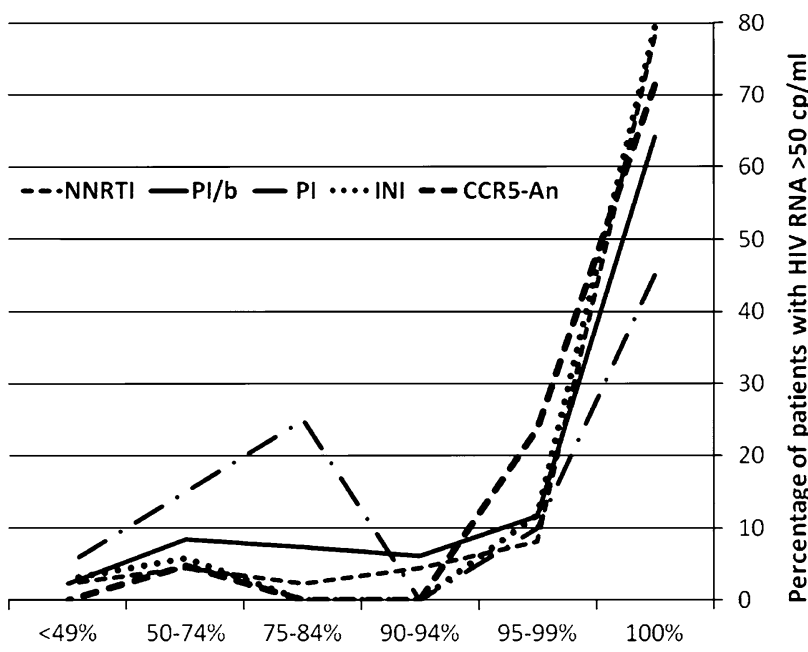

Adherence level

Fig. 1 Self-reported adherence level in patients with HIV RNA $\geq 50 \mathrm{cp} / \mathrm{ml}$ shown by percentage within each ARV group 
Conclusion: With current ARV regimens virological failure mostly occurs in the context of optimal self-reported adherence. In this setting, drug interruption was the main risk factor. A protective role was found for longer duration on ARV and increasing number of ARV regimens, suggesting positive effect of switch to more convenient/ tolerable ARV. Translation into clinical practice may suggest comprehensive adherence assessment, alertness during earlier ARV phase, and ARV tailoring.

\section{CO 47}

FACTORS INFLUENCING LIVER FIBROSIS AND NECROINFLAMMATION IN HIV/HCV COINFECTION AND HCV MONOINFECTION

C. Sagnelli ${ }^{1,2}$, C. Uberti-Foppa ${ }^{l}$, L. Galli ${ }^{l}$, G. Pasquale ${ }^{2}$, S. De Pascalis ${ }^{2}$,

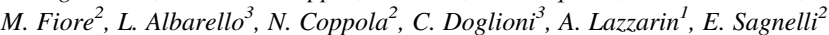
${ }^{I}$ Clinic of Infectious Diseases, Vita-Salute University, San Raffaele Scientific Institute, Milan, Italy; ${ }^{2}$ Department of Public Medicine, Section of Infectious Diseases, Second University of Naples, Naples, Italy; ${ }^{3}$ Department of Pathology, San Raffaele Scientific Institute, Milan, Italy

Aim: To assess differences in liver histology and corresponding causative factors between $\mathrm{HIV} / \mathrm{HCV}$ coinfection and $\mathrm{HCV}$ monoinfection.

Methods: Liver biopsies (LBs) from 440 consecutive HIV/HCV coinfected patients (Group HIV/HCV) and 374 consecutive HCV monoinfected patients (Group HCV) were evaluated for necroinflammation and fibrosis (Ishak) by a pathology unaware of clinical and laboratory data. All patient were naïve for anti HCV treatment, HBsAg negative and with no history of alcohol abuse; $69.3 \%$ of patients in Group HIV/HCV were under an effective HAART at time of liver biopsy.

Results: HIV/HCV coinfected patients compared with those $\mathrm{HCV}$ monoinfected were younger $(38+6$ vs. $46.2+11.6$ years, $\mathrm{p}<0.0001)$, more frequently males $(74.3$ vs. $50.2 \%, \mathrm{p}<0.0001)$ and with HCV-genotype 3 ( 37 vs. $5.8 \%, p<0.0001$ ); they showed a good immunological condition (CD4 $518+266$ ). Patients in Group HIV/ $\mathrm{HCV}$ more frequently than those in Group HCV showed fibrosis score $>4(27.5$ vs. $20.6 \%, p<0.05)$ and necroinflammation score $>9(25.9$ vs. $13.4 \% ; \mathrm{p}<0.0001)$. The prevalence of patients with fibrosis $>4$ increased with the increase of the age both in Group HIV/HCV $(\mathrm{p}<0.005)$ and in Group HCV $(\mathrm{p}<0.05)$; for necroinflammation (score $>9$ ) this increase was found only in Group HIV/HCV $(\mathrm{p}<0.05)$.

Conclusion: Despite an effective HAART, HIV infection favours the progression of liver fibrosis to its more severe forms. Progression of liver fibrosis significantly increases with the increase of the age both in $\mathrm{HIV} / \mathrm{HCV}$ coinfection and in HCV monoinfection, suggesting to consider for an early anti-HCV treatment all patients with HCV related chronic hepatitis.

\section{CO 48}

ASSOCIATION OF KIR/HLA-LIGAND COMBINATIONS WITH DIFFERENT HCV/HIV-RELATED DISORDERS

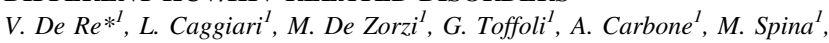
E. Vaccher

${ }^{1}$ Centro di Rifereimento Oncologico, Aviano, Italy

Background and aim: Hepatitis $\mathrm{C}$ virus (HCV) is a pathogen that persists in the presence of a readily apparent immune response. It is associated with hepatitis, cirrhosis, hepatocellular carcinoma (HCC), as well as B-cell lymphoproliferative disorders, as mixed cryoglobulinemia (MC), and B-cell non-Hodgkin's lymphoma (NHL). Moreover, it is noticed an increase in hepatic disease onset in $\mathrm{HCV}+1$ $\mathrm{HIV}+$ coinfected pts with respect to HIV - subjects. Killer immunoglobulin-like receptors (KIRs) genes are polygenic and polymorphic. KIRs were present on T/natural killer (T-NK) cells that constitute a major component of innate immune response. Accumulating evidences indicate that KIR/HLA-ligands play an important role in regulating HCV-related and HIV-related malignancies, by performing innate and adaptive immune response. Aim was to investigate KIR/HLA combinations in different HCV-related malignancies with respect to the same malignancies arising in the context of HIV co-infection to establish the role of KIR/HLA genetic interaction in protection or susceptibility to HCV/HIV-disorders.

Material and methods: We analyzed KIR/HLA genotypes in a selected population of HCV+HBV-HIV- pts with HCC (26 pts), NHL (68), MC (55), chronic HCV infection $(\mathrm{CH}, 29)$. As the second end point we analyzed KIR/HLA genotypes in similar population's but in $\mathrm{HIV}+$ pts; HCC-HIV+ (2 pts), NHL-HIV+ (15 pts), chronic CH$\mathrm{HIV}+(30 \mathrm{pts})$. A group of blood donors (BD, 69) was added as representative of the HCV-HIV- general population. Genomic DNA was genotyped for KIR genes and 2DS4 plus 2DL5 variants by using multiplex polymerase chain reaction-sequence-specific primers (PCRSSP). HLA was genotyped at high resolution by PCR-sequence based typing (SBT) and analyzed with Assign SBT software. For each patient, functional KIR-HLA combinations were determined. Significant differences among groups were estimated by Fisher's exact test and by using multivariate analyses (GraphPad InStat software).

Results: HCC patients without HIV infection were characterized by a less frequency of KIR2DL2 versus $\mathrm{BD} P=0.05$ and $\mathrm{CH} P=0.01$, and of KIR2DL5B and KIR2DS2 genes versus $\mathrm{CH} P=0.01$. Moreover, a lower rate of functional KIR2DL2/C1 and KIR2DS2/C1 combinations were found in $\mathrm{HCC}$ to $\mathrm{CH}, \mathrm{P}=0.05$. By converse, HLA class II DR3-DQ3 clusters were found associated to lymphorpoliferative disorders. Data from HIV+ pts were in course.

Conclusions: Our preliminary findings indicate that there is specific variation of KIR/HLA frequencies in $\mathrm{HCC}-\mathrm{HCV}+$ pts. Results evidence direct $\mathrm{T} / \mathrm{NK}$ functions towards different $\mathrm{HCV}$-related disorders. Moreover, KIR/HLA result genes with a consistently beneficial determinants in the outcome of $\mathrm{HCV}$ infection and consequently protective to $\mathrm{HCC}$ development, while persistence of $\mathrm{HCV}$ is associated to specific KIR/HLA genetic background and lymphoproliferation. The role of KIR/ HLA host background in the setting of HIV-infection would be determined.

\section{CO 49}

HIGHER EXPRESSION OF ADIPONECTIN Q AND LOWER EXPRESSION OF ENHANCER BINDING PROTEIN ALPHA IN ADIPOSE TISSUE CHARACTERIZE HIV-1 INFECTED PATIENTS WITH LIPODYSTROPHY

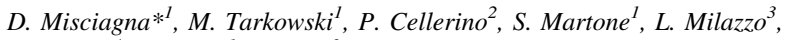
D. Foschi ${ }^{4}$, M. Galli, A. Riva ${ }^{3}$

${ }^{I}$ Dipartimento di Scienze Cliniche, Sezione di Malattie Infettive e Immunopatogenesi, Università di Milano, Milan, Italy; ${ }^{2}$ II Divisione di Chirurgia Generale, Ospedale L. Sacco, Milan, Italy; ${ }^{3}$ III Divisione di Malattie Infettive, Ospedale L. Sacco, Milan, Italy; ${ }^{4}$ Dipartimento di Scienze Cliniche, II Divisione di Chirurgia Generale, Università di Milano, Milan, Italy

Background: Despite the clinical benefits, the long-term antiretroviral therapy is associated with a complex spectrum of untoward metabolic effects, including dyslipidemia and insulin resistance and lipodystrophy syndrome.

Methods: 28 HIV-positive patients attending the Clinic of Infectious Diseases and Tropical Medicine at the University of Milan, with HAART-related lipodystrophy and 11 healthy donors (HC) were enrolled in the study. Subcutaneous adipose tissue specimens from the supra-iliac region were obtained using a 4-mm punch biopsy needle. Fat biopsies were rapidly frozen and stored at $-80^{\circ} \mathrm{C}$. RNA was extracted using standard methods, the cDNA was generated by reverse transcription using transcriptor first strand cDNA synthesis kit (Applied Biosystem) following the manufacturer's instructions. We have compared the mRNA expression profile of adipocytes in the 
HIV-1 positive with lipodystrophy and healthy donors, using TaqMan LDA microfluidic card technology (Applied Biosystem). Statistical analysis was performed using Mann-Whitney test. A written informed consent was obtained from all the participants.

Results: 48 different genes were analyzed ( 47 for the study and one as housekeeping gene). The genes with significantly different expression between the studied groups were Adiponectin Q and enhancer binding protein? (EBP?). Adiponectin is more expressed in HIV-1 positive patients (mRNA expression in arbitrary units (AU): 1.58 in HIV-1 positive versus 0.98 in $\mathrm{HC} ; \mathrm{p}=0.029$ ). EBP? is more expressed in healthy donors (mRNA expression in AU: 2.52 in $\mathrm{HC}$ and 1.54 in HIV-1 positive patients; $p=0.023$ ). In our HIV-1 population we observed a different mRNA expression between females and males in regard to three genes. Adiponectin Q (mRNA expression in AU: 1.05 for female and 1.8 for male $\mathrm{p}=0.011)$, PEPCK-1 (mRNA expression in AU: 2.2 for females and 0.7 for males $p=0.012$ ) and GLUT4 (mRNA expression in AU: 3.3 for females and 1 for males $\mathrm{p}=0.004)$. We also evidenced a different gender specific level of expression for two genes: GLUT4 (mRNA expression in AU 3.13 for females and 2.04 for males $p=0.043$ ) and CSF-1 ( 0.83 for females and 1.07 for males $\mathrm{p}=0.007$ ).

Conclusions: With the limitations of an investigation in a relatively small population, our study suggests a role for CEBP?? and adiponectin in lipoatrophy. In antiretroviral treated HIV infected patients, CEBP? deficiency might lead to defective development of adipose tissue and higher adiponectin might determine increased fatty acid catabolism both leading to lipoatrophy. Differential gene expression in subcutaneous adipose tissue between males and females seems to emerge and might account for the different prevalence and time to appearance of lipodystrophy between sexes.

\section{CO 50}

TDF THERAPY IS INDEPENDENTLY ASSOCIATED TO HYPERPARATHYROIDISM IN A SAMPLE OF 371 HIV INFECTED PATIENTS

D. Pocaterra $*^{l}$, L. Carenzi $^{I}$, M. Schiavini ${ }^{I}$, P. Meraviglia $^{l}$, D. Minisci $^{l}$

M. Bevilacqua ${ }^{2}$, P. Bonfanti ${ }^{3}$, G. Rizzardini ${ }^{l}$

${ }^{1}$ L. Sacco Hospital, Department of Infectious Diseases, Milan, Italy; ${ }^{2}$ L. Sacco Hospital, Endocrinologic Unit, Milan, Italy; ${ }^{3}$ A. Manzoni Hospital, Department of Infectious Diseases, Lecco, Italy

Background: Increased rates of osteopenia and osteoporosis in HIV patients have been associated to highly active antiretroviral therapy (HAART), traditional risk factors and HIV infection itself. A recent report suggested an association between tenofovir (TDF) use and elevated serum levels of parathormone (PTH) whose effect is to stimulate bone resorption in order to normalize calcium serum levels. As hyperparathyroidism may worsen bone mineral loss, we examined the prevalence of secondary hyperparathyroidism and factors associated with HIV patients receiving antiretroviral treatment.

Methods: We performed a cross-sectional analysis considering immunovirological parameters, serum levels of $25-\mathrm{OH}$ vitamin $\mathrm{D}, \mathrm{PTH}$, ionized calcium and HAART treatment in our outpatient clinic for the management of HAART-related toxicity. Patients with primary hyperparathyroidism were excluded from the study. To account for the effects of several factors simultaneously, we used unconditional multiple logistic regression, with maximum likelihood fitting, to obtain odds ratios (OR) and their corresponding 95\% confidence intervals (CI), as estimates of association between hyperparathyroidism and the variables of interest.

Results: 371 patients without primary hyperparathyroidism were included. The mean age was 47 (range 29-75). Males were $57.1 \%$. Hyperparathyroidism (ULN $=65 \mathrm{pg} / \mathrm{ml}$ ) was detected in 65 patients $(17.5 \%)$ and hypovitaminosis D $(<30 \mathrm{ng} / \mathrm{dl})$ was detected in 287 $(77.4 \%)$ patients. $214(57.7 \%)$ subjects were on TDF treatment and $232(62.5 \%)$ on protease inhibitors treatment. The univariate analysis showed a significant direct relation between hyperparathyroidism and age, body mass index (BMI), use of TDF and season (winter/spring vs. summer/fall). An inverse relation emerged with ionized calcium and vitamin D. When all significant variables were included in the multivariate model, an association was confirmed between hyperparathyroidism and TDF use (OR 3.9, CI 1.9-8.0), age (OR 1.5, CI 1.2-1.9), female sex (OR 2.3, CI 1.2-4.5), BMI (OR 1.3, CI 1.1-1.4), season (OR 2.4, CI 1.2-4.9), ionized calcium level (OR 0.16, CI 0.03-0.76) and 25-OH vitamin D (OR 0.7, CI 0.6-0.9).

Conclusions: Our analysis confirms the association between PTH, vitamin D and calcium serum levels. Even though the influence of the high prevalence of hypovitaminosis D $(77.4 \%)$ on PTH through calcium serum levels is evident, an independent effect of TDF use also emerged. The mechanism underlying this association is not clear but the clinical implications could be of great significance.

\section{CO 51}

IS THERE A RELATION BETWEEN MYALGIA AND CENTRAL NERVOUS SYSTEM SYMPTOMS IN PATIENTS RECEIVING RALTEGRAVIR? RESULTS FROM THE SCOLTA PROJECT

G. Madeddu ${ }^{*}$, V. Soddu ${ }^{l}$, E. Ricci ${ }^{2}$, T. Quirino ${ }^{3}$, B. Menzaghi ${ }^{3}$, C. Bellacosa ${ }^{4}$,

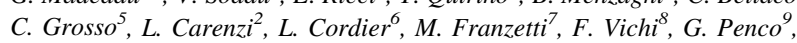
C. Martinelli ${ }^{10}$, P. Maggi ${ }^{4}$, A. Di Biagio ${ }^{17}$, G. Pellicano ${ }^{11}$, L. Corsico ${ }^{12}$, G.V.L. De Socio ${ }^{13}$, E. Mazzotta ${ }^{14}$, G. Parruti ${ }^{14}$, M. Guastavigna ${ }^{15}$, G. Orofino ${ }^{15}$, M.S. Mura ${ }^{1}$, P. Bonfanti ${ }^{16}$

${ }^{1}$ Istituto di Malattie Infettive, Università di Sassari, Sassari, Italy; ${ }^{2}$ I Divisione Malattie Infettive, Ospedali Luigi Sacco, Milan, Italy; ${ }^{3}$ Reparto di Malattie Infettive, Ospedale di Busto Arsizio, Busto Arsizio, Italy; ${ }^{4}$ Unità Operativa di Malattie Infettive, Policlinico di Bari, Bari, Italy; ${ }^{5}$ Reparto di Malattie Infettive, Ospedale Bufalini, Cesena, Italy; ${ }^{6}$ II Divisione Malattie Infettive, Ospedale Luigi Sacco, Milan, Italy; ${ }^{7}$ Clinica Malattie Infettive, Ospedale Luigi Sacco, Milan, Italy; ${ }^{8}$ Unità Operativa di Malattie Infettive, Ospedale Santa Maria Annunziata, Florence, Italy; ${ }^{9}$ Unità Operativa di Malattie Infettive, Ospedale Galliera, Genoa, Italy; ${ }^{10}$ Dipartimento di Malattie Infettive, AOU Careggi, Florence, Italy; ${ }^{11}$ UOC Malattie Infettive, Policlinico G. Martino, Messina; ${ }^{12}$ ASL MB, Servizio MTS, Sesto San Giovanni, Italy; ${ }^{13}$ Clinica Malattie Infettve, Ospedale S.Maria della Misericordia, Perugia, Italy; ${ }^{14}$ Unità Operativa di Malattie Infettive, Ospedale di Pescara, Pescara, Italy; ${ }^{15}$ Divisione A Malattie Infettive, Ospedale Amedeo di Savoia, Turin, Italy; ${ }^{16}$ Struttura Complessa di Malattie Infettive, Ospedale A. Manzoni, Lecco, Italy; ${ }^{17}$ Clnica Malattie Infettive, Ospedale San Martino, Genoa, Italy Introduction: Raltegravir, the first HIV integrase inhibitor available in clinical practice for the treatment of HIV-infection, has shown a good safety profile. However, muscle alterations ranging from asymptomatic creatine phosphokinase (CPK) increases to rhabdomyolysis and central nervous system symptoms (CNS) have been reported in literature. No study to date has evaluated the possible interactions between muscle and CNS adverse events.

Purpose of the study: The purpose of our study was to further investigate the relation between the occurrence of CNS symptoms and muscle alterations in a cohort of patients starting HAART including raltegravir in clinical practice.

Methods: The SCOLTA Project is a prospective, observational, multicenter study created to assess the incidence of adverse events in patients receiving new antiretroviral drugs in clinical practice. Muscle symptoms where classified according to the American Heart Association guidelines a CPK elevations were graded according to the Division of AIDS table for grading the severity of adverse events. The presence of CNS symptoms (headache, dizziness, abnormal dreams, insomnia) were recorded in a standardized form.

Results: A total of $293 \mathrm{HIV}$-infected patients were included in the present study, 188 (64.2\%) males. CDC stage was C in $112(38.2 \%)$ patients. Mean age at enrolment was $44.9 \pm 9.1$ years, mean CD4 cell count $370 \pm 265$ cells $/ \mu \mathrm{L}$ and mean HIV-RNA $3.17 \pm 1.58$ $\log _{10} \mathrm{cp} / \mathrm{ml} ; 106(36.4 \%)$ patients were HCV Ab+ and $119(40.6 \%)$ 
had a diagnosis of lipodystrophy. Fifteen $(5.1 \%)$ patients were naive to antiretrovirals. After a median follow up of 14 months, 261 $(89.1 \%)$ patients were still on therapy with raltegravir. The most frequent causes of discontinuation were patient decision/low adherence in eight (2.8\%), adverse events in four (1.4\%), therapy simplification in four $(1.4 \%)$, death in four $(1.4 \%)$ and virological failure in three $(1.0 \%)$. Overall, $55(18.8 \%)$ patients had muscular events (CPK increases, muscle pain and/or weakness) and $36(12.2 \%)$ reported at least one CNS symptom. No therapy discontinuation was caused by muscle symptoms, CPK elevations or CNS symptoms. Patients with CNS symptoms were significantly $(\mathrm{p}<0.0001)$ more likely to report muscle symptoms $(10 / 17,57.8 \%)$ than those without CNS toxicity $(26 / 276,9.4 \%)$. Furthermore, patients with CNS symptoms had a significantly $(p=0.004)$ higher frequency $(13 / 36$, $36.1 \%$ ) of all muscular events than patients without CNS alterations (42/257, 14.3\%).

Conclusions: Muscle and CNS symptoms occurred frequently in patients receiving raltegravir but were not associated with therapy discontinuation. The occurrence of CNS symptoms appeared to be strongly related with muscle toxicity, in our cases. This observation, even if preliminary, could suggest a higher exposure to raltegravir in such patients, given the high CNS penetration of the drug. Our data suggest that patients with CNS symptoms should be probably monitored more frequently for muscle toxicity.

\section{CO 52}

INCIDENCE TRENDS AND OUTCOME OF NON-AIDS-DEFINING MALIGNANCIES (NADM) IN A COHORT OF HIV-INFECTED PATIENTS DURING THE PERIOD 1985-2008

M. Franzetti ${ }^{\prime}$, F. Adorni ${ }^{2}$, B. Vergani ${ }^{l}$, S. Antinori ${ }^{l}$, M. Galli ${ }^{1}$, A. Ridolfo ${ }^{l}$

${ }^{I}$ Infectious Diseases Unit, Department of Clinical Sciences 'L.Sacco',

University of Milan, Milan, Italy; ${ }^{2}$ Institute of Biomedical Technologies,

National Research Council, Segrate, Milan, Italy

Background: Several studies, based on men predominating cohorts, have reported an increasing incidence of NADM in HIV-positive patients in recent years. Fewer data are available on the spectrum and incidence of NADM in HIV-positive women. The aim of our study was to examine trends in the spectrum, incidence, and survival associated with NADM in a cohort of HIV-positive men and women during periods preceding and following the introduction of HAART. Methods: A retrospective analysis included all incident NADM occurring from 1985 to 2008 in a cohort of 5,920 HIV-positive patients. Spectrum, incidence rates and survival of NADC were compared between pre- (1985-1996) and post- (1997-2008) HAART periods. Incidence rates were examined by Poisson regression models. Standardized incidence ratios compared cancer risk in HIV-infected subjects with that in the general population (Milan Cancer Report). Survival was analyzed by Kaplan-Meier and Cox proportional hazards models.

Results: Among 5,920 patients (4,389 males and 1,541 females) who contributed 46.873 person-years follow up, there were 117 new NADM diagnosis ( 25 in the pre-HAART period and 92 in the postHAART period). The overall incidence rate increased from 1.0 cases/ 1,000 py in the pre-HAART to $4.1 \mathrm{cases} / 1,000$ py in the post-HAART period ( $\mathrm{p}<0.01)$. Hodgkin lymphoma (HL) and lung carcinoma were the most frequent NAMD in both periods (14 and 30 cases, and 6 and 19 cases respectively). A significant expansion in the spectrum of NAMD was observed in the post-HAART period. Of the most common NADM observed in women, risks were higher than expected for cancer of the vulva (SIR, 96.4; 95\% CI 25.9-246.7), ovary (SIR, 6.5; 95\% CI 1.3-18.9), lung (SIR, 6.0; 95\% CI 1.2-17.5), and for HD (SIR, 8.6; 95\% CI 1.0-31.2). In men risks were higher than expected for cancer of anus (SIR, 82.8; 95\% CI 42.7-144.7), liver (SIR, 6.2; 95\% CI 3.2-10.8), tongue (SIR, 5.7; 95\% CI 1.2-16.7), lung (SIR, 2.5; 95\% CI 1.5-4.1), and HL (SIR, 14.3; 95\% CI 9.4-21.0). Five- year survival after NADM diagnosis did not differ between pre- and post-HAART periods (33.6 and $40.7 \%$, respectively; $\mathrm{p}=0.304$ ). No difference in survival was observed according to gender. On the contrary, older age and chronic $\mathrm{HCV}$ infection were significantly associated with mortality, while CD4 cell count at diagnosis (univariate analysis) and antineoplastic treatment were predictive of better survival.

Conclusions: Rates and spectrum of NAMD increased over time within our cohort. Several NAMD, especially those associated with infections and smoking, occurred at rates significantly higher than expected both in men and in women. High mortality rates for NAMD persist in the HAART period and this highlights the need for early diagnosis, use of highly active antiviral treatments and prospective surveillance.

CO 53

HAART IS EFFECTIVE TO PREVENT CERVICO-VAGINAL CYTOLOGICAL ABNORMALITIES RELATED TO HPV HIGHLY ONCOGENIC STRAINS

A. Busto ${ }^{I}$, A. Maddaloni ${ }^{I}$, M.G. Guida ${ }^{\prime}$, M. Sansone ${ }^{2}$, M. D'Abbraccio ${ }^{1}$,

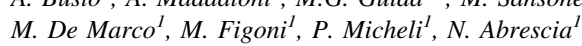

${ }^{1}$ AORN Monaldi-Cotugno-CTO, Naples, Italy; ${ }^{2}$ Università Federico II, Naples, Italy

Background: HIV/HPV coinfected women have an increased risk of developing SIL (squamous intraepithelial lesions) and cervical cancer. The aim of this study is to value the prevalence of HPV highly oncogenic subtypes in a cohort of HIV women from Naples area, to verify if HPV DNA cervical expression is associated with cervical cytological abnormalities and to assess if HAART can reduce the prevalence of HPV related SIL and other cytological abnormalities in cervical tract.

Methods: We collected 170 cervical cytological samples from 170 HIV infected women. On all Pap smears samples a cytological diagnosis was performed. HPV DNA status and oncogenic HPV subtypes were assessed using the Hybrid Capture II $^{\circledR}$. Data regarding HIV RNA plasma levels, CD4+ count and HAART of all patients were analyzed. Statistical evaluations were performed using $\chi^{2}$ test. Results: Median age of 170 enrolled HIV women was 36 years (range 22-55). 50\% were Africans, 38\% Italians, 9\% East Europeans and 3\% South-Americans. Of all 170 vaginal specimens tested, 95 (60\%) were found positive for HPV DNA. Of these 95, 78 (82\%) resulted positive for one or more highly oncogenic HPV subtypes. Only in 17/95 low risk oncogenic subtypes $(\mathrm{p}<0.001)$ were detected. Among 78 women with highly oncogenic subtypes $50(64 \%)$ had CD4 <200/ $\mathrm{mmc}(\mathrm{p}<0.001)$ and $29(37 \%)$ had a HIV-1 RNA plasma viral load $>90,000 \mathrm{cp} / \mathrm{mL}(\mathrm{p}<0.01)$. Of all 170 enrolled patients, $94(55 \%)$ had cytological abnormalities (15 H-Sil, 72 L-Sil, 3 CIN 1, 2 CIN 2-3 and 2 cervical cancers), but only 58/94 (62\%) presented an association with cervical HPV DNA $(\mathrm{p}=0.002)$. Of 94 women with abnormal Pap smears, 45 (48\%) were not in HAART, whereas 49 (52\%) were treated with HAART (65\% PI-based, 30\% NNRTI-based, $5 \%$ other antiretroviral combinations). However, cytological abnormalities associated with highly oncogenic HPV subtypes were found in 12 of $49(24 \%)$ women in HAART and in 25 of $45(60 \%)$ women without HAART $(\mathrm{p}=0.004)$.

Conclusions: Among HIV-HPV coinfected women, highly oncogenic HPV subtypes were strongly prevalent $(82 \%)$. Cervical cytological abnormalities were associated both with cervical HPV DNA and with severe immunodeficiency. High plasma HIV-1 viral load was not associated with cervical expression of highly oncogenic HPV strains. Finally, our data demonstrated a protective role of HAART to prevent cervico-vaginal cytological abnormalities, including cervical cancer, related to HPV highly oncogenic strains 


\section{CO 54}

PREVALENCE OF HPV SUBTYPES AND ANAL DYSPLASIA AMONG A GROUP OF HIV-POSITIVE PEOPLE WITH ANAL COMPLAINS

I. Dal Conte ${ }^{*}$, M. Mistrangelo ${ }^{2}$, V. Ghisetti ${ }^{3}$, P. Cassoni ${ }^{4}$, M.G. Milia ${ }^{3}$, M.L. Stella $^{1}$, G. Gregori ${ }^{3}$, M. Morino ${ }^{2}$, G. Di Perri ${ }^{5}$

${ }^{I}$ STI Clinic-Amedeo di Savoia Hospital-ASLTO2, Turin, Italy; ${ }^{2}$ Department of Digestive and Colorectal Surgery, Centre of Minimal Invasive Surgery, San Giovanni Battista Hospital, University of Turin, Turin, Italy; ${ }^{3}$ Microbiology and Virology Laboratory, Amedeo di Savoia Hospital, ASL TO2, Turin, Italy; ${ }^{4}$ Department of Biomedical Sciences and Human Oncology, San Giovanni Battista Hospital, University of Turin, Turin, Italy; ${ }^{5}$ Infectious Diseases Department, Amedeo di Savoia Hospital, University of Turin, Turin, Italy

Background: Infection with high risk human papillomavirus (HPV) is a necessary condition for the development of dysplastic epithelial lesions; several studies have assessed a strong correlation between HPV infection and anal cancer. In HIV-positive homosexual men the rate of anal cancer is almost double than in seronegative ones (more than 70 cases per 100,000 persons-year). Studies of a cohort of HIVseronegative and -seropositive homosexual men have shown a 60 and 93\% prevalence of anal HPV infection, respectively, with a higher proportion of multiple infections in HIV-positive men (73 vs. $23 \%$ ) Objectives: To study the prevalence of HPV infections in a group of HIV-positive people with anal complains; to evaluate the distribution of different HPV strains and correlate the prevalence of high-risk strains with cell degeneration. Subjects and methods: a group of consecutive male and female patients referred to the Coloproctology Service of STI Clinic, Amedeo di Savoia Hospital in Turin underwent anoscopic examination. The presence of HPV was evaluated collecting anal swabs that were analyzed using INNO-LiPA HPV Genotyping Extra (Innogenetics, B) a line probe assay (LIPA), based on the reverse hybridization principle, designed for the identification of 28 different HPV genotypes by the detection of specific sequences in the $\mathrm{L} 1$ region of HPV genome.

Results: A total 31 persons have been studied ( 7 females and 21 males), the median age was 45 years (range 27-64). All the women and $25 \%$ of men were heterosexual. The average number of lifetime partners was over 50 . A previous STI was reported by $45 \%$ of subjects. In 25 out of 33 cases a good cytological sample was obtained: $19 / 25(76 \%)$ were normal; $8 \%(2 / 25)$, had an inflammatory picture; $8 \%(2 / 25)$ were compatible with AIN III; $8 \%(2 / 25)$ were compatible with ASCUS. In 25/25 cases HPV genome was detected. In two cases the LIPA typing was unable to identify a particular HPV genotype; in $6 / 25(24 \%)$ a low risk strain was found, in $2 / 25(8 \%)$ a high risk strain was detected and $56 \%(14 / 25)$ had a mixed infection with strains of high and low risk. HPV16 was the prevalent strain, followed by HPV18 (32\%). The most clinical significant aspect was the finding of carcinoma of the anus (two multifocal) in three cases out of $31(10 \%)$. These were all symptomatic patients with HPV infection, but only one with a positive cytology (AIN III- HPV16 and 18).

Conclusion: Regular anoscopy is an essential step for the management of HIV-positive people and this practice cannot be replaced by cytological examination only. Molecular tests confirm the high prevalence of HPV infection in HIV-infected patients with a history of anal sexual exposure.

\section{SC 01 \\ HIVOICES \\ F. Porcari*1, E. Pullega ${ }^{l}$, S. Mattioli ${ }^{1}$ \\ ${ }^{1}$ Arcigay, Bologna, Italy}

Background: We attempted to verify the existence of a connection between internalized homophobia and the acceptance of one's own HIV status in HIV infected men who have sex with men (MSM).
Methods: Arcigay Bologna's Cassero Salute, with contributions from Arcigay Nazionale and Nadir Onlus, produced a 3-day residential experiential training group, set in a protected environment and aimed at a group of 26 participants aged between 24 and 57, homosexual-bisexual, HIV positive biologically male. The two trainers (Emanuele Pullega and Filippo Porcari) and an observer, proposed a series of structured and unstructured activities, which used a combination of a bodily, non-verbal and emotional language that may encourage interaction and sharing of one another's personal background in the context of the following themes: sense of belonging, sexual identity, internalized homophobia and the visibility of one's own HIV status. At the end of the training experience the participants filled in an evaluation form.

Results: All the participants declared that their self-esteem had increased. $88 \%$ of them stated that they had acquired new tools useful to better live with their HIV status. All the participants claimed to have experienced new ways to better appreciate their own emotions. $85 \%$ of them found analogies between the process of acceptance of their own sexual orientation and their HIV positive status. All the participants noted increased capacities in recognizing the fears connected to their HIV status.

Conclusions: The most noticeable result is the extraordinary speed with which the participants felt they belonged to the group and identified with it. This need to belong highlights how the target subjects are considered outcast even within the LGBTQ community itself. We have scheduled two new experiential training groups for 2011, with the goal of acquiring new data to verify the hypothesis at the core of the work, following the desire of the participants and on the basis of the need for knowledge and exchange made evident by the group.

\section{SC 02}

GAY SEX ON THE WEB IN MILAN: SOCIAL NEEDS AND RISKY BEHAVIORS

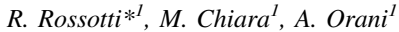

${ }^{I}$ SC Malattie Infettive e Tropicali, AO “A. Manzoni”, Lecco, Italy

Introduction: The Web nowadays represents an important tool for communication; Social Networks and chat-rooms are often a virtual meeting point to seek occasional sexual partners. They have been investigated to assess if sexual intercourses starting online are more risky than traditional encounters. Existing literature shows contrasting data for either heterosexual and men who have sex with men (MSM) sex seekers; these works have been conducted in Anglo-Saxon countries while Southern Europe has never been investigated. Aim of our study is to analyze users from a popular gay chat-room in an area with a high HIV prevalence such as Milan to evaluate their risk perception and sexual behavior.

Methods: Gayromeo.com is one of the most popular gay chat-room where users can create a personal profile to describe themselves, demographic and social features, social and sexual needs and habits. New users from Milan who created a profile from December, 23 to December, 302010 were included in the study. Users looking for sexual partners were further analyzed to evaluate condom use and factor associated with risky behaviors. Statistic analyses were performed with descriptive and logistic regression approaches.

Results: During the study period an average of 59,953 users were available on the website; 682 were new users from Milan who recently created a personal profile. Their socio-demographic features are summarized in Table 1. Of note, $33.3 \%$ were online looking for friendship and/or committed relationships; the remaining $66.7 \%$ $(\mathrm{N}=455)$ were sex seekers. The majority $(65.5 \%)$ claimed to perform only safe sex, while the remaining did not (bareback $0.7 \%$; "needs discussion" 8.3\%; "not declared" 25.5\%). Risk factors associated with unsafe sex are shown in Table 2.

Discussion: Users of this popular gay chat-room often enter it not for sexual encounters but just for other kind of relationships. HIV-positive users are a minority, but there is probably an underreporting 
problem; those who declare to be positive are often online not for sex but for friends. Among sex seekers, the majority state to perform only safe sex, while the others choose to be reticent to gain greater bargaining power and appeal: being reserved about his own status seems a risk factor to engage in unsafe sexual intercourse. Condom use has still cultural implications among MSM and uncertainty about its use gives more power during the online bargain. The communicative power of these reserves is so strong that users at higher risk do not even declare to look for anal sex, since it is probably implied in their sexual messages. On the other hand, younger users ( $=20$ years of age) and anal receptive sexual partners are at higher risk to engage in unsafe practices. These data underline the need to strengthen educative efforts for MSM for a more conscious condom use.

Table 1 Socio-demographic features of study population

\begin{tabular}{|c|c|}
\hline \multicolumn{2}{|l|}{ Age (years; $N=682$ ) } \\
\hline Average & 30.2 \\
\hline Median \pm SD & $29 \pm 8.5$ \\
\hline \multicolumn{2}{|l|}{ Ethnicity $(\% ; \mathrm{N}=578)$} \\
\hline Caucasian & 69.6 \\
\hline Mediterranean & 18.5 \\
\hline Latin & 6.0 \\
\hline Mixed & 2.3 \\
\hline Asian & 2.6 \\
\hline Arab & 0.5 \\
\hline Black & 0.5 \\
\hline \multicolumn{2}{|l|}{ Occupation $(\% ; \mathrm{N}=84)$} \\
\hline Employee & 35.7 \\
\hline Student & 40.5 \\
\hline Self-employed & 19.0 \\
\hline Unemployed & 4.8 \\
\hline \multicolumn{2}{|l|}{ Religion $(\% ; \mathrm{N}=60)$} \\
\hline Christian & 31.7 \\
\hline New Ager & 5.0 \\
\hline Buddhism & 3.3 \\
\hline Agnostic/atheist & 60.0 \\
\hline Spoken languages (mode) & 2 \\
\hline Smoker $(\% ; N=473)$ & 46.1 \\
\hline \multicolumn{2}{|l|}{ Relationship status $(\% ; \mathrm{N}=483$ ) } \\
\hline Single & 83.2 \\
\hline Open relationship & 4.6 \\
\hline Committed relationship & 9.3 \\
\hline Married & 2.9 \\
\hline HIV-positive (\%; $\mathrm{N}=682)$ & 2.6 \\
\hline Sex seeker $(\% ; N=682)$ & 66.7 \\
\hline HIV-positive sex seekers $(\% ; \mathrm{N}=682)$ & 1.0 \\
\hline
\end{tabular}

Table 2 Factors associated with a risky sexual online behaviour (logistic regression analysis)

\begin{tabular}{llll}
\hline Risk factor & OR & $95 \%$ CI & $\mathrm{p}$ \\
\hline $\begin{array}{l}\text { Age (years) } \\
18-20\end{array}$ & $\mathbf{2 . 6 6}$ & $1.32-5.35$ & $\mathbf{0 . 0 0 5}$ \\
\hline
\end{tabular}

Table 2 continued

\begin{tabular}{|c|c|c|c|}
\hline Risk factor & OR & $95 \% \mathrm{CI}$ & $\mathrm{p}$ \\
\hline $21-25$ & 1.17 & $0.73-1.88$ & $>0.05$ \\
\hline $26-30$ & 1.04 & $0.66-1.63$ & $>0.05$ \\
\hline $31-35$ & 0.77 & $0.47-1.28$ & $>0.05$ \\
\hline $36-40$ & 0.81 & $0.45-1.47$ & $>0.05$ \\
\hline $41-45$ & 0.61 & $0.27-1.40$ & $>0.05$ \\
\hline $46-50$ & 1.45 & $0.60-3.52$ & $>0.05$ \\
\hline$>50$ & 0.95 & $0.17-5.24$ & $>0.05$ \\
\hline \multicolumn{4}{|l|}{ Ethnicity } \\
\hline European & 0.94 & $0.48-1.86$ & $>0.05$ \\
\hline Non European & 1.06 & $0.54-2.09$ & $>0.05$ \\
\hline Reserve about his own ethnicity & 2.27 & $1.28-4.03$ & 0.004 \\
\hline \multicolumn{4}{|l|}{ Relationship } \\
\hline Single & 1.02 & $0.57-1.83$ & $>0.05$ \\
\hline Open relationship & 1.55 & $0.57-4.18$ & $>0.05$ \\
\hline Committed relationship & 0.67 & $0.29-1.53$ & $>0.05$ \\
\hline Married & 1.37 & $0.44-4.28$ & $>0.05$ \\
\hline Reserve about his own relationship & 1.69 & $1.09-2.64$ & 0.019 \\
\hline \multicolumn{4}{|l|}{ HIV-status } \\
\hline Seropositivity & 1.43 & $0.32-6.48$ & $>0.05$ \\
\hline \multicolumn{4}{|l|}{ Sexual role } \\
\hline Insertive & 0.66 & $0.36-1.18$ & $>0.05$ \\
\hline Receptive & 1.83 & $1.14-2.95$ & 0.012 \\
\hline Versatile & 0.73 & $0.46-1.17$ & $>0.05$ \\
\hline Reserve about his own sexual role & 8.66 & $4.88-15.16$ & $<\mathbf{0 . 0 0 1}$ \\
\hline \multicolumn{4}{|l|}{ Sexual practice } \\
\hline Anal sex & 0.05 & $0.02-0.11$ & $<\mathbf{0 . 0 0 1}$ \\
\hline Fisting & 1.05 & $0.60-1.84$ & $>0.05$ \\
\hline Sadomasochism & 0.86 & $0.55-1.34$ & $>0.05$ \\
\hline Dirty & 0.75 & $0.46-1.22$ & $>0.05$ \\
\hline Fetish & 0.55 & $0.37-0.82$ & 0.003 \\
\hline
\end{tabular}

SC 03

THE GIOCASICURO PROJECT: RESULTS OF A COMPREHENSIVE INTERVENTION FOR THE PREVENTION AND DIAGNOSIS OF SEXUALLY TRANSMITTED INFECTIONS AMONG MEN WHO HAVE SEX WITH MEN IN ITALY

I. Dal Conte $*^{1}$, V. Cucco $^{4}$, G. Salamina ${ }^{2}$, A. Zambon ${ }^{3}$, Members of Giocasicuro Project ${ }^{5}$

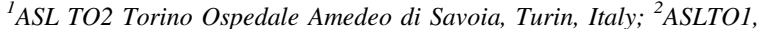

Dipartimento di Prevenzione, Turin, Italy, ${ }^{3}$ Università degli Studi, Turin, Italy; ${ }^{4}$ Coordinamento Torino Pride LGBT, Turin, Italy; ${ }^{5}$ Giocasicuro, Turin, Italy

Background: The incidence of sexually transmitted infections (STIs) is on the rise worldwide. Among new cases of STIs, treponemal infections are prevalently diagnosed in men who have sex with men (MSM). STIs play a known role in the spread of HIV; however, the scarce cooperation between public health care agencies and community-based associations in Italy has meant that most prevention projects are top-down interventions. Moreover, the lack of a longterm national HIV prevention campaign and a sort of false optimism about HIV disease, engendered by readily available pharmacological 
treatment, have lowered risk perception and subsequently raised barriers to adopting preventive behaviours by susceptible and infected persons alike.

Objectives: To implement a syphilis/HIV control program targeting MSM according to best health promotion guidelines through a collaborative project carried out by the Italian national health service (NHS) and LGBT associations.

Methods: A multidisciplinary group was formed to devise a multistep primary prevention intervention addressing MSM in three metropolitan areas: Turin, Rome, and Milan. The program, entitled "Giocasicuro Project", was carried out in collaboration with Coordinamento Torino Pride LGBT.

Results: In July 2007, the project was launched in Turin. Seven health promoters recruited through local LGBT associations received training: (1) to improve through individual and collective counselling knowledge about STIs and the risks associated with unprotected sex, and how to sustain behavioural changes; (2) to promote condom use; and (3) to promote access to STIs clinics. By joint agreement between an NHS-operated STI clinic and the owners of gay bars, saunas and other venues, the promoters designed sexual health interventions, featuring a variety of activities depending on venue clientele: individual counselling and testing, discussion groups, meetings, focus groups, themed shows. Printed educational materials $(6,000$ cards, 50 posters, 115,000 brochures, 10,000 flyers), videos, web tools, and 65,000 safer-sex kits (2 condoms and 1 lubricant inside; safe-sex rules on the kit package) were created and distributed over the following 2 years in the three metropolitan areas and shortly thereafter by 46 provincial committees headed by Arcigay.

Conclusion: This is the first national health project designed to stem the resurgence of STIs/HIV among MSM in Italy; the innovative feature is that it was conducted jointly by public health agencies and LGBT associations. This approach might be taken as a benchmark for implementing a global national strategy for HIV/ STIs control.

(This project was supported by a grant $\mathrm{CCM}-$ Ministry of HealthItaly).

\section{SC 04 \\ FACILITATED ACCESS PROCEDURES FOR HIV AND HCV TESTING IN VULNERABLE GROUPS \\ M.L. Cosmaro ${ }^{* 1}$, M. Oldrini ${ }^{1}$, L. Rancilio ${ }^{2}$, A.M. Villa ${ }^{3}$, M.R. Parisi ${ }^{4}$,

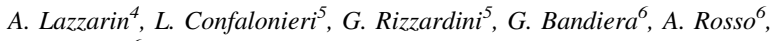 A. Morrone 6 \\ ${ }^{1}$ LILA Milano ONLUS, Milan, Italy; ${ }^{2}$ CARITAS Ambrosiana, Milan, Italy; ${ }^{3}$ Ambulatorio Opera San Francesco per i poveri, Milan, Italy; ${ }^{4}$ Department of Infectious Disease-IRCCS San Raffaele Scientific Institute, Milan, Italy; \\ ${ }^{5}$ Department of Infectious Diseases, Luigi Sacco Hospital, Milan, Italy; ${ }^{6}$ NIHMP National Institute for Health, Migration and Poverty, Rome, Italy}

During the Social and clinical study aimed at facilitating prevention, diagnosis and follow-up of HIV/AIDS infections and coinfections in socially and economically disadvantaged groups carried out by NIHMP and CAA in 2009-2010, the persisting difficulties of vulnerable populations in accessing HIV screening centres became evident, as well as the need to experiment a different approach. In Milan, despite a network of private NGOs actively promoting HIV testing in their dedicated services and two public screening units (CRH ASL Milano and Infectious Diseases Division H. L.Sacco) ensuring facilitated access for vulnerable people (migrants, IDU, SW, homeless), the resulting impact was poor and led to the decision to assess feasibility of proposing rapid HIV/HCV tests to vulnerable populations in lowthreshold services (clinic Opera San Francesco, OSF, for migrants and
Drop-In Centre for drug/alcohol users) and to verify the response of vulnerable populations to this approach.

Methodology and planned activities: Definition of protocols with clinic OSF and Drop-In Centre, for the promotion and offer of rapid HIV1-2 and HCV antibody tests to their clients; definition of protocols with H. San Raffaele and H. L.Sacco for confirmation tests on blood in case of preliminary positive results. Definition of procedures for pre-test counselling, informed consent form and collection of patient's agreement, testing of patients on oral fluid, communication of results, post-test counselling. Specific training of counsellors and medical staff in pre-post test counselling and correct use of OraQuick Advance Tests. Preparation of forms for data collection.

Results: In November-December 20105 days were devoted to experiment the new approach ( 3 at OSF, 2 at DropIn). In 3 set dates 442 patients accessed OSF clinic. They were all asked to undertake HIV/HCV counselling: 166 accepted (37.6\%). In 17/166 cases decision was taken not to proceed due to language problems and one person decided not to test. Of 74 clients accessing the Drop-In in 2 days, $33(44.6 \%)$ accepted to receive pre-test counselling and all of them were tested. Overall 181 people were tested for HIV and 169 also for HCV. 116/181 were males; only 7/181 were Italian. In 180 cases the HIV test was negative; one person had an unclear result and was addressed to hospital for confirmation; she resulted negative. 133/181 people had never tested for HIV before. $69.7 \%$ referred risk, $91.2 \%$ of them to unprotected sex. Two HCV tests resulted positive; both patients knew they had hepatitis but could not remember which type. During counselling patients declared to prefer the less invasive procedure of testing the oral fluid and the short waiting time prior to result communication $\left(20^{\prime}\right)$. The staff of OFS and Drop-In expressed satisfaction for outcomes of initiative.

Conclusions: Study on new approach demonstrated the possibility of testing vulnerable populations in low threshold services and the positive response of such groups to it.

\section{$\mathrm{SC} 05$}

TEST DAY IN THE ERA OF "SEEK, TEST AND TREAT" FOR HIV

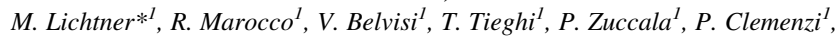
L. Di Giacomo ${ }^{2}$, V. Mercurio $^{2}$, G. Colazingari ${ }^{2}$, C.M. Mastroianni ${ }^{1}$ ${ }^{1}$ Sapienza Universita di Roma, Latina, Roma; ${ }^{2}$ Ospedale SM Goretti, Latina, Italy

Introduction: In the era of test and treat HIV, we believe that universal testing are fundamental to improve early diagnosis and to avoid unaware HIV transmission and AIDS presenter patients. However many factors obstacle universal testing showing socio-economical reasons. In our setting we have a low testing activity (about 500 tests per year) with a high rate of HIV diagnosis per year (80\% of AIDS presenters). At our center HIV test is offered as anonymous and free blood test with pre and post counseling.

Methods: An open door event test day was set up for the World AIDS day (1 December) with $12 \mathrm{~h}$ opening access from 7.30 a.m. to 7.30 p.m.). The event was organized with the medical and nurse staff of our center and the cooperation of the local ONG LILA and Sapienza University students of Medicine Faculty of Latina. Precounseling was performed following HIV guidelines by medical staff. A question list was performed to collect socio-demographic characteristics and sexual behaviors. HIV-test was performed on sera samples by ELISA Combo (Abbot).

Results: 165 subjects adhered to the HIV-test day with a higher prevalence of female sex $(60 \%)$. The median age was 25 years with a broad spectrum between 22 and $68.65 \%$ of subjects did HIV test for the first time in their life. Most subjects revealed sexual relation with 
potential risk for HIV infection. The majority (94\%) of persons declared heterosexual relations, only $1 \%$ was MSM. Only $31 \%$ used condom in their sexual intercourse-HIV test was negative for all subjects. Comparing this data with the usual population tested in our center we noticed that during "open day event" we target a population of never testers with an higher proportion of women showing sexual relation without barrier methods.

Conclusions: Emerging data showed the importance of integrated action to fight HIV that involve both primary and secondary prevention with ART treatment. Expanded voluntary HIV testing seems to be important not only in HIV high burden country, but also in our settings to target subjects only apparently at low risk for HIV infection empowering them to take control of their health by seeking medical care and adopting behaviors to prevent HIV transmission.

\section{SC 06}

ACUTE CASE DEFINITION FOR BEDSIDE DIAGNOSIS OF SUSPECTED/PROBABLE HIV-1 INFECTION IN THE COMMUNITY SETTING

M. Tavio ${ }^{\prime}$, R. Del Gobbo ${ }^{l}$, A. Mataloni Paggi ${ }^{l}$, A. Riva ${ }^{l}$, F. Burzacchini ${ }^{l}$, M. Simeone ${ }^{l}$, L. Scudeller ${ }^{l}$

${ }^{I}$ Divisione di Malattie Infettive, Ospedali Riuniti di Ancona, Ancona, Italy

Acute and chronic HIV-1 infection can be considered as two clinical entities sharing the same etiology, but deserving distinctive diagnostic approaches. During the acute phase, indeed, the clinical picture is entirely and directly amenable to HIV-1 activity, but signs and symptoms are utterly unspecific and diagnosis is difficult without a high index of suspicion [1]. Beginning the chronic phase instead, HIV-specific symptomatology essentially eclipses, and the disease is usually diagnosed by the presence of other affections directly related to the underlying immunosuppression like tumors and other infections. Owing to the natural history of HIV-1 disease, case definitions over time principally focused on expanding the list of AIDS-defining events in the chronic phase, rather than setting specific approaches towards clinical HIV-1 diagnosis during the acute phase. In particular, a specific case definition of acute HIV-1 infection to the purpose of surveillance and control has never been released, not even in the most recent guidelines [2]. In US it has been estimated that every 9 min a new HIV-1 infection occurs [3]; since acute infection frequently results in symptomatic disease [4], a significant proportion of these patients is likely to seek medical advice, without obtaining in many cases adequate counseling for HIV-1: in fact, in US up to $45 \%$ of patients receive an AIDS diagnosis within 3 years after receiving their initial HIV-1 infection diagnosis, that is years after acute infection has been established [5]. It is universally accepted that lack of identification of acute HIV-1 infection has extremely severe consequences involving both individuals and community: antiretroviral therapy is not offered at the optimal time for the patient, and HIV-1 transmission is not impeded during the phase of maximal infectivity, due to the patient's unawareness of infection and transmissibility, the likely persistence of risk behaviour, and the very high HIV-1 concentration in plasma and semen. We suggest that by means of active surveillance of acute HIV-1 infection we might be able to reduce HIV-1 transmission. The efficacy of this approach has been shown in other severe acute viral infections epidemics (e.g. SARS). We propose the development of a "bedside" acute case definition based on a combination of clinical and epidemiological criteria like those presented in Table 1, to the purpose to enhance visibility of HIV-1 acute presentation in the community medicine; such definition is meant to stratify patients at the bedside (suspect/probable acute cases of HIV-1 infection), to be then lab-referred for confirmation. This definition should ideally be combined to an educational campaign to the wide lay public and health care practitioners alike (particularly at the primary care level), to actively search for cases and improve sensitivity. Finally, it should be validated on the field.

Table 1 Case definitions for community bedside diagnosis of acute HIV-1 infection

\begin{tabular}{|c|c|c|c|}
\hline Clinical criteria & $\begin{array}{l}\text { Epidemiological } \\
\text { criteria }\end{array}$ & $\begin{array}{l}\text { Provisional } \\
\text { diagnosis }\end{array}$ & Confirmed case \\
\hline \multirow[t]{2}{*}{$\begin{array}{l}\text { A person } \\
\text { presenting } \\
\text { with history of } \\
\text { signs and } \\
\text { symptoms } \\
\text { suggestive of } \\
\text { an acute viral } \\
\text { infection }^{\text {a and }}\end{array}$} & $\begin{array}{l}\text { A 6-months } \\
\text { history of } \\
\text { possible } \\
\text { exposure to } \\
\text { HIV-1 } \\
\text { infection } \\
\text { through } \\
\text { susceptible } \\
\text { contacts with } \\
\text { a source of } \\
\text { unknown } \\
\text { HIV-1 status }\end{array}$ & $\begin{array}{l}\text { Suspected } \\
\text { case }\end{array}$ & $\begin{array}{l}\text { Any suspect or } \\
\text { probable case } \\
\text { with positive } \\
\text { results to } \\
\text { laboratory } \\
\text { criteria }\end{array}$ \\
\hline & $\begin{array}{l}\text { A 6-months } \\
\text { history of } \\
\text { certain } \\
\text { exposure to } \\
\text { HIV-1 } \\
\text { infection } \\
\text { through } \\
\text { susceptible } \\
\text { contacts with } \\
\text { an HIV-1- } \\
\text { infected } \\
\text { source }^{\mathrm{b}}\end{array}$ & $\begin{array}{l}\text { Probable } \\
\text { case }\end{array}$ & \\
\hline $\begin{array}{l}\text { Laboratory } \\
\text { criteria }\end{array}$ & \multicolumn{3}{|c|}{$\begin{array}{l}\text { HIV-1 testing (HIV-1 antibodies tests, with or } \\
\text { without plasma HIV-1-RNA viral load) should } \\
\text { be offered to any suspect or probable case, and } \\
\text { those with appropriate positive results will be } \\
\text { reclassified as confirmed cases. }\end{array}$} \\
\hline $\begin{array}{c}\text { Exclusion } \\
\text { criteria }\end{array}$ & \multicolumn{3}{|c|}{$\begin{array}{l}\text { A case should be excluded if an alternative } \\
\text { diagnosis can fully explain the illness. }\end{array}$} \\
\hline
\end{tabular}

${ }^{\text {a }}$ Fever $\left(>38^{\circ} \mathrm{C}\right)$, AND one or more of the following signs and symptoms: fatigue; pharyngitis; myalgia or arthralgia; nausea or vomiting; diarrhoea; headache; rash; lymphadenopathy; weight loss; diaphoresis or night sweats; oral or genital ulcers; aseptic meningitis ${ }^{1,4}$

b One or more of the following types of exposure during the 6 months prior to onset of symptoms: unsafe sex; needle exchange; any other meaningful exposure to high risk biologic fluid because of occupational or non-occupational reasons

\section{SC 07 \\ EFFICACY AND SAFETY OF ONCE DAILY MARAVIROC PLUS LOPINAVIR/RITONAVIR IN ANTIRETROVIRAL NAÏVE HIV- INFECTED PATIENTS \\ S. Nozza ${ }^{* 1}$, A. Antinori ${ }^{2}$, F. Mazzotta ${ }^{3}$, C. Calbi ${ }^{2}$, M. Di Pietro ${ }^{3}$, L. Galli ${ }^{1}$, C. Tommasi ${ }^{2}$, R. Fezza ${ }^{2}$, P. Narciso ${ }^{2}$, G. Tambussi ${ }^{1}$, A. Lazzarin ${ }^{1}$ \\ ${ }^{I}$ San Raffaele Scientific Institute, Milan, Italy; ${ }^{2}$ INMI L. Spallanzani, Rome, Italy; ${ }^{3}$ Ospedale S.M. Annunziata, Florence, Italy}

Background: Current guidelines recommend three drug combinations to treat antiretroviral naïve HIV-infected patients; some data of novel strategies with NRTI-sparing regimen in this setting are now available. The study compares immunovirological efficacy and safety 
of once daily maraviroc (MVC) $150 \mathrm{mg}$ plus lopinavir/ritonavir (LPV/r) to tenofovir/emtricitabine (TDF/FTC) plus LPV/r.

Methods: This is an ongoing, proof-of-concept, randomized, openlabel, 48 weeks trial. Data were collected at baseline (BL) and at 4, 12, 24, 36 and 48 weeks. Comparisons between groups evaluated by the Chi-square or Mann-Whitney rank-sum test. Results reported as median (Q1-Q3) or frequency $(\%)$, as appropriate.

Results: Up to date, 41 pts (20 MVC, 21 TDF/FTC) were enrolled: age 40 years, $1 / 41$ female, infected since 3.8 years. At BL:median CD4 285 cells/ $\mu \mathrm{L}$; CD4\% 18.5, HIV-RNA 4.4 (3.9-4.9) $\log _{10}$. No difference in $\mathrm{BL}$ characteristics were found between the two treatment groups. At W24, all patients in both groups had HIV-RNA $<50$ copies/ml; CD4 cells count increased in both groups. HIVRNA decrease and CD4 cells count increase was more rapid in MVC group. Immunological and virological results are showed in Table 1. Treatment was well tolerated, without significant increase in AST, ALT, CPK, cholesterol, triglycerides and glycaemia; 3/41 (7\%) pts discontinued the study due to diarrhea LPV/r.

Table 1 Immunological and virological results

\begin{tabular}{clllll}
\hline \multicolumn{1}{c}{ BL } & W4 & W12 & W24 & W36 \\
\hline $\begin{array}{c}\text { CD4 (cells/ } \\
\mu \mathrm{L})\end{array}$ & MVC: & MVC: & MVC: & MVC: & MVC: \\
& 277 & 444 & 469 & 524 & $533(355-$ \\
& $(250-566)$ & $(216-644)$ & $(216-701)$ & $(290-688)$ & TDF/FTC: \\
& TDF/FTC: & TDF/FTC: & TDF/FTC: & TDF/FTC: & 482 \\
& 293 & 403 & 468 & 496 & $(350-616)$ \\
& $(234-445)$ & $(229-341)$ & $(224-579)$ & $(241594)$ & \\
CD4\% & MVC: & MVC: & MVC: & MVC: & MVC: \\
& 19.2 & 23.1 & 24.2 & 26.6 & 29.1 \\
& $(13.9-34.9)$ & $(11.9-37.9)$ & $(12.2-43.8)$ & $(16.3-36-$ & $(15.5-43.5)$ \\
& TDF/FTC: & TDF/FTC: & TDF/FTC: & $2)$ & TDF/FTC: \\
& 18.8 & 23.1 & 24.5 & TDF/FTC: & 23.3 \\
& $(9.8-32.2)$ & $(10.9-39.4)$ & $(10.4-41.1)$ & 24.7 & $(15.9-43.1)$ \\
CD4 gain & MVC: & MVC: & MVC: & MVC: & MVC: \\
(cells/ $\mu \mathrm{L})$ & 0 & 167 & 192 & 247 & 256 \\
& TDF/FTC: & TDF/FTC: & TDF/FTC: & TDF/FTC: & TDF/FTC: \\
& 0 & 110 & 175 & 203 & 189 \\
HIVRNA $<$ & MVC: & MVC: & MVC: & MVC: & MVC: \\
50 copies/ & 0 & $21 \%$ & $82 \%$ & $100 \%$ & $100 \%$ \\
ml & TDF/FTC: & TDF/FTC: & TDF/FTC: & TDF/FTC: & TDF/FTC: \\
& 0 & $11 \%$ & $53 \%$ & $100 \%$ & $100 \%{ }^{\mathrm{a}}$ \\
\hline
\end{tabular}

a $93 \%$ in ITT analysis, considering discontinuation as failure

Conclusions: NRTIs-sparing regimen with Maraviroc $150 \mathrm{mg}$ QD and lopinavir/ritonavir is similar in efficacy and tolerability to conventional treatment in naïve-patients. A more favourable trend in immunological recovery was observed but it needs to be confirmed in larger samples. Regimens NRTIs-sparing in HIV-infected patients naïve to antiretroviral therapy should be explorated.

\section{SC 08 \\ MODULATORY EFFECTS OF THE NOVEL CCR5 ANTAGONIST MARAVIROC ON MICROGLIAL PRO-INFLAMMATORY ACTIVATION ELICITED BY GP120 \\ L. Lisi ${ }^{\prime}$, P. Navarra ${ }^{l}$, C. Dello Russo ${ }^{l}$}

${ }^{1}$ Instituto di Farmacologia, Università Cattolica del Sacro Cuore, Rome, Italy

Despite the success of the highly active antiretroviral therapy (HAART) in reducing peripheral viral load as well as improving CD4+ counts, neurological disorders, involving both the central and peripheral nervous system, still affect approximately 50\% of HIVinfected patients. Neuroinflammation, a process mainly initiated and sustained by infected-activated microglial cells, plays a prominent role in the physiopathology of HIV-1 related neurological disorders. Microglial cells, the macrophages resident in the central nervous system, also represent a virus reservoir. Infected microglial cells become activated and release a vast array of pro-inflammatory mediators as well as HIV-1 proteins, that sustain cell activation and mediate neuronal damage. Among the latter, the glycoprotein gp120 is believed to play a crucial role. Gp120 is the envelope protein that allows the virus entry in the host cells via binding to the CD4 receptor together with a specific co-receptor (CCR5/CXCR4). Different strains of HIV-1 viruses have been characterized for their differential ability to infect host cells. Briefly, the beta-chemokine receptor, CCR5, is the major co-receptor for the macrophage (M)-tropic (R5) strains of HIV1 , whereas the alpha-chemokine receptor, CXCR4, facilitates entry of T-tropic (X4) HIV-1 strains. Small molecular weight inhibitors of the CCR5 co-receptor represent a new class of antiretroviral drugs, among which Maraviroc is in clinical use. By preventing CCR5 binding, these drugs abort HIV-1-cell membrane fusion and interrupt the HIV-1 replication cycle. By interfering with a chemokine receptor that is highly expressed on microglial cells, these drugs have therefore the potential to reduce microglial pro-inflammatory activation during HIV-1 infection. To test this hypothesis, we used primary cultures of rat cortical microglial cells activated by different isoforms of gp120 derived from $\mathrm{M}$ and T-tropic viruses. Gp120CN54, a M-tropic derived gp120, showed the highest pro-inflammatory effects in this paradigm, thus it was used to test the antiinflammatory potential of maraviroc. The latter displays opposite effects, depending on whether or not interferon-gamma (IFNgamma) was also present in the system; indeed, cytokines like IFNgamma, mainly produced by active CD4+Th1 T cells, contribute to exacerbate microglial proinflammatory activation during HIV disease clinical course. While maraviroc consistently reduces pro-inflammatory effects of Gp120CN54, it increases the pro-inflammatory activation elicited by the co-administration of Gp120CN54 and IFNgamma. Taken together, these data suggest a potential beneficial use of maraviroc in the early phase of HIV infection, when cytokine production (including IFNgamma) in the brain is lower, thus providing additional benefits in preventing neuropathology associated with HIV infection.

\section{SC 09}

MONITORING OF VIROLOGICAL AND PHARMACOLOGICAL CEREBROSPINAL FLUID PARAMETERS IN HAART-TREATED HIV-POSITIVE PATIENTS IN THE CLINICAL SETTING

A. Calcagno ${ }^{\prime}$, S. Bonora ${ }^{l}$, M.C. Tettoni ${ }^{1}$, M. Bonasso ${ }^{\prime}$, B. Salassa ${ }^{\prime}$,

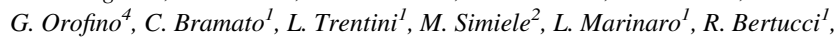
D. Imperiale ${ }^{3}$, A. D'Avolio ${ }^{2}$, V. Ghisetti ${ }^{5}$, G. Di Perri ${ }^{1}$

${ }^{1}$ Department of Infectious Diseases, University of Torino, Turin, Italy;

${ }^{2}$ Pharmacokinetics and Pharmacogenetics Laboratory, University of Torino, Turin, Italy; ${ }^{3}$ Department of Neurology, Ospedale Maria Vittoria, ASLTO2, Turin, Italy; ${ }^{4}$ Department of Infectious Diseases, "Divisione A", ASLTO2,

Turin, Italy; ${ }^{5}$ Molecular Biology and Virology Laboratory, Ospedale Amedeo di Savoia, ASLTO2, Turin, Italy

Background: HIV-associated neurocognitive disorders have been showed to be highly prevalent even in HAART treated patients. Since several factors related to subjects, viruses and drugs characteristics have been associated to these phenomena, monitoring of cerebrospinal fluid (CSF) parameters should be considered in neurologically impaired patients.

Methods: Samples from HIV-positive patients undergoing lumbar punctures for clinical reasons were analyzed for plasma and CSF viral 
loads (with a limit of detection of 20 copies/ml), genotype resistance testing and predicted tropism, BBB integrity indexes (Albumin, IgG and Tourtelotte) and for plasma and CSF concentrations of antiretrovirals (measured through a validated HPLC-MS method). CNS Penetration-Effectiveness Score (CPE) was calculated according to the 2010 version. Data are expressed as median (interquartile range); Spearman's analysis were used to test association between variables. Results: Fifty patients on different HAART regimens undergoing lumbar punctures for diagnostic reasons were included in this analysis. Median age and CD4 were 44 years (37-51) and 216 (66-524) cells $/ \mu \mathrm{l} ; 60 \%$ of them were male. Samples were collected 2-24 h after drug intake. CSF and plasma viral load (VL) were respectively $2.14(1.27-3.32)$ and $1.8(1.27-3.19)$ Log copies/ml; the VL CSF-to-plasma ratio ranged from 0.28 to 7.20. Among virologically suppressed patients (24/50), 20.8\% showed a detectable CSF VL (ranging from 74 to 950 copies $/ \mathrm{ml}$ ). CPE score in this cohort was 7 (6-8) while $80 \%$ of patients with CSF escape had a CPE score $=7$. Blood-brain barrier (available in 35 pts) was impaired in 12 (34.3\%) with albumin and $\mathrm{IgG}$ ratios respectively of $0.52(0.37-0.84)$ and 0.54 $(0.36-0.77)$; inflammatory patterns were seen in $57.1 \%$ of them. CSFto-plasma ratios of antiretrovirals were substantially comparable to the ones previously reported except for higher raltegravir ratios $[0.21$ $(0.10-0.29)]$. Cerebrospinal drug levels were below $\mathrm{IC}_{50}$ more frequently in PI intakers (atazanavir 7/10, lopinavir 7/11 and darunavir 4/9) as compared to newer drugs treated ones (raltegravir 1/17, maraviroc $0 / 4$, etravirine $0 / 1$ ).

Conclusion: Our results in a very heterogeneous cohort of HAARTtreated patients show a great variability in CSF parameters. The cerebrospinal evaluation of virologically suppressed patients revealed that $20.8 \%$ of them had a detectable viral load and a slightly lower median CPE (6 vs. 7) as compared to concordant virus controllers. A relevant proportion of boosted PIs-treated patients (60\%) showed CSF PI concentration below the $\mathrm{IC}_{50}$; conversely a higher than previously reported passage of raltegravir was noted (21 vs. 3-5\%). This high variability should be related to individual characteristics, co-administered drugs and blood-brain barrier impairment (that was present in a significant proportion of subjects). The clinical impact of these observations should be evaluated in long term follow-up prospective studies.

\section{SC 10 \\ EFFICACY, SAFETY, DISCONTINUATION RATES AND PHARMACOKINETICS OF BOOSTED OR UNBOOSTED ATAZANAVIR WHEN COMBINED WITH A TENOFOVIR OR ABACAVIR CONTAINING BACKBONE

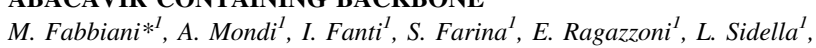 M. Doino ${ }^{I}$, P. Navarra ${ }^{I}$, R. Cauda ${ }^{I}$, S. Di Giambenedetto ${ }^{I}$ \\ ${ }^{1}$ Università Cattolica del Sacro Cuore, Rome, Italy}

Introduction: Atazanavir (ATV) is frequently prescribed boosted or unboosted in association with a tenofovir (TDF) or abacavir (ABC) containing backbone. We investigated efficacy, safety, discontinuation rates and pharmacokinetics of these regimens in routine clinical practice.

Methods: We retrospectively selected patients treated with ATV plus an NRTI backbone. Four regimens were compared: (1) ATV/r+TDFbased backbone; (2) ATV/r+ABC-based backbone; (3) ATV+TDFbased backbone; (4) ATV+ABC-based backbone. Patients were followed from ATV starting to last available visit or regimen discontinuation. Clinical and laboratory variables, reasons for discontinuation and pharmacokinetic parameters were compared between groups.

Results: A total of 748 patients $(55.3 \%$ males, median age 44 years IQR 38-48, 33.3\% with past AIDS-defining events, median HIV-
RNA 49 copies/mL IQR 49-13,844, median CD4 405 cells/ $\mu$ L IQR 260-594 at ATV starting) were analyzed, of which $529(70.7 \%)$ in group 1, $44(5.9 \%)$ in group 2, $47(6.3 \%)$ in group 3 and $128(17.1 \%)$ in group 4. At baseline no significant between groups differences were observed regarding HIV-RNA and CD4. Median observation time was 15.4 months (IQR 4.9-31.5). Overall, 55.2\% of patients discontinued ATV during follow up, of which $29.8 \%$ in the first year of therapy. Virologic failure and toxicity were responsible for treatment discontinuation in 2.1 and $16.4 \%$ of patients, respectively. At the end of follow up HIV-RNA and CD4 cells count did not significantly differ between groups. Survival curves showed a significant between groups difference in the probability of regimen discontinuation $(\mathrm{p}=0.002)$, with higher risk for group $2(46 \%$ at 24 months) and lower for group 4 (14\% at 24 months). At multivariate analysis, subjects with past AIDS-defining events (HR 1.33, p =0.007) and group 2 patients (HR 1.87 when compared to group $1, \mathrm{p}=0.001$ ) showed an higher risk of discontinuation, while those with baseline HIV-RNA $<50$ copies/mL showed a lower risk (HR 0.89, $\mathrm{p}=0.040$ ). Subtherapeutic ATV levels were associated with higher risk of treatment failure only at univariate analysis. Group 2 patients showed an independent higher risk of treatment failure or toxicity when compared to group 1 (HR 20.5, p $=0.015$ and HR 2.01, p =0.018, respectively). No significant differences in lipid parameters were observed between boosted and unboosted regimens; patients on TDF showed lower total cholesterol $(\mathrm{p}=0.009)$ and HDL levels $(\mathrm{p}=0.012)$, and a higher decrease in GFR as estimated by MDRD formula (mean change -8 vs. $-3 \mathrm{~mL} / \mathrm{min} / 1.73 \mathrm{~m}^{2}$ ). Patients on boosted regimens showed less frequently subtherapeutic ATV levels $(p<0.001)$ but more frequently potentially toxic levels $(p=0.005)$. Conclusions: ATV-based regimens showed good efficacy and tolerability. Patients on $\mathrm{ABC}$ showed higher rates of treatment discontinuation for any reason, virological failure and toxicity when compared to TDF. TDF treated patients showed lower lipid levels but higher decrease in GFR.

\section{SC 11}

\section{NEUROIMAGING OF HIV/AIDS PATIENTS TODAY}

R. Barbo ${ }^{*}{ }^{1}$, C. Agostinis ${ }^{2}$, B. Marini ${ }^{3}$, M.R. Rottoli ${ }^{2}$

${ }^{I}$ Neuroradiologia, Humanitas Gavazzeni, Bergamo, Italy; ${ }^{2}$ Neuroradiologia, Ospedali Riuniti, Bergamo, Italy; ${ }^{3}$ Malattie Infettive, Ospedali Riuniti,

Bergamo, Italy

Since the early 1980s Neuroimaging has achieved high specificity in the diagnosis of cerebral HIV/AIDS-related lesions, thanks to improvement of morphologic techniques, functional MR sequences and metabolic studies provided by nuclear medicine. This allows the clinician a more accurate diagnosis, thus reducing the necessity for cerebral biopsy. On the other hand, the specificity of some HIV/ AIDS-related imaging findings can allow the neuroradiologist to correctly address the patient to the infectivologist. Antiretroviral drugs (HAART) have improved the chances of survival and the quality of life of AIDS patients and have dramatically reduced the incidence of complications. The immunoreconstruction induced by new drugs has modified some histopathologic features and, as a consequence, the neuroradiologic aspects of classic features. Moreover, a new syndrome, called IRIS (immune reconstitution inflammatory syndrome) due to restoration of immunity to specific infection and non-infection antigens has been described in a small percentage of patients with AIDS. This condition is of profound clinical significance and must be diagnosed promptly. Neuroimaging plays a crucial role in the diagnosis and follow up of these patients, by monitoring treatment response, identifying disease progression and predicting prognosis, in this new syndrome as well as in the other classic complications occurring in HIV/AIDS setting. 


\section{SC 12}

ALEXITHYMIA AS A MAJOR CARDIOVASCULAR RISK FACTOR IN HIV-INFECTED PATIENTS

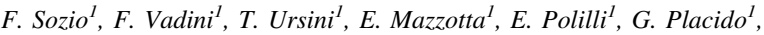
A. Agostinone ${ }^{1}$, L. Manzoli ${ }^{2}$, G. Parruti $*^{l}$

${ }^{1}$ U.O. Malattie Infettive, Pescara, Italy; ${ }^{2}$ Dipartimento di Epidemiologia e Sanità Pubblica, Università di Chieti, Chieti, Italy

Many factors are known to contribute to faster progression of cardiovascular (CV) aging and disease in HIV infected patients. In addition to traditional $\mathrm{CV}$ risk factors, we investigated for the first time in a single-site Italian HIV cohort several psychological factors including Alexithymia, TypeD personality, mental and physical components (MCS and PCS) of quality of life (QoL) and depression, in parallel with intima-media thickness of carotid arteries. HIV infected patients were consecutively enrolled from February to December, 2010. Single operators assessed both carotid intima-media Thickness and the presence of plaque(s) by B-mode ultrasonography, as well as the psychological factors, including Alexithymia, using the 20-item Toronto-Alexithymia-Scale (TAS-20, cut-off $=50$ ), TypeD personality, using the DS14 Distress Scale (Negative Affectivity Scale, cut-off $=9$; Social Inhibition Scale, cut-off $=9$ ), depression symptoms, using the Beck depression inventory (BDI, cut-off $=15$ ) and QoL, using the SF12 questionnaire. All statistical analyses were carried out using the Stata 10.0 package. We enrolled 213 patients, $73.7 \%$ males, aged $44.9 \pm 10.1$ years with a BMI of $24.1 \pm 3.9$. Mean duration of HIV-infection was $13.8 \pm 6.9$ years, in $71.4 \%$ of cases due to sexual exposure; $30.0 \%$ of patients had an AIDS-defining event and a Nadir CD4 of $254.3 \pm 13.6$ cells $/ \mathrm{mmc}$. HCV infection was diagnosed in $26.8 \%$ of them; smokers were $56.4 \%$, diabetics $21.4 \%$, hypertensive $25.1 \%$, hypercholesterolemic $29.7 \%$. As to HAART, $54.0 \%$ had detectable HIV RNA, $15.1 \%$ being untreated. Carotid plaques $(\mathrm{CP})$ were found in $39.3 \%$ of patients, bilaterally in $14.2 \%$; TypeD personality was diagnosed in $35.6 \%$, Alexithymia in $41.2 \%$, depression in $31.9 \%$ of patients. Patients with $\mathrm{CP}$ were significantly older $(52.2 \pm 9.5$ vs. $41.5 \pm 8.0, \mathrm{p}<.001)$, hypertensive (30.5 vs. $65.2 \%, \mathrm{p}<.001)$, comorbid for at least one condition (20 vs. $48 \%, \mathrm{p}<.001)$, diabetics $(\mathrm{p}=.025)$, lipodystrophic $(\mathrm{p}=.02)$, with AIDS diagnosis $(\mathrm{p}=.06)$; glycemia, total cholesterol and triglycerides were also significantly associated. Patients with Alexithymia (TAS score $>50$ ) had significantly more CPs (26.9 vs. $60.9 \%$, $\mathrm{p}<.001)$, whereas other psychological factors were not associated with CPs. Stepwise multivariate logistic regressions confirmed that age (OR 1.15, 95\% CI 1.07-1.22, p <.001), Alexithymia (OR 4.6, 95\% CI 1.86-11.33, $\mathrm{p}<.001$ ), total cholesterol (OR 1.01, CI $1.0-1.02, \mathrm{p}=.003)$, male sex (OR 3.29, 95\% CI 1.14-9.50, $\mathrm{p}=.03$ ), hypertension (OR 3.14, CI 1.11-8.9, $\mathrm{p}=.03$ ) duration of HIV infection (OR $1.07,95 \%$ CI $1.00-1.14, \mathrm{p}=.04)$, confirmed their independent association. On a monocentric Caucasian population, Alexithymia was tightly associated with the presence of CPs, more remarkably than most traditional $\mathrm{CV}$ risk factors. Its diagnosis appears to usher a new tool for a better management of CV risk and disease in HIV patients.

\section{SC 13}

MONITORING OF DRUG RESISTANCE MUTATIONS IN PROVIRAL DNA OF PATIENTS RESPONDING TO ANTIRETROVIRAL THERAPY

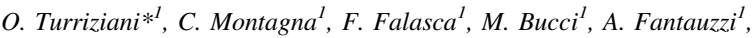

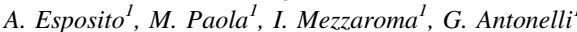

${ }^{I}$ Sapienza University of Rome, Rome, Italy

Several studies in patients on successful antiretroviral therapy (ART) but with a history of drug-resistance, provided evidence for the dynamic nature of the latent reservoir and showed that any viral variant, including drug-resistant variants, will enter the reservoir and remain conserved. Some studies have showed that, when the viral load is below the detection limit, antiretroviral resistance does not occur, whereas other show that resistant related mutations can indeed emerge under such conditions. In order to gain new insights into the dynamic of archived resistant viral variants occurring during suppressive ART, we evaluated the evolution of drug resistance mutations in proviral DNA in 20 patients treated with therapeutic regimen which included Darunavir (DRV) + Raltegravir or DRV combined with NRTI or with NNRTI. All subjects had already been treated with several antiretroviral drugs, with a mean number of $6.4 \pm 2.0$ NNRTI and $4.8 \pm 2.2 \mathrm{PI}$, and had been receiving antiretroviral treatment for a long time (mean period 17 years; range 12-24 years). Eleven patients were also experienced for NNRTI. During follow-up all subjects had plasma viremia $<50$ copies $/ \mathrm{ml}$. In only two patients an intermittent low viremia was observed during follow up. Proviral DNA was extracted and amplified from peripheral blood mononuclear cells (PBMC) and sequenced by the TruGene assay (Siemens Heathcare Diagnostics). The analysis was performed at baseline, and after 6 and 18 months of therapy. At baseline all patients showed drug resistance mutations in the proviral DNA. All mutations were related to ART previously received. Specifically, considering the whole population, at time 0 the number of mutations detected in proviral DNA was $12.2 \pm 5.17$ and after 18 months of therapy the total number of mutations was $12.6 \pm 5.0$. Interestingly in 10 of $20(50 \%)$ the number of mutations increased and decreased in 7 of $20(35 \%)$. Specifically, in 11 patients the change in resistance mutations number was observed both in RT and PRO region; in 4 patients only RT mutations number changed as well as in 2 individuals only PRO mutations number differed from the number detected at baseline. In only 3 individuals the number of mutations at 18 months was the same to the number detected at baseline, but the type of mutations were different. Although performed on a small patient population, these data suggest that the mutational archive in HIV-DNA from PBMC can change during suppressive ART. The long therapeutic history of these patients and the high number of resistant viral variants archived in PBMC does not allow to claim that the appearance of new mutations, observed in some patients, was due to the selection of new viral variants. However the appearance and disappearance of drug resistance mutations, suggest that: HIV replication continue at low levels despite therapy, and/or long-lived cells containing HIV proviruses produce HIV at low levels after reactivation.

\section{SC 14}

DYNAMICS AND COMPARTMENTALIZATION OF CXCR4-USING HIV VARIANTS DURING THE EARLY PHASES OF HIV INFECTION, ESTABLISHED BY ULTRA-SENSITIVE NEXT GENERATION SEQUENCING

G. Rozera ${ }^{\prime}$, I. Abbate ${ }^{1}$, C. Vlassi $^{2}$, A. Bruselles ${ }^{1}$, B. Bartolini ${ }^{1}$, E. Giombini ${ }^{l}$, A. Corpolongo ${ }^{2}$, G. D'Offizi ${ }^{2}$, P. Narciso ${ }^{2}$, M.R. Capobianchi ${ }^{I}$

${ }^{1}$ Laboratory of Virology of INMI L.Spallanzani, Rome, Italy; ${ }^{2} I V$ Division,

Clinical Department of INMI L.Spallanzani, Rome, Italy

Aims: To establish HIV-1 RNA and proviral DNA dynamics in primary infection and to assess quasispecies composition (V3 diversity and proportion of $\mathrm{X} 4$ variants) in circulating and GALT-associated HIV.

Methods: Twenty acutely infected patients were enrolled within 4 weeks from seroconversion (T0). Ten patients remained free of therapy, 10 started HAART based on clinical presentation. Viral quasispecies in plasma RNA and in PBMC proviral DNA was assessed at T0 and at 6 months (T6) by V3 ultra-deep pyrosequencing. Viral quasispecies was also assessed at T0 in GALT proviral DNA. Prediction of co-receptor usage was performed by PSSM.

Results: At T0, the amount of HIV-1 RNA and proviral DNA in PBMC were positively correlated $(\mathrm{r}=0.457, \mathrm{p}=0.043)$; no 
correlation between CD4 and HIV-1 RNA was observed. Half of patients displayed $\mathrm{X} 4$ variants above $0.3 \%$ in viral RNA quasispecies, with intra-patient median proportion of $\mathrm{X} 4$ variants of $0.31 \%$ (range $0.30-56.25 \%$ ); $7 / 12$ patients displayed $\mathrm{X} 4$ variants $>0.3 \%$ in PBMC proviral DNA, median proportion $0.99 \%$ (range $0.30-3.55 \%$ ); $2 / 5$ patients displayed $\mathrm{X} 4$ variants $>0.3 \%$ in GALT proviral DNA, median proportion $0.30 \%$ (range $0.30-0.87 \%$ ). Compartmentalization of viral quasispecies could be observed in patients with high diversity. In patients who underwent early treatment, baseline CD4 cells were lower as compared to those who remained untreated $(\mathrm{p}=0.028)$. Consistently, the T0 V3 diversity was significantly higher in both circulating viral and proviral sequences $(627.5$ vs. 71.5 mean substitutions/site $\times 10^{-4}, \mathrm{p}=0.019$ and 423 vs. 29 mean substitutions/ site $\times 10^{-4}, \mathrm{p}=0.018$, respectively). At T6, in patients undergoing HAART the mean decline of HIV-1 RNA and DNA was 3.69 Log copies/ml and 0.6 Log copies/106 PBMC, respectively, while in the untreated patients the RNA viral load virtually remained unchanged ( $0.07 \log$ copies $/ \mathrm{ml}, \mathrm{p}<0.001$ vs. the treated group), while proviral DNA showed a modest increase $(0.31 \mathrm{Log}$ copies $/ 106$ PBMC, $\mathrm{p}=0.040$ vs. the treated group). Consistently, in patients who remained free of therapy, the diversity of PBMC proviral DNA, but not that of viral RNA sequences, significantly increased from T0 to T6. Although overall $(\mathrm{T} 0+\mathrm{T} 6)$ diversity and proportion of $\mathrm{X} 4$ variants were positively correlated $(r=0.382, p=0.004)$, the numbers were insufficient, at present, to compare the trend of X4 frequency in the two groups during the observation period.

Discussion: Our findings show that $\mathrm{X} 4$ variants may be commonly present, with very broad range of intra-patient frequency, during the early phases of HIV infection. Compartmentalized distribution between GALT and blood cells, as well as filling of proviral DNA in cellular reservoirs and diversification of viral quasispecies are early events during acute infection.

\section{SC 15}

THE GENOTYPIC FALSE POSITIVE RATE DETERMINED BY POPULATION V3-SEQUENCING CAN PREDICT THE BURDEN OF X4 QUASISPECIES DETECTED BY PYROSEQUENCING

V. Cento ${ }^{* 1}$, V. Svicher ${ }^{1}$, G. Rozera ${ }^{2}$, I. Abbate ${ }^{2}$, M.M. Santoro ${ }^{1}$, D. Armenia ${ }^{\text {, }}$, L. Fabeni ${ }^{2}$, G. Palamara ${ }^{3}$, A. Latini ${ }^{3}$, G. Rizzardini ${ }^{4}$, V. Micheli ${ }^{4}$

A.R. Buonomini ${ }^{5}$, M. Andreoni ${ }^{5}$, C.F. Perno ${ }^{\text {I,2,5 }}$, M.R. Capobianchi, ${ }^{2}$,

F. Ceccherini-Silberstein ${ }^{1}$

${ }^{I}$ Department of Experimental Medicine and Biochemical Science, University of "Tor Vergata", Rome, Italy; ${ }^{2}$ I.N.M.I. "L. Spallazani", Rome, Italty; " "San Gallicano" Hospital, Rome, Italy; ${ }^{4}$ "L. Sacco" Hospital, Milan, Italy;

${ }^{5}$ University Hospital "Tor Vergata", Rome, Italy

Background: The range 10-100 of false-positive rate (FPR) is a so far qualitative percentage score provided by Geno2Pheno-algorithm that positively predicts the use of the CCR5-coreceptor. We evaluated the potential correlation between FPR obtained by population V3genotyping and the presence of HIV-1 variants with DM/X4-tropism, detected by both enhanced-sensitivity trofile assay (ESTA) and ultradeep V3-pyrosequencing (UDPS).

Methods: This study included 46 HIV-1 infected (all B-subtype) patients, naïve to maraviroc, with viremia $>10,000 \mathrm{copies} / \mathrm{ml}$. For each patient, HIV tropism was assessed by ESTA, standard population V3-sequencing and UDPS with GS-FLX Roche. For UDPS, only V3 coding sequences detected above $0.3 \%$ of viral-species were analyzed. We particularly focused on $\mathrm{X} 4$ variants with prevalence $>10 \%$ known to be clinically relevant for response to maraviroc (Swenson 2009). The association between FPR and X4 variants was assessed by Chi-square tests. Geno2pheno set at cut-off 5 was used to predict viral tropism.

Results: Tropism prediction by UDPS was 93.0 and $79.1 \%$ concordant with population V3-genotyping and ESTA, respectively. By UDPS, R5 variants were always detected in all samples, irrespective of FPR values. In particular, R5 species were detected in all $8 / 8$ patients with FPR $<5$, even in some cases as predominant species (prevalence ranging from 6.1 to $87.9 \%$ ), and were present with a prevalence $>80 \%$ in all the patients with FPR $>20$. By contrast, X4 variants progressively decreased by increasing the FPR $(\mathrm{p}<0.001)$. In particular, X4 variants were undetectable by both UDPS and ESTA in all 12 patients with FPR $>60$, and were present at levels $>10 \%$ in 2/16 (12.5\%) patients with FPR 20-60, in 2/6 (33.3\%) with FPR $10-20$, in $2 / 4(50.0 \%)$ with FPR $5-10$, in $3 / 3(100 \%)$ with FPR $2-5$ and in $5 / 5(100 \%)$ patients with FPR $<2$. In this latter group of patients, X4 species showed prevalence ranging from 37.3 to $93.9 \%$ of the entire viral population.

Conclusions: FPR determined by population V3-sequencing can predict the burden of $\mathrm{X} 4$ variants. No $\mathrm{X} 4$ variants are detected in patients with FPR $>60$, thus suggesting that patients with higher FPR have even greater chances of increased activity of CCR5 inhibitors. In addition, the presence of $\mathrm{R} 5$ viruses in all patients, including those with very low FPR, confirms the greater pathogenetic relevance of such strains compared to X4. Overall, these results suggest that genotypic tropism testing may provide further information (beyond the classical R5 versus $\mathrm{X} 4$ tropism definition) for an optimal positioning of CCR5-antagonists in clinical practice.

\section{SC 17}

\section{IMPACT OF MUTATIONS OUTSIDE V3 ON CORECEPTOR} TROPISM PHENOTYPICALLY ASSESSED IN SUBTYPE B HIV-1INFECTED PATIENTS

L. Monno ${ }^{1}$, A. Saracino $*^{2}$, L. Scudeller ${ }^{3}$, G. Punzi $^{1}$, A. Lagioia ${ }^{1}$, G. Brindicci $^{1}$, G. Angarano ${ }^{1}$

${ }^{1}$ Clinica Malattie Infettive, Università degli Studi di Bari, Bari, Italy; ${ }^{2}$ Clinica Malattie Infettive, Università degli Studi di Foggia, Foggia, Italy; ${ }^{3}$ IRCCS

Policlinico San Matteo, Pavia, Italy

Coreceptor tropism (CTR) is increasingly deduced from the genotype on the basis of V3 alone. However, mutations outside V3 can conceivably influence CTR, possibly explaining discordant results between genotypic and phenotypic assays. This study analyzes the impact of mutations outside V3 on CTR as determined by the enhanced sensitivity trofile assay (ESTA). Paired ESTA (Monogram BioSciences) and gp120 sequences (population sequencing; codon 32 of $\mathrm{C} 1$ to V5) were obtained from 47 newly diagnosed subtype B HIV1-infected patients; all were naïve to antiretroviral therapy and included $30 \%$ with AIDS. For gp120 sequence analysis, nucleotide mixtures were considered when the second highest electropherogram peak was $>25 \%$; sequences were translated into all possible permutations giving a total of 342 sequences. Relationship between ESTA and patient- and sequence-related variables was assessed by logistic regression (clustering for patient was considered). Sequence positions were tested with simple correspondence analysis and those with the highest inertia (variance; namely, upper decile) were retained in multiple correspondence analysis (MCA). Only mutations with mass (frequency) $>0.01$ are reported. MCA is an exploratory analysis for categorical data to detect/represent associations between variables in a data set as points in a low-dimensional (i.e., 2 or 3 dimensions) Euclidean space. Associations between variable categories are represented graphically as "maps", where the first axis is the most important dimension, the second axis the second, etc. Proximity between map points indicates that these variable categories tend to appear together in the data set. ESTA identified R5 and dual/mixed (DM) viruses in 66 and $34 \%$ of patients, respectively; no pure X4 strain was labeled. $43 \%$ of AIDS patients had a R5 strain. At univariate analysis, of patient variables, only $\mathrm{CD} 4+$ was associated with ESTA $(p=0.014)$; of sequence variables, V3 net charge was associated $(\mathrm{p}=0.001)$ with CTR along with substitutions at positions 132, 140, 145 (V1, HXB2 numbering), 164, 169 (V2), 399, 400, 403, 404, 406, 407, 410 (V4), 461 and 464 (V5) which were kept for 
subsequent analysis. MCA suggested that, although the level of variance explained by the first two dimensions was low (27\%), with the first three dimensions accounting for only $40 \%$ of inertia, mutations apparently associated to DM were $140 \mathrm{~N}, 145 \mathrm{~S}, 169 \mathrm{M} / \mathrm{V}$, 399G, 400T, 406T, 407D and 461N. To our knowledge, this is the first report investigating all gp120 regions; sequences with all possible permutations from each patient were included. Although MCA is exploratory, and the sample size was small, some mutations can be associated with ESTA. However, the variance level explained by the included variables was relatively low. Thus, extending the analysis to include pure $\mathrm{X} 4$ and expanding the sample size would be desirable to allow formal statistical testing of the multivariate associations.

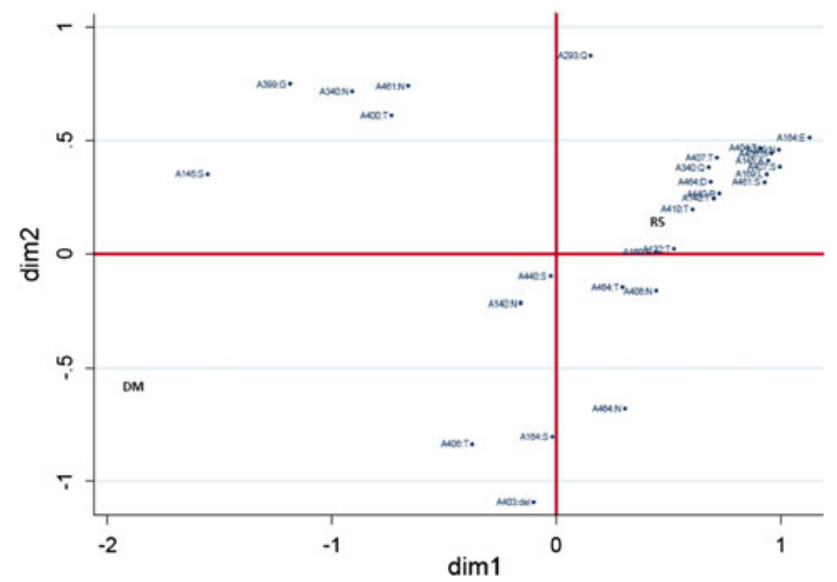

\section{SC 18}

SIGNATURE MUTATIONS IN V3 AND BRIDGING SHEET DOMAIN OF HIV-1 GP120 HIV-1 ARE SPECIFICALLY ASSOCIATED WITH DUAL TROPISM AND MODULATE THE INTERACTION WITH CCR5 N-TERMINUS

C. Alteri $*^{l}$, A. Artese ${ }^{2}$, J.M. Zhang ${ }^{3}$, F. Mercurio ${ }^{1}$, G. Costa ${ }^{2}$, F. Stazi ${ }^{1}$, L. Fabeni ${ }^{4}$, A. Bertoli ${ }^{I}$, F. Forbici ${ }^{4}$, R. Salpini ${ }^{I}$, S. Dimonte ${ }^{2}$, S. Alcaro ${ }^{2}$, F. Ceccherini-Silberstein ${ }^{l}$, C.F. Perno ${ }^{l}$, V. Svicher ${ }^{1}$

${ }^{\prime}$ Department of Experimental Medicine, University of Rome 'Tor Vergata',

Rome, Italy; ${ }^{2}$ Department of Pharmacobiological Sciences, University of Catanzaro "Magna Graecia", Roccelletta di Borgia, CZ, Italy; ${ }^{3}$ Department of Statistics, Yale University, New Haven, USA; ${ }^{4}$ INMI L Spallanzani, Infectious Diseases Division, Rome, Italy

Background: HIV-1 dual-tropic species predominate in a significant proportion of patients, and have peculiar properties in term of response to entry inhibitors. As we recently characterized V3 genetic determinants correlated with pure-R5 and -X4 tropism, we now define gp120 genetic determinants and structural features underlying the characteristics of dual-tropism in vivo.

Methods: This study includes 498 V3 and 242 gp120 sequences from 740 HIV-1 B subtype infected patients, retrieved from Los Alamos HIV Database, all with phenotypic determination of HIV tropism. Geno2pheno (set at a false positive rate of $10 \%$ ) was used to confirm viral tropism. Gp120-interaction with CCR5 N-terminus was evaluated by docking-analysis starting from the model described in Huang, Science 2007.

Results: We identified new V3 determinants significantly correlated with phenotypically-defined dual-tropism. This is the case of T2M, I26R, and I12V occurring in 0,0 , and $2 \%$ respectively of both pure R5- and X4-viruses, and in 9.3, 4.9, and 14.2\%, of dual-tropic viruses, respectively ( $\mathrm{P}<0.001$ after correction for multiple comparison).
Among them, both I12V and I26R decrease CCR5 N-terminus binding affinity for gp120 (crucial for HIV entry via CCR5-coreceptor) with respect to pure-R5 virus (I12V: $-6.60 \mathrm{kcal} / \mathrm{mol}$; I26R: $-6.10 \mathrm{kcal} / \mathrm{mol}$; WT: $-6.90 \mathrm{kcal} / \mathrm{mol}$ ). I12V also increases the distance between Arginine at V3 position 30 and Sulphotyrosine at CCR5-position 10 (crucial for proper binding between gp120 and CCR5), thus affecting the interaction between these two important residues. Outside the V3 region, mutations T102S, M105V, and R398Q, all in gp120 bridging sheet domain, specifically correlate with dual-tropism. These mutations occur in $<3 \%$ of both pure R5and X4-viruses, and in 15.8, 16.7, and $33.3 \%$ of dual-tropic viruses, respectively. In addition, they form a tight cluster of mutations observed only in dual-tropic viruses (bootstrap $=1.0$ ). Among them, R398Q strongly affects CCR5 N-terminus binding affinity to gp120 with respect to pure-R5 virus (R398Q: $-5.70 \mathrm{kcal} / \mathrm{mol}$; WT: $-6.90 \mathrm{kcal} / \mathrm{mol})$.

Conclusions: Specific determinants in $\mathrm{V} 3$ and bridging sheet modulate HIV-1 dual-tropism in vivo and gp120 interaction with CCR5 $\mathrm{N}$-terminus, suggesting that dual-tropic viruses often represent HIV species structurally different from pure R5- or X4-viruses (rather than just their mixture). This information is useful for a finer optimization of genotypic-tropism testing, and may have molecular-implication for designing new entry-inhibitors.

\section{SC 19}

DEVELOPMENT OF RECOMBINANT VIRUS ASSAYS FOR PHENOTYPIC EVALUATION OF THE SUSCEPTIBILITY TO INTEGRASE INHIBITORS AND HIV CORECEPTOR TROPISM F. Saladini $*^{1}$, A. Rosi ${ }^{1}$, I. Vincenti ${ }^{1}$, G. Meini ${ }^{I}$, M. Zazzi ${ }^{1}$

${ }^{I}$ Section of Microbiology, Department of Biotechnology, University of Siena, Siena, Italy

Background: Despite the dramatic success of HAART, several factors can still compromise the efficacy of therapy ultimately leading to development of drug resistance. This problem has fostered the development of new classes of drugs like integrase inhibitors (INI) and co-receptor antagonists (CA). Notwithstanding its impressive clinical success, Raltegravir (RAL), the first FDA-licensed INI, can easily select for drug-resistant virus variants. Although mutations responsible for drug resistance have been characterized the correlation between integrase mutation profiles and INI resistance is still partial, warranting further investigation through phenotypic testing. A similar concern applies to Maraviroc (MRV), the first FDA-approved CA, which prevents virus binding to the cell membrane coreceptor CCR5. Since MRV is only active if the patient's virus is classified as CCR5-tropic, HIV tropism must be preliminary assayed, either inferred through genotypic tests or determined by phenotypic assays. Genotype-based algorithms are gaining acceptance but still lack sensitivity for minority species and may have limitations in the accuracy of tropism prediction. Thus, phenotypic assays maintain their role in direct tropism determination and improvement of genotypic assays through genotype-phenotype correlation studies.

Methods: We have developed recombinant virus assays (RVA) both for the estimation of drug resistance to INI and for the characterization of viral tropism. For these purposes, pNL4-3-derived vectors harboring deletion in the integrase gene and in the gp120 region were created by inverse PCR. In both assays, recombinant virus was obtained through homologous recombination in 293FT cells cotransfected with linearized deleted vector and the corresponding purified PCR product. After $60 \mathrm{~h}$ the supernatant was harvested and used to infect only U87-CXCR4 cells (INI assay) or both U87CXCR4 and U87-CCR5 cells (tropism assay). In the INI assay, after 7-10 days of culture in U87-CXCR4 cells the virus was titrated in TZM-BL cells and used to infect in triplicate TZM-BL cells in the 
presence of five-fold dilutions of RAL. In the tropism assay, after 7-10 days supernatants from both U87-CXCR4 and U87-CCR5 cells were collected and used to infect separately TZM-BL in triplicate. After $48 \mathrm{~h}$ virus activity was detected and titrated, allowing virus classification in CCR5 tropic, CXCR4 tropic or dualmixed tropic.

Results: During the first tests, the $\mathrm{IC}_{50}$ of RAL of wild-type virus was estimated to be $4 \mathrm{nM}$, while the $\mathrm{IC}_{50}$ of a recombinant virus harboring resistance mutations G140S and Q148H was $760 \mathrm{nM}$ (fold change 190), comparable to values reported in similar experiments with other phenotypic systems. Optimization of the different INI resistance assay steps is under way in order to define the most convenient procedure. A series of recombinant viruses incorporating the patient-derived gp120 have been obtained and tropism analysis is being performed.

Conclusions: The RVA developed in our laboratory can be successfully used to study susceptibility to RAL and other INI in vitro as well as to define HIV tropism phenotypically. Expected applications include both clinical use (e.g. unclear mutational patterns) and research studies, particularly characterization of investigational INI and generation of tropism phenotype-genotype correlation datasets to improve the accuracy of genotypic tropism assays.

\section{SC 21}

EAT VOLUME IS AN INDEPENDENT RISK FACTOR OF CARDIOVASCULAR DISEASE IN HIV-INFECTED PATIENTS

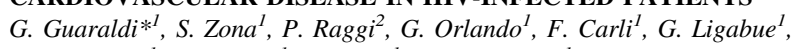
R. Scaglioni ${ }^{I}$, G. Besutti ${ }^{I}$, R. Rossi ${ }^{1}$, M.G. Modena ${ }^{I}$

${ }^{1}$ University of Modena and Reggio Emilia, Modena, Italy; ${ }^{2}$ Emory University, Atlanta, GA, USA

Background: Epicardial adipose tissue volume (EAT) is increased in HIV infected patients. EAT may contribute to coronary artery atherosclerosis via paracrine secretion of proinflammatory cytokines, playing a role in the development of cardiovascular disease (CVD). The aim of the study was to evaluate EAT associated factors and the relationship between EAT and occult CVD and predictors of EAT change over time.

Methods: Observational study of 876 consecutive HIV infected patients. Patients with previous CVD were excluded. EAT was measured in the same CT images obtained for CAC. Occult CVD was defined as CAC $>100$. Association between LD phenotypes and EAT was studied with non-parametrical tests. EAT associated factors were explored in a multivariable linear regression analysis. Multivariable logistic regression was used to evaluate association between CAC $>100$ and EAT. A subset of 192 patients were studied perspectively (median scan interval: 1 year, IQR 6;42 months) with repeated EAT measurements. Factors independently associated with EAT change predictors were explored in a multivariable generalized estimating equation regression model. Comparison between sensitivity and specificity of anthropometric measures, VAT and EAT changes were calculated with Roc curves.

Results: Patients' mean age was $47.2 \pm 8$ years, $68 \%$ were men. Men had greater EAT $\left(80 \mathrm{~cm}^{3}\right.$, IQR $\left.55 ; 103\right)$ than women $\left(61 \mathrm{~cm}^{3}, \mathrm{IQR}\right.$ $42 ; 82), \mathrm{p}<.001$. Figure 1 shows the association between LD phenotypes and EAT. Factors associated with EAT were: age $(\beta=0.6$, CI $0.2 ; 1.0)$, male gender $(\beta=6.6$, CI $0.5 ; 12.7)$, VAT $(\beta=0.12$, CI $0.08 ; 0.17)$, waist girth $(\beta=0.7$, CI $0.04 ; 1.3)$, liver fat $(\beta=-19.6$, CI $-32.6 ;-6.8)$, current CD4 $(\beta=0.6$, CI $0.1 ; 1.2)$, total cholesterol $(\beta=0.1$, CI $0.02 ; 0.15)$, cumulative exposure to ART $(\beta=0.05, \mathrm{CI}$ $0.00 ; 0.11)$. EAT volume was independently associated with CAC $>100$ (OR 1.10, CI $1.02 ; 1.20$ ). In the prospective substudy, factors associated with EAT change were CD4 reconstitution (defined as current-nadir delta CD4 cell count) $(\beta=0.5$, CI $0.1 ; 0.8)$, and time between CT scans ( $\beta=4.5$, CI $0.4 ; 8.7)$. EAT change had the closest association with CAC score $>100(\mathrm{p}=.047)$ (Fig. 2).

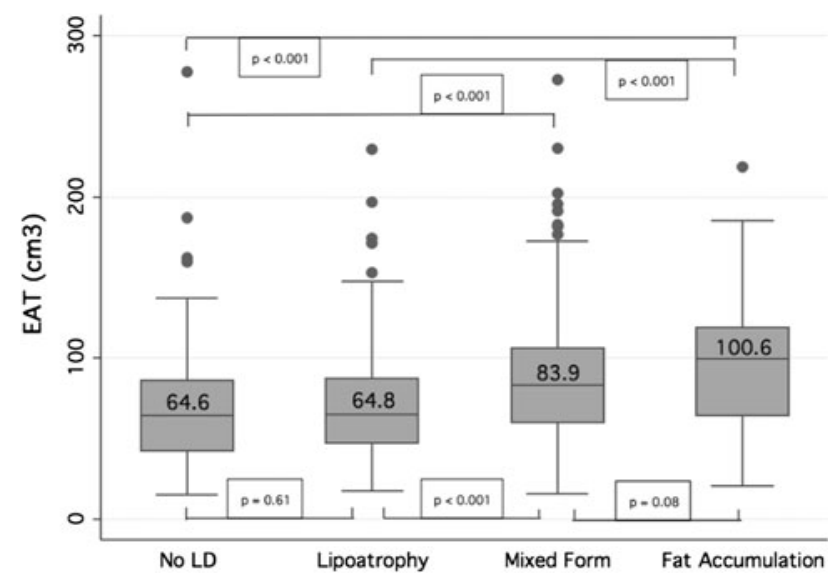

Fig. 1

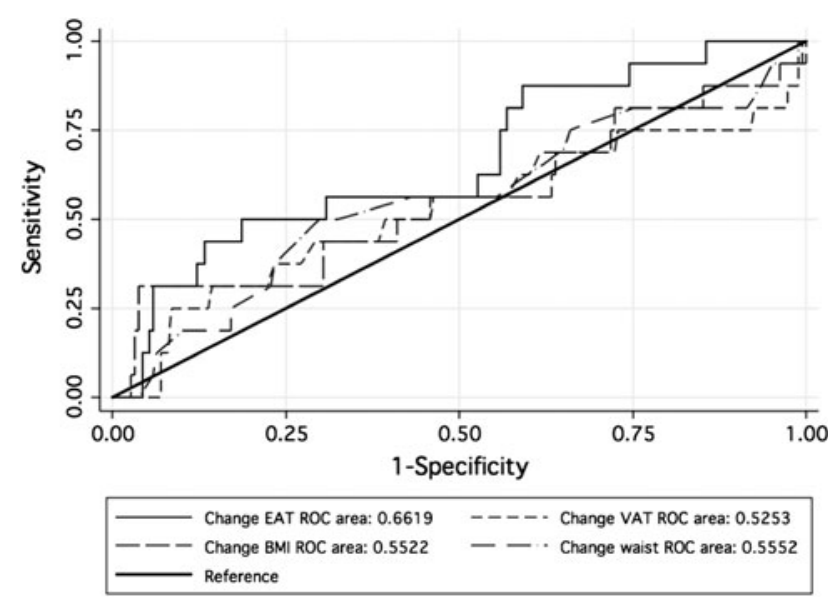

Fig. 2

Conclusions: This study shows an association between EAT and central fat accumulation and mixed form LD phenotypes. HIV specific and traditional factors, including male gender, were associated with EAT. CD4 increase from nadir was associated with EAT progression suggesting a possible influence of adiposity-related inflammation on atherosclerosis.

\section{SC 22}

OSTEOPROTEGERIN AS A BIOMARKER OF SUBCLINICAL ATHEROSCLEROSIS IN HIV-INFECTED MAN

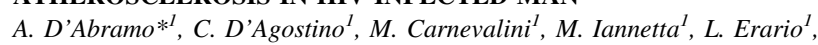

F. Cristofano ${ }^{I}$, V. Vullo ${ }^{I}$

"Sapienza" Università di Roma, Rome, Italy

Background: HIV-infected patients have an accelerated atherosclerosis. Currently atherosclerosis is considered an inflammatory disease. Several risk factors play an important role in the progression of atherosclerosis; metabolic disorders, a direct effects of protease inhibitors (PIs) as well as a primary impact of HIV infection result in vascular inflammation. Osteoprotegerin (OPG), a soluble member of TNF receptor superfamily, is known been implicated in bone remodelling blocking osteoclastogenesis, as well as in atherosclerotic progression, vascular calcification and vascular inflammation. The aim of our study was to evaluate the relationship between the extent 
of coronary atherosclerosis, measured by CT coronary scanner, osteoprotegerin plasma levels and other established risk factors.

Patients and methods: Population included 39 HIV infected man (age $53 \pm 9 \mathrm{y} / \mathrm{o}$ ), exposed to PIs successfully since at least 12 months, HIV/RNA $<50 \mathrm{cp} / \mathrm{ml}$, CD4 $433 \pm 248 \mathrm{mmc}$, Framingham score $<10$ and $21 \mathrm{HIV}$ negative man (age $58 \pm 8$ y/o) matched for traditional cardiovascular risk factors. Sixty-four slice CT coronary angiography was performed to determine coronary stenosis and c-IMT, measured ultrasonically. Historical and current immunevirologic parameters, metabolic criteria, blood pressure measurement, smoking status and family history of CAD. OPG and RANKL plasma levels were measured by an enzyme-linked-immunosorbent assay.

Results: OPG plasma levels were significantly lower in HIV+ patients than the controls $(5.7 \pm 2.64 \mathrm{pmol} / \mathrm{l} \mathrm{HIV}+$ vs. $8.3 \pm 4.6 \mathrm{pmol} / 1$ HIV-), ( $<<0.05)$. In our study we found no difference in plasma RANKL between two groups $(0.34 \pm 0.09 \mathrm{HIV}+$ vs. $0.38 \pm 0.14)$. Seventeen HIV+ patients (43.6\%) had no evidence of coronary stenosis; $7(17.9 \%)$ less than $50 \%$ stenosis and $15(38.5 \%)$ greater than $50 \%$ stenosis. Regarding c-IMT the values were: $1.1 \pm 0.25 \mathrm{~mm}$ $\mathrm{HIV}+$ vs. $0.601 \pm 0.04 \mathrm{~mm} \mathrm{HIV}$-patients. We found a negative correlation between OPG plasma level and c-IMT. As for HIV+ patients there was an inverse correlation between OPG values and greater atherosclerosis: $6.8 \pm 3.3 \mathrm{pmol} / 1$ (no stenosis) vs. $5.2 \pm 1.5 \mathrm{pmol} / \mathrm{l}$ (stenosis $<50 \%$ ) vs. $4.3 \pm 1.5 \mathrm{pmol} / \mathrm{l}$ (stenosis $>50 \%)(\mathrm{p}<0.05)$. Furthermore there is a negative correlation between cholesterol (HDL) and coronary plaque extension and a negative correlation between plasma OPG level and cholesterol (LDL) $\mathrm{p}=0.04$. We found an higher prevalence of CAD in HIV+ patients with lower CD4 nadir $(\mathrm{p}<0.05)$ and lower plasma concentration of OPG. No correlation between OPG, CAD and current CD4 and/or HIV/RNA.

Conclusion: In addition to the study of traditional $\mathrm{CV}$ risk factor, we focused the evidence regarding the role of OPG that could be considered a potential biomarker of silent atherosclerosis and/or a protective factor against the development of atheromasic plaque.

\section{SC 23}

LEFLUNOMIDE PROTECTS AGAINST DYSLIPIDEMIA DEVELOPMENT INDUCED BY RITONAVIR IN MICE

F. Baldelli ${ }^{1}$, A. Mencarelli ${ }^{2}$, B. Renga ${ }^{2}$, C. D'Amore ${ }^{2}$, S. Cipriani ${ }^{2}$, E. Schiaroli ${ }^{*}$, L. Bernini ${ }^{1}$, D. Francisci ${ }^{I}$, S. Fiorucci ${ }^{2}$

${ }^{I}$ Clinica di Malattie Infettive, Perugia, Italy; ${ }^{2}$ Dipartimento di Medicina

Clinica e Sperimentale, Perugia, Italy

Background: Treatment of HIV-infected patients with HIV Protease inhibitors (PIs) causes a dyslipidemia contributing to the development of cardiovascular diseases. Pregnane $\mathrm{X}$ receptor (PXR) is a nuclear hormone receptor activated by a diverse array of endogenous hormones and several studies indicated that PXR is involved in liver steatosis and dyslipidemia. Ritonavir is a human PXR agonist, as demonstrated by in vitro experiments. Short-term administration of leflunomide, a human PXR antagonist, to HIV-1 infected persons not receiving antiretroviral therapy effectively decreases $\mathrm{T}$ cell turnover and activation.

Aim: To investigate the effects of leflunomide on dyslipidemia induced by ritonavir in mice.

Methods: Eight weeks old C57/B16 wild type (WT) and "humanized" hPXR transgenic mice were randomized into three groups ( $N=6-8 /$ group): group 1 , no treatment; group 2, intraperitoneally administration of ritonavir ( $5 \mathrm{mg} / \mathrm{kg} /$ day $)$ alone and, group 3 , in combination with leflunomide $(40 \mathrm{mg} / \mathrm{kg} / \mathrm{day}$ by gavage $)$. All drugs were administered for 12 days. At the end of the experiment animals fasted $12 \mathrm{~h}$, received the latest administration and $4 \mathrm{~h}$ later were sacrificed.

Results: We found that 12 days of ritonavir administration in wild type (WT) and hPXR mice increases plasma triacylglycerols, cholesterol and LDL. This effect was abrogated by co-treating WT mice with leflunomide $(\mathrm{P}<0.05)$. In $\mathrm{hPXR}$ mice leflunomide revertes the hypertriglyceridemia induced by ritonavir $(\mathrm{P}<0.05)$, but has no effect on plasmatic cholesterol levels. Moreover this experiment demonstrates that treating WT mice with this PI has no effect on liver weight and liver/body weight ratio $(\mathrm{P}>0.05)$, whereas increases significantly liver triacylglycerols $(\mathrm{P}<0.05)$; this effect is reverted by leflunomide co-treatment $(\mathrm{P}<0.05)$. In WT mice ritonavir causes liver steatosis, induction of the liver expression of genes involved in PXR mediated triacylglycerols synthesis, and increases the triacylglycerol storage, such as the fatty acid elongation (FAE) and StearoylCoA desaturase 1 (SCD-1) $(\mathrm{P}<0.05)$. Leflunomide reduces the ritonavir induction of FAE $(\mathrm{P}<0.05)$, but in contrast increases SCD-1 mRNA levels $(\mathrm{P}<0.05)$ and in addition induces a prosteatogenic gene such as CD36 $(\mathrm{P}<0.05)$. In WT mice CYP3A11, a canonical PXR target gene, is induced by all treatments. In hPXR mice ritonavir induces alone SCD-1 gene and reduces CD36 expression $(\mathrm{P}<0.05)$. Leflunomide co-treatment in hPXR mice induced CYP3A11 and CD36 expression $(\mathrm{P}<0.05)$.

Conclusions and implications: We found that ritonavir administration to WT mice induces dyslipidemia and liver steatosis and these effects are antagonized by leflunomide. These data suggest that leflunomide might be a novel therapeutic target for preventing/treating lipid metabolism abnormalities caused by ritonavir.

\section{SC 25 \\ CIRCULATING MARKERS OF ENDOTHELIAL DISFUNCTION, FLOW-MEDIATED VASODILATION AND CIRCULATING ENDOTHELIAL CELLS IN HIV INFECTION: EFFECT OF ABACAVIR VERSUS TENOFOVIR

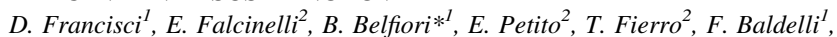 P. Gresele ${ }^{2}$ \\ ${ }^{I}$ Division of Infectious Diseases, Department of Experimental Medicine, \\ University of Perugia, Perugia, Italy; ${ }^{2}$ Division of Internal and Cardiovascular Medicine, Department of Internal Medicine, University of Perugia, Perugia, Italy}

Purpose: Ischemic cardiovascular events represent an increasingly frequent complication of long-lasting human immunodeficiency virus (HIV) infection and have been attributed either to the infection itself or to highly active antiretroviral therapy (HAART). Endothelial dysfunction is a precocious marker of atherosclerosis. Abacavir use has been associated with an increased risk of ischemic cardiovascular events in several cohort studies, but the pathogenic mechanisms are still unknown. Our aim was to assess whether patients with chronic HIV-infection present endothelial dysfunction and if this is the consequence of infection or of HAART, comparing patients treated with abacavir (ABC) or tenofovir (TDF).

Methods: In a retrospective, case-control study, the time course of soluble vascular cell adhesion molecule-1 (sVCAM-1) and monocyte chemoattractant protein-1 (MCP-1) was examined in 62 HIV-infected patients, before starting HAART and after 6-12 months of therapy with either $\mathrm{ABC}(\mathrm{n}=31)$ or TDF $(\mathrm{n}=31)$. Data were compared with those from 20 untreated HIV-infected patients at diagnosis and after 6 months and 10 healthy matched controls. Soluble endothelial activation markers were measured in plasma by flow cytometry using a bead-based assay. Of the above patients, 21 (11 ABC and 10 TDF) were recalled after a further 28-34 months for the measurement of flow mediated vasodilation (FMD) and circulating endothelial cells (CECs). FMD was measured using the Endo-PAT2000 system, a simple, noninvasive, diagnostic test for endothelial function; CECs were identified by CD45+, CD31+, CD146+ and quantified by flow cytometry. 
Results: sVCAM-1 and MCP-1 were significantly higher in HIVinfected patients at diagnosis than in healthy controls. During follow-up, sVCAM-1 and MCP-1 tended to decrease, without significant differences between $\mathrm{ABC}$ and TDF. In untreated HIV patients significantly increased plasma markers of endothelial dysfunction were confirmed at diagnosis with no changes upon followup. Overall, FMD was reduced by $21 \%$ (p < $<.05$ ) in HIV-infected patients as compared with healthy controls, with no difference between $\mathrm{ABC}(1.32 \pm 0.13)$ and TDF $(1.28 \pm 0.23, \mathrm{p}=\mathrm{ns})$. ABCtreated patients had significantly higher ECs when compared to controls $(16.8 \pm 8.1 / ? 1$ vs. $0.34 \pm 0.08 / ? 1, \mathrm{p}<0.05)$ and tendentially higher as compared with TFD-treated patients $(16.8 \pm 8.1 / ? 1$ vs. $9.3 \pm 3.8 / ? 1, \mathrm{p}=\mathrm{ns})$. Moreover, in the $\mathrm{ABC}$ group, but not in the TDF group, CECs were strongly inverse-correlated with FMD $\left(\mathrm{r}^{2}=0.81, \mathrm{p}=0.0001\right)$.

Conclusions: Our data confirm that HIV-infected patients have an impaired endothelial function and that antiretroviral treatment does not worsen it, although $\mathrm{ABC}$-treated patients tend to have a worsen endothelial function on follow up. More data need to be collected for CECs and FMD evaluation.

\section{SC 26}

BONE TURN-OVER MARKERS IN HIV POSITIVE PATIENTS AFTER HAART: TENOFOVIR (TDF) + EMTRICITABINE

(FTC) + ATAZANAVIR/RITONAVIR (ATV/R)-VS.-

TDF + FTC + EFAVIRENZ (EFV): RESULTS OF INCA TRIAL

E. Focä ${ }^{\prime}$, D. Motta ${ }^{I}$, M. Borderi ${ }^{2}$, I. Izzo ${ }^{I}$, A. Calabresi ${ }^{I}$, R. Bellagamba ${ }^{3}$, R. Fezza ${ }^{3}$, P. Narciso ${ }^{3}$, L. Sighinolfi, P. Maggi ${ }^{5}$, M. Mendeni ${ }^{1}$, G. Carosi ${ }^{1}$, D. Gotti ${ }^{1}$, A. Clo ${ }^{6}$, S. Morini ${ }^{6}$, B.M. Cesana ${ }^{7}$, E. Quiros-Roldan ${ }^{1}$, C. Torti ${ }^{1}$ ${ }^{I}$ Infectious Diseases Department, University of Brescia, Brescia, Italy;

${ }^{2}$ Infectious Diseases Institute, University of Bologna, Bologna, Italy; ${ }^{3}$ National Institute of Infectious Diseases, Rome, Italy; ${ }^{4}$ Infectious Diseases Department, S. Anna Hospital, Ferrara, Italy; ${ }_{5}^{5}$ Infectious Diseases Institute, Policlinico di Bari, Bari, Italy; ${ }^{6}$ Microbiology Section, University of Bologna, Bologna, Italy; ${ }^{7}$ Institute of Statistics in Medicine, University of Brescia, Brescia, Italy

Background: The aim of this study was to evaluate the trend in bone turnover markers, vitamin D3, and parathormone (PTH) after two standard antiretroviral regimens.

Methods: This study is a sub-study of the INCA trial, a randomized, multicentre study conducted in antiretroviral naïve patients who were randomized to TDF + FTC + ATV/r or EFV. Patients were assessed at baseline (i.e., before antiretroviral treatment), after 6 months, and after 1 year follow-up. The following markers were analyzed: vitamin $\mathrm{D}$, parathormone $(\mathrm{PTH})$, bone resorption markers (i.e., osteoprotegerin, OPG; carboxy-terminal collagen crosslinks, CTX; receptor activator of nuclear factor K-B ligand, RANK-L) and a bone formation marker (osteocalcin). Repeated measurement analysis of variance (ANOVA) was used, with data input performed according with last observation carried forward method. The overall time-variation of the markers in the two arms was evaluated by mixedfactorial random-coefficient general-linear-model.

Results: In this substudy, 75 patients were included: 42 in the ATV/r arm and 33 in the EFV arm; 61 (81.3\%) were males; mean age was 41.2 (SD 11.9) years; mean CD4+ was 274 (SD 128) cells $/ \mathrm{mm}^{3}$. No significant differences were found between the two arms at baseline. The overall trends were increasing for PTH values $(\mathrm{p}=0.0002)$, osteocalcin $(\mathrm{p}<0.0001)$, OPG $(\mathrm{p}=0.0432)$, and CTX $(\mathrm{p}<$ 0.0001). No significant decreases of vitamin D3 levels were found. Moreover, no significant differences for any markers between the two arms were found throughout the follow-up, except for CTX that decreased more in the EFV arm than in the ATV/r arm $(\mathrm{p}=0.0146)$. RANK-L was undetectable $(<0.0063 \mathrm{pg} / \mathrm{ml})$ in most patients throughout the study.

\begin{tabular}{|c|c|c|c|c|c|}
\hline \multirow[t]{2}{*}{ Marker } & \multicolumn{3}{|l|}{ Mean (SD) } & \multirow{2}{*}{$\begin{array}{l}\text { Overall } \\
\text { trend } \mathrm{p}\end{array}$} & \multirow{2}{*}{$\begin{array}{l}\text { Time- } \\
\text { arm p }\end{array}$} \\
\hline & Baseline & Week 24 & Week 48 & & \\
\hline \multicolumn{6}{|c|}{ Vitamin D3 (ng/ml) } \\
\hline Arm 1 & $17.19(8.37)$ & $17.72(9.21)$ & $16.95(5.87)$ & & \\
\hline Arm 2 & $\begin{array}{l}20.76 \\
\quad(11.54)\end{array}$ & $17.62(8.27)$ & $\begin{array}{l}17.68 \\
\quad(11.03)\end{array}$ & 0.2581 & 0.1969 \\
\hline \multicolumn{6}{|c|}{ PTH (pg/ml) } \\
\hline Arm 1 & $\begin{array}{l}30.75 \\
\quad(14.00)\end{array}$ & $\begin{array}{l}38.19 \\
\quad(18.02)\end{array}$ & $\begin{array}{l}45.37 \\
\quad(26.53)\end{array}$ & & \\
\hline Arm 2 & $\begin{array}{l}31.43 \\
\quad(22.50)\end{array}$ & $\begin{array}{l}42.46 \\
\quad(26.05)\end{array}$ & $\begin{array}{l}47.04 \\
\quad(38.61)\end{array}$ & 0.0002 & 0.8757 \\
\hline \multicolumn{6}{|c|}{ Osteocalcin (ng/ml) } \\
\hline Arm 1 & $\begin{array}{l}21.11 \\
\quad(11.55)\end{array}$ & $\begin{array}{l}38.23 \\
\quad(10.40)\end{array}$ & $\begin{array}{l}42.70 \\
\quad(14.73)\end{array}$ & & \\
\hline Arm 2 & $18.26(8.11)$ & $\begin{array}{l}32.74 \\
\quad(12.50)\end{array}$ & $\begin{array}{l}33.47 \\
\quad(12.50)\end{array}$ & $<0.0001$ & 0.1352 \\
\hline \multicolumn{6}{|c|}{ OPG (ng/ml) } \\
\hline Arm 1 & $0.84(0.42)$ & $0.87(0.32)$ & $0.93(0.40)$ & & \\
\hline Arm 2 & $0.82(0.39)$ & $0.91(0.37)$ & $0.90(0.33)$ & 0.0432 & 0.6323 \\
\hline \multicolumn{6}{|c|}{ CTX (ng/ml) } \\
\hline Arm 1 & $0.45(0.28)$ & $0.92(0.34)$ & $0.87(0.35)$ & & \\
\hline Arm 2 & $0.40(0.24)$ & $0.71(0.36)$ & $0.62(0.27)$ & $<0.0001$ & 0.0146 \\
\hline
\end{tabular}

Conclusion: These data confirm that antiretroviral therapy has a impact (either direct or mediated by its viro-immunological activity) on bone turn-over. Although this effect was more evident after 6 months, it persisted up to 1 year without any major differences between the two arms. Thus, TDF + ATV/r did not appear to alter the bone metabolism more than TDF + EFV co-administration.

SC 27

SUSTAINED VIROLOGICAL RESPONSE (SVR) TO ANTI-HEPATITIS C (HCV) THERAPY IS ASSOCIATED WITH A SIGNIFICANT INCREASE OF TRIGLYCERIDE (TGD) AND TOTAL CHOLESTEROL (TC) PLASMA LEVELS

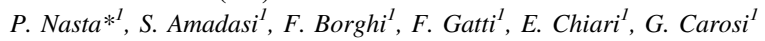

${ }^{I}$ Istituto di Malattie Infettive e Tropicali, Brescia, Italy

Background: total cholesterol (TC) and low-density lipoprotein (LDL) plasma levels are described to be lower in patients with chronic hepatitis $\mathrm{C}(\mathrm{CHC})$ because of the impaired hepatic lipids metabolism. We aimed to evaluate the lipid variation in $\mathrm{HIV} / \mathrm{HCV}$ coinfected patients (pts) with or without SVR after a peginterferon/ ribavirin (PegIFN/RBV) standard treatment.

Methods: all consecutive HIV/HCV coinfected pts who started PegIFN/RBV have been analyzed. SVR has been defined as negative HCV-RNA at 24 weeks post treatment follow up, by qualitative PCR assay (COBAS 2.0). Serum lipids parameters were measured at fasting in all pts at baseline (T0), and 12 months of follow up posttreatment (FU). Mann-Whitney and Fisher's test have been used to compare the variation of TRG and TC from T0 to FU in pts with or without SVR (SVR+ vs. SVR-). A multivariate logistic regression has been used to evaluate the association between the TC or TRG increase $>20 \%$ with the following baseline variables: gender, age, $\mathrm{HCV}$ genotype, TGC $>150 \mathrm{mg} / \mathrm{dl}, \mathrm{TC}>200 \mathrm{mg} / \mathrm{dl}$, insulin resistance, protease inhibitor (PIs) including antiretroviral regimens, SVR achievement.

Results: 107 HIV-HCV pts were enrolled: $84.1 \%$ males, 53 (49.5\%) infected by HCV genotype $1-4 ; 93.5 \%$ on HAART, $75.2 \%$ PIs based. 
The mean age $42.9(+5.4)$ years, TC $159(+40.4) \mathrm{mg} / \mathrm{dl}$, TRG 143.4 (+87) $\mathrm{mg} / \mathrm{dl}$, high density lipoprotein (HDL) $43.8(+13.4) \mathrm{mg} / \mathrm{dl} . \mathrm{SVR}$ was obtained in 47/100 (47\%). At FU, TC and TRG increased in SVR + [TC: from $159+41.2$ to $193+36.7 \mathrm{mg} / \mathrm{dl}$ (p 0.04); TRG: $130+91.6$ to $165.6+92.6 \mathrm{mg} / \mathrm{dl}(\mathrm{p} 0,01)]$; at the contrary remained unchanged in SVR-. HDL did not change significantly from BL to FU in SVR+ and SVR-. A $>20 \%$ of TC or TRG increase has been detected in 21/47 (44.2\%) SVR+ vs. 8/53 SVR- (15.1\%) [4.4 $(1.7-12.1)$ p 0.001$]$ and in 26/47 (55.3\%) SVR+ vs. 11/53 (20.7\%) SVR - [4.9 (2.0-12.1) $\mathrm{p}<0.001]$, respectively. At multivariate analysis SVR was the only variable related with the $>20 \%$ increase of TC (AOR 5.5, CI 1.6-19.2, p 0.006) or TRG (AOR 3.7, CI 1.2-11.1, p 0.01$)$ at FU.

Conclusions: HIV/HCV coinfected subjects who reached SVR experienced the increase of TC and TRG plasma levels. This finding may contribute to better describe the hepatic lipid metabolism in HIV subjects with chronic hepatitis C.

\section{SC 28}

\section{LIVER STEATOSIS IN HIV/HCV COINFECTED AND HCV-} MONOINFECTED PATIENTS: AN HISTOLOGICAL COMPARISON C. Sagnelli* ${ }^{*, 2}$, C. Uberti-Foppa ${ }^{2}$, L. Galli ${ }^{2}$, G. Pasquale ${ }^{I}$, S. De Pascalis ${ }^{2}$, M. Fiore ${ }^{1}$, N. Coppola ${ }^{1}$, L. Albarello ${ }^{2}$, C. Doglioni ${ }^{2}$, A. Lazzarin ${ }^{2}$, E. Sagnelli ${ }^{l}$ ${ }^{I}$ Department of Public Medicine, Section of Infectious Diseases, Second University of Naples, Naples, Italy; ${ }^{2}$ Clinic of Infectious Diseases and Department of Pathology, Vita-Salute University, San Raffaele Scientific Institute, Milan, Italy

Aim: To identify differences in liver steatosis between HIV/HCV coinfection and HCV monoinfection.

Methods: Liver biopsies from 190 consecutive HIV/HCV coinfected (Group HIV/HCV) and 190 age and sex pair-matched HCV monoinfected patients (Group HVC) were analyzed for steatosis (score $1=1-10 \%$ of hepatocytes with fatty deposition, score $2=11-30 \%$; score $3=31-60 \%$; score $4=>60 \%$ ). Included in the study were patients naïve for HCV treatment, aging 18 or more, HBsAg negative and with an alcohol intake lower than $40 \mathrm{~g}$ daily for males and $30 \mathrm{~g}$ daily for females. To evaluate the influence of the age on the degree of liver steatosis patients were distributed in four age classes: 18-30 years, 31-40, 41-50, more than 50.

Results: HIV/HCV coinfected patients showed a good immunological condition $(\mathrm{CD} 4=542+192.51)$ and compared with those $\mathrm{HCV}$ monoinfected more frequently showed HCV-genotype 3 (53.7 vs. $5.5 \%, \mathrm{p}<0.001)$; most patients with HCV genotype three were drug addicts. Severe steatosis (score 3 or 4 ) was more frequent in Group HIV/HCV than in Group HCV (47 vs, $21.1 \%$, p < 0.005) with statistical significance also in single age classes 18-31 $(\mathrm{p}=0.001)$, $31-40(\mathrm{p}=0.007)$ and $41-50(\mathrm{p}=0.00003)$. The frequency of patients with severe steatosis did not increase with the increase of the age, neither in Group HIV/HCV nor in Group HCV. In Group HIV/ HCV severe steatosis was more frequent in patients with $\mathrm{HCV}$ genotype 3 than in those with other genotypes (56.4 vs. $3.1 \%$, $\mathrm{p}=0.02$ ); analyzed in relation with the HAART regimen administered at the time of liver biopsy no difference in the frequency of severe steatosis was observed between patients receiving a PI HAART based, PI + D4T HAART, NNRTI, HAART including only D4T or left untreated. Other finding in both Group HIV/HCV and $\mathrm{HCV}$ was the absence of correlation between the degree of liver steatosis and that of fibrosis and necroinflammation, nor between liver steatosis and aminotransferasis, CD4+ cell count, nadir of CD4+, HIV and HCV viral load.

Conclusion: Severe liver steatosis is a frequent event in $\mathrm{HIV} / \mathrm{HCV}$ coinfected patients dining alcool abuse, related to the presence of HCV genotype 3 in drug addicts, but no correlation was observed with HAART administration.
SC 30

CHARACTERISTICS OF ANTI-HBV TREATMENT IN A COHORT OF ITALIAN HBV-INFECTED PATIENTS IN RELATION TO HIVINFECTION STATUS

G. Antonucci ${ }^{\prime}{ }^{1}$, E. Girardi ${ }^{1}$, C. Angeletti ${ }^{1}$, F. Mazzotta ${ }^{2}$, E. Sagnelli ${ }^{3}$, SIMIT Study Group ${ }^{4}$

${ }^{I}$ INMI L. Spallanzani, Rome, Italy; ${ }^{2}$ U.O. di Malattie Infettive Ospedale S. M. Annunziata, Florence, Italy; ${ }^{3}$ Clinica Malattie Infettive Seconda Universita, Naples, Italy; ${ }^{4}$ SIMIT

Background: In recent years, new factors are modifying the clinical features of HBV infection: (1) availability of sensitive techniques for HBV DNA detection; (2) increasing immigration from endemic areas; (3) increasing number of anti-HBV drugs. Based on these facts, a multicenter cross-sectional survey was carried out in 2008 by Italian Society of Infectious and Tropical Diseases (SIMIT) to provide an accurate picture of HBsAg+ chronic liver disease in Italy. The aim of the present analysis is to describe characteristics of current anti-HBV treatment in relation to HIV-infection status.

Methods: The study was performed in 74 Italian Infectious Diseases Units. All the 3,760 adult HBsAg+ patients observed consecutively in participating units from March 1, 2008 to September 30, 2008 were enrolled.

Results: Among patients included in the analysis, 427 (13.7\%) were $\mathrm{HIV}+, 303(9.9 \%)$ were cirrhotic, and $1,743(46.4 \%)$ on treatment. At univariable analysis $\mathrm{HIV}+$ patients were significantly more likely to be male (75.9 vs. $55.9 \%)$, born in Italy ( 82.9 vs. $70.1 \%), \mathrm{HBeAg}+$ ( 35.1 vs. $16.9 \%), \mathrm{HCV}+(32.4$ vs. $5 \%), \mathrm{HDV}+(19.5$ vs. $3.9 \%)$ than HIV - patients. Conversely, they were less likely to be alcohol abstainers (27.1 vs. $44.5 \%$ ) and to have had a diagnosis of HBsAg+ since $<2$ years $(11.2$ vs. $20.9 \%)$. Finally, HIV+ patients were younger $(44.6+9.1$ vs. $47.3+14.8$ years, $\mathrm{p}<0.001)$. At multivariable analysis HIV positivity was independently associated with the chance of treatment (AOR: 6.7, 95\% CI 4.4-10.2, p < 0.001). Among the $334 \mathrm{HIV}+$ patients treated, 58\% assumed TDF + LAM/FTC, $28 \%$ LAM/FTC as monotherapy, 5\% TDF, and 1\% Peg-IFN. Distribution of anti-HBV drugs among the 1,112 HIV-treated patients was quite different: $29 \%$ took LAM + ADV, $21 \%$ ETV as monotherapy, $20 \%$ LAM, Peg-IFN 16\%, ADV 11\%, and TDF 3\%.

Conclusions: Our data stress that $\mathrm{HIV} / \mathrm{HBV}+$ patients are more frequently treated than HBV monoinfected patients. Given that antiretroviral regimens may include drugs with activity against HBV, in $\mathrm{HIV} / \mathrm{HBV}+$ patients anti-HBV treatment is mainly given within cART regimen irrespective of the severity of HBV disease.

\section{SC 31}

INCIDENCE OF C. TRACHOMATIS GENITAL INFECTIONS IN A COHORT OF HIV INFECTED WOMEN

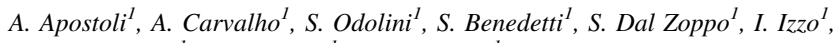
V. Bergamaschi ${ }^{1}$, S. Caligaris $^{1}$, A. Matteelli $^{* 1}$

${ }^{I}$ Department of Infectious Diseases, Spedali Civili, Brescia, Brescia, Italy

Introduction: Chlamydia trachomatis genital infection is the most frequently reported bacterial sexually transmitted infection (STI) in industrialized countries. In Italy, there are few studies regarding the prevalence and incidence of chlamydial infection among HIV-seropositive women.

Objectives: we aimed at determining the incidence and prevalence of C. trachomatis genital infection in a cohort of HIV infected women. We evaluated the possible association of biological and behavioural risk factors with chlamydial infection.

Methods: a prospective cohort study of HIV-infected women (DOSET-HIV), carried out from July 2003 to October 2009 at the STI centre of the Institute of Infections and Tropical Diseases at the University of Brescia was done. All women attending the HIV outpatient department were invited to participate if had at least 18 years 
of age and were willing to provide informed consent. Longitudinal assessments were scheduled every 6-12 months. At enrolment, women were asked about sociodemographic characteristics, lifetime obstetrical and gynecological history and risk behavior for HIV infection. CD4+ T-lymphocytes and HIV-RNA were measured at 3 month intervals. At each visit thorough physical and gynecological examinations were performed and microbiological samples were collected from the cervical sites for screening of $C$. trachomatis infection (BD ProebeTecTM ET C. trachomatis trachomatis).

Results: 126 HIV-infected women adhered to the study. The median age was 37 years (range 20-46); 79\% (100/126) were Italians and $59 \%(74 / 126)$ acquired the infection by unprotected heterosexual intercourse. Fifty-four percent of women (50/92) reported an irregular use of condom during sexual intercourse. The prevalence of $C$. trachomatis was 6.3\% (8/126). During follow up, 110 (87.3\%) HIVseropositive women were evaluated at least once, for a median time of observation of 3.56 years (range 6 months- 6 years). A total of 9 new cases of $C$. trachomatis genital infection was diagnosed, for a incidence rate of 2.45 cases/100 persons-year (95\% CI 0.95-3.94 cases/ 100 persons-year). In the logistic regression analysis, increased age at the first sexual intercourse (OR 1.44; 95\% CI 1.08-2.0; $\mathrm{p}=0.02$ ), higher CD4+ T-lymphocyte count (OR 0.99 ; 95\% CI $0.98-0.99$; $\mathrm{p}=0.04$ ) and longer follow-up (OR 1.86; 95\% CI 1.09-3.59; $\mathrm{p}=0.04)$ were independently associated with the risk of $C$. trachomatis genital infection.

Conclusion: a high prevalence and incidence of $C$. trachomatis genital infection among HIV-infected women were found. A screening program in this population is justified by this evidence. The risk of $C$. trachomatis infection is reduced in HIV seropositive women with higher CD4+ T cell count but increases with older age at sexual debut and with length of follow-up.

\section{SC 32 PORPHYRIA CUTANEA TARDA IN TWO ARV-SUPPRESSED PATIENTS WITH HIV/HCV COINFECTION

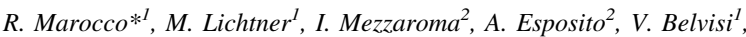 H. Martini ${ }^{2}$, P. Giovangrossi ${ }^{3}$, F. Equitani ${ }^{3}$, C.M. Mastroianni ${ }^{1}$ \\ ${ }^{1}$ Sapienza Università di Roma, polo pontino, Rome, Italy; ${ }^{2}$ Sapienza Università di Roma, Rome, Italy; ${ }^{3}$ Ospedale SM Goretti, Latina, Italy}

Cutaneous manifestations are frequently observed in patients with HIV infection. In pre-ART era most of them are related to opportunistic infection involving skin, but now with the continuing introduction of new antiviral drugs, they are primary caused by immune allergy. The correct diagnosis is often difficult and require histology. Here we present two cases of porphyria cutanea tarda (PCT) with skin fragility and bullous lesions in photo-exposed area in patients with HIV/HCV coinfection.

Case 1: a 46 years old woman with a long history of HIV infection (diagnosis in 1985, CDC:B3) and HCV chronic hepatitis. She was on ARV therapy since 1992 and she experienced several drugs including NRTI, NNRTI and boosted PI with virological suppression. She often self-stopped therapy due to skin manifestations. Regarding HCV hepatitis she was treated twice with IFN + RBV with virological relapse. In 2010 she stopped ARV and restarted it after 2 months with a new combination (CCR5 tropic virus was revealed): abacavir, 3TC and maraviroc. After 1 months she presented skin fragility and bullous skin lesions on her hand, interpreted us prurigo. She did not stop therapy this time because of very good immune virologic results (HIV-RNA undetectable, CD4+ 540). After 5 months skin lesions get worse and diffused to the face. An expert advice suggested $24 \mathrm{~h} /$ urinary and plasma porphyrin dosage that let us to diagnosis of Porphyria cutanea tarda. She started erythro-apheresis with a progressive decrease of urine and plasma porphyrins (from $5.13 \mathrm{mg} / 24 \mathrm{~h}$ to 1.98 and from $0.104 \mathrm{mg} / \mathrm{L}$ to 0.059 respectively).
Case 2: a 51 years old man with a long history of HIV infection (diagnosis in 1986, CDC: C3) and HCV chronic hepatitis. He was on ARV therapy since 1990 and he changed several regimens including NRTI, NNRTI and boosted PI for the occurence of adverse effects. In 2005 he started fosamprenavir, atazanavir, ritonavir and abacavir/ lamivudine with undetectable HIV-RNA and lymphocytes T CD4+ $380 / \mathrm{mmc}$. Regarding HCV hepatitis he was treated in 2009 with IFN + RBV with virological failure. After 3 months he showed recurrent bullous skin lesions on his upper limbs. After 1 year skin lesions get worse and the patient underwent cutaneous biopsy with diagnosis of Porphyria cutanea tarda type I. He started a cycle of phlebotomies with a decrease of urine porphyrins and resolution of symptoms.

In these two cases PCT was associated to a long HIV infection with viral control associated with $\mathrm{HCV}$ not advanced chronic infection. Although HCV infection seems to be the most important factors in determining the disease, also antiviral therapy could be implicated. Indeed the complex metabolic way of porphyrins accumulation could be inhibited by antiviral drugs increasing the cytochrome $\mathrm{P} 450$ activity or SA-heme-oxidation. Both phlebotomy and erythro-apheresis seem to be effective in controlling the diseases.

\section{SC 33 \\ MALIGNANT SYPHILIS CONFIRMED IN SKIN BIOPSY BY IMMUNOHISTOCHEMICAL STAINING IN AN HIV-INFECTED PATIENT \\ G.V. De Socio ${ }^{\prime}$, S. Simonetti ${ }^{2}$, A. Sgrelli ${ }^{\text {, E. Eecchini }}{ }^{\text {, M.V. Moretti }}{ }^{I}$, F. Baldelli ${ }^{l}$ \\ ${ }^{I}$ Clinica Di Malattie Infettive, Perugia; ${ }^{2}$ Clinica dermatologica, Perugia}

Introduction: Malignant syphilis is a rare disease. The histologic characteristics of lues maligna are similar to those of secondary syphilis; however, spirochetes are usually not found in the lesions. We demonstrates that appropriate Immunohistochemical (HIC) study with antitreponema antibodies can identify the etiologic agent in skin biopsy.

Case report: A 34-year-old man with HIV infection developed multiple atypical cutaneous ulcerations. The patient had a history of advanced HIV disease $\left(\mathrm{CD} 4+=80 / \mathrm{mm}^{3}\right)$, he voluntarily interrupted ART 16 months earlier. On clinical examination he had a temperature of $38^{\circ} \mathrm{C}$. He presented with facies leonine, diffuse large papules and plaques with erythematous-edematous circles with well demarcated borders; a number of lesions demonstrated central crusted ulceration. His right eye presented with a scleral nodule and keratitis. Other notable findings included generalized tender lymphadenopathy, and hepatosplenomegaly. Laboratory abnormalities included: leukopenia, and anemia. A dermatologist made a clinical diagnosis of ecthyma gangrenosum. Skin biopsy subsequently revealed an alternative diagnosis. Serological tests revealed a RPR of 1:32, with a positive IgM, a positive FTA test, and a TPPA titer of 1:5,120. Given the serologic test results for syphilis, and after considering the skin manifestations the patient received 24 million units of iv penicillin $\mathrm{G}$ per day, in addition to iv tigecycline. Topical tetracycline ointment and ocular antibiotics were also administered. The Jarisch-Herxheimer reaction occurred during the first $24 \mathrm{~h}$ of penicillin administration. Upon completion of the two-week treatment period all the lesions were healed with residual hypopigmentation and scarring. The skin histology exam revealed the presence of lymphocytes, histocytes and plasma cells. HIC staining for Treponema pallidum revealed a large number of spirochetes. These results were obtained towards completion of the course of therapy. At follow-up visits only disseminated dyschromic patches were evidenced. Serology after 4-9 months revealed RPR of 1:8 and 1:2, respectively, both with a negative IgM test.

Discussion: Clinical manifestations of secondary syphilis vary greatly, earning the epigram of "great imitator". After the advent of 
HIV infection, lues maligna has become more common. In our case the skin manifestations were erroneously considered clinically consistent with a possible nonspecific bacterial infection. The diagnosis was actually established through strongly positive serological tests, a severe Jarisch-Herxheimer reaction, an excellent response to antibiotic therapy and, most importantly, the positive HIC study with antitreponema antibodies, which represents a rarity in the literature. We emphasize the importance of lues maligna in the differential diagnosis of HIV-infected patients with diffuse ulceronodular lesions as well as the usefulness of histological investigations completed by HIC study.

\section{PP 01}

GENETIC DIVERSITY AT ENDOPLASMIC RETICULUM AMINOPEPTIDASES 2 (ERAP2) IS COUPLED WITH INNATE RESISTANCE TO HIV-1 INFECTION

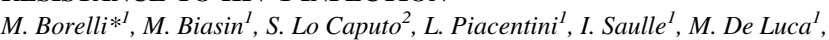
M. Sironi ${ }^{3}$, R. Cagliani ${ }^{3}$, D. Trabattoni ${ }^{1}$, F. Mazzotta $^{2}$, M. Clerici $^{1}$

${ }^{1}$ Università degli Studi di Milano, Milan, Italy; ${ }^{2}$ S.M. Annunziata Hospital,

Florence, Italy; ${ }^{3}$ IRCCS E. Medea, Lecco, Italy

Background: Analyses performed in subjects that despite multiple exposures to HIV never seroconvert or shown any sign of infection (HIV exposed seronegative individuals, HESN) revealed that this phenotype can be associated with the exclusive priming of protective T lymphocytes presumably within a favourable genetic setting. Thus, as the endoplasmic reticulum aminopeptidases type 2 (ERAP2) trims the peptides that will be loaded on MHC class I, shaping the quality of the peptides that will be presented to CD8+ T lymphocytes we investigated its role in resistance to HIV infection.

Materials and methods: SNP Analysis of the Asn392Lys (rs2549782) in ERAP2 variant was performed in an Italian population of HESN individuals and healthy controls. Furthermore, in both IFNg stimulated and HIV-infected PBMC isolated from subjects with a different ERAP2 haplotype we evaluated expression levels of mRNA specific for ERAP2 and the results were correlated with the levels of MHC class I molecules on CD45 cells and HIV p24 antigen respectively.

Results: The genotype distribution of rs 2549782 in ERAP2 is significantly different in the two cohorts $(\mathrm{P}=0.004)$, mainly as the result of an over-representation of Lys/Lys genotypes (haplotype A) in the ESN sample. ERAP2 expressed from haplotype B undergoes differential splicing encoding a mRNA containing an extended exon 10 , which results in a truncated ERAP2 protein. Interestingly, individuals homozygous for Haplotype A: (1) produced higher quantities of ERAP2 both in response to IFNg stimulation and HIV infection; (2) were less susceptible to HIV infection and, (3) had higher MHC class I molecules expression on primary lymphocytes.

Conclusions: These data suggest that ERAP2 variants confer resistance to HIV-1 infection possibly affecting MHC presentation and immune response.

\section{PP 03}

\section{HORMONAL STATUS AND NATURAL IMMUNITY IN HIV} INFECTED PATIENTS

R. Rossi ${ }^{*}$, G. La Martire ${ }^{I}$, M. Lichtner ${ }^{2}$, P. Massetti ${ }^{I}$, I. Sauzullo ${ }^{I}$,

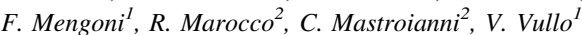

${ }^{I}$ Sapienza Università di Roma, Rome, Italy; ${ }^{2}$ Sapienza Università di Roma Polo Pontino, Rome, Italy

Background: Recent studies suggest that there are sex differences in the innate immune response to infection that could explain a different clinical course of HIV infection in women. We recently reported that men had higher levels of circulating mDCs compared to women in $\mathrm{HIV}+$ and HIV - subjects.

Objectives: To understand the role of sexual hormones on dendritic cells compartment, immune activation status and cytokines production.

Methods: Study population included 35 HIV infected patients and 35 healthy donors ( 15 women, 5 pregnant, 15 men). Circulating mDCs, pDCs, HLA-DR+/CD38+/CD4+ T or CD8+ T were assessed in whole blood samples using TruCOUNT assay. All patients were tested for estrogen, progesterone and testosterone plasma levels with ELISA kits. Blood was stimulated over-night with LPS, LPS+IFN-gamma and SN were tested for IP10, IFN-gamma, IL-12, IL-2 production with FlowCytomix Pro 2.4 (eBioscience).

Results: As we seen in our precedent study, women had lower levels of mDCs than men in HIV patients and in controls. We found a positive correlation between testosterone levels and mDCs count ( $\mathrm{p}=0.038$ in men group and $\mathrm{p}=0.002$ in women group). Also in HIV patients $\mathrm{mDC} /$ testosterone correlation was present. There were no significant differences in circulating $\mathrm{pDC}$ and $\mathrm{CD} 4$ count between men and women. Pregnant women showed higher levels of activated circulating CD4+ T lymphocytes (HLADR+CD38+) compared to non pregnant women $(\mathrm{p}=0.004)$. In pregnant women we found a positive correlation between progesterone levels and TNF-alpha production $(\mathrm{p}=0.0149)$. In both pregnant and non pregnant groups the immune activation level, in terms of CD4+HLADR+CD38+, had a positive correlation with oestrogen levels $(\mathrm{p}=0.01$ in non pregnant group and $\mathrm{p}=0.009$ in pregnant group) and estrogen levels also had a negative correlation with the IP10 level produced in response to LPS stimulation $(\mathrm{p}=0.0131)$. No significant correlations between estr, prog, testost levels and IP10, TNFalpha, IFN gamma production in all the other groups. Concerning IP10 production in response to LPS stimulation, we noticed that there were a significant difference between HIV - and HIV+ groups, HIV - people producing more IP10 (mean $4,383 \mathrm{pg} / \mathrm{mL})$ than $\mathrm{HIV}+$ people $(2,066 \mathrm{pg} / \mathrm{mL})$ with $\mathrm{p}=0.001$. As expected, in HIV+ group there was a higher IFNgamma production in response to LPS compared to HIV - group (244.16 vs. 0 ; $p=0.004$ ) Conclusions: The study suggests that innate immunity could play an important role in driving the differences in immune activation and disease progression in HIV-infected women and men. Our study also suggest that sexual hormones are in part responsible of immune differences between women and men and between pregnant and non pregnant women. These results could be a starting point for a more precise gender definition of immunity and a gender-specific analysis of HIV infection, opening also to the possibility of different therapeutic strategies

\section{PP 04}

DO PEOPLE VIROLOGICALLY SUPPRESSED ARE REALLY IN OPTIMAL CLINICAL CONDITIONS?

R. Murri ${ }^{1}$, A. Cingolani $^{l}$, A. De Luca ${ }^{I}$, S. Di Giambenedetto ${ }^{I}$, G. Marasca ${ }^{l}$, G. De Matteis ${ }^{I}$, M. Fabbiani ${ }^{I}$, E. Jacoboni ${ }^{1}$, I. Mastrorosa ${ }^{I}$, E. Tamburrini ${ }^{I}$

${ }^{1}$ Università Cattolica S Cuore, Rome, Italy

Objective: To describe physical and mental health, symptom burden and adherence behaviours in people with an optimally viro-immunological outcome.

Methods: cohort, monocenter, prospective study on people attending the Outpatient Service for HIV people of Catholic University of Rome and taking cART. A short questionnaire on adherence to drugs and health status was administered. Self-reported adherence was estimated on a 0-100 scale. A Symptom Score was built summing self-reported scores for each of 19 listed symptoms (range 0-76).

Results: 878 patients were enrolled the questionnaire, all with HIV RNA $<50 \mathrm{c} / \mathrm{ml} .33 .2 \%$ females, mean age 47.2 years (SD 8.3), IDU

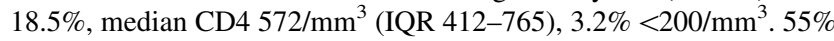
were taking PI and 33\% NNRTI. Mean SelfAdher was 80.8 (SD 17.4). 119 persons (14.9\%) reported having missed one dose of antiretrovirals during the week before the survey and $142(16.2 \%)$ 
reported an adherence $<60.23 .3 \%$ of people reported having discontinued drugs for more than $24 \mathrm{~h}$. Mean of PHS and MHS were 72.3 (SD 17.5) and 73.9 (SD 20.2). 4.5 and $8.7 \%$ had a PHS and MHS below 40. More than $5 \%$ of people reported fatigue, stomachache, diarrhea, abdominal bloating, mental confusion, insomnia, breath troubles, myalgias, pain at extremities, anxiety, depression, lipoaccumule, and sexual disturbances as "much" or "very much" during the month before. Symptom score was strongly correlated to selfreported adherence $(\mathrm{p}<0.001)$. Jaundice, nausea, diarrhea, abdominal bloating, mental confusion, myalgias and depression were independently correlated to adherence in a multivariable linear regression analysis (all $\mathrm{p}<0.05$ ).

Conclusions: Adherence deviation behaviours are frequent even in people with optimal viroimmunological outcome. Burden of symptoms in this population was high. Further analysis should investigate the predictive value of adherence deviation behaviours of viroimmunological failure during follow-up

\section{PP 05}

\section{THYMIC- AND BONE MARROW-CELLULAR RESERVOIRS HARBOR PHYLOGENETICALLY DISTINCT HIV STRAINS AND DIFFERENT RESISTANCE PROFILES}

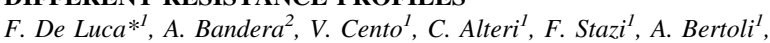
M. Montano ${ }^{I}$, A. Soria ${ }^{2}$, M. Andreoni ${ }^{I}$, C.F. Perno ${ }^{l}$, A. Gori ${ }^{2}$, F. Ceccherini Silberstein ${ }^{1}$

${ }^{1}$ University of Tor Vergata, Rome, Italy; ${ }^{2}$ "San Gerardo" Hospital University of Milan-Bicocca, Monza, Italy

Background: HIV-1 treatment can decrease plasma HIV-1 RNA to undetectable levels, yet integration of HIV-1 DNA in cellular reservoirs is the major obstacle for HIV-1 eradication. So far, few studies have evaluated the in vivo HIV-1 quasispecies presence within different cellular reservoirs. The aim of this study was to evaluate the quantity and genetic characteristics of integrated HIV-1 DNA in different cellular reservoirs of HAART-multiexperienced successfully treated patients.

Methods: Real time PCR quantification and genotypic analysis (PR/ RT and V3) of HIV-1 DNA was performed in peripheral blood mononucleated cells (PBMC), bone marrow cells (BMMC) and thymic cells of 3 HIV-1 B subtype infected patients. All patients were successfully treated at the time of the study (HIV-1 RNA $<50 \mathrm{cp} / \mathrm{ml}$, 383 cells $/ \mathrm{mm}^{3}$ mean CD4 cell count).

Results: HIV-1 DNA was detectable in PBMC of all patients (range $718-7,088 \mathrm{cp} / 106$ cells), and in BMMC of $2 / 3$ patients (133 and $6,184 \mathrm{cp} / 106$ cells). Thymic cells (CD4+CD8-, CD4+CD8+ and CD4-CD8+) had HIV-1 DNA below the detection threshold $(15 \mathrm{cp} /$ 106 cells), with the exception of CD4+CD8 - cells obtained from one patient (33 cp/106 cells). PR/RT and V3 sequencing from PBMC and BBMC was successful for all patients. PR/RT sequence from thymocytes was obtained for one patient (CD4+CD8 - cells), despite the low HIV-1 DNA level. All patients carried PR/RT resistance mutations (associated with previously failing treatments) in HIV-1 DNA of PBMC [mean number of drug resistance mutations (DRMs): 8 , range: 5-15]. The presence of drug resistance was confirmed in BMMC of $2 / 3$ patients, even if with a lower number of DRMs (mean: 4 ), and in the available PR/RT sequence from CD4+CD8 - cells (DRMs: 12). By analyzing V3 sequences, we found that $2 / 3$ patients had archived X4-tropic variants in both PBMC and BMMC. Phylogenesis and drug resistance analysis showed that $\mathrm{CD} 4+\mathrm{CD} 8$ - thymocytes harbored viral sequences more closely related to PBMC (genetic distance: $0.014+0.004$ ) than BMMC HIV-1 variants (genetic distance: $0.093+0.007)$, suggesting a gradual diversification and evolution among viral variants in different lymphoid reservoirs.

Conclusions: Our results demonstrate different patterns of viral evolution in cells from peripheral blood, thymus and bone marrow. In particular, the ability of these cells to harbor drug resistant HIV-1 strains, confirms their role to act as a long-lived reservoirs of drug resistance. Furthermore, the limited diversification of BMMC HIV-1 DNA in one patient indicates that bone marrow cells may represent a distinct and ancestral viral reservoir.

\section{PP 06}

HIV AVIDITY INDEX COMPARED TO OTHER VIROLOGICAL PARAMETERS IN RECENT AND ESTABLISHED HIV INFECTIONS: DIAGNOSTIC RELEVANCE

S. Faraoni ${ }^{1}$, M. Proia ${ }^{\prime}{ }^{1}$, M.G. Milia ${ }^{1}$, T. Allice ${ }^{1}$, G. Gregori ${ }^{1}$, E. Burdino ${ }^{l}$, T. Ruggiero ${ }^{1}$, A. Signorino ${ }^{1}$, C. Galli ${ }^{2}$, S. Bonora ${ }^{3}$, G. Orofino ${ }^{3}$, V. Ghisetti ${ }^{1}$ ${ }^{1}$ Laboratory of Microbiology and Virology, Amedeo di Savoia Hospital, Turin, Italy; ${ }^{2}$ Scientific Affairs, Abbott Diagnostic, Rome, Italy; ${ }^{3}$ Department of Infectious Diseases, Amedeo di Savoia Hospital and University of Torino, Turin, Italy

Objectives: To evaluate the patterns and diagnostic accuracy for the discrimination of recent from established infections by human immunodeficiency virus (HIV) of different serological parameters.

Methods: The study population included all patients newly diagnosed with HIV over 26 months (March 2008-April 2010). The diagnosis of acute, recent ( $<6$ months) or established ( $>6$ months) infection was based upon clinical criteria and/or the knowledge of the previous serostatus for HIV. On the first available serum specimen, the following serological tests were performed: (a) an automated 4th generation assay (ARCHITECT HIV Ag/Ab, Abbott Diagnostics), reporting both the positivity and the signal to cutoff $(\mathrm{S} / \mathrm{CO})$ ratio; (b) a confirmatory Western lot (New LAV BLOT I, BioRad, F), yielding a positive, indeterminate or negative result and also detailing the presence/absence of the different antigen bands; (c) the anti-HIV avidity index (AI), calculated as the ratio of the $\mathrm{S} / \mathrm{CO}$ rates (ARCHITECT HIV Ag/Ab) of two aliquots of serum diluted 1:10 in $1 \mathrm{M}$ guanidine hydrochloride or in PBS, respectively. Additionally, the HIV viral load has been determined on a significant percentage of patients by real-time PCR (COBAS AmpliPrep/Cobas TaqMan, HIV1 version 2.0 Roche, Diagnostics, Italy)

Results: We considered 365 patients, of whom 9 with an acute $(2.5 \%), 35$ with a recent $(9.6 \%)$ and 321 with an established infection (87.9\%). All cases were reactive by the ARCHITECT 4th generation assay, with median S/CO values of 36 in acute, 108 in recent and 430 in established infections. The percentage of WB positive cases increased from $11.1 \%$ in acute to $60.0 \%$ in recent and to $95.8 \%$ in established infections ( $p<0.001$ by Chi-square for linear trend); the most frequently reactive bands in all phases of infection were those against the env region antigens; of note, the positivity for gp41 increased according to the duration of infection from $0 \%$ in acute to $51.4 \%$ in recent and to $96.9 \%$ in established infection. An $\mathrm{AI}<0.80$ was found in $42 / 44$ acute and recent infections (95.4\%) and only in 4/321 (1.2\%) established infections. Considering as misclassified also the 25 results $(6.8 \%)$ that fell in the grey zone (AI $0.75-0.84)$, the AI accuracy was $92.1 \%$.

Conclusion: The AI is the most reliable serological parameter for the discrimination of recent HIV infections. The calculation of this index is easy and it is especially useful in surveillance programs, where it will allow an estimation of the incidence of HIV infection and the relative proportion of newly infected individuals according to different risk behaviours.

\section{PP 07}

HUMAN IMMUNODEFICIENCY VIRUS TYPE 1 VIRAL LOAD AND DRUG RESISTANCE ON DRIED BLOOD SPOTS

B. Matteoli ${ }^{I}$, A. Scaccino ${ }^{I}$, L. Bontempo ${ }^{I}$, L. Ceccherini-Nelli ${ }^{I}$

${ }^{I}$ Dipartimento di Patologia Sperimentale BMIE Universita' di Pisa, Pisa, Italy

The evaluation of HIV viral load and drug resistance predicts clinical progression of infection and the effectiveness of antiretroviral 
therapy, but conventional methods of laboratory procedures are feasible only in equipped laboratories. This study evaluates the use of samples of dried whole blood spots (DBS) on filter paper for the analysis of HIV viral load and drug resistance. $200 \mu \mathrm{l}$ of whole blood of 29 patients analyzed in the laboratory of Virology of Pisa by AmpliPrep COBAS ${ }^{\circledR}$ TaqMan HIV-1 Test (Roche) (1 ml of plasma), were placed on FTA ${ }^{\circledR}$ Classic Card (Whatman) and dried. DBS were cut and treated with $1 \mathrm{ml}$ of TE buffer-1 (10 mM Tris- $-\mathrm{HCl}, 0.1 \mathrm{mM}$ EDTA, pH 8.0) over night at room temperature. Supernatants were incubated for $30 \mathrm{~min}$ at $56^{\circ} \mathrm{C}$, and HIV-1 viral load was investigated with the same method. 13 DBS on FTA ${ }^{\circledR}$ Classic Card (Whatman) collected from HIV-positive patients in Central African Republic, in the hospital Giovanni Paolo II Pope, NOI PER L'AFRICA E IL MONDO ONLUS, by finger prick, were sent to the laboratory of Virology of Pisa. HIV-1 viral load was evaluated using the developed protocol. HIV-1 drug resistance was evaluated using PCR and sequencing as previously described by Plantier et al., 2005. Resistance mutations were evaluated using the algorithm available from http://hivdb.stanford.edu/. 29/29 (100\%) DBS were HIV positive: HIV-1 viral load of 7/29 (24.1\%) DBS was the same as in plasma, of $16 / 29(55.2 \%)$ DBS within a logarithm lower, of $6 / 29(20.7 \%)$ DBS within one logarithm higher than in plasma. DBS collected in Central African Republic were tested at least three times: 12/13 (92.3\%) were HIV positive; 6/13 (46\%) contained 105 copies $/ \mathrm{ml}$ (range $11,700-91,600)$ and 4/13 (77\%) 104 copies/ml (range 1,920-4,880). In $2 / 13(15 \%)$ viral load was detectable, but out of the linear range of the instrument $(40-10,000,000$ copies/ml). Drug resistance analysis was performed successfully in $13 / 13$ DBS samples. Using DBS, $200 \mu \mathrm{l}$ of whole blood are sufficient for the evaluation of HIV-1 viral load and drug resistance, with sensitivity in some cases only slightly lower than the routine molecular methods that use $1 \mathrm{ml}$ of plasma. DBS would be a practical and economical method for the collection, storage and transportation of blood samples, especially from developing countries with high prevalence of HIV infection where patients could be followed with the high standard protocols used in the developed countries.

\section{PP 08}

WHAT SCREENING FOR OSTEOPOROSIS IN PATIENTS WITH HIV INFECTION: FRAX SCORE VERSUS BONE SERUM MARKERS!

G. Maffongelli*', A.R. Buonomini ${ }^{1}$, L. Sarmati ${ }^{1}$, S. Gini ${ }^{1}$, L.Dori $^{1}$,

A. Ricciardi ${ }^{I}$, M. Mapfumo ${ }^{I}$, E. Gentilotti ${ }^{I}$, P. Sordillo ${ }^{I}$, M. Andreoni $^{I}$

${ }^{I}$ Cattedra di Malattie Infettive, Tor Vergata, Rome, Italy

Background: There are many observations that correlate metabolic bone disease with HIV infection and HAART regimen. The most common bone diseases described in HIV seropositive patients are osteopenia and osteoporosis. Several diagnostic approaches have been recommended to monitor bone conditions during HIV disease and HAART regimen, as serum parameters, radiological assays, urinary exams. The WHO has developed a fracture risk assessment tool (FRAXtm) based on clinical risk factors with or without bone mineral density tests, for the prediction of fracture in men and woman. The EACS in 2009 recommend FRAXtm calculation in all HIV positive individuals at baseline, every 2 years and in addition prior to commencing ARV therapy.

Methods: To investigate metabolic bone disease, we have submitted 35 HIV-seropositive patients to questionnaire to calculate the FRA$\mathrm{Xtm}$, and to blood tests turn to investigate the markers of bone turnover (D vitamin, parathyroid hormone, calcaemia, phosphatemia, osteocalcin, bone alkaline phosphatase). Bone disease was defined by the presence of abnormal values of at least two serum markers. All the patients performed to radiological tests (hip and lumbar spine DXA, dorsal and lumbar spine radiograph) to certify the body mass density and the detection of fractures.
Results: We studied 35 patients, 33 on HAART and 2 naïve, 22 male and 13 female. The mean age was 41 years (range 27-70). Three patients with a FRAX score above 20 (normal value up 20), had normal values of T Score at DXA and of serum markers. Twelve of the remaining 32 patients with normal values of FRAX score, showed a radiological diagnosis of osteoporosis or osteopenia. Of these 12, 7 had serum markers of high bone turnover. The sensitivity of serum markers to identify bone diseases resulted was $58 \%$ with a specificity of $73 \%$ (positive predictive value 53\% and negative predictive value $77 \%$ ).

Conclusions: In subjects with HIV infection the screening based on bone turnover serum markers is more reliable than the FRAXtm to assess the condition of bone metabolism. These results lead us to revaluate strategies to screen bone damage in HIV-positive patients.

\section{PP 09 \\ HIGH PROPORTION OF HIV-1 SUBTYPE F1 TRANSMISSION NETWORKS AMONG ITALIAN HETEROSEXUAL MALES ASSOCIATED WITH INTRODUCTION EVENTS FROM SOUTH AMERICA}

A. Lai ${ }^{*}$, G. Zehender ${ }^{1}$, A. De Luca ${ }^{2}$, V. Micheli ${ }^{3}$, P. Meraviglia ${ }^{4}$, P. Corsi ${ }^{5}$,

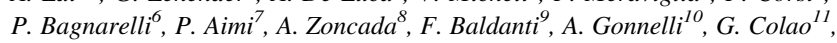
D. Tacconi ${ }^{12}$, F. Simonetti ${ }^{1}$, M. Ciccozzi ${ }^{13}$, M. Zazzi $^{14}$, C. Balotta ${ }^{1}$

${ }^{I}$ Department of Clinical Science, Infectious Diseases and Immunopathology Section, 'L. Sacco' Hospital, University of Milan, Milan, Italy; ${ }^{2}$ Second Infectious Diseases Unit, Siena University, Siena, Italy; ${ }^{3}$ Laboratory of Microbiology, 'L. Sacco' Hospital, Milan, Italy; ${ }^{4} 2$ nd Division of Infectious Diaseases, 'L. Sacco' Hospital, Milan, Italy; ${ }^{5}$ U.O.C. Infectious Diseases

Careggi, Florence, Italy; ${ }^{6}$ Department of Biomedical Science, Section of Microbiology, Laboratory of Virology, University Politecnica delle Marche, Ancona, Italy; ${ }^{7}$ U.O.C. Infectious and Tropical Diseases, Massa; ${ }^{8}$ Infectious Diseases Section, Cremona, Italy; ${ }^{9}$ Section of Virology S. Matteo, Pavia, Italy; ${ }^{10}$ U.O.C. Clinical Infectious Diseases, Siena, Italy; ${ }^{11}$ Section of Virology Careggi, Florence, Italy; ${ }^{12}$ Infectious Diseases Section, Arezzo, Italy;

${ }^{13}$ Epidemiology Unit, Department of Infectious, Parasite and ImmuneMediated Diseases, Italian Institute of Health, Rome, Italy; ${ }^{14}$ Section of Microbiology, Department of Molecular Biology, University of Siena, Siena, Italy

Background: A hallmark of non-B subtype HIV-1 spread in Italy is the very high proportion (about $60 \%$ ) of $\mathrm{F} 1$ variant, circulating at high prevalence in South America and Eastern Europe. Aim of this study was to investigate the features of subtype F1 circulation through phylogenetic approaches.

Methods: We analyzed pol sequences of 343 patients carrying F1 subtype stored in the ARCA database and obtained from 1998 to 2009. All sequences had a subtype assignment through phylogenetic analysis using Phylip package and Simplot software. The transmission networks were identified using the starting tree performed with MrBayes (GTR $+\mathrm{I}+\mathrm{G}$ model). The MCMC search was run for $10 \times 106$ generations sampled every 100th. Only clades with a posterior probability of 1 were considered transmission clusters. Dated phylogeny for each clade were performed using Beast.

Results: Regarding the citizenship, 79.7\% $(\mathrm{n}=208), 8 \%(\mathrm{n}=21)$ and $6.9 \%(\mathrm{n}=18)$ of patients were Italians, South Americans, Rumanians, respectively. The risk factor was as follows: $61.4 \%$ $(\mathrm{n}=126)$ for HEs, $25.4 \%(\mathrm{n}=52)$ for HOs and $13.2 \%(\mathrm{n}=27)$ for IDUs. Males were $255(75.2 \%)$. Place of residence was $46.6 \%$ $(\mathrm{n}=160), 46.9 \%(\mathrm{n}=161)$ and $6.4 \%(\mathrm{n}=22)$ in Northern, Central and Southern Italy, respectively. The phylogenetic analysis indicated that $70 \%(240 / 343)$ of sequences clustered in 27 transmission networks. The 7 larger clusters included 56, 26, 24, 17, 12, 12 and 8 patients. All Rumanian patients were included in the largest cluster, while only $8 / 21(38 \%)$ South Americans belonged to 8 distinct clusters. The heterosexual modality of infection was significantly associated with the probability to be detected in transmission networks $(78.6 \%$ HEs vs. $63.5 \%$ HOs, $\mathrm{p}=.03)$. HEs were prevalent either among Italians $(77.9 \%, \mathrm{n}=81)$ or Romanians $(100 \%, \mathrm{n}=6)$; 
by contrast, HOs accounted for $71.4 \%$ of South American patients $(\mathrm{p}=.007)$. A significantly higher proportion of clustering sequences belonged to patients residing in the Northern part of Italy $(52.1 \%$ for North, $40.4 \%$ for Center and $7.5 \%$ for South, $\mathrm{p}=.001$ ). Within the epidemiological networks the Italian HEs males $(74.3 \%, \mathrm{n}=65)$ were predominant, while males and females among HEs Rumanians were equally distributed ( $40 \%$ males and $60 \%$ females). Dated phylogeny, based on Time of Most Recent Common Ancestor, evaluated in conjunction with citizenship of patients, indicated that the majority of older clusters included South American HOs.

Conclusions: A very high proportion of epidemiological clusters could be identified in heterosexual individuals carrying F1 subtype. Their proportions are equally distributed in the North and in the Centre of Italy, however transmission networks were prevalent at North. Foreign patients were mainly Rumanian males or females and South American HOs. Italian HE males predominated within transmission clusters. Dated analysis suggests that F1 variant from South America gave rise to the Italian F1 epidemic through multiple introduction events.

\section{PP 10}

\section{HIV INCIDENCE AND CHARACTERISTICS OF NEWLY} DIAGNOSED HIV INFECTIONS AMONG NON-NATIONALS IN ITALY, 2003-2009

L. Camoni ${ }^{\prime}{ }^{l}$, V. Regine ${ }^{I}$, M.C. Salfa ${ }^{l}$, M. Raimondo ${ }^{l}$, B. Suligoi ${ }^{l}$ e i Referenti regionali del Sistema di sorveglianza delle nuove diagnosi di infezione da HIV ${ }^{2}$ ${ }^{1}$ Istituto Superiore di Sanità, Rome, Italy; ${ }^{2}$ A. Carboni (Lazio); R. Cassiani (Abruzzo); M. Chironna (Puglia); M. Cuccia (Catania); A.C. Finarelli (EmiliaRomagna); G. Greco (Calabria); G. Icardi (Liguria); M.S. Mura (Sassari); C. Pasqualini (Piemonte); A. Pavan (Lombardia); C. Piovesan (Veneto); G. Rossetti (Trento); R. Pristerà (Bolzano); N. Scola (Marche); L. Sudano (Valle d'Aosta); C. Zappetti (Friuli-Venezia Giulia)

Introduction: In the Italian regions where a surveillance system for new HIV diagnoses is operating, the proportion of non-nationals among new HIV diagnoses has increased, from $11.0 \%$ in 1992 to $29.7 \%$ in 2003, and leveled-off thereafter. The objective of the present study is to analyze the characteristics of non-nationals with a new HIV diagnosis and the incidence of HIV infection among nonnationals between 2003 and 2009.

Methods: Data on HIV diagnoses were provided by those regions where a surveillance system is operating. In 2009, the residents in these regions accounted for $70.6 \%$ of the total resident population. We defined as "non nationals" those persons born outside of Italy, or with foreign nationality, or foreign citizenship. The incidence among non-nationals was estimated as the annual number of new HIV diagnoses among non-nationals (numerator) divided by the annual number of non-nationals legally residing in the same areas (denominator) per 100,000 . Data on the number of legally resident nonnationals were obtained from the National Institute of Statistics (ISTAT) and are available since 2003.

Results: Between 2003 and 2009, 3,828 new HIV diagnoses were reported among non-nationals, accounting for $29.7 \%$ of the 12,875 new HIV diagnoses reported by the regional surveillance systems. The distribution by geographic area showed that $52.6 \%$ of nonnationals originated from Africa, 28.2\% from South America, 7.0\% from Eastern Europe, $5.1 \%$ from Asia, and the remaining originated from other European countries, Central-North America, and Oceania. Males accounted for $52.0 \%$ of the total. The highest proportion of cases was reported in the age group 30-34 years (22.8\%). Heterosexual contact was the most common exposure category, being reported by $51.3 \%$ males and $79.4 \%$ females. Homosexual contact was reported by $23.0 \%$ males. The number of legally resident nonnationals was 568,610 in 2003 and 3,169,843 in 2009. The incidence of HIV infection among non-nationals significantly decreased ( $p<0.0001$ ), from 77.6 new diagnoses per 100,000 residence permits in 2003, to 22.6 new diagnoses per 100,000 residence permits in 2009 . In the same period, incidence among Italians significantly decreased ( $p<0.0001$ ), from 6.4 new diagnoses per 100,000 residents in 2003, to 4.9 new diagnoses per 100,000 in 2009 .

Conclusions: After 2003, despite of the increasing number of legally resident non-nationals in Italy, the incidence of HIV infection among non-nationals has markedly decreased. This result suggests that targeted prevention campaigns and facilitated access to testing and clinical management have been successfully implemented among non-nationals in the last decade. However, in 2009, incidence of new HIV infections among non-nationals was five-fold higher than that observed among Italians, stressing the need to reinforce the efforts aimed at reducing the circulation of HIV in this population.

\section{PP 11 NEWLY DIAGNOSED OF HIV INFECTION AMONG YOUNG MEN WHO HAVE SEX WITH MEN

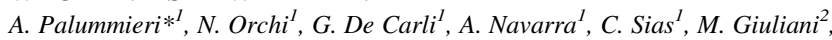 P. Scognamiglio ${ }^{I}$, S. Pittalis ${ }^{I}$, S. Grisetti ${ }^{\text {I }}$, A. Corpolongo ${ }^{I}$, A. Sampaolesi ${ }^{l}$, V. Puro ${ }^{I}$, E. Girardi for the SENDIH group ${ }^{l}$ \\ ${ }^{I}$ National Institute for Infectious Diseases "L. Spallanzani", Rome, Italy; \\ ${ }^{2}$ AIDS Center "San Gallicano" Institute, Rome, Italy}

Background: Rising rates of HIV infections among young adults ( $<30$ years), mainly men having sex with men (MSM) has been observed, suggesting an increase in high-risk behaviours in this population. The aim of this study is to highlight epidemiological and behavioural characteristics of young MSM newly diagnosed with HIV infection in Lazio Region, Italy.

Methods: Since January 2004, a cross sectional multi-centre study, involving 14 public counselling and testing sites, has been enrolling all adult patients newly diagnosed with HIV. At diagnosis, demographic, epidemiological, clinical and laboratory data are collected, and patients are asked to complete a questionnaire investigating socio-psycho-behavioural aspects. Individuals are identified as recent infection (RI) combining conventional laboratory methods for seroconversion with an avidity assay as antibody biomarker for RI. Among long-standing infections, we defined as having a late diagnosis individuals with a CD4 cells count $<350 / \mathrm{mmc}$ or clinically diagnosed with AIDS.

Results: Between 2004 and 2009, 2,225 newly diagnosed HIV infections were observed. Of $501(23 \%)$ young adults, $282(56.3 \%)$ were MSM, of whom 92 aged 18-25 years. No temporal trend of reported cases among MSM was observed. More than one-third (95) of diagnoses involved foreign born individuals, $75 \%$ of whom originated from Latin America. For 97 (34.4\%) of MSM, HIV infection was diagnosed at their first HIV test. Of $168(59.6 \%)$ previously tested, $75(26.5 \%)$ reported to be tested in the 12 months before the HIV diagnosis. The median CD4 cell count at diagnosis was 490 cells/mmc (range 4-1,680) and the median HIV viral load was 36,689 copies/ml (range 49-1,900,000); 4\% had an AIDS defining illness. $46(16.3 \%)$ individuals received a diagnosis of syphilis at the same time (20) or in the months preceding HIV diagnosis. With regard the stage of infection, for 57 individuals (with a CD4 cell count $>350$ cells/mmc or missing, or without avidity test) was not possible to define the stage of infection. 180/225 MSM (80.0\%) were longstanding infections. Of these, 68 had a late diagnosis. Of the $45 \mathrm{RI}$ identified, $23(51.2 \%)$ were classified through the avidity assay. Of the 282 young MSM diagnosed, 114 (40\%) accepted to answer to the behavioral questionnaire. Among these, 104 (91\%) reported having $>1$ casual male sex partner during the 12 months prior the HIV diagnosis; 63/104 reported $>5$ partners. Of 114 responders, 40 and $96 \%$, respectively, reported unprotected anal or oral sex, and $50 \%$ reported using illegal drugs before o during sex.

Discussion: In spite of a high prevalence of reported risk behaviours, only a minority of young MSM are diagnosed early after HIV 
infection. Further efforts are needed to increase the demand for and availability of HIV testing to help young MSM at risk for HIV receive prevention counselling, learn their status, and receive referrals for HIV treatment.

\section{PP 12 \\ EXPANDED POSTEXPOSURE PROPHYLAXIS: THE IRAPEP EXPERIENCE

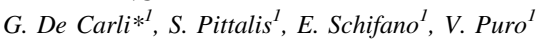 \\ Istituto Nazionale Malattie Infettive L. Spallanzani, Rome, Italy}

Current guidelines recommend a standard triple ARV regimen, generally 2 NRTI + PI, as post-exposure prophylaxis (PEP) after HIV atrisk exposures; an 'expanded' regimen could be considered in case of exposures to sources infected with multi-resistant strains, despite concerns on enhanced risk of toxicity and hence lower adherence. ARV drugs of more recent availability and for which tolerability in HIV negative subjects has not been assessed are used with extreme caution to balance between potential risks and possible benefits. In particular, scarce data are available on entry and integrase inhibitors, those offering a theoretical advantage as PEP. To assess tolerability and outcome of 'expanded' PEP regimens, we reviewed the Italian Registry of Antiretroviral PEP searching for all the treatments with $>3$ drugs, administered from 2004, when enfuvirtide became available, to 2010. 1,850 PEP treatments were reported, 51\% occupational (799 healthcare workers), and $49 \%$ nonoccupational (710 sexual). Eight cases treated with an 'expanded' PEP regimen were identified $(0.4 \%)$ : five following occupational exposures (4 injuries with hollow-bore, blood filled needles and 1 eye splash with blood) plus one in a patient's relative performing a subcutaneous injection; and two after sexual exposures (vaginal insertive and anal receptive contact). All exposures involved ARV-experienced sources infected with multi-resistant strains, except one involving a patient with acute primary HIV infection who later resulted infected with a multi-resistant strain. Two sources had undetectable viremia at the time of exposure, while six had viral load ranging from 48 to $170,000,000$ copies $/ \mathrm{mL}$. Six were coinfected with hepatitis C. Exposed subjects received 2 NRTI (6 $\mathrm{ZDV}+3 \mathrm{TC}, 2 \mathrm{TDF}+\mathrm{FTC})+1$ boosted PI (4 LPV, $1 \mathrm{TPV}, 3$ $\mathrm{DAR})+$ an entry inhibitor (5 T20, $2 \mathrm{MVC})$ and/or an integrase inhibitor (3 RAL). Treatment was generally well tolerated, and all but two completed the 4-week PEP course. Two subjects interrupted PEP because of adverse effects, one at day 24 because diarrhea was accompanied by hemorrhoidal bleeding; the other at day 9 because of severe nausea and abdominal pain due to meteorism. T20 administration determined mild injection site reactions in all subjects. All subjects were HIV and HCV negative at follow-up testing after 6 months. The recourse to 'expanded' PEP is infrequent, based on the severity of exposure more than on the infectivity of the source, on the basis of documented resistance in the patient history and not necessarily on current evidence of virological failure. Broader use of new class drugs and further safety data may provide future rationale to expand its use, under close clinical and biological monitoring.

IRAPEP members contributing to this study: Giuseppe De Socio, Perugia; Luca Fabbri, Pistoia; Eugenio Mantia, Alessandria; Paola Scognamiglio, Roma; Marcello Tavio, Udine; Valerio Tozzi, Roma.

\section{PP 13}

\section{EFFICACY, SAFETY AND TOLERABILITY OF CO-FORMULATED EFV/FTC/TDF TREATMENT.}

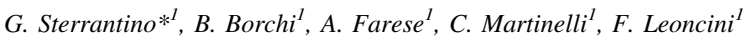

${ }^{I}$ Azienda Ospedaliera-Universitaria Firenze, Florence, Italy

Background/objective: Co-formulated EFV/FTC/TDF is the first once daily single tablet antiretroviral (ARV) regimen approved in the United States, Canada, and the EU. The components have demonstrated long-term efficacy and safety in treatment naive patients. The objective of this study was to evaluate in clinical practice whether patients who simplified their current ARV regimen to a single tablet regimen of EFV/FTC/TDF had similar effectiveness (efficacy, safety and tolerability).

Methods: We performed retrospective assessment of HIV+ patients who switched from PI containing regimens to co-formulated EFV/ FTC/TDF at our institution. $155 \mathrm{HIV}-1+$ patients have been enrolled: all patients included in the study had HIV-RNA less than $50 \mathrm{UI} / \mathrm{mL}$ at switch, median age was 47 years (IQR 40-53), male 84\%, IDUs 17\%, MSM $42 \%$, heterosexuals $30 \%$, unknown $10 \%$, transfused $1 \%$; $38 / 145$ patients $(26 \%)$ were $\mathrm{HCV}$ or HBV co-infected, 10 unknown. HIV-subtype, assessed in 101 patients, was B in $87 \%$, non-B in $13 \%$. Median CD4 nadir was 259 cells/mL (IQR 171-349). Thirty-one patients $(20 \%)$ had AIDS history. Median time of HIV infection was 9 years (IQR 6-16). Forty-seven out of 99 patients had on historical genotype at least one major mutations for NRTI (35\%), NNRTI (19\%), PI (13\%). Median CD4 cell count at switch was 648 cells $/ \mathrm{mL}$ (IQR 493-835). The association of FTC, TDF and EFV was first-line therapy (Group 1) in 45 patients (29\%), 61 patients (39\%) had previously assumed 1 PI (Group 2), 49 patients (32\%), at switch, were multi-experienced (=2 PI) (Group 3). After switch to co-formulated EFV/FTC/TDF median follow-up was 25 months (IQR 20-28). CD4, HIV-RNA, total cholesterol, triglycerides, creatinine, AST and ALT were evaluated at switch and every $3 / 4$ months.

Results and conclusions: 137 out of 155 (88.4\%) patients had undetectable viremia at the end of follow-up. CD4 median monthly increase was 3 cells/ml (IQR $-1 /+7)$. Eighteen patients (12\%) discontinued co-formulated EFV/FTC/TDF, four due to virological failure: two out of four developed NNRTI resistance (the first one $103 \mathrm{~N}$ and 190A mutations) and the second one (101E and $103 \mathrm{~N}$ mutations), one had $103 \mathrm{~N}$ mutations on historical genotype, one failed without developing of resistance. Five patients stopped coformulated EFV/FTC/TDF treatment due to neurological efavirenz side effects, seven patients developed proteinuria and/or creatinine increase, one severe hepatic steatosis, one CPK increase. Eleven out of 18 patients $(61 \%)$ who discontinued study treatment belonged to Group 3, their median age was 50 years (IQR 43-61), and 33\% had AIDS diagnosis. We did not found statistically significant differences among values of transaminases, total cholesterol, triglycerides before and after co-formulated EFV/FTC/TDF switch.

\section{PP 14}

COMPARISON OF HIV-1 RECOMBINANT PHENOTYPIC ASSAYS AND VIRTUAL PHENOTYPE IN THE DEFINITION OF CORECEPTOR USAGE

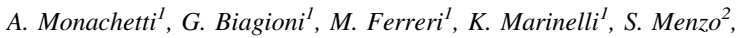

P. Bagnarelli* ${ }^{I}$

${ }^{1}$ Virology Unit, Department of Biomedical Sciences, University Politecnica Marche, Ancona, Italy; '2aboratory of Virology, INMI "L. Spallanzani", Rome, Italy

Aims: The introduction in clinical practice of CCR5 antagonists as a new class of entry inhibitors has determined the need to know the coreceptor usage before starting therapy. Most clinicians obtain this information through the Trofile assay, that in the last version is characterized by enhanced sensitivity (ESTA). The widespread use of this assay is impractical for the high cost and the long turnaround time. Several attempts have been made to achieve faster, cheaper and reliable results by improving genotypic interpretation algorithms. However, because of the high variability of the env region, genotypic assays can still be unreliable, while phenotypic biological assays are almost always capable of defining the actual tropism. A simple and sensitive recombinant phenotypic assay would still prove useful: the goal of the paper was to compare an in-house HIV recombinant phenotypic assay to ESTA and both phenotypic assays to the performances of a genotypic HIV-1 tropism prediction algorithm. 
Methods: Recombinant DNA techniques were used to introduce a blunt-end cloning cassette in the replicating HIV-NL4-3 molecular clone in order to clone different V3 sequences from patient virus in the replicative backbone for phenotypic testing. A GFP reporter (engineered as a nef fusion product in the backbone) allowed viral replication to be determined by fluorimetric analysis of CCR5 or CXCR4 expressing cell lines following transfection. Virtual phenotype was obtained by V3-loop RNA genotype-based geno2pheno (G2P) interpretation setting false positive rate (FPR) at $10 \%$.

Results: 52 recombinant viruses were obtained from plasma samples of 21 HIV-1 patients. Coreceptor usage was analyzed in all recombinant clones indicating R5 tropism in 33 and DM/X4 tropism in 11 clones corresponding to 12 and 9 patients, respectively, a not reportable (NR) phenotype was observed in 8 viral chimerae obtained from 5 of the 21 patients. ESTA was determined in 13 out of 21 patients indicating R5 in 8, DM/X4 in 4 and NR in 1, that resulted $\mathrm{DM} / \mathrm{X} 4$ by our recombinant assay. On the whole, there was perfect correspondence between the two biological assays, and between G2P prediction and ESTA. Some discrepancy was observed comparing G2P prediction of the molecular clones and their coreceptor usage. The viral chimerae from 6 out of $14 \mathrm{~V} 3$ loop evaluated as probably using CXCR4 by a G2P FPR $<10 \%$ showed, in fact, an R5 phenotype. Interestingly, the sequence's FPR was $>5 \%$ in five of those six.

Conclusions: The recombinant phenotypic assay described is highly comparable to ESTA moreover it offers the possibility to dissect the composite phenotype of viral quasispecies at the clonal level providing information about the influence of specific aminoacidic residue. Interestingly, $43 \%$ of $\mathrm{V} 3$ clonal sequences, predicted as probable $\mathrm{X} 4$ by virtual algorithm, conferred an R5 tropism when inserted into NL4-3. The threshold level of FPR should be considered with great attention.

\section{PP 15}

\section{FACTORS IMPACTING HUMAN IMMUNODEFICIENCY VIRUS-} TYPE 1 CORECEPTOR TROPISM

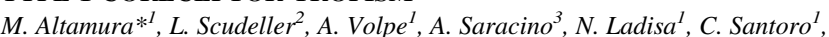
L. Monno ${ }^{\text {, G. Angarano }}{ }^{1}$

${ }^{I}$ Clinica Malattie Infettive, Università degli Studi di Bari, Bari, Italy; ${ }^{2}$ IRCCS Policlinico San Matteo, Pavia; ${ }^{3}$ Clinica Malattie Infettive, Università degli Studi di Foggia, Foggia, Italy

Background: Determination of HIV-1 coreceptor tropism (CTR) is necessary before prescribing the CCR5 antagonist maraviroc (MVC). Consequently, studies regarding coreceptor usage in different populations are required to identify the most suitable candidates for use of MVC and its optimal allocation in antiretroviral regimens. Herein, CTR in chronic HIV-1 infected patients was evaluated together with its possible association with risk factors for the acquisition and duration of infection and the clinical and virological parameters involved.

Methods: HIV-1 CTR (enhanced sensitivity trofile assay, ESTA) was evaluated in 208 drug-naïve/experienced patients. ESTA was successful in $187(90 \%)$ patients, $129(68.9 \%)$ and $58(31 \%)$ of whom harboured a R5 and X4 virus (either pure or dual/mixed), respectively. The association between CTR and selected variables was analyzed by means of $t$ test, Mann-Whitney, or chi-square test, as appropriate. Univariate and multivariate logistic regression was used to assess predictors of CTR; interaction between nadir CD4+ count $<200$ cells/mmc and antiretroviral therapy (naïve, current therapy, interrupted therapy) as well as confounding and colinearity between variables were also tested.

Results: Patients were mostly males $(73 \%)$ of Italian origin $(88 \%)$ with a mean age of 39.7 years, and according to the self-reported risk factors, included 131 (70\%) sexually infected subjects (either hetero, homo or bisexuals) and 37 (19.7\%) intravenous drug users (IDUs); 46 $(24.5 \%)$ patients were infected with a non subtype B virus. At the time of testing, $53(28.3 \%)$ had a AIDS diagnosis, and $99(53 \%)$ had nadir $\mathrm{CD} 4+<200$. The median $\mathrm{CD} 4+$ and plasma viral load were 297 cells/ $\mu$ l and $4.51 \log _{10}$ HIV-RNA copies/ml, respectively. At univariate analysis, variables associated with CTR at the 0.1 level were the following: years of known HIV duration, CD4 $<200$ cells/ mmc, nadir CD4, era of HIV infection ( $<1997$ vs. $\geq 1997)$, intravenous drug abuse, clade, therapy experience, AIDS diagnosis. At multivariate analysis, the only independent variable associated with CTR was nadir CD4 $<200$ cells $/ \mathrm{ml}$ with a 3.8 times increased risk (95\% CI $1.76-8.58, p=0.001)$ of having an $\mathrm{X} 4$ variant.

Conclusion: Similar to recent studies, the only variable independently associated with CTR was a nadir CD4 count of $<200 / \mathrm{ml}$. The close relationship between X4 and nadir CD4 further emphasizes the necessity for early HIV testing and HIV diagnoses. Moreover, it also suggests that the optimal allocation for maraviroc should be as early as possible within the initial regimens, an advice currently acceptable according to recent guidelines.

PP 16

THERAPEUTIC DRUG MONITORING OF ANTIRETROVIRAL DRUGS IN HIV-INFECTED PREGNANT WOMEN:

PHARMACOKINETICS, TRANSPLACENTAL AND AMNIOTIC FLUID DIFFUSION, EFFICACY AND SAFETY

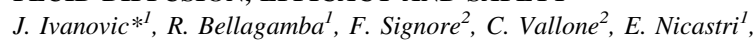
M. Tempestilli ${ }^{1}$, G. Pisani ${ }^{2}$, C. Tommasi ${ }^{1}$, A.L. Gallo ${ }^{I}$, P.L. Pucillo ${ }^{l}$, P. Narciso ${ }^{1}$

${ }^{I}$ National Institute for Infectious Diseases 'Lazzaro Spallanzani', Rome, Italy; ${ }^{2}$ Department of Obstetrics and Gynaecology, 'San Camillo-Forlanini'

Hospital, Rome, Italy

Background: The aim was to evaluate antiretroviral (ARV) drugs levels during three trimesters of pregnancy, transplacental and amniotic fluid diffusion and clinical maternal and newborn outcome. Methods: Antiretroviral concentrations were determined in mother and cord blood and in amniotic fluid by high-performance liquid chromatography. Cord-to-mother ratio (C:M) was calculated to estimate the ARVs placental passage. HIV viral load and CD4 cell count values were recorded in first, second, third trimester and at delivery. Newborn gestational age, weight, Apgar score and complications were recorded at birth.

Results: Seventy-three HIV-infected pregnant women were enrolled. Maternal nevirapine (NVP, $\mathrm{n}=14$ ) mean Ctrough was 3,629 \pm $956 \mathrm{ng} / \mathrm{mL}$ with a mean $\mathrm{C}: \mathrm{M}$ ratio of $0.68 \pm 0.1$ and amniotic fluid level of $1,257 \mathrm{ng} / \mathrm{mL}$ obtained from one sample; maternal nelfinavir $(\mathrm{NFV}, \mathrm{n}=7)$ Ctrough was $837 \pm 673 \mathrm{ng} / \mathrm{mL}$ with a mean C:M ratio of $0.58 \pm 0.18$; maternal atazanavir (ATV, $\mathrm{n}=8)$ Ctrough was $1,309 \pm 609 \mathrm{ng} / \mathrm{mL}$ with a mean $\mathrm{C}: \mathrm{M}$ ratio of $0.20 \pm 0.17$ and mean amniotic fluid level of $286 \pm 47 \mathrm{ng} / \mathrm{mL}$; maternal saquinavir (SQV, $\mathrm{n}=2$ ) Ctrough was $903 \pm 137 \mathrm{ng} / \mathrm{mL}$ with a mean C:M ratio of $0.13 \pm 0.03$; maternal fosamprenavir $(\mathrm{FPV}, \mathrm{n}=6)$ Ctrough was $696 \pm 401 \mathrm{ng} / \mathrm{mL}$ with a mean C:M ratio of $0.18 \pm 0.09$ and mean amniotic fluid level of $468 \pm 58 \mathrm{ng} / \mathrm{mL}$; maternal lopinavir (LPV, $\mathrm{n}=31$ ) Ctrough was $4,113 \pm 2,465 \mathrm{ng} / \mathrm{mL}$ with C:M ratio $0.1 \pm 0.2$ and mean amniotic fluid level of $198 \pm 125 \mathrm{ng} / \mathrm{mL}$; maternal darunavir (DRV, $\mathrm{n}=5$ ) Ctrough was 2,014 $\pm 1,549 \mathrm{ng} / \mathrm{mL}$ with a mean $\mathrm{C}: \mathrm{M}$ ratio of $0.12 \pm 0.04$ and mean amniotic fluid level of $403 \pm 437 \mathrm{ng} / \mathrm{mL}$. ATV, DRV and FPV were more concentrated in amniotic fluid than in cord blood. The observed prevalence rate of neonatal low birth weight (LBW, $<2,500 \mathrm{~g}$ ) and preterm delivery (PTD, $<37$ week) was $22 \%(\mathrm{n}=16)$ and $17.8 \%(\mathrm{n}=13)$, respectively. LBW and PTD was observed in $14.3 \%(\mathrm{n}=2)$ newborns of mothers treated with NVP.; in $25.8 \%(\mathrm{n}=8)$ and $42.9 \%(\mathrm{n}=3)$, respectively of newborns exposed to LPV; in $28.6 \%(\mathrm{n}=2)$ and $42.9 \%(\mathrm{n}=3)$, respectively of newborns exposed to NFV, in $37.5 \%$ $(\mathrm{n}=3)$ and $12.5 \%(\mathrm{n}=1))$, respectively of newborns exposed to ATV, in $20 \%(n=1)$ of those exposed to DRV. There were no cases of LBW and PTD for FPV and SQV. All women treated during 
pregnancy only for prevention of HIV vertical transmission maintained an immunological set-point at follow-up above the nadir CD4 cell count. Ctrough below recommended drug Cmin was not significantly associated to virological failure during follow-up $(\mathrm{p}=0.01)$. Conclusions: Measurement of antiretroviral exposure in different compartments during pregnancy may be needed to identify sub- or supra-therapeutic drug exposure. Further, larger study population PK, safety and maternal viro-immunological data are warranted to assist in selecting optimal drug regimens and to justify implementation of antiretroviral dose adjustment during pregnancy.

\section{PP 17}

INCREASED USE OF NEW GENERATION ANTIRETROVIRAL DRUGS IN ITALIAN CHILDREN WITH PERINATAL HIV INFECTION

E. Chiappini*', L. Galli ${ }^{1}$, P. Tovo ${ }^{2}$, C. Gabiano ${ }^{2}$, C. Lisi $^{3}$, A. Vigano $^{4}$,

S. Bernardi ${ }^{5}$, S. Esposito ${ }^{6}$, R. Rosso ${ }^{7}$, C. Biaquinto ${ }^{8}$, R. Badolato ${ }^{9}$,

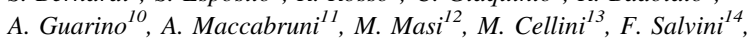

D. La Rovere ${ }^{15}$, M. Dedoni ${ }^{16}$, I. Dodi ${ }^{17}$, M. de Martino

${ }^{I}$ Department of Science for Woman and Child Health, University of Florence,

Florence, Italy; ${ }^{2}$ Department of Pediatrics, University of Turin, Turin, Italy;

${ }^{3}$ Department of Statistics, University of Florence, Florence, Italy; ${ }^{4}$ Department of Pediatrics, University of Milan, L. Sacco Hospital, Milan, Italy; ${ }^{5}$ Pediatric Clinic, "Bambino Gesù" Hospital, Rome, Italy; ${ }^{6}$ Department of Maternal and Pediatric Sciences, Milan University, Fondazione IRCCS Ospedale Maggiore Policlinico, Mangiagali e Regina Elena, Milan, Italy; ${ }^{7}$ Infectious Diseases Clinic, University of Genoa, San Martino Hospital, Genoa, Italy; ${ }^{8}$ Department of Pediatrics, Padua University, Padua, Italy; ${ }^{9}$ Pediatric Clinic, Brescia University, Brescia, Italy; ${ }^{10}$ Department of Pediatrics, "Federico II" University, Naples, Italy; ${ }^{11}$ Department of Infectious Diseases, University of Pavia, Pavia, Italy; ${ }^{12}$ Pediatric Clinic, "S. Orsola" Hospital, Bologna University, Bologna, Italy; ${ }^{13}$ Department of Mother and Child, University of Modena, Modena, Italy; ${ }^{14}$ Division of Pediatrics, University of Milan, S. Paolo Hospital, Milan, Italy; ${ }^{15}$ Pediatric Infectious Disease Unit. Giovanni XXII Hospital, Bari, Italy; ${ }^{16}$ Pediatric Clinic, Cagliari University, Cagliari, Italy; ${ }^{17}$ Pediatric Department, University Hospital of Parma, Parma, Italy

Background: Information regarding the use of new antiretroviral drugs in children in the real setting of clinical fields is largely unknown.

Objective: To provide information regarding modifications to the use of antiretroviral drugs since 1996 and to explore factors possibly associated with virological and immunological outcomes in a large cohort of Italian children with perinatal HIV-infection

Methods: Data from 2,554 combined antiretroviral therapy (cART) protocols administered between 1996 and 2009 to 911 children enrolled in the Italian Register for HIV infection in children, were analyzed. Factors potentially associated with undetectable viral load and immunological response to cART were explored by Cox regression analysis. Results: The proportion of protease inhibitor-(PI) based regimens significantly decreased from 88.0 to $51.2 \%$ and $54.9 \%$, while the proportion on non-nucleoside reverse transcriptase inhibitor (NNRTI)-based regimens increased from 4.5 to $38.8 \%$, and $40.2 \%$ in 1996-1999, 2000-2004 and 2005-2009, respectively ( $\mathrm{P}<0.0001)$. Significant change in the use of each antiretroviral drug occurred over the time periods $(\mathrm{P}<0.0001$ for every drug). Factors independently associated with virological and immunological success were: calendar period, age at protocol, and baseline CD4+ T-lymphocyte percentage. Use of unboosted PI was associated with lower adjusted Hazard Ratio (aHR) of virological or immunological success with respect to NNRTI- and boosted PI-based protocols, with no difference among these two latter types. Among NNRTIs, efavirenz use was more likely associated with virological success than nevirapine (aHR: 4.306; 95\% CI 1.745-10.626; P = 0.002). Conclusion: Use of new generation antiretroviral drugs in Italian HIV-infected children is increasing. "Old" drugs, such as unboosted PIs, were associated with worse outcomes, while no difference was observed between NNRTI- and boosted PI-based protocols. Age and baseline CD4+ $\mathrm{T}$ percentage were independent predictors of immunological and virological success, confirming that the "heat early" strategy remains fundamental.

\section{PP 18}

NO CORRELATION BETWEEN RISK OF NEUROPSYCHIATRIC ADVERSE EVENTS AND CSF PENETRATION EFFECTIVENESS (CPE) SCORE IN THE MONET TRIAL OF DARUNAVIR/RITONAVIR (DRV/R), WITH OR WITHOUT NUCLEOSIDE ANALOGUES (NRTIS)

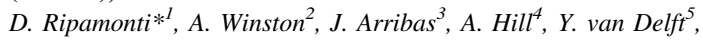
C. Moecklinghoff ${ }^{6}$

${ }^{1}$ Ospedali Riuniti di Bergamo, Italy, Bergamo; ${ }^{2}$ St Mary's Hospital, London, UK; ${ }^{3}$ Hospital la Paz, Madrid, Spain; ${ }^{4}$ Pharmacology Research Laboratories, University of Liverpool, Liverpool, UK, ; ${ }^{5}$ Janssen-Cilag EMEA, Tilburg, The Netherlands; ${ }^{6}$ Janssen-Cilag EMEA, Neuss, Germany

Background: A higher "CSF Penetration Effectiveness Score" (or $\mathrm{CPE}$ score) has been correlated with a lower risk of neurological disorders in cohort studies, but the CPE score has not been validated in randomized clinical trials.

Methods: In the MONET trial, 256 patients with no history of virological failure and HIV RNA $<50$ copies/mL on current HAART for over 24 weeks, switched to DRV/r 800/100 mg once daily, either as monotherapy or with 2NRTIs. The number of patients with clinical reported neuropsychiatric Grade 1-4 adverse events up to Week 96 was analysed by treatment arm, and correlated with the median CPE score. Patients also prospectively completed the FAHI questionnaire which included questions on cognitive function (memory, attention and concentration).

Results: Patients were $81 \%$ male and $91 \%$ Caucasian, with median age 43 years, and median CD 4 count of 575 cells $/ \mu \mathrm{L}$. The median CPE score during the trial was 3 in the DRV/r arm versus 8 in the DRV/r + 2NRTI arm. By Week 96, Grade 1-4 neuropsychiatric adverse events were reported in $29.9 \%$ of patients for DRV/r versus $27.1 \%$ in the DRV/r +2 NRTI arm. The total number of Grade $1-4$ neuropsychiatric adverse events reported was 52 in the DRV/r arm versus 63 in the DRV/r + 2NRTI arm. When the adverse events were weighted by severity (i.e. Grade 1 event: 1 point, Grade 2 event: 2 points, Grade 3 event: 3 points), the total score for neuropsychiatric adverse events was 77 in the DRV/r arm versus 88 in the DRV/ $\mathrm{r}+2 \mathrm{NRTI}$ arm. In the FAHI questionnaire, the mean score of Cognitive Functioning did not change significantly between baseline and Week 96 in either treatment group.

Conclusions: In the MONET trial, the median CPE score was 3 in the $\mathrm{DRV} / \mathrm{r}$ arm versus 8 in the DRV/r + 2NRTIs arm; however there was no difference in the frequency or severity of neuropsychiatric adverse events between the treatment arms, when measured by several different methods.

\section{PP 19}

MARAVIROC (MVC) REDUCES LIVER STIFFNESS PROGRESSION (LSP) IN HIV-HEPATITIS C (HCV) COINFECTED PATIENTS. PRELIMINARY DATA FROM A RANDOMIZED CONTROL STUDY (MAICOL)

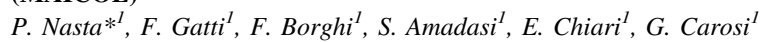

${ }^{I}$ Istituto di Malattie Infettive e Tropicali, Brescia, Italy

Background: in HIV/HCV patients (pts) LSP is faster than in HCVmonoinfected. The CC chemokines MIP-1alpha, MIP-1beta and RANTES and their receptors CCR1 and CCR5 are strongly upregulated in experimental mouse models of fibrogenesis. We investigated the influence of CCR5 inhibition due to MVC on LSP in HIV/HCV coinfected persons.

Methods: HIV/HCV pts, anti HCV treatment naïve, on stable effective HAART with atazanavir/ritonavir $300 / 100 \mathrm{mg}+$ tenofovir/ emtricitabine QD and Child-Pugh score $<$ A6 were enrolled in a 
96 weeks (W), prospective, randomized, pilot study. LSP has been evaluated with biochemical markers of liver fibrosis and transient elastometry, performed by standard methods. Eligible subjects have been randomized 1:1 to maintain the current regimen ( $\operatorname{arm} \mathrm{A}$ ) or to add MVC $150 \mathrm{mg}$ BID (arm B). Clinical, virologic, immunologic, hepatic and metabolic parameters are recorded at baseline (BL) and every 3 months. Liver stiffness (LS) was measured every $24 \mathrm{~W}$ and staged following the standardized categories I: $<7.1 \mathrm{kPa}$; II: 7.1-9.4; III: 9.5-12.4; IV: $\geq 12.5$. A preliminary $24 \mathrm{~W}$ analysis has been assessed. The Mann-Whitney/Wilcoxon test has been used to compare the median of LS variations from BL through $24 \mathrm{~W}$ in two groups.

Results: Up to September 2010, 54 pts were enrolled: 28 in arm B, $77 \%$ males, median age 46 (IQR 43-48) years, CD4 506 (405-654) cells $/ \mathrm{mm}^{3}$, AST 43 (31-59) IU/ml, ALT 68 (45-92) IU/ $\mathrm{ml}$, HCV $5.8(5.4-6,2) \log _{10} \mathrm{IU} / \mathrm{ml}$. LS was $7.2 \mathrm{kPa}(4.2-10.2)$ in arm $\mathrm{B}$ and $5.7 \mathrm{kPa}(4.7-7.4)$ in $\operatorname{arm} \mathrm{A}(\mathrm{p}=\mathrm{NS})$. Twenty-four subjects achieved week 24 (12 patients in each arm). From BL to week 24 LS increased $[+0.35 \mathrm{kPa}(-0.2 ;+1.4)]$ in subjects enrolled arm $\mathrm{A}$ and decreased $[-0.5(-1.7 ;+0.3)]$ in subjects enrolled in arm $B(p=0.03)$. Seven of 24 patients (3 in arm A and 4 in arm B) (29.1\%) changed the stage of LS: In all $3(100 \%)$ patients enrolled in arm A, LS worsened, switching from stage I to stage II. Three out of four pts enrolled in arm B had a LS improvement switching from stage III to stage II.

Conclusion: To add MVC to the current HAART in HIV/HCV coinfected subjects is safe and well tolerated. Moreover in pts randomised to receive MVC a trend toward a reduction in LS has been detected.

\section{PP 20}

SIX-YEAR CHANGES OF ANTHROPOMETRIC, TOTAL AND APPENDICULAR FAT MASSES MEASURES IN HIV-INFECTED CHILDREN SWITCHING FROM STAVUDINE TO TENOFOVIR AND FROM PROTEASE INHIBITOR TO EFAVIRENZ

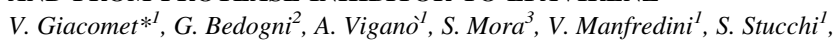
P. Erba ${ }^{I}$, F. Penagini ${ }^{1}$, F. Di Nello ${ }^{I}$, P. Brambilla ${ }^{4}$, G.V. Zuccotti ${ }^{l}$

${ }^{I}$ Pediatric Clinic, L. Sacco Hospital, University of Milan, Milan, Italy;

${ }^{2}$ Clinical Epidemiology Unit, Liver Research Center, Trieste, Italy;

${ }^{3}$ Laboratory of Paediatric Endocrinology, San Raffaele Scientific Institute,

Milan, Italy; ${ }^{4}$ ASL Milano 2, Milan, Italy

Background: No long-term data are available on anthropometric and body compositional changes in HIV-infected children and adolescents switching from stavudine (d4T) to tenofovir (TDF) and from protease inhibitor (PI) to efavirenz (EFV).

Methods: Twenty-four (12 males) HIV-infected children and adolescents aged 5-18 years were switched from a regimen based on lamivudine $+\mathrm{d} 4 \mathrm{~T}+\mathrm{PI}$ to one containing lamivudine $+\mathrm{TDF}$ and EFV. Anthropometry and body composition were evaluated yearly for six consecutive years after the switch. Body composition was assessed using dual-energy X-ray absorptiometry. Outcomes of interest were weight, height, body mass index (BMI), body fat, percent body fat (body fat/body mass), percent arm fat (arm fat/body fat), percent leg fat (leg fat/body fat) and percent trunk fat (trunk fat/body fat). Mixed linear regression was used to model the outcome-time relationships of interest using baseline age and gender as covariates, the patient as random coefficient, and time as random slope. Missing data (11 of 168 time-points) were handled by the mixed model.

Results: All patients maintained the antiretroviral regimen introduced after the switch. HIV-RNA steadily persisted $<50 \mathrm{cp} / \mathrm{mL}$ in most cases; three subjects showed transitory HIV-RNA blips $(73-456 \mathrm{cp} /$ $\mathrm{mL}$ ). Stable CD4 T-cell counts were observed in all cases during the study period. The yearly mean change in weight, height and BMI was $2.5(95 \%$ CI $1.8-3.3) \mathrm{kg}, 0.03(0.02-0.04) \mathrm{m}$, and $0.4(0.2-0.6) \mathrm{kg} /$ $\mathrm{m}^{2}$, respectively. Fat mass increased of a mean of $0.7(0.3-1.0) \mathrm{kg} /$ year, corresponding to $0.5(0.04-1.0) \%$ year of body mass. There was no change in percent arm fat $(0.0,-0.1$ to $0.2 \%$ /year $)$ while percent leg fat decreased of a mean of $-0.7(-1.0$ to -0.3$) \% / y e a r$ and percent trunk fat increased of a mean of $1.5(0.9-2.0) \% / y e a r$.

Conclusions: During a 6-year follow-up, patients who switched from d4T to TDF and from PI to EFV showed anthropometric changes compatible with normal growth and development. In addition, their total and appendicular fat masses were substantially stable during this period.

\section{PP 21}

HIV/HPV CO-INFECTION: MONITORING AND TREATMENT IN SUB-SAHARAN AFRICA: A PUBLIC HEALTH MODEL INTERVENTION

G. Guidotti ${ }^{1}$, C. Moscatelli ${ }^{\prime}$, L. Palombi ${ }^{2}$, M.C. Marazzi ${ }^{3}$, C. Carrilho ${ }^{4}$, E.S.F.

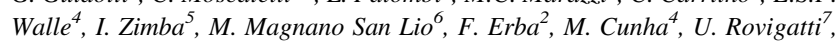
I. Marchetti ${ }^{7}$, M.P. Cioni ${ }^{7}$, G. Bevilacqua ${ }^{7}$

${ }^{I}$ Scuola di Dottorato in Scienze Molecolari-Università di Pisa, Pisa, Italy; ${ }^{2}$ Università Di Roma Tor Vergata, Rome, Italy; ${ }^{3}$ Università LUMSA Facoltà di Scienze della Formazione, Rome, Italy; ${ }^{4}$ Department of Pathology,

Universidade Eduardo Mondlane, Hospital Central de Maputo, Maputo, Mozambique; ${ }^{5}$ DREAM Health Center of Benfica, Maputo, Mozambique; ${ }^{6}$ Community of Sant'Egidio, Drug Resources Enhancement against AIDS and Malnutrition (DREAM), Rome, Italy; ${ }^{10}$ Dipartimento di oncologia, dei trapianti e delle nuove tecnologie in medicina Università di Pisa, Pisa, Italy

Background: The DREAM Program (Drug Resource Enhancement against Aids and Malnutrition) initiated in 2002 by the Community of Sant'Egidio, a faith based organization, is devoted to the treatment and care of HIV/AIDS patients in many African countries with over 100,000 patients in care. Prevention of Mother to Child Transmission (PMTCT) is a main focus of the program $(15,000$ pregnancies followed from 2002 to 2010). We evaluated an approach for control and prevention of cervical cancer in co-infected African women and analyzed the risk of HPV persistence and the presence of cervical lesions, according to HIV status, immunologic baseline parameters and Antiretroviral Treatment (ART).

Methods: As of August 2007 a cohort of HIV positive pregnant women was enrolled at the DREAM Benfica health center, Maputo, Mozambique. This population was monitored for the presence of cancerous and pre-cancerous cervical uterine lesions, using the Bethesda classification (ASCUS, LSIL and HSIL). At present 319 women have been enrolled: 236 HIV positive and 83 HIV negative; mean age was respectively $35.3 \pm 7.7$ and $35.4 \pm 9.9$. At the enrolment Pap smears were performed and delivered to the pathology facility of the Hospital Central de Maputo for cytology examination. Cervical specimens were concurrently sent to the Pathology Institute of the University of Pisa for genetic identification and genotyping of HPV with the Clinical Array test (Genomica SAU). Women with abnormal pap-test were referred for colposcopy and/or biopsy. HSIL lesions were treated as soon as possible with LEEP (loop electrosurgical excision procedure).

Results: Among 236 HIV+ women, 173 (73.3\%) were HPV positive at first screen. 43 of 83 HIV negative patients (51.8\%) were HPV positive. The risk of HPV infection was significantly increased among HIV infected women (RR 1.3; CL 1.1-1.5; p < 0.01). When data were analyzed by $\mathrm{CD} 4$ cell count strata, relative risk increased to 1.6 (CL 1.1-2.5; p < 0.01). Severe lesions (CIN2/CIN3) significantly correlated with CD4 cell counts dichotomized at 200 cells in a logistic binary regression model (forward stepwise) which included HIV status, viral load and CD4 cell counts. The Risk increase in patients with less than 200 CD4 cells was very high (RR 4.5; CL 1.1-20.1). Cox regression confirmed that the main risk factor associated with severe lesions was CD4 cell counts under 200 (RR 4.0, CL 1.1-14.2). Conclusions: Our preliminary data confirms the potential role of ART in protecting against cervical cancer in HIV positive patients. The immune reconstitution mediated by ART enables the increase in CD4 cell count numbers and decreases the risk of cervical cancer development. 


\section{PP 22}

POPULATION-BASED DATABASE TO STUDY THE INCIDENCE OF CANCER IN HIV-INFECTED PATIENTS IN BRESCIA

A. Calabresi ${ }^{\prime}{ }^{1}$, A. Ferraresi ${ }^{1}$, C. Scarcella ${ }^{2}$, R.M. Limina ${ }^{3}$, F. Donato ${ }^{3}$,

A. Vavassori ${ }^{1}$, E. Foca ${ }^{l}$, M. Mendeni ${ }^{1}$, F. Castelnuovo ${ }^{1}$, A. Scalzini ${ }^{1}$,

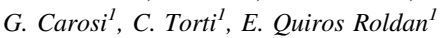

${ }^{1}$ Department of Infectious Diseases, University and Spedali Civili of Brescia,

Brescia, Italy; ${ }^{2}$ Brescia Local Health Authority, Brescia, Italy; ${ }^{3}$ Chair

of Hygiene, University of Brescia, Brescia, Italy

Objective: Individuals who are infected with HIV have an increased risk of cancer. Our objective is to create a population-based database (DB) in Brescia (Northern Italy).

Methods: Information recorded in the clinical DB in use in our Institute (Health\&Notes, Healthware) were integrated with the Brescia Local Health Agency Database (ASL) and the Local Cancer Registry (LCR). The criteria to select cancer cases were: (1) HIV infection diagnosis before cancer; (2) cancer diagnosis between 1999 and 2009.

Results: A total of 421 cancer cases (belonging to 391 HIV infected patients) were confirmed through cross-check among the 3 databases. Three-hundred and thirty-one (78\%) cases were already recorded in our DB, while further 90 cases were obtained from ASL and/or LCR. They occurred mainly in males $(\mathrm{n}=316,81 \%)$. Mean age at first cancer diagnosis was 45 years (SD: 10.5) and was higher in men than women $(\mathrm{P}<0.001)$. Table 1 shows cancer diagnoses. Interestingly 7\% patients had multiple cancers. Among these patients, two had three cancers: both had Kaposi sarcoma associated, in the first patient, with papillary thyroid carcinoma + squamous skin cell carcinoma and, in the second, with intestinal squamous cell carcinoma and nonHodgkin lymphoma.

Table 1

\begin{tabular}{lrr}
\hline Cancer classification, ICD-10 & N. & \multicolumn{1}{c}{$\%$} \\
\hline $\begin{array}{l}\text { C00-14, C30-32; Malignant neoplasms of lip, oral } \\
\quad \text { cavity and pharynx }\end{array}$ & 6 & 1.42 \\
C16; Malignant neoplasm of stomach & 6 & 1.42 \\
C18-20; Malignant neoplasm of colon, & 8 & 1.90 \\
$\quad$ rectosigmoid junction and rectum & & \\
C21; Malignant neoplasm of anus and anal canal & 5 & 1.19 \\
C22; Malignant neoplasm of liver and intra- & 34 & 8.08 \\
$\quad$ hepatic bile ducts & & \\
C34; Malignant neoplasm of bronchus and lung & 23 & 5.46 \\
C43; Malignant melanoma of lip & 8 & 1.90 \\
C44; Other malignant neoplasms of skin & 43 & 10.21 \\
C46; Kaposi's sarcoma & 96 & 22.80 \\
C50; Malignant neoplasm of breast & 10 & 2.38 \\
C51, 52, 54, 57; Malignant neoplasms of female & 3 & 0.71 \\
$\quad$ genital organs & & \\
C53; Malignant neoplasm of cervix uterus & 9 & 2.14 \\
C60-63; Malignant neoplasms of male genital & 15 & 3.56 \\
$\quad$ organs & & \\
C64-68; Malignant neoplasms of urinary tract & 8 & 1.90 \\
C70-72; Malignant neoplasm of brain and & 2 & 0.48 \\
$\quad$ meninges & & \\
C81; Hodgkin's disease & 31 & 7.36 \\
C82-85; Non Hodgkin's disease & 94 & 22.33 \\
$\begin{array}{l}\text { C90; Multiple myeloma } \\
\text { C91-95; Lymphoid leukaemia }\end{array}$ & 0.48 \\
Others** & 2 & 0.48 \\
\hline & & 3.80 \\
\hline
\end{tabular}

Table 1 continued

\begin{tabular}{lll}
\hline Cancer classification, ICD-10 & N. & $\%$ \\
\hline Total & 421 & 100 \\
\hline
\end{tabular}

Conclusions: Integration between complementary DB's is necessary to provide a complete picture of cancer incidence in HIV patients. The occurrence of multiple cancers in individual patients suggests the importance of studies revealing possible predisposing factors of cocancers in some patients.

PP 23

\section{SWITCHING TO BOOSTED OR UNBOOSTED ATAZANAVIR} IMPROVES GLUCOSE TOLERANCE IN HIV INFECTED SUBJECTS: ATAGLU COHORT STUDY

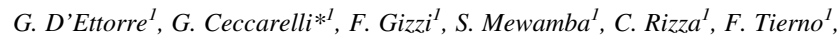
P. Massetti ${ }^{1}$, C.M. Mastroianni ${ }^{1}$, V. Vullo ${ }^{1}$

${ }^{I}$ Department of Public Health and Infectious Diseases, University of Rome "Sapienza", Rome, Italy

Background: The risk of insulin resistance in HIV-infected patients under HAART stems from exposure to the same environmental factors that have led to an increased incidence of these conditions in the general population and from the negative effects on glucose metabolism inherent to components of antiretroviral regimens.

Objective: To characterize the glucidic profile after switching from a Lopinavir/r (LPV/r) containing regimen to an Atazanavir (ATV) based regimen at 24 months.

Methods: ATAGLU is a retrospective cohort study in 198 patients (pt) with undetectable HIV-RNA. We compared pt switching from LPV/r to ATV based regimens (51 ATV/r and 34 ATV unboosted) with those continuing LPV/r containing regimens (pt 113). Outcome measures were 24 months fasting plasma glucose (FPG), insulin levels and homeostasis model assessment-insulin resistance (HOMAIR).

Results: All pt were caucasian with a median age of 46 years (range 25-71); median duration of PI use before switch was 34 months. Median CD4 count was 469 cells $/ \mathrm{mm}^{3}$. Before switching the prevalence of diabetes (fasting glucose $=126 \mathrm{mg} / \mathrm{dL}$ or 2-h glucose $=200 \mathrm{mg} / \mathrm{dL}$ ) among all cohort was $3 \%$ (these pt were excluded from the analysis) and the median of HOMA-IR was 1.9 (range 0.9-3.1) with no difference between the group that changed PI and the group that continued LPV/r. After 24 months from switching we observed a significative reduction of HOMA-IR: the group that continued LPV/r presented a value of 2 while the group under ATV based therapies presented a HOMA-IR of $1.7 \mathrm{p}<0.05$. We did not find any difference related to the association of Ritonavir to Atazanavir (ATV/r 1.8 and ATV unboosted 1.5). No patients from the group receiving ATV with and or without Ritonavir developed impaired glucose tolerance and diabetes mellitus, on the other hand the appearance of these conditions were detected in $9.4 \%$ ( $8 \mathrm{pt}$ ) of patients that continued LPV/r. Moreover we observed a significant decrement of triglycerides (TG) levels and an increase of high-density (HDL) levels.

Conclusion: An increased incidence of insulin resistance, impaired glucose tolerance and diabetes mellitus are usually observed in pt on PI-HAART. Switching from LPV/r to ATV with and or without Ritonavir could improve the glucose metabolism and HOMA-IR after a follow-up of 24 months; in addition the switch could reduce dyslipidemia. Switching from LPV/r to ATV boosted or unboosted could give a long term benefit on glucose metabolism 


\section{PP 24}

TREATMENT SIMPLIFICATION TO ATAZANAVIR/RITONAVIR PLUS LAMIVUDINE IS CORRELATED TO AN INCREASE OF HEALTH-RELATED QUALITY OF LIFE (HRQOL) (ATAZANAVIR AND LAMIVUDINE SIMPLIFICATION STUDY, ATLAS)

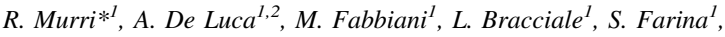
N. Ciccarelli ${ }^{l}$, L. Sidella ${ }^{\prime}$, M. Doino ${ }^{l}$, A. D'Avino ${ }^{l}$, I. Fanti ${ }^{l}$, A. Mondi ${ }^{l}$, R. Cauda ${ }^{I}$, S. Di Giambenedetto ${ }^{I}$

${ }^{1}$ Università Cattolica S Cuore, Rome, Italy; ${ }^{2}$ Siena University Hospital, Siena, Italy; Second Division of Infectious Diseases

Background: ATLAS is a pilot 48 weeks single-arm treatment simplification trial to a 2-drug regimen with atazanavir/ritonavir (ATV/ r) + lamivudine (LAM) in stable virologically suppressed HIV-positive patients on an atazanavir/ritonavir-based three-drug regimen. Impact of simplification on HRQoL is not known.

Methods: Pts on ATV/r + 2NRTI, without previous treatment failure or resistance to PI or LAM, with HIV-RNA $<50 \mathrm{c} / \mathrm{mL}$ for $>3$ months, CD4 $>200$ cells for $>6$ months, HBsAg-negative and ATV plasma levels $>$ efficacy thresholds were eligible. Study was designed to enroll 40 pts. At baseline (BL), regimens were simplified to ATV/r $300 / 100 \mathrm{mg}$ qd + LAM $300 \mathrm{mg}$ qd. Pts were monitored at 4, 12, 24, 36, 48 weeks. Adherence to drugs, belief in therapy, self-reported symptoms and HRQoL were assessed at BL and 48 weeks. A Symptom Score was built summing self-reported scores for each of 19 listed symptoms with range from to 76)

Results: 40 pts enrolled (57\% M, 22\% IDU, 22\% AIDS), with median age 45 years, time since HIV-RNA $<50 \mathrm{c} / \mathrm{mL} 663$ days (IQR 320-895), CD4 count 598 cells $/ \mu \mathrm{L}$ (472-777). 39 pts discontinued tenofovir, 1 abacavir. Data were reported on $38 / 40$ patients who reached 48 weeks. 2/38 pts had virological failure (both without resistance mutations, undetectable plasma ATV and successfully reinduced with 2NRTI). Median CD4 change was +33 cells $/ \mu \mathrm{L}$ $(\mathrm{p}=0.24)$.Six severe adverse events were recorded (4 renal colic, 1 hypertensive crisis, 1 brain hemorrhage). Adherence was stably optimal during the study (83.7 at BL and 82.9 at 48 weeks; $\mathrm{p}=0.85$ ). A significant improvement in total physical $(\mathrm{p}=0.002)$ and mental health scores $(p<0.001)$ was observed but not in the self-reported symptom score $(\mathrm{p}=0.34)$. At multivariable analysis, the change in physical health was independently correlated to physical health at BL (B coeff $-0.78 ; 95 \%$ CI $-1.04 ;-0.53, \mathrm{p}<0.001$ ) and symptom score at BL (B coeff $-0.77 ; 95 \% \mathrm{CI}-1.34 ;-0.22, \mathrm{p}=0.008$ ) while the change in the mental health to age (B coeff $-0.79 ; 95 \% \mathrm{CI}-1.18$; $-0.41, \mathrm{p}<0.001)$ and mental health at BL (B coeff $-0.60 ; 95 \% \mathrm{CI}$ $-0.85 ;-0.34, \mathrm{p}<0.001)$. Change in belief in therapy was significantly correlated to mental health but only at univariate analysis (B coeff $6.23 ; 95 \%$ CI $0.73 ; 11.73, \mathrm{p}=0.03$ )

Conclusion: Simplification to ATV/r + LAM qd was correlated to an increase in both physical and mental health. This effect does not seem mediated by an improvement in the self-reported Symptom score even though the small sample size could not allow definite considerations. Adherence remained optimal over time.

\section{PP 25}

EVOLUTION OF BONE MINERAL DENSITY AND METABOLISM AND OF SUBCUTANEOUS FAT IN PATIENTS ENROLLED IN THE ATAZANAVIR/R + LAMIVUDINE SIMPLIFICATION STUDY

(ATLAS)

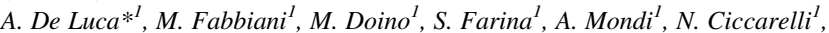

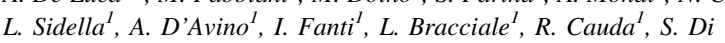
Giambenedetto ${ }^{I}$

${ }^{1}$ Università Cattolica Del Sacro Cuore, Rome, Italy

Background: Reduced bone mineral density (BMD) and lipodystrophy are common in HIV-1-infected individuals and represent a challenge in the clinical and therapeutic management. Different studies found an association between use of tenofovir and bone damage and a higher incidence of fracture.

Methods: In ATLAS, a prospective single-arm pilot study evaluating simplification from $2 \mathrm{NRTI}+\mathrm{ATV} / \mathrm{r}$ to $3 \mathrm{TC}+\mathrm{ATV} / \mathrm{r}$ (Fabbiani et al., XVIII International AIDS Conference 2010, Abstract THLBB207), BMD, limb fat (DXA) and facial adipose tissue (ultrasound) were measured at baseline (BL) and 48 weeks. Additional parameters assessed were demographics, type(s) and duration of prior antiretroviral therapy, lipodystrophy (overall and by region), CD4 counts, fasting metabolic parameters, markers of bone metabolism.

Results: Forty patients were enrolled (57\% M, 22\% IDU, 22\% AIDS), with median age 45 years, time since HIV-RNA $<50 \mathrm{c} / \mathrm{mL}$ 663 days (IQR 320-895), CD4 count 598 cells/mmc (472-777). 39 patients discontinued tenofovir, 1 abacavir. At analysis, 38/40 patients had reached 48 weeks. In 33 evaluable patients the calcium concentration, PTH and vitamin D did not show significant modifications from BL at 48 weeks, whereas significant changes of osteocalcin (mean $-12 \mathrm{ng} / \mathrm{mL} ; \mathrm{p}<0.001)$ and alkaline phosphatase (mean $40 \mathrm{UI} / \mathrm{L} ; \mathrm{p}<0.001)$ were observed. There was an increase of cheek subcutaneous fat $(\mathrm{n}=35$; mean $+0.54 \mathrm{~g} ; \mathrm{p}=0.04)$ and upper limbs $(\mathrm{n}=35$; mean $+145 \mathrm{~g} ; \mathrm{p}=0.003)$, but not lower limbs fat. BMD $(\mathrm{n}=35)$ showed a trend towards an increase in L2-L4 $\left(+0.01 \mathrm{~g} / \mathrm{cm}^{2}\right.$; $\mathrm{p}=0.06$ ) but not in the proximal femurs nor in other districts, where there was no significant change. No patient had had a fracture since being infected with HIV. Osteoporosis $(t$ score $<-2.5$ SD below normal) at BL and 48 weeks was found in $3(7 \%)$ and in $3(8 \%)$ and osteopenia ( $t$ score -1.0 to $-2.5 \mathrm{SD}$ ) in $16(40 \%)$ and $13(38 \%)$, respectively. The only factor associated with osteopenia or osteoporosis (logistic regression) was older age (OR 2.56 per 10 years older; 95\% CI 1.05-6.25; $\mathrm{p}=0.04)$.

Conclusion: The switch from ATV/r +2 NRTI to ATV/r + 3TC was associated with improved bone mineral density and bone metabolism markers and with increased subcutaneous fat in several districts.

\section{PP 26}

ITALIAN SURVEY ON THE DIAGNOSIS OF OSTEOPOROSIS IN HIV: PRELIMINARY RESULTS

F. Vescini ${ }^{1}$, F. Di Campli ${ }^{2}$, M. Borderi ${ }^{3}$, workgroup Italian SorBone ${ }^{4}$ ${ }^{I}$ Centro per la Diagnosi e Cura dell'Osteoporosi, San Donà di Piave, Pavia, Italy; ${ }^{2}$ ViiV Healthcare, Verona, Italy; ${ }^{3}$ Infectious Diseases Unit, Bologna, Italy; ${ }^{4}$ Italian SorBone workgroup, Italy

Introduction: Combined antiretroviral therapy (c-ART) has reduced mortality of HIV infected patients. Due to the prolonged survival many co-morbidities have rose up and a high prevalence of osteoporosis has been reported.

Aim: To investigate medical consciousness of osteoporosis as a comorbidity and the resources commonly used to make the diagnosis.

Methods: The survey has been sent to most of the Italian centres treating HIV infection and 20 of them have filled it up as yet.

Results: A mean of 695 patients are currently treated with c-ART in the surveyed centres. In the first semester of 2010 a mean of 28 naïve patients initiated c-ART in each centre. Eleven $(55 \%)$ centres usually perform a screening for osteoporosis in selected patients. Screenings are significantly more frequent $(p=0.012)$ in the centres following guidelines, and the most used selection criteria are previous fractures (70\%), use of steroids (60\%) and low BMI (45\%). DXA measurement is the preferred diagnostic tool, but $85 \%$ of the centres complains for a difficult access to it. Almost all the centres measure calcium (95\%), phosphate (95\%) and vitamin D (85\%). PTH is dosed in $65 \%$ of the centres while $70 \%$ of them measure at least one marker of bone turnover $(60 \%$ in order to monitor the toxic effect of c-ART and $45 \%$ to evaluate the toxic effect of HIV). The estimation of the relative risk of fracture is chosen by $45 \%$ of the centres, while $15 \%$ of them use markers to choose an appropriate anti-osteoporosis therapy and $30 \%$ 
to monitor the follow up of this treatment. The presence of a diagnosis of osteoporosis drives the choice of c-ART in almost all the centres, both in naïve $(80 \%)$ and in experienced $(85 \%)$ patients. Among comorbidities osteoporosis is perceived as a severe one by the $5 \%$ of the centres, while $30 \%$ consider it a moderate complication and $45 \%$ a mild one.

Discussion: This preliminary results confirm that a pathway dedicated to the diagnosis of osteoporosis is felt as a necessity only by eleven centres and that their attention is driven by the recommendations of guidelines. Although recommended by guidelines and notwithstanding HIV infection is a cause of secondary osteoporosis, the lack of diagnostic tools forces clinicians toward a selection of the patients for DXA screening: only the most severe patients are screened. This selection is performed on the basis of previous fractures, use of steroids and low BMI, but HIV-specific risk are scarcely taken into consideration. Almost all the centres evaluate calcium/phosphate metabolism and vitamin D status. Half of the centres measure bone turnover markers even though their motivation (monitoring the toxic effect of HIV and c-ART) are not those supported by scientific evidence (RR of fracture and therapeutic monitoring). Finally, notwithstanding the growing evidence of a strong association between osteoporosis and both cardiovascular and renal disease in HIV only one centre considers bone disease as a severe co-morbidity.

\section{PP 28}

VITAMIN D3 SUPPLEMENTATION DECREASES THE RISK OF DIABETES MELLITUS AMONG PATIENTS WITH HIV INFECTION

G. Guaraldi ${ }^{1}$, S. Zona ${ }^{l}$, G. Orlando ${ }^{l}$, C. Federica ${ }^{l}$, G. Ligabue ${ }^{l}$,

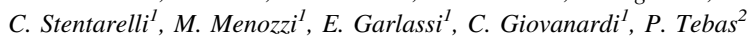

${ }^{1}$ University of Modena and Reggio Emilia, Modena, Italy; ${ }^{2}$ University

of Pennsylvania, Philadelphia, USA

Background: Type 2 diabetes mellitus (DM), insulin resistance and hypovitaminosis D are common among individuals living with HIV. Low vitamin D (VitD) has been associated with insulin resistance and DM in the general population and more recently in the HIV infected population. Our objective was to examine if the use of VitD3 (Cholecalciferol) supplementation could prevent the development of DM among patients with HIV infection.

Methods: We conducted a retrospective study among patients enrolled in the Modena cohort, an ongoing, multicentre, prospective cohort of HIV-infected individuals in care at the Metabolic Clinic of the University of Modena, Italy. All patients seen more than once, without baseline DM were included in this analysis. Incident DM was diagnosed if the fasting plasma glucose was $126 \mathrm{mg} / \mathrm{dL}$ in a subsequent visit, or the primary care provider of the patient had initiated treatment for DM between visits. Individuals were classified as receiving VitD3 supplementation if they reported consistently supplementing their VitD3 intake with 30,000 IU weekly as recommended during each clinic visit. Adjusted hazard ratios of incident DM were estimated using multivariable Cox regression model.

Results: 1,574 patients meet the entry criteria: $65 \%$ male, with a median (IQR) age of 44 years (40-48), a nadir CD4 177 cells $/ \mu \mathrm{L}$ (72-273), current CD4 509 cells/ $\mu \mathrm{L}$ (367-687), 61\% undetectable HIV RNA, duration of HIV infection 174 months (118-232). 232 (14.7\%) of them reported consistent VitD3 supplementation. The median follow up was 2.4 years (1.3-3.5). There were 74 cases of incident DM, 2 among individuals supplementing VitD3 (0.37 events per 100 PYFU) and 72 among the 1,342 subjects not using VitD3 (2.16 events per 100 PYFU) $(\mathrm{p}=0.003)$. Baseline HOMA-IR (HR 1.05, 95\% CI 1.02-1.08); Age (HR 1.08, 95\% CI 1.05-1.12), baseline VitD (HR 0.96, 95\% CI 0.93, 0.99), and VitD3 supplementation (HR $0.17,95 \%$ CI $0.04-0.72$ ) were independently associated with DM in a model that evaluated also gender, current and nadir CD4 cell count, BMI, girth circumference, triglycerides and HIV undetectability.
Conclusions: The data from this retrospective cohort study suggest that VitD3 supplementation decreased the risk of DM in patients with HIV infection. Vitamin D supplementation may prevent or delay the onset of DM and decrease the incidence of cardiovascular disease in patients with HIV infection. Prospective controlled trials will be needed to confirm this observation.

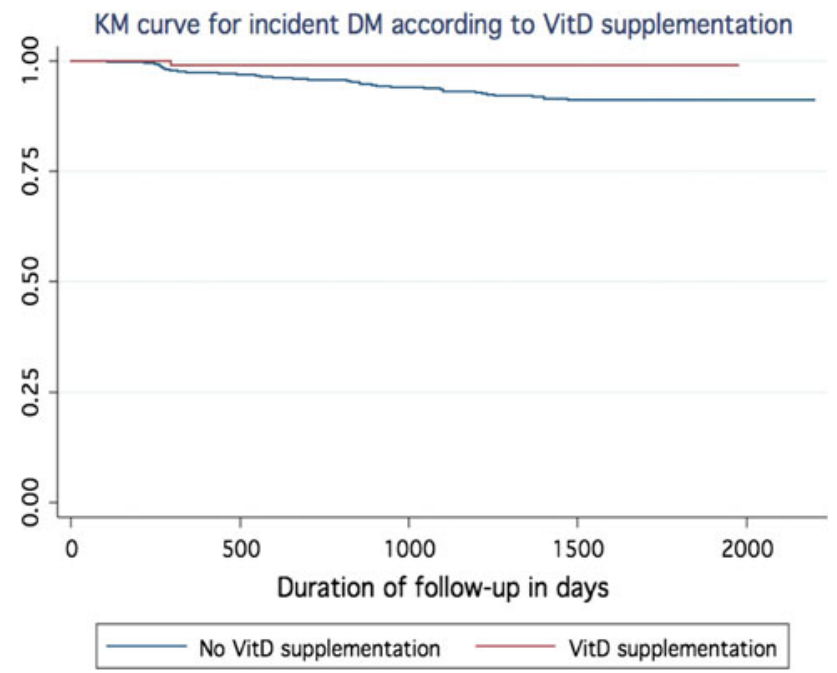

\section{BASIC SCIENCES}

\section{PO 01}

OCCULT HEPATITIS B INFECTION (OBI) IN HIV-INFECTED PATIENTS IN PALERMO, ITALY: PRELIMINARY DATA

P. Di Carlo $^{*^{1}}$, G. Mazzola ${ }^{I}$, D. Ingrassia ${ }^{1}$, G. Guadagnino ${ }^{I}$, F. Vitale $^{l}$, F. Tramuto ${ }^{1}$

${ }^{I}$ Dipartimento di Scienze per la Promozione della Salute, Università degli Studi di Palermo, Palermo, Italy

Background: The prevalence of Occult hepatitis B infection (OBI) showed variability in diverse cohorts among HIV-infected patients. The diagnosis depends on and relative sensitivity of both HBsAg and HBV DNA assays. It also depends on the prevalence of HBV infection in the population.

Objectives: Here, we estimated the prevalence of HIV-infected adults with negative serum $\mathrm{HBsAg}$ and detectable plasma $\mathrm{HBV}$ DNA in Palermo, Italy. Risk factors, and clinical significance of occult HBV in HIV-infected patients and to evaluate the effect of HAART.

Materials and methods: The sample population $(\mathrm{n}=280)$ was negative serum HBsAg HIV-1 infected and highly active antiretroviral therapy-naïve patients. Samples were tested for HBV DNA using three in-house nested-PCR amplification assays to detect HBV preS-S (S), precore-core (C), and Pol viral regions, respectively, "occult" HBV sequence. The analysis was performed at Laboratory of Molecular Epidemiology, Hygiene Section, Department for Health Promotion, University of Palermo.

Results: We report the preliminary data of 180 out $280(64 \%)$ enrolled patients. The median age was $45.5 \pm 9.5$ (range 20-75) years. $87 \%$ were born in Italy and $63 \%$ were male. Median CD4+ count was 329 (range 0-900) cells $/ \mathrm{mm}^{3}$. Sixty-nine percent of the subjects had HIV RNA titers undetectable $(<20$ copies $/ \mathrm{mL})$. Seventy-nine percent showed lamivudine (3TC) or emtricitabine (FTC) plus tenofovir disoproxil fumarate (TDF) regimes. HCV was 
present in $30 \%$ of subjects. Overall, $44.6 \%$ of subjects demonstrate past $\mathrm{HBV}$ infection and $30 \%$ had received prior vaccination. The presence of "occult" HBV sequences was detected in 5 out 180 sera $(2.77 \%)$. The "OBI positive patients" were anti HBs, anti-HBc negative and showed a history of failed vaccination. 4/5 were antiHCV negative and Lam-experienced.

Discussion: In conclusion, the prevalence of occult HBV in this HIV positive study population is lower than that reported by Vitale in non$\mathrm{HIV}$ infected, anti-HBc positive population in the same geographical area but lower than that would have been predicted based on previous studies. The anticore positivity was not a relevant condition for HBVDNA detection. Patients with occult HBV infection belong to an age group that excluded them from previous vaccination programs and are subject who failed following vaccination

\section{PO 02}

\section{PREVALENCE AND TYPE SPECIFIC PAPILLOMAVIRUS LOAD} IN HIV-POSITIVE WOMEN ANAL INFECTION

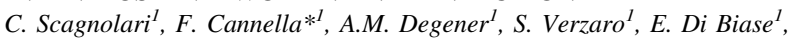
C. Selvaggi ${ }^{l}$, M. Indinnimeo ${ }^{2}$, V. Vullo $^{3}$, G. D'Ettorre ${ }^{3}$, A. Pierangeli $^{l}$, G. Antonelli ${ }^{1}$

${ }^{\prime}$ Deparment of Molecular Medicine 'Sapienza' University, Rome, Italy;

${ }^{2}$ Chirurgic Department "Pietro Valdoni” 'Sapienza' University, Rome, Italy; ${ }^{3}$ Department of Public Health and Infectious Diseases 'Sapienza' university, Rome, Italy

Human Papillomavirus (HPV) infection has been recognized as strongly associated to anal as well as to cervical carcinoma; however, to date, much less is known about factors influencing anal HPV persistence and progression. Anal cancer rates in human immunodeficiency virus (HIV)-positive individual have continued to increase over the past decade, despite the widespread use of highly active antiretroviral therapy (HAART). The risk of development of anal carcinoma in HIV-positive women has been estimated to be at 5-8 times higher than that of HIV-negative women. Even so, the role of HPV-type specific infection and viral load in anal lesions in women have been addressed in a few studies. Hence, the aim of this study was to monitor HIV-positive women anal infections, determining the prevalence of HPV genotypes in anal and cervical brushings and related type-specific HPV loads. Anal brushings were collected in a proctology clinic (Umberto I University Hospital, Rome) from 18 HIV-positive women: a cervical brushing was taken as well. The presence of specific HPV genotypes in anal and cervical brushings was determined by two different PCR assays followed by sequencing, a method that allows the identification of a wide range of HPV types. Type-specific viral load was measured using a quantitative real-time PCR fluorogenic assay with TaqMan probes and primers designed for 14 HPV genotypes in the E6 genomic region. The prevalence of HPV infection in anal samples were higher than in cervical samples (28 vs. $11 \%)$. HPV genotypes detected in anal samples were: HPV 6 (2 cases), HPV 16, HPV 31 and HPV 62 (1 case each), and in cervical samples HPVs 6, 54 and 55 (1 case each). Considering the women with concurrent anal and cervical HPV infection, the simultaneous presence of the same genotype occurred only in 1 out of 3 women. HPV-type specific DNA load was determined in most samples: HPV number of copies was not related to HPV type of risk (low/high). In cervical HPV-negative samples corresponding to an anal sample resulted HPV-positive, measurement of the HPV load of the genotype infecting the anal site gave negative results. Our study showed a high prevalence of HPV anal infection in HIV-positive female patients. Anal infection without concomitant cervical infection suggested different routes of acquisition or different rates of persistence. The frequency of high-risk HPVs in anal samples supported the need to develop HPV screening programs in anal brushings from HIV-positive women.
PO 03

DELAYED MATURATION OF IGG ANTI-HIV AVIDITY IN PATIENTS WITH PRIMARY/ACUTE HIV INFECTION TREATED WITH A RALTEGRAVIR: HAART BASED REGIMEN

G. Orofino ${ }^{*}$, V. Ghisetti ${ }^{2}$, S. Carosella ${ }^{1}$, M. Guastavigna ${ }^{1}$, M. Farenga $^{l}$,

S. Faraoni ${ }^{2}$, T. Allice ${ }^{2}$, P. Caramello ${ }^{I}$

${ }^{I}$ Div.A Malattie Infettive, Ospedale Amedeo di Savoia, Turin, Italy;

${ }^{2}$ Dipartimento di Malattie Infettive, S.C. Microbiologia e Virologia, Ospedale Amedeo di Savoia, Turin, Italy

Background: One of the potential benefits supporting the rationale for the administration of HAART in primary/acute HIV infection is to preserve HIV specific immune function. At this purpose, the role of integrase inhibitors is under investigation. In this study, we evaluated a Raltegravir-HAART based regimen in primary/acute HIV infection and its impact with the maturation of specific IgG anti-HIV avidity in patients with recent HIV infection.

Methods: From 02/2009 to 06/2010, we identified 14 patients (11 males, 3 females, median age 36.4 years; risk factors: 9 MSM, 1 IVDU, 4 eterosexual) with a diagnosis of primary/acute HIV infection (Acute Cohort). HAART ( 2 NRTI +1 boosted IP) was immediately started in 12 of them and planned for 1 year. Raltegravir was added in 9 patients as induction for 12 weeks, then stopped. Anti HIV IgG avidity (Architect HIV Ag/Ab combo, Abbott, Il, US) was performed at time 0 , and then at $1,3,6,9$ and 12 months.

Results: At baseline the average CD4 count was 581 cells/mmc; 6/14 patients had HIV-RNA levels $>100.000$ copies $/ \mathrm{mL}$. Three patients discontinued therapy (1 pregnancy, 2 low adherence). Eight out of nine patients on Raltegravir induction reached a follow-up of at least 9 months. In these patients, HIV-RNA became undetectable $(<50$ copies $/ \mathrm{ml})$ at month 1 in four of them, at month 3 in two and at month 5 in other two patients. In 7/8 patients no maturation of IgG anti-HIV avidity index (AI) was observed: mean AIs at baseline (9 patients), at month 1 (3 patients), 3 (9 patients), 6 (8 patients), 9 (8 patients) and 12 (4 patients) were $0.22,0.26,0.51,0.54,0.43,0.66$, respectively. Only in one patient the AI reached 1.0 at month 4 of follow-up. The average CD4 count at month 1, 3, 6, 9 and 12 was 734, 776, 797, 821, 814/mmc.

Conclusions: A Raltegravir-HAART based regimen in patients with primary/acute HIV infection is highly effective (70\% of undetectable HIV RNA by month 3), but interference with the maturation of specific IgG anti-HIV avidity is observed. Further evaluations are required, but a selective action of Raltegravir on $\mathrm{B}$ cells antibody production during the HAART-induction phase in the early stages of HIV infection can be postulated.

\section{PO 04}

RELATIONSHIP BETWEEN REGULATORY T CELLS, VIRAL LOAD AND CHRONIC IMMUNE ACTIVATION IN HIV-1 INFECTED CHILDREN

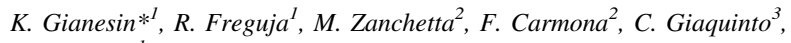
A. De Rossi $i^{1}$

${ }^{I}$ Dipartimento di Scienze Oncologiche e Chirurgiche, AIDS Reference Centre, Università degli Studi di Padova, Padua, Italy; ${ }^{2}$ Istituto Oncologico Veneto, IRCCS, Padua, Italy; ${ }^{3}$ Dipartimento di Pediatria, Università degli Studi di Padova, Padua, Italy

Background: The function of CD4 T cells with regulatory activity (Tregs) is down-regulation of immune responses. This suppressive activity may limit the magnitude of effector responses, with consequent failure in controlling HIV-1 infection, but also may influence chronic immune activation, a hallmark of disease progression. This is the first study that focused on the relationship between viral load, immune activation and Tregs in HIV-1 infected children.

Methods: 89 HIV-1-infected children (6-14 years old) under antiretroviral therapy and $10 \mathrm{HIV}-1$-uninfected age-matched children, 
born to HIV-1-seropositive mothers, were studied. Peripheral blood mononuclear cells (PBMC) were analyzed by four-colour flow cytometry. PBMC were stained with monoclonal antibodies (CD3 FITC, CD38 PE, CD4 PerCP, CD8 PerCP, CD45RA APC) to evaluate $\mathrm{T}$ cell subsets. Treg cells were evaluated using a combination of markers (FoxP3 FITC, CD25 PE, CD4 PerCP, CD127 APC). HIV-1 plasma viremia was determined by COBAS Taqman HIV-1 test. HIV1 DNA levels in PBMC were measured by real-time PCR and expressed as HIV-1 DNA copies/106 CD4 T cells. Statistical analyses were performed using SPSS v17; comparisons between groups were explored using non-parametric tests (Mann-Whitney or KruskalWallis) and correlations were made by the Spearman's rho test.

Results: HIV-1 infected children were divided into subgroups according to HIV-1 plasma viremia: group $1(\mathrm{n}=49$, HIV-1 RNA $<50$ copies/ml); group $2(\mathrm{n}=19$ with HIV-1 RNA from 50 to $1,000$ copies $/ \mathrm{ml})$ and group $3(\mathrm{n}=21, \mathrm{HIV}-1$ RNA $>1,000$ copies/ $\mathrm{ml}$ ). Group 3 had lower CD4 T cells (median 718 [interquartile range 590-1,029] cells/ $\mu \mathrm{l})$ than group 2 (752 [557-1,261] cells/ $\mu 1)$, group 1 (908 [736-1,191] cells/ $\mu \mathrm{l})$, and HIV-1 uninfected children $(1,155$ [768-1,296] cells $/ \mu \mathrm{l})$; overall $\mathrm{p}=0.048$. Conversely, the levels of CD8 T cells were higher in HIV-1 infected than HIV-1 uninfected children $(\mathrm{p}=0.030)$; the expansion was mostly due to the memory CD8CD45RA-cell subset $(\mathrm{p}=0.009)$. Moreover, results showed that the number of activated CD8CD38+ $\mathrm{T}$ cells increased along with HIV-1 plasma viremia $(r=0.403, p<0.0001)$. The proportion of Tregs positively correlated with HIV-1 plasma viremia $(r=0.323, p=0.002)$, while inversely correlated with CD4 T cells $(\mathrm{r}=-0.312, \mathrm{p}=0.004)$, suggesting a selective expansion along with increased viremia and CD4 T cells depletion. Interestingly, a positive correlation was found between CD8CD38+ activated cells and Tregs, considering both cell number $(\mathrm{r}=0.305, \mathrm{p}=0.005)$ and percentage $(r=0.220, p=0.040)$. Furthermore, the proportion of Tregs tended to correlate with HIV-1 DNA load $(r=0.224$, $\mathrm{p}=0.062$ ).

Conclusions: These findings suggest that chronic immune activation persists in children with poor virological response to antiretroviral therapy and may contribute to the expansion of Treg cells. In turn, the suppressive activity of Tregs may impair effector responses against HIV-1 but appears to be ineffective in limiting immune activation.

\section{PO 07}

LONG TERM IMMUNE RECONSTITUTION IN HIV-POSITIVE RELAPSE/REFRACTORY LYMPHOMA PATIENTS AFTER AUTOLOGOUS STEM CELL TRANSPLANTATION

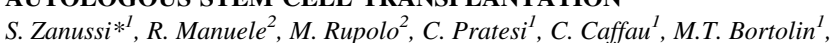

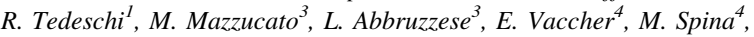
U. Tirelli, G. Basaglia ${ }^{1}$, M. Michieli ${ }^{2}$, P. De Paoli ${ }^{5}$

${ }^{1}$ Microbiology, Immunology, Virology, Oncology Reference Centre, Aviano, PN, Italy; ${ }^{2}$ Cellular Therapy and High-Dose Chemotherapy Unit, Oncology Reference Centre, Aviano, PN, Italy; ${ }^{3}$ Stem Cell Collection and Processing Unit, Oncology Reference Centre, Aviano, PN, Italy; ${ }^{4}$ Division of Medical Oncology A, Oncology Reference Centre, Aviano, PN, Italy; ${ }^{5}$ Scientific Directorate, Oncology Reference Centre, Aviano, PN, Italy

Objectives: To assess immune recovery in long term survivors relapse/refractory HIV-positive (HIV+) lymphoma patients (pts) submitted to Autologous Stem Cell Transplantation (ASCT). To correlate immune recovery with virological and clinical parameters. Methods: HIV+ pts who underwent ASCT at the National Cancer Institute (Aviano, Italy) and with at least 5 years of follow-up after transplantation were included in this retrospective immunological study. CD4, CD8 absolute T lymphocyte subset counts were evaluated by flow cytometry (EPICS XL-Beckman-Coulter). Thymic regeneration was evaluated by assessing the number of sjTRECs per $10^{6}$ peripheral blood mononuclear cells (PBMCs) using a real-time polymerase chain reaction (PCR) quantitative technique. The HIV RNA level was quantified by using the Versant HIV-1 RNA 3.0 assay kit (bDNA; Bayer Diagnostics).

Results: Ten relapse/refractory HIV+ lymphoma pts were studied: 8 had 60, 7 seventy-two, 384 and 396 months follow-up from ASCT. Pre-transplant median CD4 T lymphocyte levels were 191 cells $/ \mu \mathrm{L}$ (range 13-460), a significant increase was observed by month 24 after ASCT (median 300 cells $/ \mu \mathrm{L}$, range: $87-628, \mathrm{p}=0.04$ ) and at least a doubling of all the pre-transplant values at month 72 (median 586 cells $/ \mu \mathrm{L}$, range $379-1,028)$. Six pts reached CD4 T cell levels within a normal laboratory range $(480-1,315$ cell $/ \mu \mathrm{L})$ during a median follow-up of 36 months (range 24-72 months), 50\% being admitted to salvage therapy with CD4 T cell levels below 200 cells/ $\mu \mathrm{L}$. CD8 T lymphocyte counts were expanded in the majority of the pts, and ratio of CD4 cells to CD8 appeared below one for the entire follow-up, except in two pts. sjTRECs $/ 10^{6}$ PBMCs values increased significantly between pre- and post-ASCT $(\mathrm{p}<0.01)$ and in two pts reached the normal range correlated to the respective age group at month 24 and 48. All pts were pre-treated with HAART based on their clinical antiretroviral therapy histories and/or the HIV genotypic test result for a median of 38.6 months (range 8-86) before enrolment. For four pts HAART was changed during ASCT protocol to reestablish its best efficacy. All but one pt showed HIV RNA levels below the detection limit ( 50 copies $/ \mathrm{mL}$ ) during the long term followup post-ASCT.

Conclusions: relapse/refractory HIV+ lymphoma pts with long term follow-up post ASCT show a very good immune and thymic function recovery. Response to HAART is important, but, perhaps, not the unique favourable determinant.

\section{PO 08}

PERIPHERAL BLOOD T REGULATORY CELLS AND TH17 CELLS AND THEIR GENES EXPRESSION PROFILE IN HIV-1 INFECTED PATIENTS AND HEALTHY CONTROLS

M. Tarkowski ${ }^{l}{ }^{1}$, P. Lewkowicz ${ }^{2}$, E. Jablonowska ${ }^{2}$, D. Misciniagi ${ }^{I}$, A. Riva ${ }^{l}$, M. Galli ${ }^{1}$

Universita degli Studi di Milano, Milan, Italy; ${ }^{2}$ Medical University of Lodz, Lodz, Poland

Background: T regulatory cells are an important subpopulation of lymphocytes that mediate suppression of immune reactions, whereas Th17 cells mediate processes of immune activation and both participate in immune responses to HIV infection. The imbalanced participation of these cells during HIV infection may be an important factor determining the pathogenesis of the disease and affecting its course. Our previous analyses have shown the lack of differences in number of T reg cells in peripheral blood of HIV Progressors and LTNP, in the expression of CXCR4 on their membrane, activity, and no association with viral load.

Methods: We enrolled HIV infected patients, naive to ART with progressive infection, long-term non progressors (LTNP) and healthy controls. Analyses of Treg and Th17 cells in peripheral blood were performed by antibody staining and cytofluorimetry. Expression of array of genes in peripheral blood mononuclear cells was performed by use of commercially available PCR Array kit simultaneously analysing 84 different genes included in array (Qiagen, Th17 kit). The PCR reaction was performed on Real Time PCR (Applied Biosystems).

Results: We found that in contrast to healthy control group, progressors had lower number of peripheral blood Treg cells accompanied by higher percentage of Th17 cells and higher density of CXCR4 receptor on CD4+ CD25- T cells. The expression of CCR4, CCR7, CD62L on Treg cells and the status of their activation (CD45RA and RO) was not different between healthy controls and HIV infected patients. Analyses of array of genes involved in Treg/ Th17 differentiation did not show differences between LTNP and 
progressors but in several genes between progressors and healthy controls. In peripheral blood, mononuclear cells of progressors had significantly higher levels of expression of CEPB, IL-1b, IL-8, ISG20, SOCS3, and TNF, and lower expression of CD40L in comparison to healthy controls.

Conclusions: The number of Treg cells but not selected characteristics of these cells are significantly associated with more severe progression of HIV infection and their lower number in peripheral blood in comparison to healthy controls might be due to high accumulation in lymphoid tissues and/or higher susceptibility to HIV cell death. Lower number of Treg cells might contribute to higher immune activation status found in progressors as seen by high number of Th17 cells and expression of several genes that were significantly upregulated or downregulated only between progressors and healthy controls. We conclude that decrease in number of peripheral blood Treg cells concomitant with increased numbers of Th17 cells might be observed only in patients whose HIV progresses rapidly. Such a changes are not apparent in LTNP and distinguishable from healthy controls thus making difficult to conclude about the kinetics of imbalance between immunosuppression and immunoactivation mediated by these two cell subpopulations.

\section{PO 09}

\section{INFLUENCE OF DIFFERENT ART REGIMENS ON IMMUNE} PARAMETERS AND ACTIVATION: ABACAVIR, TENOFOVIR AND RALTEGRAVIR-BASED REGIMENS AND NK CELLS

F. Bozzano ${ }^{\prime}$, F. Marras ${ }^{2}$, A. Beltrame ${ }^{3}$, A. Alessandrini ${ }^{3}$, G. Pagano $^{3}$, A. De Maria ${ }^{2}$

${ }^{1}$ Istituto G. Gaslini, Genoa, Italy; ${ }^{2}$ Università degli Studi di Genova, Genoa, Italy; ${ }^{3}$ Azienda Ospedaliera, Ospedale S. Martino, Genoa, Italy

Background: antiretroviral treatment (ART) has been shown to interfere with several parameters involved in homeostasis of metabolic and inflammatory pathways, as well as with cell proliferation. Innate immune function may be variably influenced by antiretrovirals targeting different viral and metabolic pathways. We studied peripheral NK cells in patients on different ART regimens to understand their possible modulation of the immune response in vivo. An in vitro model was developed to verify correlates of activity.

Patients and methods: patients on successful treatment with ART regimens (VL $<50 \mathrm{cp} / \mathrm{ml}$ for at least 12 months) were enrolled. Raltegravir (RAL)-, Abacavir (ABC)- or Tenofovir (TDF)-containing triple-drug regimens were considered for patients on PI treatment. NK cell function and phenotype were studied by flow-cytometry. In vitro culture of healthy donor NK cells in the presence of corresponding regimens were evaluated.

Results: Patients (no. 24) falling into the 3 treatment groups were enrolled. All but 1 patient in each group had suppressed VL at the time of analysis. Mean CD4+ cell counts were comparable in ABC and TDF and were lower in RAL patients $(21.56 \pm 11.1$; $24.54 \pm 20.09 ; 18 \pm 5.19)$ respectively. Among patients in $\mathrm{ABC}$ and TDF, NKp46 (39.02 $\pm 11.65 ; 46.82 \pm 24.06$, respectively), NKp30 $(8.67 \pm 6.47 ; 9.88 \pm 7.15$ respectively), NKG2D $(49.31 \pm 17.34$; $48.17 \pm 26.44$, respectively) and DNAM-1 (74.94 \pm 10.08 ; $80.07 \pm 5.67$ respectively) activating NK cell receptor expression was comparable. Non-KIR inhibitory receptors (CD85j, NKG2A/ CD94) were comparable in patients belonging to the two groups. Evidences for increased NK cell activation (HLA-DR expression) and decreased cytotoxicity and IFN $\gamma$ production were observed among patients in TDF compared to ABC patients. Patients in RAL group had more advanced disease according to $\mathrm{CD} 4+$ proportions and absolute counts, as expected. However, they had the least activation of NK cells (as defined by HLA-DR expression), when compared to patients in $\mathrm{ABC}$ or TDF groups $(27.46 \pm 18.19 ; 31.62 \pm 12.53$;
$45.67 \pm 11.21$ respectively). Reduced proportions of activating receptor expression were also recorded in this group (NKp30: $0.75 \pm 0.41 ; \quad$ NKG2D: $27.28 \pm 20.5$, DNAM-1: $50.18 \pm 21.15$ ), when compared to the other patients. In addition, they showed decreased inhibitory receptor expression on NK cells (CD85j, NKG2A/CD94) compared to the other patients.

Conclusion: These preliminary data suggest that among stably treated patients on different ART regimens, antiretroviral drugs may exert different immunomodulating effects in addition to their primary antiretroviral activity. Effects on NK cells may affect the antigen presentation and adaptive ( $\mathrm{T}$ and $\mathrm{B}$ cell) immune response to HIV itself and other recall antigens, as well as to new antigens (e.g. vaccination, tumor). Once confirmed and extended on larger patient groups, knowledge of ART effects on innate immune mechanisms may be suitably exploited in HIV patients according to clinical

\section{PO 10}

LONG TERM CD4 RECOVERY IN FULLY SUPPRESSED SUBJECTS TREATED WITH FIRST LINE HAART IS INFLUENCED BY TIME TO REACH UNDETECTABILITY

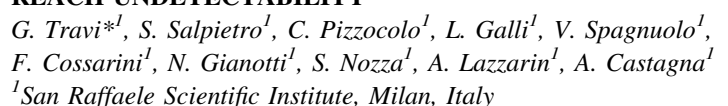

Background: We evaluated if factors related to viral dynamics (i.e. time to reach first HIV-RNA $<50 \mathrm{cps} / \mathrm{mL}$ and blips occurrence) may influence the magnitude of long-term CD4 recovery in fully suppressed subjects treated with first line HAART.

Methods: Observational study from IDD-HSR database. We included HIV+ subjects treated with first line HAART, from 1996 to 2007, who maintained viral suppression for at least 2 years without regimen modification. Viral blip (VB) was defined as any value of HIV-RNA $>50 \mathrm{cps} / \mathrm{mL}$ during first HAART, not subsequently confirmed. Baseline (BL) was defined as start date of HAART and end of follow up (FU) as the date of last HIV-RNA $<50 \mathrm{cps} / \mathrm{mL}$ before treatment discontinuation or last visit. Results as median (IQR). Linear regression within each subject was applied to estimate the trend of CD4 values (slope per year). Generalized linear model (GLM) applied at multivariable analysis with CD4 slope as outcome.

Results: 255 subjects met the inclusion criteria: they had a median age of $41(38-48)$ years, $210(82 \%)$ were males and $133(52 \%), 104$ $(41 \%)$ and $18(7 \%)$ started respectively a PI-based or NNRTI-based or NRTI-based regimen. VB was observed in 119 (47\%) subjects. Subjects with or without VB did not differ for gender, type of HAART regimen, HIV risk factor, previous AIDS defining events, HCV or HBV co-infection. First HAART duration was higher among subjects with VB [4.5 (3.3-5.6) vs. $3.8(2.8-4.5)$ years, $p=0.006]$. Subjects with or without VB had similar baseline CD4 cells count and viral load (VL) [CD4: $p=0.324 ; \log _{10}$ VL: $p=0.889$ ]; also CD4 recovery (slope) was similar between the two groups $(\mathrm{p}=0.772)$. Time to reach first HIV-RNA $<50 \mathrm{cps} / \mathrm{mL}$ was similar in both groups, [VB: 4.9 (3.1-6.5) months; no VB: 5.0 (3.1-8.4) months, $p=0.448$ ] but time spent with negative VL was significantly different [VB: 50 (34-70) months; no VB: 43 (28-56) months, $p=0.001$ ]. At GLM, CD4 slope was significantly related to time spent with negative VL $(\beta=-3.7, \mathrm{p}<0.0001)$, to time to get undetectable VL $(\beta=-6.1$, $\mathrm{p}=0.042)$ and to first HAART duration $(\beta=27.1, \mathrm{p}=0.011)$. No influence of age, sex, HIV risk factor, years since HIV infection, occurrence of VB, type of HAART, BL CD4, CD8, $\log _{10} \mathrm{VL}$ and CD4 gain obtained between start date of HAART and first undetectable VL.

Conclusions: In fully suppressed HIV+ subjects treated with first line HAART, CD4 recovery is inversely related to time to reach undetectability. Occurrence of VB did not influence CD4 recovery. 


\section{PO 11}

PHARMACOLOGICAL MODULATION OF APOPTOSIS OF PBMC BY DARUNAVIR AND MARAVIROC

I. Sauzullo ${ }^{I}$, F. Mengoni ${ }^{I}$, A. Ermocida ${ }^{I}$, R. Rossi ${ }^{I}$, M. Lichtner $^{2}$,

A.P. Massetti ${ }^{l}$, C.M. Mastroianni ${ }^{2}$, V. Vullo ${ }^{l}$

${ }^{I}$ Sapienza Università di Roma, Rome, Italy; ${ }^{2}$ Sapienza Università, Latina, Polo Pontino, Italy

Background: HIV infection causes a progressive depletion of CD4+ $\mathrm{T}$ cells probably due to accelerated apoptosis. The HIV protease inhibitors, in addition to direct anti-viral effect, may also modulate apoptosis. CCR5 antagonist may exhibit immunomodulant properties beyond their capacity to inhibit HIV entry. In the present study, we explored the in vitro effect of HIV protease inhibitor darunavir (DRV) and CCR5 antagonist maraviroc (MVC) on PBMC by analyzing apoptosis and cell viability.

Methods: PBMC were isolated from healthy donors. Cells $(1 \times 106 /$ $\mathrm{ml})$ were incubated in presence of medium alone or DRV $(0.1-1-$ $10 ? \mathrm{~g} / \mathrm{ml})$ or DRV in combination with MVC $(10 \mu \mathrm{M})$ at $37^{\circ} \mathrm{C}$ under $5 \% \mathrm{CO}_{2}$, and after $24 \mathrm{~h}$ of incubation the pro-apoptotic effect was measured. The anti-apoptotic effect was measured inducing the apoptosis with protein synthesis inhibitor puromycin (PMC $10 \mu \mathrm{g}$ / $\mathrm{mL}$ ). Apoptosis rate was assessed morphologically using fluorescent dyes that intercalate DNA: acridine-orange/ethidium bromide. The results were as percentage of apoptotic cells.

Results: At $0 \mathrm{~h}$ the PBMC showed no morphological evidence of apoptosis and the viability was $>95 \%$ for all condition. The treatment of cells with high concentration of DRV (10 ?g/ml) for $24 \mathrm{~h}$ did not exert any significant pro-apoptotic effect if compared to control (DRV $=22.6 \%$ vs. $\mathrm{CNT}=23 \%$ spontaneous apoptosis $)$. In addition, a significant effect of DRV was seen for PBMC in the modulation of apoptosis exogenously induced with the stressing agents puromycin $(\mathrm{CNT}=60 \% ; \quad$ DRV $0.1 ? \mathrm{~g} / \mathrm{ml}=42 \% ; \quad 1 ? \mathrm{~g} / \mathrm{ml}=35 \% ; \quad 10 ? \mathrm{~g} /$ $\mathrm{ml}=41 \%)$. We found that DRV exerted anti-apoptotic activity, that was not dose-dependent. Interestingly, the effect of DRV as antiapoptotic agent was similar to that of HIV protease inhibitor IDV, which at the concentration of $100 \mathrm{nM}$ reduced apoptosis to a very similar extent (39\%). In presence of MVC $(10 \mu \mathrm{M})$ plus DRV (0.1-1$10 ? \mathrm{~g} / \mathrm{ml}$ ), the percentage of apoptosis of PBMC was 33.5, 36 and $36,5 \%$, respectively. MVC did not affect anti-apoptotic activity of DRV.

Conclusions: The study of extravirologic properties of newer antiretroviral drugs represents an area of active investigation. Given the effects of DRV (anti-apoptotic activity) and MVC (down-modulation of chemotaxis), our in vitro findings encourage studies assessing the impact on immunologic response of regimens including these two antiretroviral drugs.

\section{PO 12}

EVALUATION OF CELLULAR ASSOCIATED HIV-1 DNA LEVELS IN HIV-1 INFECTED PATIENTS RECEIVING A LONG-TERM HIGHLY ACTIVE ANTIRETROVIRAL THERAPY

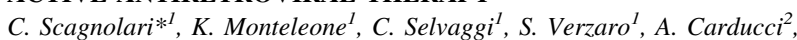
F. Ceccherini-Silberstein ${ }^{3}$, M. Andreoni ${ }^{4}$, G. Antonelli ${ }^{1}$, E. Riva $^{2}$ ${ }^{\prime}$ Department of Molecular Medicine, Laboratory of Virology "Sapienza" University of Rome, Rome, Italy; ${ }^{2}$ Microbiology and Virology Dept, University Campus Bio-Medico, Rome, Italy; ${ }^{3}$ Department of Experimental Medicine, University of Rome Tor Vergata, Rome Italy; ${ }^{4}$ Clinic Infectious Diseases, Clinic Infectious Diseases, University of Rome Tor Vergata, Rome, Italy

Our previous studies have reported an association between cellassociated HIV-1 DNA levels, plasma HIV-1 RNA load and CD4+ T cell counts in highly active antiretroviral naive HIV-1 patients. In the framework of a study aimed at monitoring markers of AIDS progression, we evaluated HIV-DNA levels in peripheral blood mononuclear cells (PBMCs) collected from long-term HAART- treated HIV-1 infected patients. HIV-DNA levels were quantified in serial PBMCs samples from $47 \mathrm{HIV}-1$ infected patients before and after $8,13,21,27,35,42,47,59$ months of HAART, using quantitative real-time PCR. Also, we compared changes of cellular HIVDNA level with concomitant variations in plasma viral load and CD4+ T cell counts. Results showed that after 5 year of antiretroviral therapy, cellular HIV DNA declined significantly by a median of tenfold. Furthermore we found that plasma viremia decreased along with the decay of cell-associated HIV-1 DNA levels during antiviral treatment. As expected an inverse correlation between HIV-DNA levels in PBMCs and CD4+ T cell counts was found. Interestingly cellular HIV DNA levels measured in HIV-1 infected patients during antiviral therapy were influenced by pre-HAART levels of HIV-DNA in PBMCs. Specifically a lower baseline levels of HIV-DNA correlates with lower levels of HIV-DNA during HAART therapy (and viceversa). It should be also noted that some patients experienced a reduction in HIV-DNA to undetectable levels while others had detectable HIV-DNA levels even in presence of plasma viremia $<50$ copies $/ \mathrm{ml}$. This observation suggests that low levels of viral replication and/or residual cellular reservoir might persist also during an effective HAART treatment in different extent between patients. All together these findings reinforce the importance of HIV-DNA evaluation, in addition to HIV-RNA and CD4+ T cell counts, in physiopathological and therapeutical studies, particularly in an era of research aimed at diminishing the HIV reservoir. Even if blood represents a small part of this reservoir, our study suggests that HIV-DNA in blood is a reliable marker that provides useful informations on the effectiveness of treatment and on entity of cellular reservoir.

\section{CLINICAL SCIENCES}

\section{PO 13}

EVALUATION OF A NEW REAL-TIME PCR METHOD FOR HIV-1 RNA QUANTIFICATION IN PATIENTS WITH LOW LEVEL OF VIREMIA

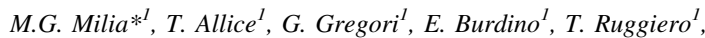

B. Simoncelli ${ }^{1}$, A. Bottoni ${ }^{1}$, C. Galli ${ }^{2}$, S. Bonora ${ }^{3}$, G. Orofino ${ }^{3}$, G. Di Perri ${ }^{3}$, V. Ghisetti ${ }^{1}$

${ }^{1}$ Laboratory of Microbiology and Virology, Amedeo di Savoia Hospital, Turin, Italy, ${ }^{2}$ Scientific Affairs, Abbott Diagnostic, Rome, Italy; ${ }^{3}$ Department of Infectious Diseases, Amedeo di Savoia Hospital and University of Torino, Turin, Italy

Objectives: To assess performances of the recently introduced Cobas Ampliprep/Cobas TaqMan HIV-1 version 2 (CAP/CTM-2) with a detection limit of 20 copies/ml compared with the Cobas Ampliprep/ Cobas TaqMan HIV-1 version 1 (CAP/CTM-1, lower detection limit 40 copies $/ \mathrm{ml}$ ), in plasma specimens from HIV-1 infected individuals that experienced undetectable or low but detectable viremia.

Methods: Two-hundred samples consisting of two sets were selected and tested with the two PCR techniques. The first set $(\mathrm{n}=122)$ included samples with HIV-1 RNA undetectable or $<40$ copies/ml measured with CAP/CTM-1 and the second one ( $\mathrm{n}=78$ ) of samples with HIV-1 RNA between 20 and 40 copies $/ \mathrm{ml}$ with CAP/CTM-2. Replicates of a serially diluted HIV-1 RNA standard (Acrometrix, Benecia, US) from 107 to 2 copies $/ \mathrm{ml}$ has been used to evaluate the sensitivity of CAP/CTM-2. Test reproducibility has been assessed on replicates of 146 CAP/CTM-2 positive samples with HIV-1 RNA between 21 and 200 copies $/ \mathrm{ml}$. In those patients reporting an undetectable HIV-1 RNA or $<40$ copies/ml with CAP/CTM-1 and a positive results with CAP/ CTM-2, viral evolution over time was evaluated with a longitudinal analysis covering one-year follow-up after the undetectable measurement with CAP/CTM-1. 
Results: Concordance between the two tests was 66\% (132/200 concordant samples) with 68 discordant samples; in 55 samples HIV1 RNA was undetectable (target not detected, TND) with CAP/CTM1 but detectable with CAP/CTM-2 (21-304 copies/ml); in 13 samples HIV-1 RNA was $<40$ copies/ml with CAP/CTM-1 but $>40$ copies $/ \mathrm{ml}$ with CAP/CTM-2 (44-831 copies/ml). As overall, HIV-1 RNA was undetectable (TND) with CAP/CTM-1 and CAP/CTM-2 in 111 $(55.5 \%)$ and $50(25 \%)$ specimens, respectively and detectable in 89 $(44.5,12.5 \%$ of them within the test linear range) and $150(75,62 \%$ of them within the test linear range) samples with CAP/CTM-1 and CAP/CTM-2, respectively. Serial dilutions of known HIV-1 RNA standard showed that the detection rate of CAP/CTM at 100 copies/ $\mathrm{ml}$ is $100 \%$ while between 20 and 50 is $50 \%$. Assay for CAP/CTM-2 reproducibility showed a 35\% CV\% for HIV-1 RNA levels from 21 to 200 copies $/ \mathrm{ml}$, with a detection rate on repeated testing of $71 \%$. Viral evolution with CAP/CTM-2 over one-year of follow-up in 85 patients (340 plasma specimens) with undetectable HIV-1 RNA at the first sample with CAP/CTM-1, showed that HIV-1 RNA was consistently detectable in 45 patients $(53 \%)$ but in only a minority of them (n: 8 , $26 \%$ ) HIV-1 RNA was $>200$ copies/ml at least in one sample with mutations conferring HIV-1 drug resistance in $1 / 8$ patients; while in 40 patients (47\%) HIV-1RNA was persistently undetectable over time also with CAP/CTM-2.

Conclusion: Lowering the detection limit of real-time PCR assay for HIV-1 RNA quantification leads to the persistent detection of low level of HIV-1 RNA in many patients forcing a reconsideration of viremia levels for defining the virologic success of antiretroviral therapy.

\section{PO 14 \\ CARDIOVASCULAR RISK FACTORS IN PATIENTS ON LONG- TERM TREATMENT

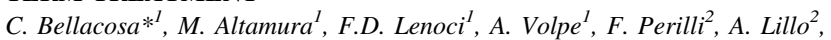 G. Angarano ${ }^{I}$, P. Maggi ${ }^{l}$ \\ ${ }^{1}$ Malattie Infettive, Azienda Ospedaliera Policlinico, Bari, Italy, ${ }^{2}$ Chirurgia \\ Vascolare, Azienda Ospedaliera Policlinico, Bari, Italy}

Objectives: The aim of this study was to evaluate the cardiovascular risk among patients treated for more than 5 years with regimens based on nevirapine or efavirenz.

Patients and methods: A total of 276 patients were retrospectively evaluated, 156 of whom were treated with nevirapine and 120 with efavirenz, by examining traditional risk factors and detecting the presence of subclinical carotid lesions with colour-Doppler ultrasonography.

Results: When comparing the data at baseline and follow-up in the nevirapine group, total cholesterol, low-density lipoprotein cholesterol (LDLc) and triglycerides showed a significant decrease, while high-density lipoprotein cholesterol increased. Ultrasound data, obtained in a subgroup of 67 patients, did not show significant changes for those treated with nevirapine. In the efavirenz group, total cholesterol, LDLc, triglycerides, glycaemia, body mass index and the number of patients with a pathological ultrasound significantly increased. When comparing the two groups at baseline and follow-up, nevirapine patients had significantly higher values of total cholesterol, LDLc and triglycerides at baseline, while total cholesterol and LDLc differed non-significantly at follow-up; triglycerides became significantly lower in the nevirapine arm with respect to the efavirenz group. Glycaemia was comparable between the two groups at baseline, while it was significantly lower in the nevirapine group at follow-up. The number of pathological ultrasound findings was significantly higher in the efavirenz group at follow-up.

Conclusions: Patients treated with nevirapine demonstrated a better lipid and glucose profile and a lower tendency to develop subclinical atherosclerotic lesions.

\section{PO 15}

HLA-B*5701 TYPING BY ALLELE SPECIFIC REAL-TIME PCR: COMPARISON WITH SEQUENCE BASED TYPING

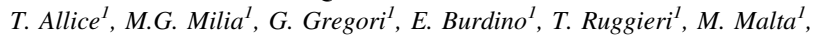
E. Scuccimarra ${ }^{I}$, V. Ghisetti ${ }^{1}$

${ }^{I}$ Laboratory of Microbiology and Virology, Amedeo di Savoia Hospital, Turin, Italy

Background: Hypersensitivity reaction to abacavir is strongly associated to the presence of the HLA-B*5701 allele. The identification of susceptible individuals prior to the institution of abacavir therapy is therefore of clinical importance and has generated the demand for a simple and rapid test to identify the HLA-B*5701 allele.

Aim: In this work we compared a method based on allele-specific real-time PCR and melting curve analysis (AS-PCR) with the direct sequencing based typing (SBT), that is the gold standard method for identifying HLA-B*5701 allele.

Methods: Genomic DNA was extracted by whole blood using the NucliSENS easyMAG (NeM, BioMerieux, France) a semi-automated platform with an extraction protocol based on magnetic-silica particles, according to the manufacturer's instruction. To identify the HLA-B*5701 allele we used a commercial test based on allele-specific polymerase chain reaction and melting curve analysis (AS-PCR, Duplica Real Time HLA-B*5701 Genotyping Kit, Euroclone, Italy) compared with the direct sequencing kit HLA*B5701 Sequence Based Typing (SBT, Abbott, Italy) as gold standard.

Results: We analysed 46 patient samples with SBT: 9 resulted HLA$\mathrm{B} * 5701$-positive while 37 were HLA-B*5701-negative samples that included closely related HLA-B*5701 alleles $-\mathrm{B} * 5702(\mathrm{n}=1)$, $B * 5703(n=4),-B * 5301(n=3)$ and $B * 5801(n=3)$. With ASPCR, we obtained the same results of SBT test: 9 samples HLA$B * 5701$-positive and 37 HLA-B*5701-negative. All 11 closely related HLA-B*5701 samples were identified as HLA-B*5701-negative. Compared with SBT, this method had $100 \%$ sensitivity and specificity for the HLA-B*5701 allele.

Conclusion: The method based on allele-specific real-time PCR positively identified all HLA-B*5701 alleles in concordance with their SBT-assigned typing and was able to distinguish between HLA$\mathrm{B} * 5701$, closely related HLA-B*57 alleles and non-HLA-B $* 57$ alleles and gave results in one working-day. In conclusion, this method is a rapid, sensitive, specific and may be suitable for large or small scale screening for the HLA-B*5701 allele.

\section{PO 16}

IDENTIFICATION OF HLAB 5701 IN GENOMIC DNA EXTRACTED FROM PERIPHERAL BLOOD OF HIV-1 POSITIVE PATIENTS

N. Cuomo ${ }^{1}$, A. Di Spirito, , G. Milone ${ }^{I}$, N. Melillo ${ }^{1}$, M. Dati ${ }^{1}$, C.Esposito* ${ }^{\prime}$

${ }^{1}$ UOC Virologia, Naples, Italy

Background: The work describes the association between the HLAB5701 and the hypersensitivity to Abacavir in patients receiving antiretroviral therapy. The HLAB57 family consists of at least 16 related genes and gene products 5701 were associated with hypersensitivity to Abacavir. For these reasons, in March 2008 was reached an agreement between the ERA and AIFA on a screening to detect the HLAB5701 before starting Abacavir treatment in the patients with HIV1. At the end of this study the presence of the allele was associated with a lower viral load in some patients HIV1 positive. The clinical validity of this screening is, therefore, in the identification of HIV patients potentially subjected to hypersensitivity reaction to Abacavir.

Methods: The aim of the work was to verify the HLAB5701 in African and European patients, using a genetic test. The method used is a real-time PCR for HLAB5701. The test is based on two major processes: isolation of genomic DNA from specimens and real-time amplification with allele specific primers. The real-time PCR 
monitoring of fluorescence intensities allows the accumulation of the detection product without reopening of reaction tubes after the PCR run. HLAB5701 RealTM PCR kit is a qualitative test; it contains the Internal Control IC (beta-globine gene), which allows the control the presence of cellular material in the sample.

Results: 44 patients, also from continents other than Europe, including 34 males and 10 females, aged between 20 and 64 years were subjected to screening for the determination of the HLAB5701. Only 4 patients, of Caucasian origin, were positive; of the 40 negative people only 12 were from European (27.3\%), confirming that this allele in Africa occurs with a frequency much lower than in the European continent. From the positive cases in only one patient was found the allele associated with low viral load. This could be a rare case of long-term non progressor, or of a patient in which the virus has a high replicative activity. In such case, the presence of the allele should not be considered a negative factor, correlated to a possible treatment failure but rather represents a case of slow progression of the infection, which can be maintained for decades.

Conclusions: The correlation between HLAB5701 and hypersensitivity to abacavir leads to the conclusion that this reaction is certainly due to a genetic component, not attributable, however, to the presence of one allele, but probably endogenous and/or environmental factor still to be clarified. Therefore, the screening of HLAB5701 is crucial for the patients not yet receiving antiretroviral therapy with Trizivir. Moreover a positive result to this test is necessary but not sufficient to establish the development of the Hypersensitivity Abacavir, but only to the increased susceptibility to this reaction. Therefore the test is predictive but not diagnostic for the development of a hypersensitivity reaction to abacavir.

\section{PO 17}

\section{BONE MINERAL STATUS IN HIV INFECTED PATIENTS IN} MEDITERRANEAN REGION: PRELIMINARY DATA

P. Di Carlo ${ }^{1}$, R. La Sala* ${ }^{1}$, D. Bruno ${ }^{2}$, P. Colletti ${ }^{1}$, G. Mazzola ${ }^{1}$, M. Mineo ${ }^{1}$, M.A. Raimondo ${ }^{1}$, G.B. Rini ${ }^{2}$, L. Titone ${ }^{I}$, G. Caruso ${ }^{3}$

${ }^{1}$ Policlinico Universitario "Paolo Giaccone, Palermo, Italy; ${ }^{2}$ Department of Clinical Medicine and Emerging Pathologies, University of Palermo, Palermo, Italy; ${ }^{3}$ Dipartimento di Biotecnologie mediche e Medicina legale, Sezione di scienze radiologiche, Università degli Studi di Palermo, Palermo, Italy

Background: The role of HIV infection in bone mineral is incompletely characterized, and studies are needed to identify the underlying mechanisms. Vitamin D deficiency is highly prevalent in HIV individuals both before than after Antiretroviral Therapy (ARV). Recent studies conducted in sunny countries have observed a high prevalence of vitamin D deficiency.

Objectives: Our region is one of sunny countries and here, we investigated the bone mineral status in HIV population and control. Material and methods: The clinical data associated with bone loss from 2010 to 2011 were analyzed, including 35 patients treated with HAART (named treated group; mean age $45.5 \pm 4$, range 38-65), 10 HIV-infected antiretroviral-naive patients (named untreated group; mean age $35.5 \pm 12$, range 20-45) and 20 healthy people (named control group; mean age $48 \pm 6$, range 40-68). All participants had measurements of bone mineral density (BMD) and bone-related laboratory parameters.

Results: Osteopenia and osteoporosis were diagnosed in 6 out 35 $(17 \%)$ and 10 out $35(30 \%)$, respectively, of treated group and 10 out $20(50 \%)$ and 3 out $20(15 \%)$, respectively of control group. All patients of untreated group have normal weight-adjusted bone mineral density (BMD). Vitamin D deficiency were observed in a HIV treated patient and in 2 control patients. Low levels of plasma phosphorus were detected in 9 out $35(26 \%)$ treated patients.

Discussion: In our data, Osteoporosis was more frequently observed in HIV treated group. Among biochemical markers, plasma phosphorus test was indicator of BMD loss. On the contrary to other studies conducted in sunny countries, no vitamin $\mathrm{D}$ deficiency was observed.

\section{PO 18}

IMPACT OF AGING AND HIV-INFECTION ON NEUROCOGNITIVE PERFORMANCE

N. Ciccarelli ${ }^{\prime}$, M. Fabbiani ${ }^{I}$, E. Baldonero ${ }^{I}$, I. Fanti ${ }^{I}$, S. Farina ${ }^{l}$,

A. D'Avino ${ }^{1}$, A. Mondi', L. Sidella', M. Doino ${ }^{I}$, M.C. Silveri ${ }^{I}$,

S. Di Giambenedetto ${ }^{I}$

${ }^{I}$ Catholic University, Rome

Background: Despite the availability of Highly Active Antiretroviral Therapy (HAART), minor cognitive disorders are increasingly recognized in HIV-infected patients. The longer duration of HIV disease, as a consequence of HAART, together with an older age at seroconversion, may increase the risk for cognitive impairment. Aim of the study was to explore the interactions between aging and HIV infection in the expression of cognitive decay.

Methods: A cross-sectional single cohort study was performed, consecutively enrolling asymptomatic HIV+ subjects during routine outpatient visits. All patients underwent an extensive Neuropsychological Battery. A control population of $36 \mathrm{HIV}$-negative individuals matched for age and education was also selected. Comparisons between groups were based on Mann-Whitney $U$ test; to control for the probability of committing a type I error in multiple comparisons, the Bonferroni correction was adopted by setting the $\mathrm{p}$ value at 0.003 . The impact of aging and HIV infection on neuropsychological performance was investigated by factorial analysis of variance.

Results: One hundred fifty-three patients [59.5\% males, median age 47 (IQR 41-56) years, $13.1 \%$ older than 65 years, median education 12.0 (IQR 8-14.5) years, 25\% with past AIDS-defining events (non neurological), $77.8 \%$ with HIV-RNA $<50$ copies $/ \mathrm{mL}$, median CD4 cells count 518 (IQR $380-703), 88.2 \%$ on HAART] were enrolled. Aging $(\geq 65$ years $)(p=0.0008)$ and HIV-positive status $(p=0.009)$ showed a significant effect on the total number of pathological tests. However, no significant interaction between aging and HIV was observed. These effects were confirmed exploring any single task. Moreover, comparing younger $\mathrm{HIV}+$ patients (age $<65 ; \mathrm{N}=133$ ) with older control subjects (age $\geq 5$ years, $\mathrm{N}=20$ ), no significant difference was found in the neuropsychological performance obtained at each test, with the exception of better scores obtained by younger HIV+ patients on the Trail Making Test B (time) $(\mathrm{p}=0.001)$ and Stroop Test (time) $(\mathrm{p}=0.001)$.

Discussion: Data available in the literature about the interaction of aging and HIV on cognitive status are not uniform. In our study HIV+ patients obtained lower neuropsychological performance than matched control population. At the same time, aging showed a negative impact on cognitive performance, but no direct evidence of interaction between aging and HIV was observed. However, the cognitive performance of younger HIV+ patients and older negative adults was qualitatively and quantitatively comparable, except for performances in tasks exploring processing speed, supporting the "cognitive slowing hypothesis" for older people. In conclusion, HIV disease enhances the risk of cognitive decay in young subjects. Our results suggest that aging and HIV concur to produce the cognitive decay. Further analyses are need to better understand the interplay of factors in the expression of neurodegeneration in HIV infection.

\section{PO 19}

AIDS DEMENTIA COMPLEX IN THE HAART ERA:

CHARACTERIZATION AT DIAGNOSIS, MORTALITY AND PREDICTORS OF DEATH AT THE DEPARTMENT OF INFECTIOUS DISEASES IN BRESCIA (NORTHERN ITALY)

A. Bonito $*^{I}$, M. Mendeni ${ }^{I}$, D. Motta ${ }^{I}$, E. Focä ${ }^{I}$, I. Izzo ${ }^{I}$, S. Casari ${ }^{I}, F$. Castelnuovo $^{I}$, A. Scalzini ${ }^{I}$, G. Paraninfo ${ }^{I}$, G. Carosi ${ }^{I}$, F. Buelli ${ }^{I}$, M. Magoni $^{2}$, E. Quiros-Roldan ${ }^{I}$, C. Torti ${ }^{1}$

${ }^{2}$ Institute of Hygiene, University of Brescia, Brescia, Italy; ${ }^{1}$ Infectious Diseases Dpt, University and Spedali Civili of Brescia, Brescia, Italy 
Background: AIDS dementia complex (ADC) account for about $6 \%$ of the AIDS defining diagnoses over the last decade in Italy. Patients' characteristics at the time of ADC in terms of exposure to combination antiretroviral treatment (cART), clinical and immunevirological status, time elapsed from HIV diagnosis, and outcome have been poorly investigated.

Methods: We revised patients' files of ADC cases from Jan 1996 to Dec 2010. Descriptive analysis was performed at ADC diagnosis. Kaplan-Meier analysis assessed mortality and Cox models was used to investigate possible predictors of death.

Results: Diagnosis of ADC was made in 141 patients (mean age: 40.2 [SD 9.2] years; $76 \%$ males; $64 \%$ intravenous drug use as risk factor for HIV) after a median of 7.5 years since HIV diagnosis, $23 \%$ patients having ADC $<1$ year after HIV diagnosis ( $10 \%$ not later than 1 month). CD4+ count was lower in patients who had $\mathrm{ADC}=12$ months before HIV diagnosis than $>12$ months. However, CD4+ count was low in both groups (mean $123 / \mathrm{mm}^{3}$, SD 134 vs. $200 / \mathrm{mm}^{3}$, SD $198 / \mathrm{mm}^{3}$; $\mathrm{p}=0.04$ ). Moreover, only in patients who had HIV diagnosis more than 10 years before ADC the rate of cART prescription was higher ( 49 vs. $31 \% ; \mathrm{p}=0.046$ ). There were 60 deaths (42\% patients) over 799 patient-years of follow-up (PYFU) accounting for a incidence of $7.5 \%$ per PYFU (95\% CI 5.8-9.7\%) with $25 \%$ deaths occurring in the first 2 years with a median survival time of 11.3 years. Causes of death were: major opportunistic infections (including ADC) in 45/60 (75\%) patients, non-AIDSdefining events in 14/60 (23\%), and suicide in 1 patient. At multivariable analyses, death was associated with: CD4+ T-cell count below 100 (HR 2.64, 95\% CI 1.41-4.94; $\mathrm{p}=0.002$ ), not receiving any cART (HR: $5.39,95 \%$ CI 2.26-12.9; $<<0.0001$ ), and age at the time of ADC (HR: 1.03 for each year increase; $p=0.037$ ).

Conclusions: A large percentage of patients had ADC after being seen for long time without cART. This may indicate the need of accurate screening protocols for early diagnosis of neurocognitive impairment that may lead to ADC and cART initiation according with current Italian guidelines. Likewise, low CD4+ count and absence of cART had a significant impact on long term mortality after ADC.

\section{PO 20}

ALEXITHYMIA, A DISORDER OF THE REGULATORY MECHANISM OF EMOTION ELABORATION, AS A PREDICTOR OF NEUROCOGNITIVE IMPAIRMENT IN HIV INFECTED PATIENTS

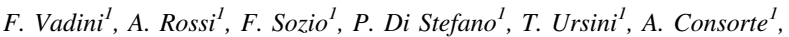
G. Parruti $^{\text {I }}$

${ }^{I}$ U.O. Malattie Infettive, Pescara, Italy

Alexithymia, an impairment of affective and cognitive emotional processing, is often associated with human immunodeficiency virus (HIV) and may reflect effects of the virus on several brain areas. We hypothesized that there would be a correlation between extent of alexithymia and cognitive performance including attention, executive functions, speed of information processing, verbal fluency, memory, and verbal learning. HIV infected patients were consecutively enrolled from November, 2010 to January, 2011. We investigated a several psychological factors, including Alexithymia, using the 20-item Toronto-Alexithymia-Scale (TAS-20, cut-off $=61$ ), Type-D personality using the DS14 Distress Scale (Negative Affectivity Scale, cut-off $=9$; Social Inhibition Scale, cut-off $=9$ ), Depression symptoms, using the Beck Depression Inventory-II (BDI-II, cutoff $=15$ ) and QoL, with PCS (Physical Component Summary) and MCS (Mental Component Summary) using the SF12 questionnaire. Screening of cognitive function was carried out with classical neuropsychological tests including Trail Making Test A (TMT-A), Trail Making Test B (TMT-B), Digit Span Test (DST), Digit Symbol Modalities Test (DSMT), Auditory Verbal Learning Test (AVLT), Verbal Fluency "S" (VF-S). All statistical analyses were carried out using the Stata 10.0 package. We enrolled 33 patients, $60.61 \%$ males, aged $44.03 \pm 8.2$. Alexithymia was diagnosed in $15,2 \%$ of patients, TypeD personality in $45.5 \%$, depression symptoms in $36.4 \%$ of patients; mean PCS and MCS were $47.3 \pm 8.3$ and $39.6 \pm 12.4$. Performance at neuropsychological tests was clear cut ( $>2$ DS) altered in: TMT-A: 5 patients (15.2\%); TMT-B: 5 patients (15.2\%); DST 14 (42.4\%); DSMT: 16 (48.5\%); AVLT: 4 (12.1\%); VF-S: 3 (9\%). At least one significant deficit was identified in $27(81.8 \%)$ of patients; deficits in at least 2 cognitive domains in $20(60.6 \%)$ patients. An Alexithymia score $<61$ had a very high negative predictive value for neurocognitive impairment: $83.3 \%$ (5/6 patients) for no deficit; $90 \%$ (18/20 patients) for $=2$ domains involved. If our observation will be confirmed on a larger series, performing a personality assessment in HIV infected patients and, in particular, measuring Alexithymia with TAS-20, may prove a simple and effective way to rule out clinically relevant neurocognitive impairment.

\section{PO 21}

MINIMAL EMERGENCE OF DARUNAVIR RESISTANCE AT TREATMENT FAILURE: ARE INTERPRETATION ALGORITHMS ADEQUATE?

G. Sterrantino*I, M. Zaccarelli ${ }^{2}$, M. Zazzi ${ }^{3}$, A. De Luca ${ }^{4}$, V. Borghi ${ }^{5}$,

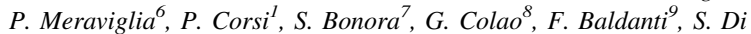

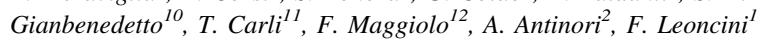
${ }^{I}$ Malattie Infettive, Azienda Ospedaliera Universitaria Careggi, Florence, Italy; ${ }^{2}$ Istituto Nazionale per le Malattie Infettive "Lazzaro Spallanzani", Rome, Italy; ${ }^{3}$ Università di Siena, Dipartimento di Biologia Molecolare, Siena, Italy; ${ }^{4}$ Istituto di Clinica Malattie Infettive, Rome, Italy; ${ }^{5}$ Clinica Malattie Infettive, Modena; ${ }^{6}$ Seconda Divisione Malattie Infettive Ospedale L. Sacco, Milan, Italy; ${ }^{7}$ Malattie Infettive Amedeo di Savoia, Turin, Italy; ${ }^{8}$ Virologia Careggi, Florence, Italy; ${ }^{9}$ Virologia S. Matteo, Pavia, Italy; ${ }^{10}$ Laboratorio Virologia Cattolica, Rome, Italy; ${ }^{11}$ Malattie Infettive, Grosseto, Italy;

${ }^{12}$ Malattie Infettive, Bergamo, Italy

Background: Due to the high genetic barrier of Ritonavir-boosted Darunavir (DRV), resistance to this drug is associated with multiple mutational profiles. We used a large database of HIV patient, who were treated with a DRV-containing regimen after treatment failure to investigate protease (PR) evolution under DRV therapy.

Methods: Data were collected from patients included in the Italian ARCA database with a genotypic resistance test (GRT) done within 3 months before DRV start. The protease mutational pattern before and after DRV failure was analysed according to IAS-USA criteria. Results: A total of 840 pre-treated patients were included: 759 (89\%) patients had still undetectable viral load after a median of 54 weeks (IQR 23-112) of DRV-containing regimen and 81 (9.5\%) experienced virological failure (median 43 weeks, IQR 18-62). Baseline GRT of the 81 failing patients had a mean of $3.2 \pm 2.0$ major PR mutations as compared to $2.5 \pm 1.9$ in patients without failure. A significantly higher proportion of specific PR mutations was detected, including V32I $(21.0$ vs. $7.6 \%, p=0.0048) ; \quad$ I47V $(24.7$ vs. $10.3 \%$, $\mathrm{p}=0.0003) ; \mathrm{I} 54 \mathrm{M}(12.3$ vs. $4.3 \%, \mathrm{p}=0.0043)$; I84V (40.7 vs. $27.5 \%, \mathrm{p}=0.0018$ ). Moreover, in the 81 failing patients, comparing baseline and failure GRT, the following mutations increased in frequency: V32I (40.7\% at failure vs. $21.0 \%$ at baseline); I50V (12.3 vs. 4.9\%); I54L (27.9 vs. $7.4 \%)$; Q58E (22.2 vs. $14.8 \%)$ and T74P (7.4 vs. $3.7 \%$ ). The mean number of mutations per patient increased from 3.2 at baseline to $3.6( \pm 2.2)$ at DRV-failure. Using the Stanford interpretation algorithm, at baseline, none of the failing patients harboured a virus that was fully resistant to DRV, 39 (48.1\%) had intermediate resistance and $45(55.6 \%)$ were fully susceptible, 18 of which $(22.2 \%)$ having no PR resistance mutation. At DRV failure, only 2 patients $(2.5 \%)$ had developed full resistance to the drug, 45 $(55.6 \%)$ had intermediate resistance and $33(40.7 \%)$ were still susceptible to DRV, 15 (18.5\%) patients without any PR mutation.

Conclusions: In this cohort analysis, protease mutations associated with reduced virological response to DRV were in agreement with 
currently used algorithms. However, susceptibility to DRV was generally maintained even in the presence of virological failure of regimens containing the drug. Indeed, for the Stanford interpretation algorithm, full resistance to DRV is uncommon, so that most patients retained partial or complete DRV susceptibility with several patients lacking any protease mutation.

\section{PO 22}

ANALYSIS OF MAJOR AND MINOR IAS-USA PI MUTATIONS IN THE MONET TRIAL OF DARUNAVIR/RITONAVIR MONOTHERAPY VERSUS DRV/R + 2NRTIS

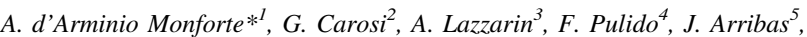
A. Hill ${ }^{6}$, Y. van Delft ${ }^{7}$, C. Moecklinghoff

${ }^{1}$ San Paolo Hospital, Milan, Italy, Milano; ${ }^{2}$ University of Brescia, Brescia, Italy; ${ }^{3}$ S. Raffaele Hospital, University of Milan, Milan, Italy; ${ }^{4}$ Hospital 12 de Octubre, Madrid, Spain; ${ }^{5}$ Hospital la Paz, Madrid, Spain; ${ }^{6}$ Liverpool

University and Tibotec BVBA, Liverpool, UK; ${ }^{7}$ Janssen-Cilag, Tilburg, The Netherlands; ${ }^{8}$ Anssen-Cilag, Neuss, Germany,

Background: For patients on treatment with HIV RNA $<50$ copies/ $\mathrm{mL}$, it is unknown whether the genetic barrier to evolution of resistance is different for DRV/r monotherapy, compared with standard triple combinations of antiretrovirals. Several minor IAS-USA mutations are detected frequently in samples from PI naïve patients. Methods: In the MONET trial, 256 patients with no history of virological failure and HIV RNA $<50$ copies/mL on current HAART (NNRTI based $(43 \%)$, or PI based $(57 \%)$ switched to either DRV/r monotherapy $(800 / 100 \mathrm{mg}$ OD) versus DRV/r + 2NRTIs. HIV RNA levels were evaluated at Weeks 2, 12, 24, 36, 48, 60, 72, 84 and 96: all patient samples with HIV RNA above 50 copies/mL were sent for genotypic resistance analysis (VircoTYPE HIV-1, Beerse, Belgium). Virtual phenotype was also assessed when PI mutations were detected. The percentage of patients with major or minor IAS-USA PI mutations was analysed by treatment arm.

Results: Patients were $81 \%$ male and $91 \%$ Caucasian, with median age 43 years, and median CD4 count of 575 cells $/ \mu \mathrm{L}$. While patients were receiving randomised treatment, HIV RNA was above 50 copies $/ \mathrm{mL}$ for 47/1,051 (4.5\%) patient-visits in the DRV/r + 2NRTI arm and 69/1,009 (6.8\%) patient-visits in the DRV/r monotherapy arm. Of 48 patients with at least one successful genotype (27 in the DRV/r monotherapy arm, 21 in the DRV/r + 2NRTI arm), two showed major IAS-USA PI mutations during short-term elevations in HIV RNA (one per treatment arm). Both patients remained phenotypically sensitive to darunavir, with sustained HIV RNA $<50$ copies/mL during the trial and no change in antiretroviral treatment. The five most common minor IAS-USA mutations detected in the DRV/r mono and DRV/r + 2NRTI arms were L63P $(78,62 \%$ respectively), I93L (59\%, 19\%), V77I (33, 43\%), I62V (22, 33\%) and I64V $(15,24 \%)$. These five mutations were also commonly observed in the Stanford HIV database of 7,601 samples from PI naive patients. Fourteen patients in the DRV/r monotherapy arm had repeated genotypes during intermittent low-level viraemia - there was no evidence for evolution of minor IAS-USA PI mutations over time in these patients.

Conclusions: After 96 weeks of treatment in the MONET trial, there is no evidence for an increased risk of emergence of major or minor IAS-USA PI mutations with DRV/r monotherapy, compared to DRV/r + 2 NRTIs, and no evidence for evolution of PI mutations after repeated genotyping.

\section{PO 25}

PHARMACOKINETIC (PK) AND PHARMACODYNAMIC ANALYSES OF ONCE- AND TWICE-DAILY DARUNAVIR/RITONAVIR (DRV/r) IN THE ODIN TRIAL

A. Lazzarin $*^{1}$, F. Mazzotta ${ }^{2}$, I. Mezzaroma ${ }^{3}$, V. Sekar ${ }^{4}$, G. De La Rosa ${ }^{4}$,

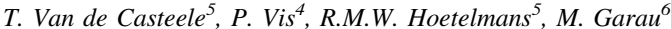

${ }^{1}$ S. Raffaele Hospital, University of Milan, Milan, Italy; ${ }^{2}$ S.M. annunziata Hospital, Firenze, Florence, Italy; ${ }^{3}$ Department of Clinical Medicine, Sapienza, University of Roma, Rome, Italy; ${ }^{4}$ Tibotec Inc, Titusville, NJ, USA;

${ }^{5}$ Tibotec BVBA, Beerse, Belgium; ${ }^{6}$ Medical Affairs Janssen, Milan, Italy
Background: In the Phase III, randomised, open-label ODIN trial, treatment-experienced HIV-1-infected adults with no screening DRV resistance-associated mutations received DRV/r $800 / 100 \mathrm{mg}$ qd or DRV/r 600/100 mg bid (both arms $+=2$ NRTIs). At Week 48, 72.1\% qd versus $70.9 \%$ bid patients achieved HIV-1 RNA $<50$ copies $/ \mathrm{mL}$ (95\% CI -6.1 to $8.5 \%, p<0.001$; ITT-TLOVR), confirming non inferiority of DRV/r qd. The relationship between DRV PK and efficacy and safety following treatment with DRV/r is explored.

Methods: Sparse blood sampling for PK evaluations was taken at Weeks 4, 8, 24 and 48 to determine DRV trough concentrations (COh) and exposure (AUC24h, calculated as AUC12h $\times 2$ for bid) using a population pharmacokinetic model. Relationships between PK parameters and efficacy [change in $\log _{10}$ HIV-1 RNA and virological response (HIV-1 RNA <50 copies/mL)] were assessed using ANCOVAs. Relationships between PK parameters and occurrence of adverse events of interest and laboratory lipid abnormalities were evaluated using descriptive statistics.

Results: PK data were available for $280 \mathrm{DRV} / \mathrm{r}$ qd patients and 278 bid patients. Median (range) COh was 1,896 (184-7,881) ng/mL for DRV/r qd and 3,197 (250-11,865) ng/mL for DRV/r bid. Median (range) AUC24h for DRV/r qd was 87,788 $(45,456-236,920) \mathrm{ng} \mathrm{h} /$ $\mathrm{mL}$ and $109,401(48,934-323,820) \mathrm{ng} \mathrm{h} / \mathrm{mL}$ for bid. No relevant relationships were observed between DRV PK and efficacy: changes from baseline in HIV-1 RNA $\log _{10}$ copies $/ \mathrm{mL}$ at Week 48 for pooled data by DRV AUC24 h quartile ranges $(=79,576 ; 79,577-100,376$; 100,377-119,356; >119,356 ng h/mL) were $-2.06,-2.22,-2.19$, and $-2.08 \log _{10}$ copies $/ \mathrm{mL}$, respectively. The $\%$ patients achieving HIV-1 RNA $<50$ copies/mL by these quartile ranges were $82.0,88.5$, 82.6 and $76.5 \%$ (observed data), respectively. In a logistic regression analysis adjusting for baseline viral load, AAG levels and number of sensitive NRTIs in the optimized background regimen, there were no relevant relationships between PK and virological response. No apparent relationships were observed between DRV PK and occurrence of rash-, cardiac-, GI-, liver-, lipid- and glucose-related AEs or laboratory lipid abnormalities.

Conclusions: Dosing with DRV/r 800/100 mg qd resulted in lower COh and AUC24h compared to DRV/r 600/100 mg bid; however, comparable efficacy between DRV/r qd and bid confirmed adequate DRV concentrations were achieved following qd dosing. No relevant relationships were observed between DRV PK and efficacy or safety at Week 48.

\section{PO 26}

HEALTH-RELATED QUALITY OF LIFE (HRQOL) ASSESSMENT WITH ONCE- AND TWICE-DAILY DARUNAVIR/RITONAVIR (DRV/ R) IN THE ODIN TRIAL

A. Lazzarin*1, F. Mazzotta ${ }^{2}$, I. Mezzaroma ${ }^{3}$, T. Van de Casteele 4 , G. De La Rosa ${ }^{5}$, P. De Doncker ${ }^{4}$, R. Termini ${ }^{6}$

${ }^{1}$ S.Raffaele Hospital, University of Milan, Italy, Milan; ${ }^{2}$ S.M. annunziata Hospital, Firenze, Florence, Italy; ${ }^{3}$ Department of Clinical Medicine, Sapienza, University of Roma, Rome, Italy; ${ }^{4}$ Tibotec BVBA, Beerse, Belgium, Beerse; ${ }^{5}$ Tibotec Inc, Titusville, USA; ${ }^{6}$ Medical Affairs Janssen Italy, Milano

Background: The open-label, Phase III, ODIN trial randomised treatment-experienced HIV-1-infected patients with no DRV resistance-associated mutations (RAMs) to receive DRV/r 800/100 mg qd or DRV/r 600/100 mg bid, plus an optimized background regimen (=2 NRTIs). Non-inferiority in the primary endpoint of virological response at Week 48 was demonstrated with DRV/r qd versus bid dosing: 72.1 versus $70.9 \%$ of patients, respectively, achieved HIV-1 RNA $<50$ copies/mL (95\% CI $-6.1,8.5 ; \mathrm{p}<0.001$; ITTTLOVR). The current analysis explores patient-reported HRQoL.

Methods: Treatment-experienced patients with no DRV RAMs at screening and HIV-1 RNA $>1,000$ copies $/ \mathrm{mL}$ were randomised. Patient-reported HRQoL was measured with the Functional Assessment of HIV-infection (FAHI) questionnaire at baseline and at Weeks 
4, 12, 24 and 48 (or withdrawal visit). FAHI score at Week 48 was modeled by means of an ANCOVA, and the evolution of the FAHI score over time by means of a longitudinal mixed model, each with treatment as a factor and CD4 and baseline HIV-1 RNA as a regressor. FAHI response was defined as the proportion of patients with a clinically meaningful difference (relative increase of 10\%) in total FAHI imputed score versus baseline.

Results: HRQoL data were available for $262 / 294 \mathrm{DRV} / \mathrm{r}$ qd and 268/296 DRV/r bid patients. The baseline total FAHI imputed score was relatively high (124.1 and 121.2 for DRV/r qd and bid, respectively), leaving limited room for improvement. Mean (SE) increase in total FAHI score from baseline (ITT-LOCF) at Week 48 was comparable with DRV/r qd and bid dosing. A mean increase in total FAHI score from baseline was observed in both treatment groups at all timepoints. No relevant between-group differences were noted either by ANCOVA ( $\mathrm{p}=0.761)$ or longitudinal mixed model $(\mathrm{p}=0.995)$. There were no relevant differences between arms at any time in the proportion of FAHI responders $(\mathrm{p}=0.957)$.

Conclusions: DRV/r qd and bid dosing was comparable with respect to the increase in mean total FAHI score from baseline at Week 48 and in the proportion of patients achieving a clinically meaningful difference in total FAHI score at Week 48.

\section{PO 27}

\section{LOW-LEVEL VIRAEMIA DURING TREATMENT WITH} DARUNAVIR/R MONOTHERAPY VERSUS DRV/R + 2NRTIS IN THE MONET TRIAL

G. Carosi ${ }^{1}$, A. Lazzarin ${ }^{2}$, A. d'Arminio Monforte ${ }^{3}$, N. Clumeck ${ }^{4}$, J. Arribas $^{5}$, P. Pulick ${ }^{6}$, G. Fatkenheuer ${ }^{7}$, A. Hill ${ }^{8}$, Y. van Delft ${ }^{9}$, C. Moecklinghoff ${ }^{\prime O}$

${ }^{1}$ University of Brescia, Brescia, Italy, Brescia; ${ }^{2}$ S. Raffaele Hospital,

University of Milan, Milan, Italy; ${ }^{3}$ San Paolo Hospital, Milan, Italy; ${ }^{4} \mathrm{CHU}$ Saint-Pierre/Maladies Infectieuses, Brussels, Belgium, Brussel; ${ }^{5}$ Arribas, J., Hospital la Paz, Madrid, Spain, Madrid; ${ }^{6}$ P., Hospital for Infectious Diseases, Warsaw, Poland; ${ }^{7}$ Universitat Koln, Koln, Germany; ${ }^{8}$ Pharmacology Research Laboratories, Liverpool University, Liverpool, UK; ${ }^{9}$ Janssen-Cilag, Tilburg, The Netherlands; ${ }^{10}$ Janssen-Cilag, Neuss, Germany

Background: Patients with HIV RNA suppression below 50 copies/ mL may still have HIV RNA detectable by more sensitive PCR assay techniques.

Methods: In the MONET trial, 256 patients with HIV RNA $<50$ copies/mL on current HAART, and no history of virological failure, switched to DRV/r 800/100 mg once daily, either as monotherapy $(\mathrm{n}=127)$ or with 2NRTI $(\mathrm{n}=129)$. HIV RNA was evaluated by the Roche Amplicor Ultrasensitive assay (lower detection limit $=50$ copies $/ \mathrm{mL}$ ), for all patient visits to Week 96. With this assay, "Optical Density = background" was used to assess whether HIV RNA was detectable or undetectable below 50 copies/ $\mathrm{mL}$.

Results: Patients were $81 \%$ male, $91 \%$ Caucasian, and had median baseline CD4 count of 575 cells $/ \mu \mathrm{L}$. At the baseline visit, the percentage of patients with HIV RNA undetectable below 50 copies $/ \mathrm{mL}$ $(\mathrm{OD}=$ background $)$ was $80 \%$ in the DRV/r mono arm and $79 \%$ in the $\mathrm{DRV} / \mathrm{r}+2 \mathrm{NRTI}$ arm. The percentage with HIV RNA at different levels at the Week 96 visit is show below (observed data analysis):

\begin{tabular}{lll}
\hline HIV RNA & $\begin{array}{l}\text { DRV/r mono } \\
(\mathrm{n}=105, \%)\end{array}$ & $\begin{array}{l}\text { DRV/r }+2 \text { NRTIs } \\
(\mathrm{n}=114, \%)\end{array}$ \\
\hline HIV RNA $<50$, OD $=$ background & 79.0 & 80.7 \\
HIV RNA $<50$, detectable & 17.1 & 14.9 \\
HIV RNA 50-400 copies $/ \mathrm{mL}$ & 2.9 & 3.5 \\
HIV RNA $<400 \quad$ copies $/ \mathrm{mL}$ & 1.0 & 0.9
\end{tabular}

Including all samples from patient visits from Week 4 to Week 96, HIV RNA was above 50 copies/mL in 69/1,009 samples in the DRV/r monotherapy arm (50-400: 84\%, 400-1,000: $12 \%,>1,000: 4 \%)$ and $47 / 1,051$ samples in the DRV/r + 2NRTI arm (50-400: $83 \%$, 400-1,000: $8.5 \%,>1,000: 8.5 \%$ ).

Conclusions: In this study for patients with HIV RNA $<50$ copies/ $\mathrm{mL}$ at baseline, switching to DRV/r monotherapy showed similar levels of HIV RNA suppression to DRV/r + 2NRTIs, using more sensitive PCR assay techniques.

\section{PO 28}

CHANGES IN PATIENT-REPORTED OUTCOMES DURING THE SENSE TRIAL: FIRST-LINE TREATMENT WITH 2 NRTIS PLUS ETRAVIRINE OR EFAVIRENZ

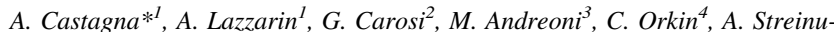

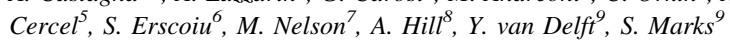

${ }^{1}$ S. Raffaele Hospital, University of Milan, Italy, Milano; ${ }^{2}$ Brescia Civili Hospital, University of Brescia, Brescia, Italy; ${ }^{3}$ Tor Vergata Hspital, University of Rome, Rome, Italy; ${ }^{4}$ St Bartholemews Hospital, London, UK; ${ }^{5}$ Clinic de Boli Infectioase si Tropicale, Bucharest, Romania; ${ }^{6}$ Clinic de Boli Infectioase si Tropicale, Bucharest, Romania; ${ }^{7}$ St Stephen's Centre, Chelsea and Westminster Hospital, London, UK; ${ }^{8}$ Liverpool University, Liverpool, UK, ${ }^{9}$ Janssen, Tilburg, The Netherlands

Background: Efavirenz (EFV) treatment has been associated with an increased risk of neuropsychiatric adverse events.

Methods: In this double-blind placebo-controlled trial, 157 treatmentnaïve patients with HIV RNA $>5,000$ copies $/ \mathrm{mL}$, were randomised $1: 1$ to either etravirine (ETR) $400 \mathrm{mg}$ once daily $(\mathrm{n}=79)$, or EFV $600 \mathrm{mg}$ once daily $(\mathrm{n}=78)$, plus two NRTIs. At screening, baseline, Week 2, 6, and 12, subjects completed the HIV-Patient Symptoms Profile (HPSP) questionnaire on the impact of adverse events associated with their treatment. The domains were classified as CNS (attention/memory, dizziness, mood, pain, sleep, tiredness) or nonCNS (adherence, body shape, breathing, digestion, sexual health, skin, temperature, vision). For each domain, the mean of worst reported scores per patient, during visits at Week 2, 6 and 12, was compared between arms.

Results: Overall, the patients were $81 \%$ male, $85 \%$ Caucasian, with a median age 36 years. Baseline median CD4 Count was 302 cells $\mu \mathrm{L}$, median HIV RNA $4.8 \log _{10}$ copies/mL. In the primary analysis, $13 / 79$ patients $(16.5 \%)$ in the ETR arm, versus $36 / 78(46.2 \%)$ in the EFV arm, showed at least one Grade 1-4 treatment-emergent drug-related NPS AE $(p<0.001)$. In the analysis of the HPSP questionnaire, the mean score of the etravirine arm was significantly better than the efavirenz arm for the domains of dizziness, vision and sleep $(p<0.05$ for each comparison). For dizziness and sleep, the largest difference between arms was seen at Week 2, with smaller differences seen at Week 12. Differences in vision between the arms remained constant for Weeks 2-12. Patients with at least one neuropsychiatric adverse event had significantly worse scores in the CNS domains of the HPSP questionnaire $(\mathrm{p}<0.001)$. Patients with a medical history of neuropsychiatric adverse events had significantly worse scores in the CNS domain $(\mathrm{p}<0.001)$.

Conclusions: In the double-blinded SENSE trial, three patientreported adverse events with randomized treatment—dizziness, vision and sleep-were significantly worse in the efavirenz arm, compared to the etravirine arm.

\section{PO 29}

NEW SWITCH STRATEGY WITH RALTEGRAVIR ASSOCIATED TO BOOSTED PI IN HIV PATIENTS

S. Martini ${ }^{1}$, F. Di Martino ${ }^{1}$, A. Masiello ${ }^{2}$, A. Cascone ${ }^{1}$, A. Filippini ${ }^{1}$, P. Filippini ${ }^{l}$

${ }^{1}$ UOC Diagnosi e Terapia AIDS della AOU della Seconda Università degli Studi di Napoli, Naples, Italy; ${ }^{2}$ AORN G.Moscati, Avellino, Italy 
Introduction: The prognostic improvement in HIV patients, induced by HAART, has emphasized the problem of optimization of the therapy management. The advent of new drugs has allowed to prove new HAART regimens that may represent a valid option either in proactive switches than in viro-immunological failures.

AIM: Assessing if a HAART regimen NRTI-sparing, based on association of Raltegravir (RAL) and boosted PI (PI/r), may represent a good therapeutic option about efficacy, tolerability and adherence compared with Optimized Background Therapy (OBT) in switches for toxicity, simplification or failure.

Methods: We have enrolled 64 consecutive patients, treated for HIV infection, observed from January to December 2009. Among these patients, 13 have switched ( 7 for failure, 5 for intolerance, 1 for simplification) to a HAART regimen, based on association of $\mathrm{RAL}+\mathrm{PI} / \mathrm{r}$ (Cases); 51 were in OBT with association of $2 \mathrm{NRTI}+1$ $\mathrm{PI} / \mathrm{r}$ (Controls). HIV/HCV coinfection was in $46 \%$ of Cases and $35 \%$ of Controls. Laboratory tests have been performed (CD4, HIV-RNA, Triglycerides, Cholesterol, ALT, AST, Creatinine, Haemogram) to assess efficacy and tolerability. Adherence has been assessed by counseling and SERAD questionnaire (Self-Reported Adherence).

Results: Data of Cases have been compared to data pre-switch (PS) of the same group and to data of Controls. Mean CD4 count has been 649 in Cases, 542 in PS, 668 in Controls. About Cases, before switch, 10 patients had been treated with an association of $2 \mathrm{NRTI}+1 \mathrm{PI} / \mathrm{r}, 2$ patients had been treated with 2 NRTI +1 NNRTI, 1 patient was untreated. After switch, $31 \%$ of patients has suppressed its previous HIV viremia, $31 \%$ has reduced it, $23 \%$ has confirmed previous negativity, $15 \%$ has increased HIV-RNA. Hypertransaminasemia has been detected in $23 \%$ of Cases, $46 \%$ PS, $17 \%$ of Controls. High Triglycerides have been detected in $15 \%$ of Cases, $23 \%$ PS, $27 \%$ of Controls. High Cholesterol has been detected in 30\% of Cases, $46 \%$ PS, $45 \%$ of Controls. Our preliminary results show preserved efficacy of treatment with RAL and PI/r, compared to Controls, with increase of CD4 mean in Cases after switch. In Cases, lipid values have been resulted lower than Controls and reduced if compared to PS. In Cases, Hypertransaminasemia has appeared reduced, relative to PS, but it has showed higher incidence, compared to Controls. In this last group, however, there were less HIV/HCV coinfected patients (35 vs. $46 \%$ ). Conclusions: Our preliminary data suggest that a HAART regimen, based on association of RAL and PI/r, may represent a valid therapeutic option in patients who need a switch for failure, toxicity or poor adherence. This therapy would result effective, well tolerated, with low pill burden, allowing moreover to save either NRTI class than NNRTI one. In this way, it will be possible to preserve future therapeutic options, preventing cross resistance.

\section{PO 30}

SWITCHING TO A NRTI- AND RITONAVIR-SPARING THERAPY: A VALID OPTION IN HIV POSITIVE EXPERIENCED-PATIENTS

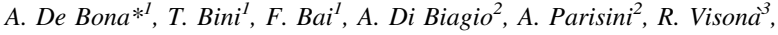

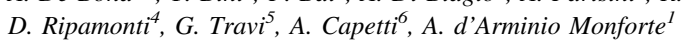

${ }^{I}$ Department of Medicine, Surgery and Dentistry, University of Milan, Clinic of Infectious Diseases, "San Paolo" Hospital, Milan, Italy; ${ }^{2}$ Infectious Diseases Clinic, University of Genoa, San Martino Hospital, Genoa, Italy; ${ }^{3}$ Department of Infectious Diseases, Busto Arsizio Hospital, Busto Arsizio, Italy; ${ }^{4}$ Division of Infectious Diseases, Ospedali Riuniti, Bergamo, Italy; ${ }^{5}$ Department of Infectious Diseases, Università Vita-Salute San Raffaele Scientific Institute, Milan, Italy; ${ }^{6}$ Department of Infectious Diseases of the Luigi Sacco Hospital, Milan, Italy

Background: HAART has reduced AIDS-related mortality and morbidity, but it may contribute to the increased incidence of metabolic disorders in HIV-infected patients (pts). Both NRTI class and boosted ritonavir are major responsibles of such toxicities. Thus, alternative therapeutic strategies are warranted. The aim of our study is to evaluate the immuno-virological and metabolic outcome of
HAART-experienced, HIV-infected pts switching to a NRTI and ritonavir-sparing regimen because of toxicity.

Methods: HIV pts, switching because of toxicity to ATV $200 \mathrm{mg}$ BID + RAL 200 mg BID, were enrolled in an observational, multicentre study. Inclusion criteria were: HIV-RNA $<50 \mathrm{cp} / \mathrm{ml}$, no history of primary PI mutations, RAL naïve and documented NRTI-or ritonavir-related toxicity. Pts with decompensated cirrhosis and/or on anti-acid therapy were excluded from the study. The primary endpoint was virological failure at T12 month (confirmed HIV-RNA $>50 \mathrm{cp} /$ $\mathrm{mL}$ ). As secondary endpoints immunological failure (defined as confirmed $20 \%$ decline in CD4+ T cell counts compared to baseline) and modification of metabolic parameters (triglycerides, total cholesterol, HDL, LDL, glycemia) were evaluated. Data were collected at the time of RAL/ATV switch (T0) and after 3 (T3), 6 (T6) and 12 (T12) months. Results were expressed as median and Interquartile Range (IQR). The comparison between study time points were performed by Wilcoxon test.

Results: 44 HIV pts were enrolled. The median age was 47 years (IQR 42-62), the majority of subjects were male $(26,59 \%)$, IVDU or MSM (17, 38.6\% each), with long history of HIV disease (median 198 months, IQR 134-269) and with long duration of HAART (median 148.5 months, IQR 167-190.5). Reasons for switch were: cardiovascular events $(25 \%)$, dyslipidemia or diabetes $(20 \%)$, renal or bone toxicity (20\%), other (35\%). In $25 \%$ of cases multiple toxicities were found. Before switching to ATV + RAL, 25 pts (57\%) were on boosted-PI, 10 (23\%) on unboosted ATV and 9 pts $(20 \%)$ on NNRTI based HAART. 25 pts (57\%) were on Tenofovir, 12 pts $(27 \%)$ on Abacavir, 4 pts (9\%) on AZT and 3 pts (7\%) on other back-bone. T3 was reached by 31 pts, T6 by 28 pts and T12 by 18 pts. No viroimmunological failures were observed during the study period. All the $18 \mathrm{pts}$ that reached the 12 month were still HIV-RNA $<50 \mathrm{cp} / \mathrm{ml}$. Median increase of CD4 count were 4.5 (IQR -60.5, 107) at T6 and 17 (IQR -65.5, 239.5) at T12. Overall we noted a significant reduction of triglycerides, albeit normal at baseline (T0: $156 \mathrm{mg} / \mathrm{dL}$, IQR 108-209; T3: 122 mg/dL, IQR 70-194, p = 0.002; T6: 111/mg/ $\mathrm{dL}$, IQR $81-175, \mathrm{p}=0.017$ ), and increase of HDL cholesterol at T3 (T0: $41 \mathrm{mg} / \mathrm{dL}$, IQR 26-50; T3: $46 \mathrm{mg} / \mathrm{dL}$, IQR 35-63; $\mathrm{p}=0.022$ ). Up to $39 \%$ (17/44) of pts showed hypertriglyceridemia at T0; in $65 \%$ (11/17) of pts triglyceride levels returned to normal range at T6.

Conclusions: Although preliminary, our data suggest that RAL/ATV therapy may represent a valid option in the management of HIVpositive pts with metabolic disorders or other toxicities.

\section{PO 31}

ANALYSIS OF EFFICACY AND TOLERABILITY OF RALTEGRAVIR-BASED REGIMENS BY BACKGROUND THERAPY IN SALVAGE: A MULTICENTRE ITALIAN EXPERIENCE

S. Landonio* ${ }^{\prime}$, A. Capetti ${ }^{1}$, P. Meraviglia ${ }^{2}$, A. Di Biagio ${ }^{3}$, S. Lo Caputo ${ }^{4}$, G. Sterrantino ${ }^{5}$, A. Ammassari ${ }^{6}$, B. Menzaghi ${ }^{7}$, M. Franzetti ${ }^{8}$, G. De Socio ${ }^{9}$, G. Pellicano ${ }^{10}$, E. Mazzotta ${ }^{11}$, S. Melzi ${ }^{l}$, P. Zucchi ${ }^{1}$, G. Rizzardini ${ }^{l}$

${ }^{1} 1$ st Division of Infectious Diseases, "Luigi Sacco" Hospital, Milan, Italy; ${ }^{2} 2$ nd Division of Infectious Diseases, "Luigi Sacco" Hospital, Milan, Italy;

${ }^{3}$ Infectious Diseases Clinic, "San Martino" Hospital, Genoa, Italy; ${ }^{4}$ Infectious Diseases Clinic, "Santa Maria Annunziata" Hospital, Florence, Italy; ${ }^{5}$ Division Infectious Disease, "Careggi" Hospital, Florence, Italy; ${ }^{6} 3 \mathrm{rd}$ Division of Infectious Disease, "Lazzaro Spallanzani" Hospital, Rome, Italy; ${ }^{7}$ Ospedale di Circolo, Division of Infectious Disease, "Ospedale di Circolo", Busto Arsizio, Italy; ${ }^{8}$ rd Division of Infectious Diseases, "Luigi Sacco" Hospital, Milan, Italy; ${ }^{9}$ Division of Infectious Diseases, "S Maria della Misericordia" Hospital, Perugia, Italy; ${ }^{10}$ Division of Infectious Disease, "Policlinico G Martino", Messina, Italy; ${ }^{11}$ Division of Infectious Disease, "Ospedale Civile Spirito Santo", Pescara, Italy

Background: Despite the official guidelines suggest to keep always NRTIs as a component of HAART, evolving concepts in salvage often move the clinicians to limit rescue regimens to active drugs as reported by genotypic tests. 
Objective: The aim of this analysis is to assess whether simpler rescue regimens are less effective on virus replication control and whether there are differences in toxicity using more or less complex associations.

Methods: Patients from the MK0518 EAP were followed-up prospectively since enrolment in the study. Clinical and laboratory data were collected every 2-4 months after commercialization. The cohort has been divided for the current analysis in three subgroups: dual regimens (A), triple/quadruple regimens (B) and megaHAART $(>4$ drugs, C). Statistical analysis has been performed using parametric and non-parametric tests.

Results: Most of the cohort patients were on triple/quadruple regimens $(186 / 253,74 \%)$. Group A $(\mathrm{n}=38), \mathrm{B}(\mathrm{n}=186)$ and C $(\mathrm{n}=29)$ were homogeneous by baseline HIV-RNA $(\mathrm{p}=0.29)$, and GSS ( $p=0.22$ ), while baseline CD4 levels showed a significant trend towards higher CD4 counts in simpler regimens (means: $\mathrm{A}=351$; $B=281$ and $C=164 / \mathrm{mmc}, p=0.0009$, Kruskall-Wallis test), reflecting a tendency in prescription. No statistically significant differences emerged in the rate of virologic failures or overall treatment discontinuations, and failure was not correlated with lower GSS scores, rather suggesting problems of adherence. Viral failure indeed was more frequent in more complex regimens $(\mathrm{A}=5 \%, \mathrm{~B}=11 \%$, and $\mathrm{C}=17 \%)$. All patients had impressive $\mathrm{CD} 4$ gains $(\mathrm{A}=+242$; $\mathrm{B}=+232 ; \mathrm{C}=+248, \mathrm{p}=0.52$, paired $t$ test). From a metabolic point of view, no difference was shown for ALT/AST levels, nor for triglycerides, while total cholesterol levels showed a trend to increase over time with more complex drug combinations. Patients on simpler regimens had significantly higher baseline cholesterol. Seven patients died in group B, four of non-Hodgkin's lymphoma (NHL), one of acute myocardial infarction, one of end stage liver disease and one post-transplantation for $\mathrm{HCV}$-related cirrhosis, one died in group $\mathrm{C}$ of hepatocellular carcinoma, and no one died in group A.

Table 1 Characteristics of the cohorts and immunological, virologic and metabolic evolution by W48 $(\mathrm{n}=153)$

\begin{tabular}{|c|c|c|c|c|}
\hline Parameters & $\begin{array}{l}\text { Baseline } \\
\text { values } \\
\text { (mean: A; } \\
\text { B; C) }\end{array}$ & $\mathrm{P}^{*}$ & $\begin{array}{l}\text { Delta values at } \\
\text { W48 (mean: A; } \\
\text { B; C) }\end{array}$ & $\mathrm{P}^{*}$ \\
\hline $\mathrm{CD} 4+\mathrm{T}($ cells $/ \mathrm{mmc})$ & $\begin{array}{l}351 ; 281 ; \\
164\end{array}$ & 0.0009 & $242 ; 232 ; 248$ & 0.52 \\
\hline $\begin{array}{l}\text { HIV RNA } \log _{10} \\
\text { (copies } / \mathrm{mL})\end{array}$ & $\begin{array}{l}3.67 ; 3.78 \\
\quad 4.16\end{array}$ & 0.29 & $\begin{array}{l}-1.89 ;-2.02 \\
\quad-1.96\end{array}$ & 0.69 \\
\hline ALT (IU/L) & $39 ; 43 ; 40$ & 0.36 & $+3 ; 0,-4$ & 0.72 \\
\hline AST (IU/L) & $37 ; 47 ; 48$ & 0.36 & $-7 ;-2 ;-1$ & 0.38 \\
\hline $\begin{array}{l}\text { Total cholesterol } \\
\quad(\mathrm{mg} / \mathrm{dL})\end{array}$ & $\begin{array}{l}200 ; 183 ; \\
161\end{array}$ & 0.01 & $+10 ;+18 ;+37$ & 0.000 \\
\hline $\begin{array}{l}\text { Triglycerides } \\
(\mathrm{mg} / \mathrm{dL})\end{array}$ & $\begin{array}{l}226 ; 237 ; \\
\quad 213\end{array}$ & 0.53 & $-25 ;-17 ;-31$ & 0.56 \\
\hline
\end{tabular}

Conclusions: Simpler regimens were neither associated in our analysis with a higher risk of viral failure nor to an impaired CD4+ T cell increase. The metabolic impact of simpler regimens was not related to an important gain in the toxicity profile, except for a slightly reduced increase in cholesterol levels. This analysis will be repeated at 96 weeks to assess long-term tolerability.
PO 32

IN VITRO CELL TOXICITY AND MATRIX METALLOPROTEINASE9 INHIBITION BY ANTIRETROVIRAL DRUGS IN CULTURED PRIMARY ASTROCYTES

G.M. Liuzzi ${ }^{*}{ }^{1}$, T. Latronico ${ }^{1}$, E. Raimondo ${ }^{1}$, G. Di Bari ${ }^{1}$, F. Mengoni ${ }^{2}$, M. Lichtner ${ }^{4}$, P. Riccio ${ }^{2}$, V. Vullo ${ }^{3}$, C.M. Mastroianni ${ }^{4}$

${ }^{I}$ Department of Biochemistry and Molecular Biology, University of Bari, Bari, Italy; ${ }^{2}$ Department of Biology D.B.A.F., University of Basilicata, Potenza, Italy; " "La Sapienza" University, Rome, Italy; "La Sapienza" University, Polo Pontino, Latina, Italy

Background: The toxic effects of new antiretroviral drugs on the central nervous system (CNS) are unclear. Because these drugs penetrate the brain even at low concentrations, it becomes crucial to determine the doses which can be toxic for the CNS resident cells. Moreover, after the recent introduction into clinical practice, it is unclear whether the efficacy of the antiretroviral drugs of new generation may also derive from their ability to exert extravirological effects on factors responsible for the development of HIV brain injury, e.g. matrix metalloproteinases (MMPs).

Objective: To investigate on the toxicity of four different antiretroviral drugs and their ability to modulate the activity of MMPs in astrocyte cultures.

Methods: Primary cultures of rat astrocytes were activated by exposure to 10 ?g/ml lipopolysaccaride (LPS) (positive control) and simultaneously treated for $20 \mathrm{~h}$ with increasing doses (1-5-10-25. 50 ?M) of: Efavirenz (EFV); Darunavir (DRV); Maraviroc (MVC) or Raltegravir (RAL). Culture supernatants were subjected to gelatin zymography for the assessment of MMP-2 and MMP-9 protein levels. Quantitative determination of MMP-9 and MMP-2 was done by computerized scanning densitometry. Single drug toxicity was assessed by the MTT test. Each drug was considered toxic at the concentration able to induce a percentage of cell survival above $60 \%$. Results: The treatment with antiretroviral drugs inhibited MMP-9 levels in LPS-activated astrocytes in a dose-dependent manner, while no statistically significant changes of MMP-2 levels were observed. In particular, a statistically significant inhibition of MMP-9 was observed when astrocytes were treated with 25 ?M EFV $(60 \%$ of inhibition) or with 50 ?M RAL (40\% of inhibition). As assessed by the MTT test, the toxicity of the antiretrovirals ranges from 10 and 50 ?M. In particular, EFV was toxic for astrocytes at the concentration of 25 ?M, MVC at 10 ?M, while DRV and RAL were toxic at the concentration of 50 ?M.

Conclusions: The present results indicate that EFV and RAL directly inhibit MMP-9 levels in LPS-activated astrocytes with mechanisms that are independent from their antiviral activity. The toxic doses of antiretrovirals are much higher than those found in the CSF of HIVpositive patients. Our results highlight some beneficial/deleterious extra-viral effects of the antiretroviral drugs that may be useful to improve the development of new therapeutic strategies for the management of HIV infection.

\section{PO 33}

RENAL TOXICITY FOR SWITCHING FROM TENOFOVIR/ EMTRICITABINE (TDF/FTC) TO ABACAVIR/LAMIVUDINE (ABC/3TC): AN ASSOCIATION TO BE CLARIFIED

L. Manzini ${ }^{*}$, V. Borghi ${ }^{1}$, S. Rusconi ${ }^{2}$, B. Vergani ${ }^{2}$, P. Bonfanti ${ }^{3}$, L. Calza ${ }^{4}$,

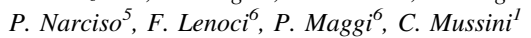

${ }^{I}$ Clinica di Malattie Infettive Policlinico di Modena, Modena, Italy; ${ }^{2}$ Istituto di Malattie Infettive Università di Milano, Ospedale L. Sacco, Milan, Italy; ${ }^{3}$ Dipartimento di Malattie Infettive Ospedale L. Sacco, Milan, Italy; ${ }^{4}$ Dipartimento di Malattie Infettive Università di Bologna, Bologna, Italy; ${ }^{5}$ I.N.M.I. "L. Spallanzani", Roma; ${ }^{6}$ Dipartimento di Malattie Infettive Università di Bari, Bari, Italy 
Background: The impact on renal toxicity of switching from tenofovir/emtricitabine (TDF/FTC) to abacavir/lamivudine (ABC/3TC) is still unclear.

Methods: A multicenter retrospective cohort study was conducted on HIV+ patients (pts) who stopped TDF and started ABC for renal adverse events. The switch could have been based on an increase in creatinine and/or serum creatinine ( $\mathrm{SCr}$ ) clearance, proteinuria, hypophosphoremia. Modifications of kidney function were recorded 1-36 months after switch.

Results: 83 pts switched to ABC for TDF-associated renal toxicity. Median age was 41 years (IQR 38-53.5), 65 (75.6\%) pts were males, $90.7 \%$ was Caucasian, $62(72.1 \%)$ had a sexual HIV transmission. Among co-pathologies 19 pts (22.1\%) had hypertension, $43 \%$ were smokers, 21 had HCV co-infection (24.4\%), 6 HBV co-infection (7\%), and 27 had a previous AIDS diagnosis (31.4\%). CD4 nadir was 202 cells/ $\mu \mathrm{L}$ (IQR 70-288) and HIVRNA was $5.07 \mathrm{log}$ copies $/ \mathrm{ml}$ (IQR 4.81-5.45). TDF was co-administrated with 3TC in $38.4 \%$ of the cases and with FTC in $51.2 \%$. $71 \%$ was on a protease inhibitorbased regimen and $29 \%$ on a non-nucleoside.

Reasons for switching were: proteinuria in 42 pts (48.8\%), and increased $\mathrm{SCr}$ or hypophosphoremia in $41 \mathrm{pts}(47.7 \%)$. Baseline $\mathrm{SCr}$ was 0.91 (IQR 0.8-1.17), GFR was 84.62 (62.3-107.9) and phosphoremia (available in only 37pts) was $3.4 \mathrm{mg} / \mathrm{dL}$ (IQR $2.6-3.7$ ). In 42 pts with proteinuria, spot values were $40 \mathrm{mg} / \mathrm{dL}$ (IQR 25-100) but $24 \mathrm{~h}$-proteinuria (available in $18 \mathrm{pts}$ ) was $885 \mathrm{mg} / \mathrm{die}$ (IQR 443-1,737). The renal damage was observed after 14 months (IQR 1-25) of TDF treatment. One, 3 and 6 months after the switch, $\mathrm{SCr}$ and GFR had no significant changes, while proteinuria improved significantly ( $\mathrm{p}=0.001$ Wilcoxon test).

Conclusions: The present study shows that clinicians are worried about TDF renal toxicity and they switched the drug early even in presence of a $\mathrm{SCr}$ value slightly increasing but still in the normal range. Proteinuria improved significantly even in this short time of observation and among these pts not very compromised

\section{PO 34}

SMOKING AS A RISK FACTOR FOR DETERIORATION OF RENAL FUNCTION IN A COHORT OF HIV-NAÏVE PATIENTS TREATED WITH TENOFOVIR

A. Uglietti ${ }^{* 1}$, C. Gervasoni ${ }^{2}$, S. Di Giambenedetto ${ }^{3}$, E. Gabrielli $^{2}$, R. Cauda ${ }^{3}$, R. Esposito ${ }^{4}$, P. Grima ${ }^{5}$, G. Di Perri ${ }^{6}$, R. Maserati ${ }^{1}$, M. Galli $^{2}$

${ }^{I}$ Clinica di Malattie infettive, Fondazione IRCCS Policlinico San Matteo,

Pavia, Italy; ${ }^{2}$ Clinica di Mlattie Infettive, Ospedale L. Sacco, Milan, Italy; ${ }^{3}$ Clinica di Malattie Infettive, Università Cattolica del Sacro Cuore, Rome, Italy; ${ }^{4}$ Clinica di Malattie Infettive, Policlinico Universitario, Modena, Italy; ${ }^{5}$ Malattie Infettive, Ospedale S. Caterina Novella, Galatina, Italy; ${ }^{6}$ Clinica Malattie Infettive, Ospedale Amedeo di Savoia, Turin, Italy

Background: Cigarette smoke is the most preventable cause of death and chronic disease (atherosclerosis and cancer) in most countries and recent epidemiologic studies suggest that cigarette smoke promotes the progression of kidney disease. Smoking may cause renal injury through several hemodynamic actions and also non-hemodynamic actions of nicotine, such as damage to endothelial cells, interference with the coagulation/fibrinolysis systems, generation of oxygen radicals. Aim of this study was to evaluate Glomerular Filtration Rate (GFR) changes in relationship with smoking in an Italian cohort receiving a tenofovir (TDF)-containing HAART.

Methods: OSMA-1 (Observational Study on Metabolic Abnormalities), a multicenter Italian study, was designed to evaluate since February 2008 the efficacy and the safety of TDF-based regimen in a real-life clinical setting. HIV infected, therapy naïve subjects were enrolled. GFR was estimated using Cockcroft-Gault (CG), Modification of Diet in Renal Disease (MDRD) and Chronic Kidney Disease Epidemiology Collaboration (CKD-EPI) equations. Statistical analyses used a parametric test and a mixed model was used to analysis changes from baseline to 6,12 , and 24 months, with the different variables as fixed effect plus visit (categorical) and baseline value (continuous as covariate).

Results: We consecutively enrolled 172 patients (91.3\% Caucasian; $72.2 \%$ males; mean age 39.3 years; $48.8 \%$ heterosexual; $36.6 \%$ smokers). At baseline median CD4+ cell count was 225 cells $/ \mu \mathrm{L}$ (range 2-701), HIVRNA $>100,000$ copies $/ \mathrm{mL}$ in $43.6 \%$. Median body mass index (BMI) was 22.6 for males and 21.9 for females. A boosted PI was given in $60.5 \%$ of cases. At 6-months evaluation, women (W) had greater declines in GFR versus men (M), independently from the GFR equation used (means in $\mathrm{W}$ vs. $\mathrm{M}$ for $\mathrm{CG}$, MDRD and CK-EPI, respectively: $-7.2 ;-12.1 ;-10$ vs. $0.5 ;-1.3$; $0.3 \mathrm{~mL} / \mathrm{min} / 1.73 \mathrm{~m}^{2}, \mathrm{p}=0.0001-0.0026$ ). No further decline was documented after 6 months. However, in multivariate analyses smoking is associated with a renal function worsening (CG, MDRD and CK-EPI: $p=0.073, p=0.012$ and $p=0.05$, respectively).

Conclusion: Smoking, beyond female gender, is an important renal risk factor in patients treated with a combination therapy containing TDF. Clinicians should prioritize any efforts to encourage their patients quit smoking as the most cost-effective and beneficial strategy to spare also a possible renal toxicity associated with TDF.

\section{PO 35}

SAFETY OF FOS-AMPRENAVIR IN HIV INFECTED PATIENTS WITH CO-MORBIDITIES: A RETROSPECTIVE COHORT ANALYSIS

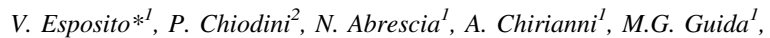
G. Parrella ${ }^{l}$

${ }^{I}$ AO Monaldi-Cotugno-CTO, Naples, Italy; ${ }^{2}$ Department of Medicine and Public Health, Second University of Naples, Naples, Italy

Background: As the HIV-population grows older, infectious disease specialists have to consider unfamiliar areas of internal medicine such as lipid-lowering therapy and smoking cessation. Moreover, the ART regimen itself may be a modifiable risk factor because of concern about potential increases among HIV-infected patients in liver toxicity and vascular risk attributable to dyslipidemia, insulin resistance, fat redistribution, and hypertension, we hypothesized that fos-amprenavir would be associated with few metabolic and hepatic side effects.

Methods: We set up an observational single arm retrospective study on a cohort of 137 HIV infected patients, followed up at A.O.R.N. Cotugno Hospital, Naples, Italy, attending the third and the fourth division of infectious diseases, in treatment with an antiretroviral regimen including Fosamprenavir. Primary endpoint: Proportion of subjects experiencing at least one of the following condition during the follow-up: grade $=3$ in ALT, grade $=3$ in total cholesterol, grade $=3$ in triglycerides, grade $=3$ in serum glucose level, treatment discontinued for AEs. Secondary endpoints: ? Proportion of subjects experiencing at least one of the above mentioned conditions at the end of the follow up. ? proportion of subjects requiring cotherapy discontinuation. ? proportion of subjects with $\mathrm{VL}<50 \mathrm{cp} / \mathrm{ml}$ after 24 and 48 weeks of treatment

Results: The follow up period was up to 48 weeks in most patients (114 patients; $83.21 \%$ ) with a minimum of 24 weeks in the remaining cases. Only seven patients met the criteria to reach the primary endpoint and no one of them discontinued HAART therapy during the follow up period, without any statistically significant differences between naïve and experienced and according to age ( $>50$ years old) and backbone. Seventy-six percent of patients presented HIV RNA $<50 \mathrm{cp} / \mu \mathrm{L}$ after 24 weeks of therapy and $80 \%$ reached viral load $<50 \mathrm{cp} / \mu \mathrm{L}$ at 48 weeks of observation. The mean increase of CD4+ cell was about 200 cells $/ \mu \mathrm{L}$. Looking at secondary endpoints at the end of follow up, we failed to find any patients with fasting cholesterolemia above grade 3 , while 2 out of $114(1.75 \%)$ cases presented AST and or ALT $>$ grade 3, one patient out of $112(0.89 \%)$ had fasting triglyceridemia $>$ grade 3 and 1 out of 114 cases had fasting 
glycaemia $>$ grade 3 . One patient developed a malignant neoplasm $(0.7 \%)$ and $4(3 \%)$ displayed a newly diagnosed hypertension. All therapies administrated for this last comorbidities were well tolerated and did not need any changes or discontinuation related to HAART therapy.

Conclusions: Our retrospective study showed that fosamprenavir based regimens had a reduced number of serious metabolic adverse events during a 1 year follow up period, with a low incidence of comorbidities and satisfying results in term of viro-immuological response also in patients with already existing co-morbidities requiring other therapies.

\section{PO 36}

EFFICACY AND SAFETY OF ATAZANAVIR/RITONAVIR THERAPY IN A COHORT OF TREATMENT-EXPERIENCED PATIENTS

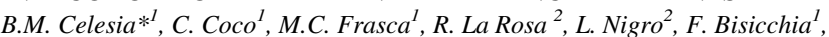
F. Palermo ${ }^{I}$, G. Nunnari ${ }^{I}$, M.T. Mughini ${ }^{I}$

${ }^{I}$ U.O. Malattie Infettive Università di Catania ARNAS Garibaldi Catania, Catania, Italy; ${ }^{2}$ U.O. Malattie Infettive P.O. Ferrarotto Azienda Policlinico OVE, Catania, Italy

Background: Atazanavir (ATV) based regimens are generally well tolerated and seem to have a better metabolic profile than other PIs and NNRTIs based treatment. Few study assessed safety and effectiveness of ATV/r regimens for more than 2 years. Aim of this study is to describe efficacy and safety of ATV/r based regimens in a cohort of experienced patients treated for a long period of time.

Methods: Patients switched to ATV300/r based regimen after at least 6 months of any other HAART therapy were enrolled in a retrospective study. We evaluated the proportion of patients maintaining HIV RNA $<50$ copies/ml, median increase of CD4 cell count, total cholesterol and triglycerides, liver transaminases, total bilirubin at the time of starting the ATV/r therapeutic regimen and after 1-4-8-12-24-36-48-60 months respectively. More frequent side effects and causes for discontinuation were also described.

Results: A total of 107 patients were evaluated; 62 (57.9\%) were male, median age 45 (IQR 40-54) years, 55\% heterosexuals, 24\% MSMs, $21 \%$ IVDUs. $32 \%$ were HBV and/or HCV coinfected. Median time from HIV diagnosis was 11 years (IQR 5-15). Median length of HAART was 8 (IQR 4-11) years; median number of line of treatment was 4 (IQR 2-5). Before switching to ATV/r, $46 \%$ were on PI/r based treatment, $30 \%$ on NNRTI, $15 \%$ on PI unboosted and $9 \%$ on triple NRTI. At baseline median CD4 cell count was 341 (IQR 237-537) cells $/ \mu 1$, median HIV RNA viremia $\log 2.45 \pm 1.47$ copies $/ \mathrm{ml} ; 58 \%$ had HIV RNA $<50$ copies/ml. Median total cholesterol (TC) was 205 (IQR 171-269) $\mathrm{mg} / \mathrm{dl}, 55 \%$ with more than normal value, median triglycerides 174 (IQR $116-315) \mathrm{mg} / \mathrm{dl}, 59 \%$ with more than normal value. After 12, 24, 36 months of treatment median CD4 cell count was 449 (IQR 300-615), 482 (IQR 354-700), 493 (IQR 368-773) cells/ $\mu$ respectively. At the same times $83,86,79 \%$ of patients on treatment had HIV RNA $<50$ copies/ml. After 12 months $57 \%$ had altered TC value, $51 \%$ altered triglycerides. Patients with elevated bilirubinemia ( $>1.5 \mathrm{mg} / \mathrm{dl}$ ) were $83,84,73 \%$ after 4,8 and 12 months of treatment. After a median time of follow up of 40.6 months (IQR 24.3-59.8) 17\% stopped the treatment. The most frequent causes for discontinuation were virological failure $(8.5 \%)$ patient's choice $(5.7 \%)$ toxicity $(2.8 \%)$.

Conclusions: In a clinical real life setting ARV regimens based on ATV/r showed sustained virological response also after 36 months of treatment in an high proportion of patients. Treatment was generally well tolerated and safe although just few patients achieved a significant reduction of lipids levels. Hyper-bilirubinemia was frequent but never caused discontinuation.
PO 37

HAART MODIFICATION IN VIROLOGICALLY SUPPRESSED PATIENTS

F. Castelnuovo ${ }^{1}$, A. Bergamasco ${ }^{1}$, R. Allegri ${ }^{l}$, I. El Hamad ${ }^{1}$, A. Scalzini ${ }^{l}$

${ }^{I}$ Department of Infectious Diseases, 1 Division, Spedali Civili, Brescia, Italy

Objectives: The aim of the study was to determine in an Italian cohort the incidence of treatment switches and their causes to modify the highly active antiretroviral therapy (HAART). Treatment modification can be either proactive (prevention) when the goal is to avoid an adverse event or drug interaction or to maximize the potential for optimal adherence and reactive (complication) when the modification is made after the occurrence of an adverse event or drug interaction. Methods: We included in the study the outpatient population on antiretroviral therapy of the 1 Division of Infectious Diseases in a 3 years (2008-2010) follow up period. The endpoints were substitution or discontinuation of at least one HAART component of the regimen for any reason (poor adherence, simplification and intolerance/toxicity). Switches were done with a new drug only in patients without resistant virus. When a patient discontinued a drug in the antiretroviral regimen, regardless of whether or not he switched to another regimen, clinicians were asked to report the reason for interruption (only one reason for each stopped drug). Descriptive analysis was performed.

Results: A total of 897 patients were included in the study: $28.5 \%$ were female, $44 \%$ were $\mathrm{HCV}$ coinfected; their median age was 45 years. The proportion of patients achieving undetectable HIV-1 RNA was near $90 \%$. The main causes of switching (see Table) were the simplification/adherence (181) and metabolic complications (48) in a 3 years follow up period. In 257 patients (70\%) the NRTIbackbone was changed, in general, within-class substitutions using a newer agent and coformulated drugs or to prevent/interrupt toxicity. Switches with PIs or NNRTI $(110 ; 30 \%)$ were done to reduce dosing frequency, pill count, drug-drug interactions, or for metabolic and gastrointestinal/hepatic complications.

\begin{tabular}{lllll}
\hline Variables and year & 2008 & 2009 & 2010 & Total \\
\hline Prevention no (\%) & 60 & 119 & 45 & 224 \\
Simplification/adherence & 52 & 88 & 41 & $181(80 \%)$ \\
Risk for cardiovascular disease & 4 & 18 & 2 & $24(11 \%)$ \\
Risk for toxicity & 3 & 11 & 1 & $15(7 \%)$ \\
Pregnancy & 1 & 2 & 1 & $4(2 \%)$ \\
Complication no (\%) & 48 & 54 & 41 & 143 \\
Metabolic & 15 & 20 & 13 & $48(33 \%)$ \\
Haematologic & 6 & 14 & 2 & $22(16 \%)$ \\
Renal & 6 & 12 & 4 & $22(16 \%)$ \\
Gastrointestinal & 12 & 2 & 6 & $20(14 \%)$ \\
Hepatic & 1 & 6 & 6 & $13(9 \%)$ \\
Hypersensitivity & 7 & - & 3 & $10(7 \%)$ \\
Neurological & - & - & 5 & $5(3 \%)$ \\
Others & 1 & - & 2 & $3(2 \%)$ \\
\hline
\end{tabular}

Conclusions: It seems important to evaluate reason-specific trends in the incidence of discontinuation in order to better understand the determinants of changes over time. The incidence of discontinuation for intolerance/toxicity is not declined over time while simplification strategies have become more frequent in recent years. 


\section{PO 39}

METABOLIC PROFILE IN A COHORT OF HIV-1 POSITIVE PATIENTS TREATED WITH RAL-BASED SCHEME: RESULTS AFTER 48 WEEKS

P. Corsi ${ }^{*}$, C. Martinelli ${ }^{I}$, A. Carocci ${ }^{1}$, F. Leoncini ${ }^{l}$

Infectious Diseases Unit AOU Careggi, Florence, Italy

Background: In patients with HIV infection, both naïve and experienced, Raltegravir (RAL) was an effective drug and generally well tolerated in antiretroviral combination therapy. This drug is certainly interesting to be taken into account in patients requiring cholesterollowering therapy or shift in those who are familiar or genetic predisposition to dyslipidemia. We have considered HIV seropositive patients receiving raltegravir as part of a combination regimen of second line or after multifailure, in particular we have evaluated the metabolic abnormalities.

Materials and methods: As a simplified metabolic profile were considered blood glucose, total cholesterol and fractions, triglycerides in 44 patients referred to our center who started combination therapy with raltegravir. The determination was evaluated at 48 weeks of RAL therapy. Of these patients 11 were females and 33 males. The mean age of patients was equal to 51 years (range 25-69 years) with as many as 37 patients ( $84 \%$ ) aged $>45$ years. The quantitative viral load was $<15$ copies/mL in 32 patients $(73 \%)$, in 9 (21\%) was $<50$ copies $/ \mathrm{mL}$ while in only 3 patients the viral load was not completely suppressed. The immunological balance of these patients was on average equal to $509 \mathrm{CD} 4 / \mathrm{mmc}$ (range 125-1,396).

Results: The metabolic profile of patients are summarized below.

Table 1 The metabolic profile in 44 patients-RAL

\begin{tabular}{lll}
\hline & Mean values & $\begin{array}{l}\text { Range values of } \\
\text { standard reference }\end{array}$ \\
\hline Blood glucose & $0.97(0.71-1.63)$ & $0.65-1.1 \mathrm{~g} / \mathrm{L}$ \\
Total cholesterol & $215(57-287)$ & $160-220 \mathrm{mg} / \mathrm{dL}$ \\
LDL cholesterol & $131(8-196)$ & $60-190 \mathrm{mg} / \mathrm{dL}$ \\
HDL cholesterol & $41(9-73)$ & $>45 \mathrm{mg} / \mathrm{dL}$ \\
Triglycerides & $226(49-208)$ & $50-170 \mathrm{mg} / \mathrm{dL}$ \\
\hline
\end{tabular}

Table 2 Number of RAL patients with altered values

\begin{tabular}{lll}
\hline Blood glucose & $>1.1 \mathrm{~g} / \mathrm{L}$ & $6 / 44$ \\
Total Cholesterol & $>220 \mathrm{mg} / \mathrm{dL}$ & $15 / 44$ \\
HDL cholesterol & $<45 \mathrm{mg} / \mathrm{dL}$ & $20 / 44$ \\
LDL cholesterol & $>190 \mathrm{mg} / \mathrm{dL}$ & $4 / 44$ \\
Triglycerides & $>170 \mathrm{mg} / \mathrm{dL}$ & $18 / 44$ \\
\hline
\end{tabular}

Conclusions: At 48 weeks, in patients previously experienced, the raltegravir based therapy has shown excellent results in our patients with regard to LDL cholesterol and blood glucose. The drug was well tolerated and also the control of viral suppression is confirmed.

\section{PO 40}

ONE-PILL ONCE A DAY HAART: SIMPLIFICATION STRATEGY MAINTAINING ADHERENCE, EFFICACY AND IMPROVING PHYSICAL AND PSYCHOLOGICAL OUTCOMES IN A COHORT OF HIV-INFECTED ADOLESCENTS AND YOUTHS

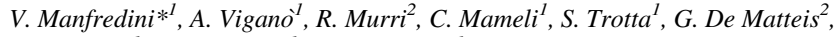
F. Di Nello ${ }^{l}$, V. Giacomet ${ }^{l}$, G.V. Zuccotti ${ }^{l}$

${ }^{1}$ Pediatric Clinic, L. Sacco Hospital, University of Milan, Milan, Italy;

${ }^{2}$ Department of Infectious Diseases, Catholic University of Rome, Rome, Italy
Background: Data on the effect of a reduced number of pills on efficacy and quality of life (QoL) in HIV-infected adolescents and youths are lacking.

Methods: Observational, prospective, cohort study including $30 \mathrm{HIV}$ vertically-infected adolescents and youths aging 14-25 years. Patients chronically treated (mean duration: 64 months) with lamivudine, tenofovir (TDF) and efavirenz (EFV) and with stable HIVRNA $<50 \mathrm{cp} / \mathrm{ml}$ were switched to a single-tablet regimen (STR) of emtricitabine + TDF + EFV. Data were collected with an anonymous 36-items questionnaire on self-reported adherence, quality of life and symptoms and with the Center for Epidemiologic Studies Short Depression Scale (CES-d 10). CD4 T-cell count, HIV-RNA and clinical parameters were assessed along with the questionnaire. Analysis of collected data at the switch $(\mathrm{t} 0)$, one $(\mathrm{t}+1)$ and 12 $(t+12)$ months after are reported.

Results: Thirty HIV-infected patients were enrolled; three were lost between $\mathrm{t} 1$ and $\mathrm{t} 12$, one because of adverse events and two because of reluctance to fill out the questionnaires. At baseline, the majority (86.7\%) of patients was asymptomatic; CDC classifications were $\mathrm{N}+\mathrm{A}$ in $43.3 \%$ and $\mathrm{B}+\mathrm{C}$ in $56.7 \%$ of cases. During the entire study period, all cases maintained HIV-RNA $<50$ copies $/ \mathrm{ml}$ and low mean rate of symptoms score (range $0-100$ ) between 9.8 and 6.8. CD4 T-cell \% showed a significant $(\mathrm{p}<0,05)$ increase from $35.2(\mathrm{SD} 8.9)$ to 39.4 (SD 14.1) between $\mathrm{t} 0$ and $\mathrm{t} 1$ and this improvement persisted at t12 (41.1, SD 14.3). Adherence, high above $80 \%$ at t0, remained stably optimal along the entire study period. Satisfaction with therapy score (range $0-10)$, high at baseline (8.2, SD 1.9), showed a persistent increase reaching significance $(\mathrm{p}=0.02)$ at $\mathrm{t} 12(9.0, \mathrm{SD} 1.4)$. Selfperceived physical fatigue declined from 3.6 (3.1) to 3.2 (2.9) at $\mathrm{t} 1$; a further slight decrease to 2.1 (2.2) was observed at t12. Self-perceived psychological fatigue also significantly $(\mathrm{p}=0.03)$ decreased between t0 (4.3, SD 3.1) and t1 (3.1, SD 2.2); this improvement persisted at t12. CES-d score (range 0-30) significantly $(p=0.04)$ decreased between t0 and t1 from 11.9 (3.3) to 10.7 (2.6) and the improvement was conserved at $\mathrm{t} 12$.

Conclusions: Simplification of a cart regimen to an STR consisting of efavirenz, emtricitabine and tenofovir maintains virological suppression, slightly improves immunological response, self-perceived physical and psychological fatigues, reduces depression and increases satisfaction with therapy.

\section{PO 41}

RESPONSES TO COMMON VACCINES IN ADOLESCENTS WITH HIV ACQUIRED PERINATALLY

R. Rosso ${ }^{I}$, L. De Hoffer ${ }^{I}$, A. Parisini ${ }^{1}$, L.A. Nicolini ${ }^{I}$, G. Secondo ${ }^{I}$, L. Taramasso ${ }^{1}$, E. Nicco ${ }^{1}$, A. Di Biagio ${ }^{1}$, C. Viscoli $^{1}$

${ }^{I}$ Department of Infectious Diseases, University of Genoa at San Martino Hospital, Genoa, Italy

Background: We investigated the coverage of hepatitis B virus (HBV) and measles, mumps, rubella with the addition of chickenpox (MMR-V) vaccination by measuring antibody titers, in adolescents with HIV acquired perinatally.

Methods: We retrospectively identified 39 adolescents who had undergone the mandatory vaccination for HBV. We assessed the age and the doses at which the vaccination was given and the levels of antibodies against HBs Ag (Monolisa HBs Ag Ultre Assay-Bio.Rad laboratories). The same data were analyzed with respect to MMR-V immunization.

Results: Thirty-nine patients (91\%) of the 43 followed by our center with a mean age of $17.53 \pm 0.7$ years. Thirty-five patients $(89 \%)$ were suppressed (HIV-RNA below 50 copies/mL), thirty (76\%) had a count of CD4+ over 501 cells $/ \mathrm{mmc}$. Patients had received an average of 3.80 doses of HBV vaccine (range 1-8). With regard to the antibody titre against HBV (HBsAb), 27 patients $(69 \%)$ had a titre $<10 \mathrm{mUI} / \mathrm{ml}$, and only $12(31 \%)$ had titre $>10 \mathrm{mUI} / \mathrm{ml}$. About 
MMR-V vaccine: 14 patients $(36 \%)$ have not performed any vaccination; 3 (8\%) performed the single measles vaccine; $22(56 \%)$ have played the full MMR-V vaccination. In this latter group, only nine (40\%) performed the two recommended boosters, but the age at which these subjects have been vaccinated does not reflect in any way the vaccination schedule. Regarding to antibody titres we have evaluated for both IgG and IgM MMR-V: IgM were negative in all patients. Only ten patients $(26 \%)$ had measles IgG positive; eight of them performed the MMR-V vaccine, but only three of these eight (37\%) had carried out the two recommended doses. Mumps IgG were found in one patient $(2 \%)$ who had also performed the double dose of MMR-V. Thirteen patients (33\%) had positive IgG for rubella, nine of which had made the vaccination [only $3(33 \%)$ with two boosters]. Finally, only eight patients $(20 \%)$ had an antibody response against chickenpox, but even among these, only four (50\%) had carried out the vaccination with two doses.

Conclusions: Despite the low number of patients, was interesting observe that age at which these subjects have been vaccinated and booster doses are the most varied and may not reflect in any way the Italian vaccination schedule. On the basis of these findings, may be useful to monitor that: the vaccination schedule was successfully completed, the antibody titres are protective against infectious diseases with highest impact now that the immunovirological answer through cART is optimal in most cases and allows us to maintain protective antibody titers.

\section{PO 42}

SUCCESSFUL USE OF A DARUNAVIR/RITONAVIR IN FIVE PREGNANT WOMEN: PHARMACOKINETICS, EFFICACY AND SAFETY

J. Ivanovic ${ }^{* 1}, R$. Bellagamba ${ }^{1}, M$. Tempestilli ${ }^{1}, F$. Signore ${ }^{2}$, C. Vallone $^{2}$, G. Pisani ${ }^{2}$, E. Nicastri ${ }^{1}$, C. Tommasi ${ }^{1}$, A.L. Gallo ${ }^{1}$, R. Fezza ${ }^{l}$, L.P. Pucillo ${ }^{1}$, P. Narciso $^{l}$

${ }^{I}$ National Institute for Infectious Diseases 'Lazzaro Spallanzani', Rome, Italy; ${ }^{2}$ Department of Obstetrics and Gynaecology, 'San Camillo-Forlanini'

Hospital, Rome, Italy

Background: Pregnancy could cause a significant reduction of plasmatic drug concentration of some protease inhibitors (PI). Furthermore, the protease inhibitors transplacental passage and compartmental diffusion are generally very low. Although, there is positive experience of darunavir (DRV) prescription during pregnancy, data concerning DRV pharmacokinetics and placental transfer are scant and secondary compartments concentrations are unknown. We report pharmacokinetics and compartmental distribution of once daily administrated DRV boosted with low-dose ritonavir (DRV/r), in combination with emtricitabine/tenofovir (FTC/TDF). In one experienced patient only, DRV was administrated twice daily and was associated to FTC/TDF and raltegravir (RAL).

Methods: HIV positive pregnant women on DRV/r as part of their routine maternity care were enrolled for the study. DRV plasma trough concentrations (Ctrough) were determined in the first (T1) and/or second (T2) and/or third trimester (T3) and at delivery (D) using a validated HPLC-UV methodology with a limit quantification of $78 \mathrm{ng} / \mathrm{ml}$. Cord blood and amniotic fluid concentrations were also measured at delivery. $12 \mathrm{~h}$ pharmacokinetic (PK) curves were performed between 33 and 37 weeks of gestation and in postpartum (PP) in one woman with twice daily administration. Newborn data including gestational age, weight, Apgar score, HIV infection status and any kind of maternal and/or newborn complication were collected at birth.

Results: Five Caucasian women with mean age of $34 \pm 2$ years and mean BMI of $31 \pm 10 \mathrm{~kg} / \mathrm{m}^{2}$, were recruited. All woman were virally suppressed (HIV RNA $<40 \mathrm{cpm}$ ) throughout pregnancy and had CD4 cell counts $>300$ cells $/ \mathrm{mm}^{3}$ (mean $674 \pm 268$ cells $/ \mathrm{mm}^{3}$ ). Mean DRV concentrations in T1 was $2,800 \mathrm{ng} / \mathrm{ml}$, in T2 (mean time after dosing $11 \pm 0.8 \mathrm{~h}$ ) was $1,769 \pm 153 \mathrm{ng} / \mathrm{ml}$, in $\mathrm{T} 3$ (mean time after dosing $11 \pm 0.4 \mathrm{~h}$ ) was $1,764 \pm 641 \mathrm{ng} / \mathrm{ml}$ and at delivery (mean time after dosing $7.25 \pm 3.5 \mathrm{~h}$ ) was $2,014 \pm 1,549 \mathrm{ng} / \mathrm{ml}$. PK curve of twice daily administrated DRV/r performed at 35 weeks of gestation evidenced lower concentrations (30\%) in comparison to PP (C peak 7,610 ng/ml). Cord blood and amniotic fluid concentrations were $221 \pm 148 \mathrm{ng} / \mathrm{ml}$ and $466 \pm 374 \mathrm{ng} / \mathrm{ml}$, respectively. Mean gestational age at delivery was $37 \pm 1.3$ weeks and mean newborn weight was $2,940 \pm 367 \mathrm{~g}$. One case of pre term delivery (36 weeks) was evidenced. All children were HIV negative, no birth defects were reported.

Conclusions: Our data support efficacy, tolerability and safety of the 800/100 mg once and 600/100 mg twice daily administrated DRV/r for treatment in pregnancy, reporting DRV concentrations above the accepted minimum effective concentration $\left(\mathrm{EC}_{50}\right)$ for PI-resistant HIV-1 strains (MEC; $550 \mathrm{ng} / \mathrm{ml}$, based on in vitro studies). No general recommendation can be drawn from this small population $(n=5)$, but our observation suggests that DRV could be a valuable option in pregnancy despite altered pharmacokinetics usually reported for most of PI.

\section{PO 43}

FACTORS INFLUENCING THE ACCEPTANCE OF HIV TESTING IN PREGNANT WOMEN LIVING IN BISSAU

A. Riva* ${ }^{l}$, G. Erika ${ }^{l}$, F. Rankin Bravo ${ }^{2}$, D. Da Silva Te ${ }^{2}$, M. Acurie Aquessuen $^{2}$, A. Nanadje ${ }^{2}$,E. Mariani ${ }^{2}$,O. Bosisio ${ }^{2}, F$. Adorni $^{3}$, F. Croce $^{1}$, S. Rusconi ${ }^{4}$, M. Galli ${ }^{4}$

${ }^{I}$ III Divisione di Malattie Infettive, Ospedale L. Sacco, Milan, Italy; ${ }^{2}$ Associacao Ceu e Terras, Bissau, Italy; ${ }^{3}$ Institute of Biomedical Technologies, National Research Council, Segrate; ${ }^{4}$ Dipartimento di Scienze Cliniche, Sezione di Malattie Infettive e Immunopatologia, Università di Milano, Milan, Italy

Background: Stigma, socio-demographic, behavioral factors and the different strategies adopted for HIV testing and counselling (HTC) may influence the rate of test acceptance in Sub-Saharan Africa, rates have been frequently reported to be lower in West African Countries. Methods: All pregnant women attending the antenatal clinics in Bissau City, from January 2002 to May 2005, were asked to participate to an opt-out HTC program and, PMTCT was offered to HIV-1 positive women,. At the same time, a radio campaign focused on HIV/ AIDS awareness and prevention was, broadcasted all over the region. Results: 27,166 women were consecutively invited to join the HTC initiative. Of them, 369 (1.4\%) refused to be counseled and 2,928 $(10.8 \%)$ did not perform the HIV testing after being counseled. Test refusal accounted for $23 \%$ of women in 2002, but only for $6.4 \%$ in 2006 ( $p<0.001)$. Independent correlates of test denial resulted age (girls $<18$ years old at higher risk than women aged 18-34 years $(\mathrm{p}=0.005))$, illiteracy $(<0.001)$ and reporting only one sexual partner in the last 12 months $(\mathrm{p}<0.001)$.

Conclusions: The high acceptance rate of HIV testing in a WestAfrican setting confirms the effectiveness of the opt-out strategy if provided together with HIV care and prevention. The progressive decrease of test refusal rate likely derives from the educational campaign and the increasing availability of well-functioning testing centers funded by the project. However, the higher test refusal among illiterates and younger women reveals the existence of particularly disadvantaged groups needing targeted intervention.

\section{PO 44}

HYPOVITAMINOSIS D AND SECONDARY

HYPERPARATHYROIDISM IN HIV-INFECTED PATIENTS: A CASE-CONTROL STUDY

E. Pontali ${ }^{1}$, A. Giusti ${ }^{2}$, G. Penco ${ }^{1}$, R. Piscopo ${ }^{*}{ }^{1}$, M. Feasi ${ }^{1}$, A. Barone ${ }^{2}$, M. Razzano ${ }^{2}$, M. Pizzonia ${ }^{2}$, G. Cassola ${ }^{1}$, G. Pioli $^{3}$

${ }^{1}$ Department of Infectious Diseases, E.O. Ospedali Galliera, Genoa, Italy;

${ }^{2}$ Department of Geriatrics, E.O. Ospedali Galliera, Genoa, Italy; ${ }^{3}$ ASMN Hospital, Reggio Emilia 
Introduction: We explored the prevalence of vitamin D deficiency and secondary hyperparathyroidism (sHPTH) in a case-control study of $100 \mathrm{HIV}$-infected patients (HIV) and 100 matched controls.

Methods: HIV-cases were included if they were naïve to antiretroviral therapy (ART) or receiving first line ART. Healthy controls were matched for age (age range 25-65 years) and gender. Subjects, recruited between February-April 2009, underwent a standardized assessment to evaluate demographic and anthropometric characteristics, bone mineral density (BMD), serum calcium, phosphate, 25-hydroxy-vitamin D (25OHD), PTH, markers of bone turnover, clinical history and ART regimens (HIV only). The proportion of patients with $25 \mathrm{OHD}<20 \mathrm{ng} / \mathrm{ml}$ or $<30 \mathrm{ng} / \mathrm{ml}$, and PTH $>65 \mathrm{pg} / \mathrm{ml}$ were calculated. Secondary analysis were undertaken to evaluate variables associated with vitamin D deficiency in HIV.

Results: The two groups were comparable regard to baseline characteristics, except for alcohol intake, level of physical activity and prevalence of fractures. A significantly greater proportion $(\mathrm{p}<.001)$ of HIV had 25OHD levels $<20 \mathrm{ng} / \mathrm{ml}(75 \%)$ or $<30 \mathrm{ng} / \mathrm{ml}(88 \%)$ compared to controls ( 6 and $18 \%$ respectively). $19 \%$ of HIV and none of controls presented with sHPTH. The mean \pm standard deviation (SD) concentration of $25 \mathrm{OHD}$ was $16 \pm 11 \mathrm{ng} / \mathrm{ml}$ in HIV and $37 \pm 17 \mathrm{ng} / \mathrm{ml}$ in controls $(\mathrm{p}<.001)$. The corresponding figures for PTH were $47 \pm 23 \mathrm{pg} / \mathrm{ml}$ and $40 \pm 13 \mathrm{pg} / \mathrm{ml}(\mathrm{p}=.017)$, respectively. In HIV cases, 25OHD and PTH concentrations were not related to absolute CD4 T-cell count $(\mathrm{p}=.301)$, duration of HIV infection $(p=.753)$, co-infection of $\mathrm{HCV}(\mathrm{p}=.722)$ or ART $(\mathrm{p}=.655)$.

Conclusions. In HIV-infected adults hypovitaminosis D and sHPTH are highly prevalent and apparently not related to the clinical course of the disease. These results provide support for screening 25OHD and PTH in HIV-infected patients. Further research is warranted to establish the potential beneficial effects of vitamin D repletion in this population, since vitamin D has immunomodulatory effects.

\section{PO 45}

IMMUNO-VIROLOGIC FACTORS INVOLVED IN CARDIO VASCULAR DISEASE (CVD) RISK IN HIV-INFECTED, ARTTREATED INDIVIDUALS

S. Parisotto ${ }^{2}$, S. Passerini ${ }^{1}$, P. Meraviglia ${ }^{1}$, M. Schiavini ${ }^{3}$, F. Niero $^{l}$, P. Bonfanti ${ }^{1}$, D. Trabattoni ${ }^{2}$, G. Rizzardini ${ }^{1}$, M. Clerici $^{2}$, S. Piconi ${ }^{l}$ ${ }^{I}$ Infectious Disease Units, H. L. Sacco, Milan, Italy; ${ }^{2}$ Chair of Immunology, University of Milan, Milan, Italy; ${ }^{3}$ Endocrinologic Unit, H. L. Sacco, Milan, Italy

Background: HIV-infected patients undergoing ART have a greater burden of subclinical and clinical atherosclerotic disease compared to general population. Recent evidences suggest a direct role of HIV infection in the pathogenesis of cardio-vascular diseases (CVD). However, it is still unclear whether chronic inflammation, endothelial cell activation, and the activation of the peculiar cytokine networks characteristic of HIV infection are associated with the increased prevalence of atherosclerosis disease.

Objective: Aim of this study was to evaluate possible associations between inflammation, metabolic factors and endothelial alterations in HIV-infection.

Materials and methods: Sixty-eight HIV-infected patients (45 ARTtreated and 23 naive individuals) were enrolled in the study. Fifty \% of patients in both groups had a high cardiovascular risk (Framingham value $>20 \%$ ). Echo-Doppler (carotid intima media thickness, IMT), the expression of adhesion and activation molecules on circulating immune cells, plasmatic inflammatory and metabolic indexes, as well as plasmatic pro- and anti-inflammatory cytokines and chemokines and soluble adhesion molecules were cross-sectionally evaluated. A multivariate analysis (corrected for age, CD4 nadir, and the Framingham value) was used.
Results: As compared to naive patients, ART-treated individuals showed: (1) significantly increased IMT ( $\mathrm{p}=0.007)$; (2) reduced hCRP levels $(\mathrm{p}=0.017)$; (3) lower plasmatic IL6, TNFalpha, sICAM1, and sVCAM1 levels $(\mathrm{p}=0.0036 ; \mathrm{p}<0.001 ; \mathrm{p}=0.011$; $\mathrm{p}=0.028)$; 4) higher percentages of CD44-, CD11a-, CD62L- and CD49d-expressing CD4 T cells $(\mathrm{p}<0.001 ; \mathrm{p}=0.001 ; \mathrm{p}=0.006$; $\mathrm{p}=0.03) ; 5)$ lower percentages of CD86/CD14 cells $(\mathrm{p}=0.003)$. No significant differences were observed in TLR2- and TLR4-expressing CD14 cell and in plasma LPS levels.

Conclusions: ART plays a critical role in the pathogenesis of atherosclerotic plaques in HIV-infected patients. Classical inflammatory parameters (hCRP, IL-6, etc.) are nevertheless reduced in these patients, suggesting a non-inflammatory etiology of atherosclerosis in ART individuals. Atherosclerosis in HIV-infected patients may be due to direct toxic effects of ART.

\section{PO 46}

CARDIOVASCULAR RISK FACTORS AND CAROTID INTIMAMEDIA THICKNESS ARE CORRELATED TO LOWER NEUROCOGNITIVE PERFORMANCE IN HIV-INFECTED PATIENTS M. Fabbiani ${ }^{\prime 1}$, N. Ciccarelli ${ }^{1}$, M.C. Silveri ${ }^{1}$, M. Tana ${ }^{2}$, S. Farina ${ }^{1}$, E.

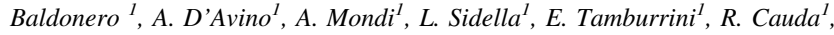
P. Grima ${ }^{2}$, S. Di Giambenedetto

${ }^{1}$ Catholic University of Sacred Heart, Rome, Italy; ${ }^{2}$ S. Caterina Novella Hospital, Galatina, Italy

Background: The relative role of metabolic and cardiovascular risk factors in the pathogenesis of HIV-associated neurocognitive disorders (HAND) is not fully understood.

Methods: We performed a cross-sectional study, consecutively enrolling asymptomatic HIV+ subjects during routine outpatient visits at two clinical centres. All patients underwent an extensive neuropsychological battery including 11 tests exploring memory, attention, executive abilities and fine motor skills. The Zung Depression Scale was also administered. At the time of neuropsychological examination carotid intima-media thickness (IMT) was also assessed. Cardiovascular risk factors were collected by patient interview and chart review. Factors associated with cognitive impairment were investigated by linear regression analysis.

Results: A total of 247 patients [75.3\% males, median age 46 years (IQR 39-52), 18.6\% with past AIDS-defining events, $84.2 \%$ with HIV-RNA $<50$ copies/mL, median current CD4 cell count $527 / \mu \mathrm{L}$ (IQR 388-717)] were enrolled. Overall, 93.9\% of subjects were on antiretroviral therapy (of whom $15.5 \%$ on abacavir-containing regimens), from median of 8 years (IQR 3.6-11.8). Among cardiovascular risk factors, the most reported were current smoking (53.8\%), dyslipidemia (25.1\%), hypertension (15\%) and diabetes $(6.1 \%)$. IMT was abnormal $(=0.9 \mathrm{~mm})$ in $32 \%$ of patients. The overall median of pathological (below the normative cut off) tests was 2 (IQR 1-4). At univariate analysis older age, diabetes, HCV-coinfection, past injecting drug use, previous exposure to protease inhibitors, a lower CD4 cell count nadir, an abnormal IMT and Zung depression score were associated to an higher number of pathological tests, while abacavir use and higher education resulted as protective factors. In multivariate analysis, only diabetes $(\mathrm{B}=1.27, \mathrm{p}=0.04)$, abacavir use $(B=-0.97, \quad p=0.013), \quad$ an abnormal IMT $(B=0.70$, $\mathrm{p}=0.041)$ and education $(\mathrm{B}=-0.23, \mathrm{p}<0.001)$ confirmed the association with the number of pathological task performances.

Conclusions: IMT and cardiovascular risk factors showed a strong association with lower cognitive performance, suggesting that metabolic factors could play a relevant role in the pathogenesis of HAND. Moreover, abacavir use was related to a better cognitive performance, possibly because of its good neuropenetration. The interplay of HIV, antiretroviral therapy and metabolic co-morbidities in the expression of HAND need to be further investigated. 
PO 47

DYSREGULATION OF ADIPOKINES IN HIV-INFECTED PATIENTS UNDER HAART IS A MARKERS FOR CARDIOVASCULAR RISK?

G. Ceccarelli* ${ }^{I}$, G. D'Ettorre ${ }^{I}$, S. Baroncelli ${ }^{2}$, C. Rizza ${ }^{I}$, F. Tierno ${ }^{I}$,

P. Massetti ${ }^{l}$, C.M. Mastroianni ${ }^{l}$, S. Vella ${ }^{2}$, V. Vullo ${ }^{I}$

${ }^{I}$ Department of Public Health and Infectious Diseases, University of Rome

"Sapienza"; ${ }^{2}$ Department of Drug Evaluation Italian Institute of Health ISS Rome

Background: Dysregulation of adipokines is implicated in the etiology of metabolic syndrome and related to increased cardiovascular risk. In fact altered levels of adipokines are strictly related with progressive atherosclerosis in HIV negative patients with diabetes, metabolic syndrome or obesity. We investigated the relationship between plasma adipokine levels and cardiovascular risk in HIV+ subjects without metabolic syndrome and with low cardiovascular risk.

Methods: We studied $40 \mathrm{HIV}+$ patients 30 on a first line stable and effective antiretroviral therapy and 10 HAART naive. 10 age-matched healthy HIV-patients were included as controls. Anthropometric, metabolic and immunovirologic parameters were measured in all patients. All HIV+ patients presented low cardiovascular risk $(<10 \%)$ according to the Framingham Scoring, and did not meet criteria for diagnosing metabolic syndrome. The levels of leptin and adiponectin were measured using commercially available ELISA. Coronary CTscan was performed in all patients.

Results: The mean age of patients was 42 years. CD4+ cell count was 396.3 cells $/ \mathrm{mmc}$ (mean) and HIV-RNA $<50$ copies $/ \mathrm{ml}$. The mean of metabolic and anthropometric parameters were normal in all HIV population. Coronary CT-scan did not evidence critical stenosis $(>50 \%)$ in coronary vessels of all HIV+ patients but showed an increase of medium degree of stenosis respect healthy population. Plasma adiponectin $(7.21 \mathrm{~g} / \mathrm{ml})$ and leptin $(23.1 \pm \mathrm{ng} / \mathrm{ml})$ levels in the group of HIV positive patients under HAART were lower than in HIV+ patients naive for antiretroviral therapy $(p=0.05)$. On the other hand we observed that plasma adiponectin and leptin levels in healthy control subjects are higher, with a statistical significance, than HIV+ patients under HAART but not than HIV subjects naive for antiretroviral therapy

Conclusions: Our data show that the adipokine levels are unexpected impaired in HIV+ patients under HAART without metabolic syndrome. Coronary risk of our cohort of HIV+ patients on antiretroviral therapy is low according to the Framingham Scoring, but dysregulation of adipokines observed predict an unexpected increase of cardiovascular risk, despite a normal metabolic profile. These data, although obtained in a small population, confirm that coronary risk is strongly underestimated by commonly used scores and indicate circulating adipokines levels as a possible marker for cardiovascular risk in HIV+ patients under HAART.

\section{PO 48}

ABACAVIR AND TENOFOVIR IMPACT ON ENDOTHELIAL

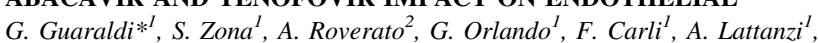
L. Manzini ${ }^{1}$, L. Bisi ${ }^{1}$, A. Cicognani ${ }^{1}$, R. Rossi ${ }^{1}$

${ }^{1}$ University of Modena and Reggio Emilia, Modena, Italy; ${ }^{2}$ University

of Bologna, Bologna, Italy

Background: Flow Mediated Dilation (FMD) of the brachial artery is an endothelial function test that has been used to assess the impact of cardiovascular induced drug toxicity. The objective of the study was to analyse the impact of abacavir and tenofovir on endothelial function.

Methods: Retrospective observational study of HIV infected patients with suppressed HIV viral load "switching in" or "switching out" either abacavir (ABC) or tenofovir (TDF) in the antiretroviral regimen. Endothelial function was assessed with FMD using a standard protocol. A sub-analysis was performed in the group of patients switching from $\mathrm{ABC}$ to TDF and from TDF to ABC. Differences between means were tested using the Student $t$ test for either paired or unpaired samples. Simple and multivariate linear regression analysis was used to investigate the linear association between variables. Sex, LDL-c, HDL-c, HOMA-IR, MDRD e-GFR value and nadir CD4 were used as covariates.

Results: Twenty patients were included in the $\mathrm{ABC}$, and 30 in the TDF, "switching in" and "switching out" protocol. Table 1 describes FMD change (delta FMD) in "switch in/out" $\mathrm{ABC}$ or TDF and the correlation between delta FMD and current drug treatment or time without drug.

\begin{tabular}{|c|c|c|c|c|c|c|c|}
\hline & $\begin{array}{l}\text { FMD } \\
\text { pre (\%) }\end{array}$ & $\begin{array}{l}\text { FMD } \\
\text { post }(\%)\end{array}$ & $\begin{array}{l}\text { Delta } \\
\text { FMD } \\
(\%)\end{array}$ & $\mathrm{p}$ value & $\begin{array}{l}\text { Time between } \\
\text { FMD } \\
\text { measurements }\end{array}$ & $\begin{array}{l}\text { Correlation } \\
\text { between } \\
\text { variables }\end{array}$ & $\begin{array}{l}\mathrm{p} \\
\text { value }\end{array}$ \\
\hline $\begin{array}{c}\text { "Switch in" } \\
\mathrm{ABC}\end{array}$ & 8.06 & & & & $( \pm 4.7)$ & $9.32( \pm 4.7)$ & 1.26 \\
\hline$( \pm 5.6)$ & 0.32 & 3.22 & & & $( \pm 1.0)$ & $\begin{array}{l}\mathrm{ABC} \text { exp. } \propto \Delta \mathrm{FMD} \\
\beta=-0.05\end{array}$ & 0.8 \\
\hline $\begin{array}{l}\text { "Switch out" } \\
\text { ABC }\end{array}$ & 9.75 & & & & $( \pm 7.0)$ & $11.12( \pm 6.2)$ & 1.37 \\
\hline$( \pm 7.4)$ & 0.42 & 2.49 & & & $( \pm 1.1)$ & $\begin{array}{l}\text { Time without } \mathrm{ABC} \\
\quad \propto \Delta \mathrm{FMD} \\
\beta=-0.02\end{array}$ & 0.92 \\
\hline $\begin{array}{l}\text { "Switch in" } \\
\text { TDF }\end{array}$ & 8.87 & & & & $( \pm 6.7)$ & $12.75( \pm 6.5)$ & 4.69 \\
\hline$( \pm 6.7)$ & $<0.001$ & 2.81 & & & $( \pm 0.93)$ & $\begin{array}{l}\text { TDF exp. } \propto \Delta \text { FMD } \\
\beta=0.16\end{array}$ & 41 \\
\hline $\begin{array}{l}\text { "Switch out" } \\
\text { TDF }\end{array}$ & 7.62 & & & & $( \pm 6.7)$ & $9.25( \pm 4.1)$ & 1.63 \\
\hline$( \pm 6.2)$ & 0.16 & 3.03 & & & $( \pm 1.2)$ & $\begin{array}{l}\text { Time without TDF } \\
\quad \propto \Delta \mathrm{FMD} \\
\beta=-0.40\end{array}$ & 0.029 \\
\hline
\end{tabular}

The difference between delta FMD in "switch in" versus "switch out" $\mathrm{ABC}$ was $0.46 \%$ (95\% CI $-4.8,3.9), \mathrm{p}=0.82$

The difference between delta FMD in "switch in" versus "switch out" TDF was $3.07 \%$ (95\% CI $-6.4,0.3), \mathrm{p}=0.07$

Table 2 Describes FMD change in patients switching from TDF to $\mathrm{ABC}$ and from $\mathrm{ABC}$ to $\mathrm{TDF}$

\begin{tabular}{clllll}
\hline & $\begin{array}{l}\text { No. } \\
\text { of } \\
\text { pts }\end{array}$ & $\begin{array}{l}\text { FMD } \\
\text { pre }\end{array}$ & $\begin{array}{l}\text { FMD } \\
\text { post }\end{array}$ & $\begin{array}{l}\text { FMD } \\
\text { p } \\
\text { value }\end{array}$ & $\begin{array}{l}\text { Time between } \\
\text { FMD } \\
\text { measurements }\end{array}$ \\
\hline $\begin{array}{l}\text { Switch TDF to } \\
\text { ABC }\end{array}$ & 11 & 8.44 & & $( \pm 5.3)$ \\
$9.87( \pm 4.89)$ & 1.48 & & & $( \pm 4.8)$ \\
0.32 & 3.19 & & & $( \pm 0.97)$ \\
Switch ABC & 7 & 8.80 & & $( \pm 8.5)$ \\
to TDF & & & & $( \pm 5.6)$ \\
$12.1( \pm 7.25)$ & 2.79 & & & $( \pm 0.96)$ \\
0.23 & 2.71 & & & & \\
\hline
\end{tabular}

HDL-c increase (delta HDL-c) was the only independent predictor of FMD increase in patients from $\mathrm{ABC}$ to TDF $(\beta=0.21, \mathrm{p}<0.01)$ 
Conclusions: $\mathrm{ABC}$ appears to have a neutral effect on endothelial function, TDF appears to increase FMD value in association with a favorable lipid profile change.

\section{PO 49}

PLASMA CONCENTRATIONS OF SOLUBLE ENDOTHELIAL PROTEIN C RECEPTOR AND LIPID METABOLISM CHANGE WITH HIV-RNA AND CD4 CELL COUNTS IN MULTI-EXPERIENCED HIV1+ PATIENTS TREATED WITH MARAVIROC-BASED ART

S. Nozza ${ }^{1}$, E.M. Faioni ${ }^{2}$, M. Pogliaghi ${ }^{1}$, G. Fontana ${ }^{2}$, C. Razzari ${ }^{2}$,

S. Chiappetta ${ }^{I}$, L. Galli ${ }^{I}$, V. Spagnuolo ${ }^{1}$, G. Tambussi ${ }^{1}$

${ }^{I}$ San Raffaele Scientific Institute, Milan, Italy; ${ }^{2}$ Università degli Studi di

Milano and Az. Ospedaliera San Paolo, Medicina III, Dipartimento di

Medicina, Chirurgia e Odontoiatria, Milan, Italy

Background: HIV infection is associated with disimmunity, chronic inflammation and coagulation activation. The endothelial protein $\mathrm{C}$ receptor (EPCR) participates in the activation of protein $\mathrm{C}$, an important regulator of coagulation and inflammation and possesses itself anti-inflammatory activities; its soluble form is shed from the endothelium and found in plasma. Maraviroc (MVC) is a CCR5 antagonist with anti-inflammatory properties. Aim of the study was to evaluate changes of coagulation and inflammation markers in chronically infected multi-experienced HIV+ patients, treated or not with MVC-based antiretroviral therapy (ART).

Methods: $88 \mathrm{HIV}+$ patients (70 men, 18 women) treated with MVCbased $(n=45)$ or PI-based therapy $(n=43)$ were evaluated at baseline, 4, 8, 12, 24, 36 and 48 weeks. D-dimer, sEPCR, soluble thrombomodulin (sTM), interleukin-6 (IL-6) and hsCRP were measured as well as metabolic variables.

Results: At baseline MVC treated and not treated groups differed only for triglycerides levels (median level $134 \mathrm{mg} / \mathrm{dL}$ vs. 214, $\mathrm{p}<0.01$ ). Such difference was observed also at week 4 and week 12 , after which the non MVC treated group reached similar levels to the treated one. Similarly, HDL-cholesterol was higher in the MVC treated group at weeks 4 and 12 (week 4: median $44 \mathrm{mg} / \mathrm{dL}$ vs. 35 and week 12 : 48 vs. $35, \mathrm{p}<0.01$ and $<0.05)$. CD4+ cell counts were significantly higher in the MVC treated group starting from week 4 and up to week 48, and so were CD4/CD8 ratios and CD4 percentages starting from week $12(\mathrm{p}<0.05)$. Soluble EPCR $(\mathrm{sEPCR})$ increased over time in both treatment groups, though not significantly. Finally, in MVC treated patients, absolute changes of sEPCR levels were positively correlated with changes in CD4+ cell counts (Spearman rho $=0.476, \mathrm{p}<0.01)$ and negatively correlated with changes in HIV-RNA (Spearman $\rho=-0.429, \mathrm{p}<0.01$ ), while in non MVC treated patients a negative correlation was observed only between absolute changes of sEPCR levels and changes in CD4+ cell counts (Spearman $\rho=-0.377, \mathrm{p}<0.05$ ).

Discussion: The use of Maraviroc in this population of multi-experienced HIV-1+ patients seems to positively affect the lipid profile of the studied population. Moreover, CD4 cell gain is much more pronounced and rapid in those patients treated with MVC-based ART. Interestingly, the increase of sEPCR, as marker of endothelial integrity, could be a marker of the rapid immune reconstitution observed in the MVC group.

\section{PO 50}

MENOPAUSE IN HIV INFECTED PATIENTS: WOMEN

IN A MIDLIFE POLYPATHOLOGY CRISIS

C. Berardi ${ }^{I}$, R. Rossi ${ }^{I}$, S. Zona ${ }^{I}$, G. Orlando ${ }^{I}$, F. Carli ${ }^{I}$, C. Casalgrandi ${ }^{I}$,

A. Bedini ${ }^{l}$, S. Cocchi ${ }^{l}$, M.G. Modena ${ }^{1}$, G. Guaraldi ${ }^{l}$

${ }^{I}$ University of Modena and Reggio Emilia, Modena, Italy

Background: Menopause can be considered a paradigm of physiological ageing. The aim of this study was to compare the physical and psychological health profile of postmenopausal women with and without HIV. Secondary objective was to assess polypathology prevalence and risk factors as a surrogate of ageing phenotype.

Methods: Cross-sectional case-control study of consecutive menopausal HIV infected women experienced to ART were recruited from a third level University Hospital. Age and race matched controls were randomly selected among patients between 45 and 60 years old assisted by a family medical practitioner. Physical health assessment included prevalence of single co-morbidities (osteoporosis, renal failure, hypothyroidism, diabetes) and Polypathology (Pp, defined as the presence of 2 or more co-morbidities in the same individual). Subclinical atherosclerosis was assessed by Pulse Wave velocity (PWV) and Intima Media thickness (IMT) of common and internal carotid. Osteoporotic fracture risk was assessed by FRAX score. Psychological health assessment explored depression with CESD-10 scale, quality of life in menopause with MEN-QoL and sexual dysfunction with FSFI questionnaire. Mann-Whitney $U$ and Fisher exact test were used to compare continuous and categorical variables in cases and controls. Logistic regression analysis was performed to evaluate the probability of presence of Pp in groups. Multivariable linear regression analyses was used to compare age adjusted PWV data.

Results: Age at menopause was younger in cases $(47 \pm 6$ vs. $50 \pm 6$, $\mathrm{p}=0.01$ ) after correcting for calendar age, race, BMI and smoking. A trend to higher prevalence of any co-morbidities and $\mathrm{Pp}$ was found in cases compared to controls. Time from menopause (years) was associated to a trend of increased risk for $\mathrm{Pp}$ in cases compared to controls $(\mathrm{p}=0.11)$ (Fig. 1).

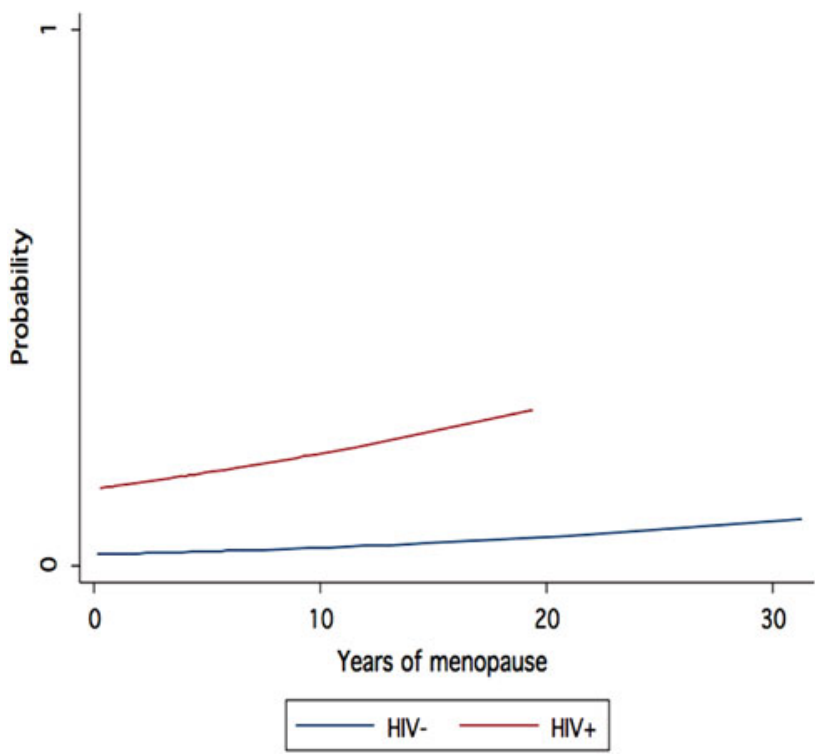

HIV infected women showed higher FRAX score $(\mathrm{p}<0.001)$ and age-adjusted PWV ( $\mathrm{p}=.007)$, but not higher carotid IMT.

A significant lower psychological health profile was found in cases compared to controls regarding depression symptoms $(\mathrm{p}=.005)$, sexual dysfunction $(\mathrm{p}=.02)$ and physical menopausal symptoms $(\mathrm{p}=.050)$.

Conclusions: Ageing phenotype in HIV infected women with menopause is characterized by a poorer physical and psychological health profile compared to HIV negative controls. Early onset of menopause and a trend towards a higher impact of years of menopause in Pp risk in cases compared to controls suggest that HIV may play a role in accelerating natural ageing process. 
PO 51

THE SENSE TRIAL: ETRAVIRINE SHOWS LOWER PREVALENCE AND SEVERITY OF NEUROPSYCHIATRIC ADVERSE EVENTS COMPARED TO EFAVIRENZ IN TREATMENT-NAÏVE PATIENTS

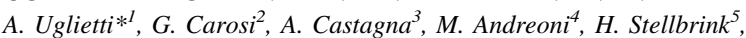

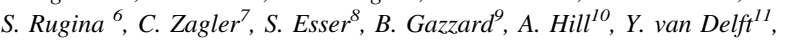

S. Marks ${ }^{11}$

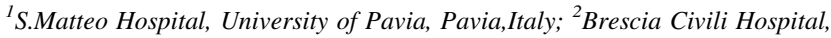
University of Brescia, Brescia, Italy; ${ }^{3}$ Fondazione San Raffaele, Milan, Italy; ${ }^{4}$ Tor Vergata Hospital, University of Rome, Rome, Italy;

${ }^{5}$ Infektionsmedizinisches Centrum, Hamburg, Germany; ${ }^{6}$ Spitalul Clinic de Boli Infectioase, Constanta, Romania; ${ }^{7}$ SMZ Baumgartner, Vienna, Austria, Vienna; ${ }^{8}$ Universitätsklinikum, Essen, Germany; ${ }^{9}$ St Stephens Centre, Chlesea and Westminster Hospital, London, UK, ${ }^{10}$ Liverpool University and Tibotec BVBA, Liverpool, UK; ${ }^{11}$ Janssen-Cilag, Tilburg, The Netherlands

Background: Efavirenz (EFV) treatment is associated with a range of neuropsychiatric (NPS) adverse events (AEs), which differ in duration and severity.

Methods: In this double-blind placebo-controlled trial, 157 treatmentnaïve patients with HIV RNA $>5,000$ copies $/ \mathrm{mL}$, were randomised 1:1 to either etravirine (ETR) $400 \mathrm{mg}$ once daily $(\mathrm{n}=79)$, or EFV $600 \mathrm{mg}$ once daily $(\mathrm{n}=78)$, plus two NRTIs. After 12 weeks of randomised treatment, the type and frequency of NPS AEs was compared between treatment arms.

Results: Overall, the patients were $81 \%$ male, $85 \%$ Caucasian, with a median age of 36 years. Median baseline CD4 Count was 302 cells/ $\mu \mathrm{L}$, median HIV RNA $4.8 \log _{10}$ copies/mL. In the primary analysis, $13 / 79$ patients $(16.5 \%)$ in the ETR arm, versus $36 / 78(46.2 \%)$ in the EFV arm, showed at least one Grade 1-4 treatment-emergent drugrelated NPS AE $(\mathrm{p}<0.001)$. The most common neurological AE was dizziness, reported for 3 patients in the ETR arm versus 15 in the EFV arm. The most common psychiatric adverse events were sleep disorders, reported in 7 patients in the ETR arm versus 25 patients in the EFV arm. The prevalence of Grade 1-4 all cause NPS AEs showed a peak at Week $2(21.5 \%$ in the ETR arm and $43.6 \%$ in the EFV arm), but at the Week 12 visit, the percentage with an ongoing Grade 1-4 all cause NPS AE remained different between the arms (21.7\% with ETR and $35.7 \%$ with EFV). In the ETR arm, 29 all cause NPS adverse events were reported: 20 Grade 1,7 Grade 2 and 2 Grade 3 . In the EFV arm, 93 NPS adverse events were reported: 55 Grade 1, 34 Grade 2 and four Grade 3. New medication for NPS adverse events was started for $7.6 \%$ of patients in the ETR arm versus $16.7 \%$ of patients in the EFV arm. One patient in the ETR arm and five in the EFV arm discontinued randomized treatment with NPS AE's.

Conclusions: In the SENSE trial, first-line treatment with ETR $400 \mathrm{mg}$ once daily + 2NRTIs led to significantly fewer NPS AEs, compared with EFV + 2NRTIs. These NPS AEs were mainly Grade 1 or 2 in severity. The difference between the arms emerged at Week 2, but persisted through Week 12 .

\section{PO 52}

\section{THE EFFECTS OF LIPOIC ACID EYE DROPS IN HIV-POSITIVE} PATIENTS

A. Uglietti ${ }^{*}$, S. Pezzotta ${ }^{2}$, E. Antoniazzi ${ }^{2}$, R. Maserati ${ }^{1}$

${ }^{I}$ Clinica Malattie Infettive-Fondazione IRCCS Policlinico San Matteo, Pavia, Italy; ${ }^{2}$ Clinica Oculistica, Fondazione IRCCS Policlinico San Matteo, Pavia, Italy

Background: Decreased tear production has been reported in $<1.0 \%$ of the general population but in $20-39 \%$ in HIV-positive individuals. The incidence of the dry-eye syndrome (DES) under HAART remains unchanged compared to the incidence in the pre-HAART era. DES, likely caused either by HIV itself and HAART, is a tear film alteration involving both lipid and mucous lacrimal layers. Usually DES is treated with tear drops (ipromellosa or ialuronic acid) to avoid further corneal alterations. A new compound, lipoic acid, featured with antioxidant, epitheliotropic and neurotropic characteristics was evaluated in a eye drop association with ipromellosa in HIV-positive patients.

Methods: This study (prospective, randomized, controlled, single blinded) assessed the effect of ipromellosa plus lipoic acid (group A) versus ipromellosa only (group B) eye drops on DES in HIV-positive patients. Patients were enrolled if complaining any eye drynessassociated symptom (burning, itching, foreign body sensation, photophobia) and hyperaemia. We excluded patients with infectious kerato-conjunctivitis, ocular inflammation, erosions and corneal ulcers, severe systemic comorbidities or previous surgery in anterior or posterior segment in the last 6 months. One drop of the randomized preparation was given in each eye three times a day for 3 months. The evaluation consisted in: physical examination, biomicroscopic exam, visual acuity, Schirmer test and tear break-up time test (BUT) measured in both eyes. Questionnaire about dry-eye symptoms and quantitative tests have been performed in baseline visit, then at 15, 30 and 90 days.

Results: Eighteen patients (36 eyes) were randomized but only 32 eyes were analyzed. The demographic characteristics were similar in two groups. Mean age was 43 years (range 28-56), males were 11. All patients were on combination antiretroviral therapy (cART), HIVRNA was undetectable and median CD4 cell counts was 421 cells $/ \mu 1$ (range 133-720). BUT improved significantly in group A versus $\mathrm{B}$ (at baseline: $5 \mathrm{~s} \pm 2$ and $6.17 \mathrm{~s} \pm 1.60$; after 90 days: $10.2 \mathrm{~s} \pm 1.48$ and $7 \mathrm{~s} \pm 1.9$, respectively, $\mathrm{p}=0.01)$. Photophobia and hyperaemia also improved in a significant way $(\mathrm{p}=0.03$ for both parameters) in the group A versus B at the 3-month evaluation. A significant trend favouring group A was noted for other symptoms. The immune status as measured by CD4+ cell count and percentage did not changed during the observational period.

Discussion: Patients treated with lipoic acid-containing eye drops showed a significant improvement in their inflammatory process when compared to a control treatment. This may be due to a quick stabilization of lacrimal film (already present at 15 days), irrespective of immune-virological status. Although the number of patients was limited, our results confirmed the epithelio-neuroprotective activity and epithelio-neurotropic, anti-oxidant functions of lipoic acid.

PO 53

EVALUATION OF ENDOTHELIAL FUNCTION AND ACTIVATION MARKERS IN HIV-1 INFECTED PATIENTS ON SUPPRESSIVE HAART AFTER SWITCHING TO ABACAVIR/LAMIVUDINE FIXED DOSE: PRELIMINARY RESULTS

A. Esposito $*^{1}$, D. Pastori ${ }^{2}$, R. Carnevale ${ }^{2}$, L. Perri ${ }^{2}$, C. Fimiani ${ }^{1}$, M. Schillizzi ${ }^{2}$, C. Calabrese ${ }^{2}$, A. Fantauzzi ${ }^{I}$, S. Basili ${ }^{2}$, I. Mezzaroma ${ }^{1}$

${ }^{I}$ Dipartimento di Medicina Clinica, "Sapienza", Università di Roma, Rome, Italy; ${ }^{2}$ Dipartimento di Medicina Interna e Specialitá Mediche, "Sapienza", Università di Roma, Rome, Italy

Background: Increasing evidences suggest that HIV-1+ subjects have a significant raise in cardiovascular risk [1-3]. A key role is played by HIV-1, immune activation and chronic inflammation. Furthermore, alterations in lipid and glucose metabolism associated with antiretrovirals are described [4-6]. The DAD Study showed a significant increase of myocardial infarction risk in patients on $\mathrm{ABC}$, characterized by a rapid occurrence and not linked to the treatment length [7]. Biological mechanisms are still unclear. Clarify these points would allow a more accurate selection of patients to be treated with ABC, as demonstrated by HLA-B5701 screening [8].

Objectives: To determine the role of $\mathrm{ABC} / 3 \mathrm{TC}$ on vascular homeostasis and cardiovascular risk compared to ZDV/3TC or TDF/FTC. Evaluated parameters (baseline and every 3 months): echocardiography, ABI, IMT, FMD, ROS, PAC-1, glucose, total cholesterol, LDL and HDL, triglycerides, ESR, CRP, fibrinogen and D-dimers, HLADR and CD38 on CD4+ and CD8+ T lymphocyte. Study design: 40 
patients (20 treated with TDF/EMT and 20 with ZDV/3TC) enrolled; 10 per arm switched their NRTI fixed dose to ABC/3TC.

Results: 15 patients on TDF/FTC (Group 1) and 15 on ZDV/3TC (Group 2) were enrolled. 10 per group reached 3 months. At baseline both groups showed homogeneous demographic characteristics. No differences were present in cardiovascular and metabolic parameters. Activation and inflammatory markers, oxidative stress and platelet activation were similar. Only CRP showed a significant difference $(2,810 \mathrm{~g} / \mathrm{l}$ in Group 1 vs. $1,190 \mathrm{~g} / 1$ in Group 2). After 3 months Group 1 showed no differences in all evaluated parameters In Group 2, ABI had a significant change, rising from values of potential pathological status to normal and FMD values improved. All other parameters remained unchanged. Both groups showed an increase in total cholesterol and LDL. No changes in CD4+ T cells occurred, whereas a decrease of activation markers on CD4+ $\mathrm{T}$ cells was present in both groups.

Conclusions: These results indicate some preliminary observations. (1) The ABC pro-inflammatory effect seems unconfirmed in the short term. (2) FMD tends to increase after the switch in both groups, suggesting an anti-atherogenesis role of $\mathrm{ABC} / 3 \mathrm{TC}$; also IMT tends to decrease in both groups, whereas ABI shows a significant improvement after switch only in ZDV/3TC patients. The data, needing to be confirmed, indicate a positive effect on vascular homeostasis after switching to $\mathrm{ABC} / 3 \mathrm{TC}$ also in subjects previously on TDF/FTC.

\section{PO 54}

\section{EFFICACY AND SAFETY OF TENOFOVIR DISOPROXIL} FUMARATE IN A SINGLE CENTER COHORT

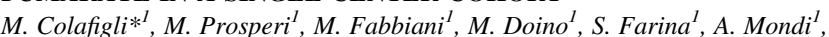
A. D'Avino ${ }^{I}$, I. Fanti ${ }^{I}$, R. Cauda ${ }^{I}$, S. Di Giambenedetto ${ }^{I}$

Università Cattolica del Sacro Cuore, Rome, Italy

Background: Tenofovir disoproxil fumarate (TDF) is a first-choice nucleotide reverse transcriptase inhibitor (NtRTI) according to the major guidelines. We evaluated its safety and efficacy in the long term in a real-life setting.

Methods: Observational, single center study. Patients starting TDF were included in the analysis if they had at least a follow-up visit. Clinical and laboratory data were collected from the clinical records. Follow-up was censored at the discontinuation of TDF, death or loss to follow-up or at the last visit in our Outpatient service. Changes in backbones did not influence the duration of TDF-containing regimens. Time and predictors of discontinuation of TDF was evaluated using Kaplan-Meier and Cox's analyses. After preliminary univariable screening of $>110$ variables, a final multivariable model (40 variables) was produced.

Results: 1,492 patients (19.1\% naïve, $20,1 \%$ second line and $60.7 \%$ later lines of therapy) starting TDF from 2002 to 2010 were included in the analysis for a median duration of the TDF-containing therapy of 21.3 weeks (IQR 9.3-37.6) and a 4,812.9 person-year follow-up (PYFU). 414 discontinuation events occurred (incidence: 8.6 per 100 PYFU); the probability to continue the TDF-based therapy was 0.898 $(0.882-0.914)$ for the first year, $0.833(0.813-0.853)$ at 2 years and $0.652(0.622-0.683)$ at 5 years. $9.1 \%$ of patients discontinued TDF for virological failure (VF), $40 \%$ for toxicity, $13.6 \%$ for inadequate adherence, $18.3 \%$ for simplification, $7.9 \%$ for other reasons and in $11 \%$ of cases the reason was unknown. TDF was discontinued in $16 / 89(18 \%)$ naïve patients, $38 / 203(16.1 \%)$ experienced patients with baseline VL $<50 \mathrm{c} / \mathrm{mL}$ and 284/936 (30.3\%) experienced patients with baseline VL $>50 \mathrm{c} / \mathrm{mL}$. Among experienced patients with baseline $\mathrm{VL}<50 \mathrm{c} / \mathrm{mL}$ the $\mathrm{VF}$ rate was $5.3 \%$ (2 events) and the toxicity rate was $42.1 \%$ (16 events) whereas among experienced patients with baseline $\mathrm{VL}>50 \mathrm{c} / \mathrm{mL}$ the $\mathrm{VF}$ were $9.7 \%$ (28 events) and the toxicity rate was $37.7 \%$ (109 events). More recent calendar year in which TDF was started, higher baseline creatinine and concomitant use of DDI independently predicted the interruption of the
TDF-containing regimen at multivariate analysis in the whole population whereas higher CD4 cell count at last follow-up and ritonavirboosted protease inhibitors or entry/integrase inhibitors at treatment interruption negatively predicted the outcome. Median creatinine at last follow-up visit was $1 \mathrm{mg} / \mathrm{dL}$ (IQR); 210/1,375 (15.3\%) had a creatinine higher than $1.3 \mathrm{mg} / \mathrm{dL}$ at treatment interruption.

Conclusions: In our Clinic's real-life practice, TDF was safe, efficacious and well tolerated. Discontinuations as well as high creatinine levels leading to treatment discontinuation were infrequent.

\section{PO 55}

BONE MINERAL DENSITY (BMD) FOLLOW UP AMONG HIVINFECTED SUBJECTS: PREDICTORS OF LOW BMD AT BASELINE M. Feasi ${ }^{* 1}$, G. Penco ${ }^{1}$, A. Giusti ${ }^{2}$, M. Pizzonia ${ }^{2}$, M. Razzano ${ }^{2}$, A. Barone ${ }^{2}$, R.

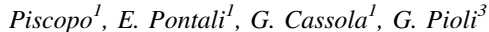

${ }^{I}$ Department of Infectious Diseases, E.O. Ospedali Galliera, Genoa, Italy; ${ }^{2}$ Department of Geriatrics, E.O. Ospedali Galliera, Genoa, Italy; ${ }^{3}$ ASMN Hospital, Reggio Emilia, Italy

Background: To assess the prevalence of low bone mineral density (BMD) at baseline and to identify predictors for osteopenia/osteoporosis in a prospective monocentric cohort of north-Italian HIV+ patients.

Methods: The cohort included only subjects who were naive to antiretroviral therapy (ART) or receiving their first ART. Subjects underwent a standardized baseline assessment to evaluate demographic and anthropometric characteristics, clinical history, biochemical parameters (including CD4 count and HIV viral load), ART regimen (if any). Lumbar spine, femoral neck and total hip BMD measurements were obtained by DXA. $\mathrm{T}$ scores and $\mathrm{Z}$ scores were recalculated using the appropriate NHANES reference values. Low BMD was defined by a $T$ score $<-1$ in at least one of the three sites considered. To evaluate predictors of low BMD bivariate and multivariate analyses were performed. First, it was assessed the bivariate association between low BMD and variables collected at baseline. Then, all co-variates associated with low BMD with a $p<0.1$ were retained and included in a logistic regression analysis.

Results: The prospective cohort included $112 \mathrm{HIV}+$ subjects (males $=68$, females $=44$ ), aged 25-69 years were enrolled in the study. Overall $12 \%$ of patients presented osteoporosis (T-score $<-2.5$ ) and $35 \%$ presented osteopenia (T-score $<-1>-2.5$ ). Univariate analysis showed the following significant associations with low BMD: older age $(\mathrm{p}=0.003)$; current smoking $(\mathrm{p}=0.024)$, reduced creatinine clearance $(\mathrm{p}=0.019)$; increased interleukin-6 $(\mathrm{p}=0.047)$. Low calcium intake $(p=0.08)$ and current use of protease inhibitors $(p=0.059)$ were associated with low BMD at the limit of significativity. Interestingly, in a multivariate model, only reduced creatinine clearance (OR $2.55,95 \% \mathrm{CI}$ $1.07-6.08, \mathrm{p}=0.034)$ and current smoking (OR 3.04, 95\% CI 1.24-7.44, $\mathrm{p}=0.015$ ) still resulted significantly associated to low BMD.

Conclusions: In our population the prevalence of osteopenia and osteoporosis was similar to those previously reported. Interestingly, nor ART, neither tenofovir use were associated with low BMD. Actually, the only identified predictors of low BMD at baseline (reduced renal function and current smoking) are well acknowledged as typical risk factors for osteoporosis and/or bone diseases. Prospective data will help in verifying their importance in the next stages of HIV infection or in the next phases of ART in this cohort.

\section{PO 56}

VITAMIN D AND CARDIOVASCULAR RISK IN HIV+ PATIENTS

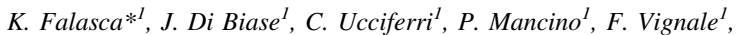

M. Vannucci ${ }^{l}$, E. Pizzigallo ${ }^{l}, J$. Vecchiet ${ }^{l}$

${ }^{I}$ Clinical Infectious Diseases, "G. d'Annunzio" University Chieti-Pescara, Chieti, Italy

Introduction: There are several studies showing a strong association between vitamin D deficiency (vit D) and cardiovascular risk (CVR) 
in the general population. The studies about the lack of vitamin D in the course of HIV infection are few. The aim of this study was to analyze the association between vit D and CVR in HIV+ patients.

Materials and methods: We enrolled a total of $100 \mathrm{HIV}+$ patients, who were divided according to the RVC calculated by the Framingham Risk Score. We analyzed the following parameters: systolic blood pressure (SBP) and diastolic (DBP), triglycerides (TG), total cholesterol (ColTot), HDL, LDL, glucose, HOMA index, BMI, CRP, ESR, microalbuminuria, cystatin C, Na, K, Ca, Vit D.

Results: 20 patients (mean age $52 \pm 11$ years) with high CVR (>10\%) showed statistically lower levels of serum vitamin D compared to 90 patients with low CVR $(=10 \%) \quad(13 \pm 11$ versus $20 \pm 12 \mathrm{mg} / \mathrm{dl}, \mathrm{P}<0.01)$. Patients with high CVR showed statistically higher levels of SBP, TG, ColTot and blood glucose and lower HDL levels than patients with low CVR. In addition, multiple regression analysis showed that each decrease of $10 \mathrm{mg} / \mathrm{dl}$ of vitamin D has a $85 \%$ increase in CVR (OR 1.85, 95\% CI 1.07-3.76, $\mathrm{P}<0.04$ ), irrespective of disease duration and the number of CD4 cells.

Conclusions: It is known that levels of vitamin D in the general population are inversely correlated with coronary artery disease, associated with hypertension, diabetes mellitus and dyslipidemia. Recently it was shown that vitamin D has effects on endothelial function and reduces vascular calcification. In addition, the calcification of the coronary arteries is inversely correlated with levels of vitamin D. The potential mechanisms underlying this association appears to be various, due to either an increased arterial calcification, impaired endothelial function, or a direct effect on cardiac contractility that dysregulates inflammatory cytokines. Our study shows that in HIV+ patients the vit D deficiency is associated with an increased CVR. This deficit depends on the duration of the infection, but not on the presence of the HIV virus itself.

\section{PO 57}

LONG-TERM SAFETY OF A DUAL REGIMEN OF RALTEGRAVIR AND RITONAVIR-BOOSTED PROTEASE INHIBITOR IN ARVEXPERIENCED PATIENTS WITH CHRONIC KIDNEY DISEASE D. Segala* ${ }^{\prime}$, R. Roda ${ }^{I}$, M. Libanore $^{I}$, L. Sighinolfi ${ }^{l}$

${ }^{1}$ U.O.Malattie Infettive, Ferrara, Italy

Objective: to evaluate the long-term safety of a dual regimen of raltegravir (RAL) and ritonavir-boosted protease inhibitor $(\mathrm{PI} / \mathrm{r})$ in ARV experienced patients with chronic kidney disease (CKD)

Methods: prospective, single centre, study in patients on treatment with $2 \mathrm{NRTI}+\mathrm{PI} / \mathrm{r}$ who switched to RAL and PI/r because of CKD. Demographic, epidemiological, laboratory, viro-immunological data were collected using medical report database. Patients were required to have undetectable viral load at baseline and during the previous 12 months. CKD was defined on the basis of National Kidney Foundation Guidelines (2002).

Results: 8 patients were included: $7 / 1$ male, median age 50 years. At baseline all patients had HIV RNA $<40$ copies/ml and median CD4 540 cells/mmc. 4/8 were receiving TDF/FTC as backbone and 4/8 ABC/3TC; $3 / 8$ showed severe CKD (stage 4), and 5/8 moderate CKD (stage 3). Patients switched from 2NRTI to RAL maintaining the same PI/r (5 LPV/r, 2 DRV/r, 1 ATZ/r). After a median follow-up of 19 months all patients were on treatment. At the latest visit, median CD4 was $661 / \mathrm{mmc}$, HIV RNA was below 40 copies $/ \mathrm{ml}$ in all patients. 4/8 patients had a mild CKD (Stage 2), 3/8 moderate CKD (stage 3) and 1/8 maintained severe CKD (stage 4). No other clinical or laboratory adverse events were observed.

Conclusion: Our data suggest that dual regimen of RAL and PI/r could be an attractive and safe options in patients with chronic kidney disease. Limitation of this study is the low number of patients included. Further randomized clinical trials are needed in order to confirm the efficacy and safety of this strategy.
PO 58

PREVALENCE AND INCIDENCE OF MAJOR CARDIOVASCULAR EVENTS IN HIV-INFECTED PATIENTS: A PROSPECTIVE COHORT STUDY

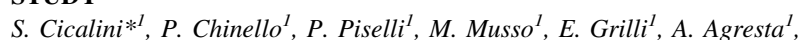
N. Petrosillo

${ }^{I}$ National Institute for Infectious Diseases Lazzaro Spallanzani, IRCCS, Rome, Italy

Objective: To determine the cardiovascular $(\mathrm{CV})$ risk profile, and to evaluate the prevalence and incidence of $\mathrm{CV}$ events, including acute myocardial infarction (IMA), in a cohort of patients with HIV infection.

Study design and methods: A prospective cohort study started in 2005 in which HIV-infected patients were evaluated for CV risk factors at baseline and annually during regular medical care visits at Second Infectious Diseases Outpatient Clinic. For each patient the following variables were evaluated: age, sex, body mass index, risk factor for HIV acquisition, date of HIV diagnosis, CDC stage, HIVRNA, CD4 T cell count, coinfection with hepatitis B or C virus, cigarette smoking, alcohol abuse, drug addiction, arterial hypertension, diabetes mellitus, dyslipidaemia, previous CV diseases, familiar history of CV diseases, antiretroviral therapy (ARV) and other current therapies. $\mathrm{CV}$ risk was estimated by Framingham risk score (FRS). Chi-square test or Mann-Whitney non-parametric test were used to compare groups respectively for categorical or continuous variables.

Results: until to December 31, 2009, 1,016 patients were enrolled in the study. At enrolment the median age of patients was 41 years (IQR $36-46) ; 74.4 \%$ of patients were males; $57.1 \%$ of patients acquired HIV infection by heterosexual contact, $22.7 \%$ were men having sex with men, and $18.5 \%$ were intravenous drug abusers (DAs). Median duration of HIV disease was 5 years (IQR 1-11). The median CD4 T cell count was $424 / \mathrm{mmc}$ (IQR 242-646) and $45.6 \%$ of patients had an undetectable plasma viremia. $52.7 \%$ of patients were cigarette smokers, and $6.4 \%$ were active DAs $(58.5 \%$ of them were cocaine users). $6.9 \%$ of patients had arterial hypertension, $3.4 \%$ had diabetes mellitus, $26.5 \%$ had hypertriglyceridaemia, $16 \%$ had hypercolesterolaemia. The FRS was $<10 \%$ in $76 \%$ of patients and $>20 \%$ in $6.7 \%$. $66 \%$ of patients were on ARV therapy. Forty-one patients (4\%) had a previous $\mathrm{CV}$ diseases and $12(1.2 \%)$ patients had had a previous $\mathrm{CV}$ event (10 IMA and 2 ictus cerebri). All 12 patients (males 10) had a history of cigarette smoking, were older than patients without previous CV events (44 vs. 41 years, respectively; $p=0.023$ ), and had a longer duration of HIV disease ( 9 vs. 5 years, respectively; $\mathrm{p}=0.017)$. The median follow-up period was 4 years. Over 2,630 person-years, 7 patients experienced a CV event (6 IMA and 1 ictus cerebri) with an incidence rate of $2.6 / 1,000$ person-year (95\% CI 1.16-5.27). All incident patients were males, with a median age of 52 years (IQR 39-59). Only sex and age were found to be significantly associated with $\mathrm{CV}$ events.

Conclusions: CV events in HIV-infected patients represent an increasing concern in HIV infection management. Sex, ageing and duration of HIV disease seem to be important risk factors for the development of $\mathrm{CV}$ events. Further studies are needed to clarify the role of HIV disease in the pathogenesis of the $\mathrm{CV}$ involvement.

\section{PO 59}

CAN ADAPTED PHYSICAL ACTIVITY HELP TO CONTROL AND PREVENT THE LIPODYSTROPHIC SYNDROME IN HIV INFECTION? WALKING TO HEALTH PROTOCOL AT AMEDEO DI SAVOIA HOSPITAL IN TURIN

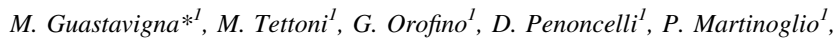
D. Demarie ${ }^{I}$, P. Desiderato ${ }^{1}$, R. Di Frenna ${ }^{1}$, M. Zignin ${ }^{2}$, C. Zignin $^{2}$

${ }^{1}$ Ambulatorio Lipodistrofie e Dismetabolismi HIV correlati, ASLTO2, Turino, Italy; ${ }^{2}$ SUISM, Turino, Italy 
Background: The Lipodystrophic Syndrome (LS) is the expression of HIV infection chronicity and can negatively affect survival, adherence to antiretroviral therapy and quality of life. Currently, about $30 \%$ of HIV positive patients suffer from LS. For these reasons we developed a multidisciplinary diagnostic and therapeutic program, funded by Regione Piemonte, aimed at working on changeable lifestyles, to reverse and control some metabolic alterations and to cope with the distress related to change in body image. The follow-up is based on the patient's needs and is carried out by the multidisciplinary group composed of specialists in HIV, a nutritionist, a dietician, a psychologist, a cardiologist, a kinesiologist and plastic surgeons.

Methods: We chose Nordic Walking (NW) because of its effects on muscle and cardiovascular activation that make it appropriate for metabolic syndrome. We practiced NW twice a week for 12 weeks in a large park in Turin. 13 patients joined the group, 11 Male, 2 Female, aged 33-69 years old. At T0 and T1 (12 weeks) metabolic profile and cardiovascular risk were assessed, the patients underwent spirometry and kinesiological tests (flexibility, monopodalic balance, adapted Cooper test). At T1 a satisfaction and self-evaluation questionnaire was given.

Preliminary results: Patients who maintained a high attendance improved their metabolic profile and kinesiological test results. The questionnaires reported high level of satisfaction about activity, setting and the group's heterogeneous composition and high perception of improvement of physical appearance, quality of life and general health.

Discussion: Our goal is to continue to work on changeable lifestyles (smoking, diet, physical activity, etc.) to improve quality of life, realizing again the APE protocol expanding the participants number. The next step will be to develop the APE as LS prevention in patients who start the antiretroviral therapy.

\section{PO 60}

SWITCHING TO DARUNAVIR/RITONAVIR FROM LOPINAVIR/ RITONAVIR OR FOSAMPRENAVIR/RITONAVIR IMPROVES METABOLIC ASSESSMENT IN HIV POSITIVE PATIENTS

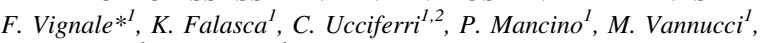
E. Pizzigallo ${ }^{1}, J$. Vecchiet ${ }^{l}$

${ }^{1}$ Infectious Disease Clinic, Department of Medicine and Science of Aging. "G. d'Annunzio" University, Chieti, ${ }^{2}$ Department of Health Sciences, Infectious

Disease Unit, School of Medicine, University of Molise, Campobasso, Italy

Background: Metabolic abnormalities associated with cumulative exposure to cART, have been linked to an increased of cardiovascular risk (CVR) in HIV+ individuals. The aim of this study was to evaluate if the switch to DRV/r from LPV/r or FPV/r in HIV+ patients with HIV-RNA $<400$ copies/ml and metabolic abnormalities improves their lipid and glucidic profile.

Methods: Ten Caucasian HIV+ patients in cART included LPV/r or $\mathrm{FPV} / \mathrm{r}$ for $\geq 12$ months, with high plasmatic levels of total cholesterol (TCh) and triglycerides (TGs) and HIV-RNA $<400$ copies/ml for at least 6 months, were switched to receive DRV/r, without a change in their backbone. Parameters were measured at baseline (T0), after 3 (T3) and 6 months (T6).

Results: After switching, patients showed an improvement of metabolic abnormalities with $18 \%$ reduction of TCh, $15 \%$ for LDL-C, and $52 \%$ decrease in TGs levels whereas HOMA-IR showed a trend to decrease. Cystatin C serum levels statistically decreased. Indexes of hepatic and renal functionality did not change.CD4 cell count increased with statistical significance while HIV-RNA did not change throughout the study.

Conclusions: The switch to DRV/r showed an improvement of metabolic abnormalities and a possible beneficial effect on some factors of CVR. This may also be confirmed by the significant decrease of $11 \%$ in cystatin C, an important predictor of CVR. Furthermore, DRV/r also induced a good immunological response (increase of $21 \%$ of CD4 cell count value), and it was effective in maintaining suppressed viremia. The switch to DRV/r should be considered and preferred in HIV+ cART treated patients showing abnormalities in lipid profile.

\section{PO 61}

THE PERFORMANCE OF INTERFERON-G RELEASE ASSAYS (IGRAS) FOR TB DETECTION IS IMPAIRED IN HIV LATE PRESENTATION

I. Sauzullo ${ }^{2}$, F. Mengoni ${ }^{2}$, R. Rossi $i^{2}$ A. Ermocida ${ }^{2}$, M. Lichtner ${ }^{1}$, G. D'Ettorre ${ }^{2}$, V. Vullo ${ }^{2}$, C.M. Mastroianni ${ }^{I}$

${ }^{1}$ Sapienza Università, Polo Pontino, Latina, Italy; ${ }^{2}$ Sapienza Università di Roma, Unità di Malattie Infettive, Rome, Italy

Background: The introduction of the interferon-gamma release assays (IGRAs) for diagnosing TB infection gave promising results in immunocompetent patients, but the value of these tests in HIV/TB coinfection is still unclear, especially in late presentation. The aim of this study was to evaluate the performance of commercial QuantiFERON-TB Gold in Tube (QFT-G) as a tool to identify TB infection in patients with late presentation of HIV.

Methods: A total of $197 \mathrm{HIV}$-infected individuals with suspected TB were tested with QFT-G. The median CD4 count of the HIV-infected population was 219 cells/ $\mu$ l (range $4-995$ cells $/ \mu 1$ ), and $45(22 \%)$ patients had a CD4 count $<200$ cells/ $\mu$ l. Statistical analysis in the patients was performed using Spearman rank correlation tests.

Results: The QFT-G test was positive in $35(18 \%)$ subjects, negative in $122(62 \%)$, and indeterminate in $40(20 \%)$ subjects. The level of agreement between QFT-G and TST was 68\% $(?=0.30)$ and QFT-G sensitivity was $66 \%$ (95\% CI 47-82). Patients with positive QFT-G results had significantly lower $\mathrm{CD} 4$ counts than those with a negative QFT-G (median 177 vs. 467 cells $/ \mu 1, p=0.001$ ). The lowest CD4 counts (median 97 cells $/ \mu \mathrm{l}$ ) were found in subjects with indeterminate QFT-G results. The univariate analysis confirmed that a CD4 count $<200$ cells $/ \mu$ l was strongly associated with an indeterminate result (OR 5.80, 95\% CI 2.13-16.18, p < 0.001).

Conclusions: These data indicated that the severe CD4 count deficit affects the performance of QFT-G and it is a limitation of this test in subjects with late presentation of HIV infection. Thus, the performance of immunologic assays and its potential as a rule-out test for active TB is impaired in HIV-infected patients with advanced immunosuppression.

PO 62

HCV OCCULT INFECTION IN HIV POSITIVE SUBJECTS: DOES IT EXIST?

F. Di Martino ${ }^{I}$, N. Coppola ${ }^{2}$, S. Martini ${ }^{*}{ }^{1}$, M. Pisaturo $^{2}$, G. Tonziello $^{2}$, A. Masiello ${ }^{3}$, A. Filippini ${ }^{1}$, A. Cascone ${ }^{1}$, E. Sagnelli ${ }^{2}$, P. Filippini ${ }^{1}$

${ }^{1}$ UOC Diagnosi e Terapia AIDS della AOU della Seconda Università degli Studi di Napoli, Naples, Italy; ${ }^{2}$ Clinica di Malattie Infettive della AOU della Seconda Università degli studi di Napoli, Naples, Italy; ${ }^{3}$ AORN G. Moscati, Avellino, Italy

Introduction: HCV occult hepatitis is defined by the presence of HCV-RNA in liver issue and/or peripheral blood mononucleated cells (PBMC) without seric anti-HCV and HCV-RNA. It has been found in subjects with sustained virological response after anti-viral therapy and in subjects with high levels of seric transaminases of unknown origin.HCV occult infection has also been found in immunocompromised hemodialysed patients, without seric anti-HCV and HCVRNA with persistent high levels of transaminases.

Aims: To evaluate the prevalence of $\mathrm{HCV}$ occult infection among HIV positive (+)/anti-HCV/HCV-RNA negative patients, by searching HCV-RNA in PBMC.

Patients and methods: We have enrolled 45 consecutive HIV+/antiHCV/HCV-RNA negative patients (Case Group),observed from 
January to April 2009. Risk factors for HIV were sexual contacts (91.2\%),intra-venous drug abuse (6.6\%),vertical transmission (2.2\%).From each patient we have drawn a blood sample and samples of PBMC, frozen at $-80^{\circ} \mathrm{C}$.For each patient we have detected biochemical markers of liver function, $\mathrm{HBV}$ and $\mathrm{HCV}$ markers, CD4 cells count, HIV-RNA at the enrollment, CD4 nadir and therapeutic history. As Control Goup, we have enrolled seven consecutive HIV neg/anti-HCV/HCV-RNA pos patients, observed at the time of liver biopsy. We have detected HCV-RNA on plasma and PBMC of both groups of patients. In all PBMC samples, we have also searched mRNA of G6PDH, present in every human cell.

Results: Among Cases, every patient showed the alteration of at least a marker of liver function. At the enrollment, the median value of CD4 was 717 cells $/ \mu \mathrm{L} ; 84.4 \%$ of the subjects were on HAART. In all our Cases, HCV-RNA was not detectable in PBMC, while it was present in all controls. Both in cases and in controls, the detection of mRNA of G6PDH was positive in all patients.

Conclusions: The anti-HIV+/anti-HCV neg patients enrolled could have been exposed to HCV by parenteral and sexual contacts. Though there is a high risk, $\mathrm{HCV}$ occult infection has not been observed in our 45 cases. It is possible to exclude a low sensitivity of our method, infact HCV RNA is positive in PBMC samples of all controls and mRNA of G6PDH is positive in all PBMC samples both in Cases and Controls. The absence of HCV occult infection observed in our cases may be related to the good immune condition of patients enrolled, critical for the control of HCV replication in plasma and PBMC.It has not been possible to examine liver issue in these patients without clear indication to liver biopsy.

\section{PO 63}

EPSTEIN-BARR VIRUS LOAD AND IMMUNE ACTIVATION IN HIV1 INFECTED PATIENTS

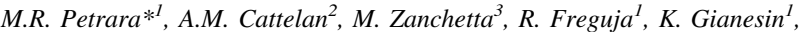

F. Carmona ${ }^{3}$, A. De Rossi ${ }^{l}$

${ }^{I}$ Dipartimento di Scienze Oncologiche e Chirurgiche, AIDS Reference Centre, Università degli Studi di Padova, Padua, Italy; ${ }^{2}$ Divisione di Malattie Infettive, Ospedale di Rovigo, Rovigo; ${ }^{3}$ Istituto Oncologico Veneto, Padua, Italy

Purpose: Patients infected with HIV-1 are at high risk of developing virus-associated malignancies. The introduction of antiretroviral therapy (ART) has greatly modified the course of HIV-1 infection. However, the impact of ART seems to be less favourable on lymphoproliferative disorders associated with EBV than on other AIDSassociated illness, including Kaposi sarcoma. The aim of this study was to estimate the relationship between EBV levels and other viralimmunological parameters in HIV-1 infected subjects.

Patients and methods: $156 \mathrm{HIV}-1$ infected patients who consecutively attended the Division of Infectious Disease of Rovigo Hospital from July 2007 to December 2009 were included in this study. HIV-1 RNA was quantified by COBAS Taqman HIV-1 assay. HIV-1 DNA in peripheral blood mononuclear cells (PBMC) was determined by real-time PCR. EBV type 1 and EBV type 2 were quantified on PBMC samples by Multiplex Real-Time PCR. Lipopolysaccharide (LPS), a marker of microbial translocation, was determined in plasma samples using a chromogenic assay (Limolus Amebocyte Lysate). B-cell activation was analyzed by flow cytometry using monoclonal antibodies CD19PerCP, CD86APC, CD69PE.

Results: EBV was detected in 114 patients, and in all but 3 cases was EBV type 1. The median [IQR] EBV-DNA load was 43.5 (1-151) copies/105 PBMC. $44 \%$ of patients had CD4 cell counts $>500$ cells $/ \mu 1$ and $28 \%$ had undetectable HIV-1 plasma viremia $(<50$ copies $/ \mathrm{ml})$. The EBV-DNA level was significantly higher in patients with CD4 $<500$ cells/ $\mu$ l than in those with CD4 $>500$ cells/ $\mu$ [ 72 (15-320) vs. 18 (1-68) copies/105 PBMC; p $<0.0001]$, and in patients with detectable HIV-1 RNA than in those with undetectable plasma viremia [56 (9-300) vs. 18 (1-52) copies/105 PBMC; $p=0.001]$.
Interestingly, levels of EBV-DNA were higher in the group of patients with CD4 $>500$ cells $/ \mu$ l and high HIV-1 plasma viremia $(>1,000$ copies $/ \mathrm{ml})$ than in those with low HIV-1 load, regardless of the immunological status $[48(5-153)$ vs. 18 (1-60) copies/105 PBMC; $p=0.015]$. Furthermore, patients with high EBV loads (up to 75th percentile) had higher levels of HIV-1 DNA [40 (1-132) vs. 10 (1-76) copies/105 PBMC; $\mathrm{p}=0.050]$ and higher levels of LPS [130 (88-244) vs. $98(81-134) \mathrm{pg} / \mathrm{ml} ; \mathrm{p}=0.024]$ than patients with low EBV loads. B cell activation in patients with high EBV load was confirmed by immunophenotyping; three of these patients developed B-cell lymphoma.

Conclusions: These findings suggest that HIV-1 viremia and immune activation play an important role in the B-cell stimulation and expansion of EBV-infected cells. Persistant HIV-1 viremia, despite immunoreconstitution, may represent a risk factor for the onset of EBV-related malignancies.

\section{PO 64}

EFFECT OF HCV THERAPY ON IMMUNOLOGICAL HOMEOSTASIS IN HIV/HCV CO-INFECTED PATIENTS

M. Airoldi ${ }^{1}$, P. Nasta ${ }^{2}$, F. Maggiolo ${ }^{3}$, S. Di Gianbenedetto ${ }^{4}$, N. Ladisa ${ }^{5}$,

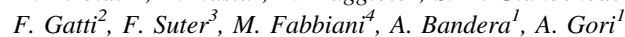

${ }^{1}$ Division of Infectious Diseases, San Gerardo Hospital, University of MilanBicocca, Monza, Italy; ${ }^{2}$ Institute of Infectious and Tropical Diseases, University of Brescia, Brescia, Italy; ${ }^{3}$ Ospedali Riuniti di Bergamo, USC Malattie Infettive, Bergamo, Italy; ${ }^{4}$ Institute of Clinical Infectious Diseases, Catholic University, Rome, Italy; ${ }^{5}$ Institute of Infectious Diseases, University of Bari, Bari, Italy

Background: The effect of HCV on HIV infection progression remains unclear and its impact on immune reconstitution after antiretroviral therapy (cART) is partially unknown. We evaluated the effect of the response to HCV treatment on CD4 and CD8 cell counts in a cohort of HIV/HCV co-infected adults.

Methods: We retrospectively analyzed an Italian multi-site cohort of HIV-infected patients with HCV infection (HCV-RNA positive). To be eligible patients had to be on stable cART and treated with peginterferon and ribavirin for at least 3 months. CD4 and CD8 variation over time were analysed using a general linear model comparing three different groups of patients: sustained virological responders (SVR), non responders (NR) and relapsers defined according to response to HCV treatment. CD4 counts at the end and after 12 months from the end of HCV therapy were considered.

Results: 305 subjects responded to the selection criteria were included. Median age was 41 years, $79 \%$ were males. 163 patients $(53 \%)$ accomplished the definition of SVR, 88 (29\%) were NR and 54 (18\%) relapsers. At baseline mean CD4 cell counts were 532 (SD 242) cells/ $\mu \mathrm{l}$ in the SVR group, 575 (SD 264) cells/ $\mu 1$ in the NR group and 554 (SD 269) cells $/ \mu \mathrm{l}$ in relapsers $(\mathrm{p}=\mathrm{ns})$. At the end of treatment, absolute CD4 count was 352 (SD 188) cells/ $\mu$ l in SVR, 385 (SD 171) cells $/ \mu 1$ in NR and 332 (SD 166) cells/ $\mu 1$ in relapser $(p=n s)$. After 12 months from the end of HCV treatment CD4+ increased to 565 (SD 245) cells $/ \mu 1,614$ (SD 301) cells $/ \mu 1,501$ (SD 215) cells $/ \mu 1$ in SVR, NR and relapsers, respectively $(p=n s)$. Difference in CD4 counts between the end of HCV treatment and 12 months later were 190 (SD 203) cells/ $\mu 1$ in SVR group, 230 (SD 224) cells/ $\mu 1$ in NR and 188 (SD 138) cells/ $\mu \mathrm{l}$ in relapsers $(\mathrm{p}=\mathrm{ns})$. No statistically significant difference was observed considering the percentage of CD4+, CD8 cell absolute count, $\mathrm{CD} 8$ percentages and $\mathrm{CD} 4 / \mathrm{CD} 8$ ratio in the three groups at each time point.

Conclusions: HCV treatment markedly reduced CD4 cell counts in all patients. After 12 months from the end of hepatitis treatment, CD4 cell counts returned to baseline values irrespective of $\mathrm{HCV}$ treatment response. It can be hypothesized that response to HIV treatment and not to HCV therapy is the driving force for immune restoration in coinfected patients. 


\section{PO 65}

VISCERAL LEISHMANIASIS: DEMOGRAPHIC, CLINIC AND THERAPEUTIC PREDICTORS OF INFECTION RECURRENCES

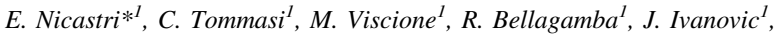
M.G. Paglia ${ }^{I}$, P. Ghirga ${ }^{1}$, A. Corpolongo ${ }^{I}$, A.L. Gallo ${ }^{I}$, P. Narciso $^{I}$ ${ }^{I}$ National Institute for Infectious Diseases, IRCCS, "Lazzaro Spallanzani", Rome

Background: In the Mediterranean area, visceral leishmaniasis is prevalently caused by Leishmania infantum and it is associated to HIV infection in a $25-70 \%$ of cases. Visceral leishmaniasis is susceptible of antiparasitic treatment but usually HIV-infected patients present atypical clinical presentation, lower rate of response to antiparasitic drugs and higher risk of relapse than non-HIV subjects.

Objective: To evaluate the epidemiologic, clinic and therapeutic factors associated to visceral leishmaniasis recurrences in patients with or without HIV infection.

Methods: A retrospective analysis of visceral leishmaniasis cases treated at National Institute for Infectious Diseases "Lazzaro Spallanzani" from January 2006 to December 2009 was performed. Patients were divided in two groups according to HIV status. Demographic, clinical e therapeutic characteristics were recorded and analyzed using uni- and multivariate analyses as appropriate.

Results: We analyzed 33 patients with visceral leishmaniasis. Of them, $26(29 \%)$ were male, with a mean age of $44( \pm 12)$ years. One of 33 patients presented visceral leishmaniasis with cutaneous involvement. Twenty (61\%) patients were HIV-infected (group A), 13 (39\%) were HIV negative (group B). No differences in sex, age and race between groups were found. Patients in group A were more frequently co-infected with hepatitis B or $\mathrm{C}$ than patients in group $\mathrm{B}$ (40 vs. $7 \%$, respectively; $p=0.008$ ). All patients received standard treatment with liposomial amphotericine B. Relapses occurred in the $27 \%$ of patients. The incidence of relapse was significantly higher in group A than B (78 vs. $22 \%$, respectively; $p=0.009$ ). Other factors associated to relapse risk were: co-infection with hepatitis $\mathrm{B}$ or $\mathrm{C}$ $(\mathrm{p}=0.014)$ and age at the first diagnosis $(\mathrm{p}=0.035)$. The statistical significance was confirmed at uni- and multivariate analysis. The prophylactic therapy with miltefosine or liposomial amphotericine was administered in 3 of 20 patients in group A; no patients in group $B$ received prophylaxis. No statistical correlation between use of chemoprophylaxis and relapse was found.

Discussion: Patients with HIV infection had a higher risk of relapse than without HIV. The HBV or HCV co-infection and the older age at the first diagnosis resulted independently associated to risk of relapse. Several analyses are necessary to better evaluate the role of chemoprophylaxis in order to reduce the relapse in HIV patients.

\section{PO 66 \\ TUBERCULAR GLUTEUS ABSCESSES: A RETURN TO THE EARLY 20TH CENTURY, OR A CONSEQUENCE OF NEW, UNPRECEDENTED BEHAVIORS? \\ E.M. Gabrielli ${ }^{*}$, F. Zanini ${ }^{l}$, S. Merli ${ }^{2}$, A. Riva ${ }^{I}$, M. Galli ${ }^{l}$, C. Gervasoni ${ }^{I}$ \\ ${ }^{1}$ Clinical Science Department, Section of Infectious Diseases, University of Milan, L.Sacco Hospital, Milano; ${ }^{2} I I$ Division of Infectious Diseases, L.Sacco Hospital, Milano}

Background: Abscess formation is one of the possible manifestations of extra-pulmonary tuberculosis (TB). Tubercular abscesses may appear anywhere in the body, but are most commonly found in the spines, hips, lymph nodes or in the genital area. On the contrary, gluteal localization, without bone involvement, is an atypical and rare presentation in the general population, that was never reported in people living with HIV/AIDS.

Patients and methods: We molecularly characterized the Mycobacterium tubercolosis strains isolated from gluteus abscesses of five transgender HIV-positive patients who received years or months earlier multiple silicone fluid injections in the buttocks for cosmetic purpose, and compared the strains with previous pulmonary isolates using Spolygotyping and MIRU-VNTRR 12 loci.

Results: In the last year, diffuse swelling of the buttocks with overlying skin lesions associated with seropurulent discharge was observed in five HIV-infected patients, all on antiretroviral (ARV) treatment with CD4 count higher than 300 cells/ $\mu$ l and undetectable HIV-RNA. Unexpectedly, M. tuberculosis was isolated from all the patients. All the patients had been treated for pulmonary TB years earlier. At time of abscess presentation chest radiography and sputum culture for $M$. tuberculosis were negative in all but one patients and no bone involvement was detected. The patients received rifampin, isoniazid, pyrazinamide and ethambutol for 2 months, followed by rifampin and isoniazid for further 4 months. Since previous pulmonary strains were available, genotypical identity between the abscess strain and the previous pulmonary isolate was demonstrated with molecular biology techniques.

Conclusions: The cases described are extremely unusual because the patients suffered from pulmonary TB more than 5 years before presenting abscess formation. In our opinion, muscle TB develops as the result of haematogenous or lymphatic spread of M.tuberculosis from a still active pulmonary focus in one case or from healed foci in the other four. It is possible that the decreased immunity associated to HIV infection might have possibly flared up an underlying TB infection, even if we cannot exclude that silicon oil promotes the growth of M.tuberculosis. In conclusion we should consider the possibility of TB in any transgender patient who underwent silicone fluid injections in the buttocks presenting gluteus abscesses.

\section{PO 67}

SYPHILITIC HEPATITIS IN A COHORT OF HIV-INFECTED SUBJECTS: INCIDENCE AND RISK FACTORS

R. Rossotti ${ }^{\prime}$, C. Molteni ${ }^{I}$, P. Bonfanti ${ }^{I}$, A. Orani ${ }^{I}$

${ }^{1}$ SC Malattie Infettive e Tropicali, AO "A. Manzoni", Lecco

Introduction: Described for the first time by Paracelsus in 1585 , syphilitic hepatitis has been classically depicted as disproportionately elevated alkaline phosphatase (ALP) level in the setting of a secondary syphilis with rash. In the last 30 years it has been poorly studied, especially in HIV-infected subjects: to date, only ten series of acute hepatitis among HIV patients have been reported and risk factors associated with this condition have not been fully investigated. Nevertheless, given the recent syphilis upsurge, in particular among men who have sex with men, it deserves new interest. Aim of our study is to analyze incidence of syphilitic hepatitis in an HIV-infected cohort evaluating possible risk factors associated with elevated hepatic values.

Methods: All patients attending our Outpatient HIV Clinic from January, 012009 to December, 312010 were tested for T. pallidum antibodies; those who tested positive, were further analyzed with RPR and TPPA. Subjects with positive RPR but with negative serology for $\mathrm{HBV}$ and $\mathrm{HCV}$ were included in this analysis. Incidence and risk factors were assessed with descriptive and regression statistical approaches.

Results: During the 24 months study period, 605 patients were tested and 35 resulted RPR-positive (incidence 5.8\%); 9 subjects were excluded because of a HBV/HCV co-infection. Among the 26 included (Table 1), 4 had abnormal ALT values ( $>2$ ULN): hepatitis incidence was $11.4 \%$ among all syphilis cases, $0.7 \%$ in the whole cohort. Figure 1 shows cases of elevated ALT values in our Outpatient Clinic: syphilis represents a minor cause of hypertransaminasemia (2.9\%). Syphilis is even a minor cause of elevated ALP levels (1.9\%). Syphilitic hepatitis heals within 3-6 months after a proper antibiotic 
treatment (Fig. 2). Risk factors associated with elevated ALT and ALP values are shown in Table 2.

Discussion: Syphilitic hepatitis is an uncommon condition; in our Clinic, ALP values seldom increased to clinically significant values. Given the small number of cases, the statistical analyses lack of adequate power; symptomatic (secondary or tertiary) syphilis infection is the only risk factor associated with raised ALT values. Although uncommon, syphilis should be taken into account when evaluating unexpected high transaminases levels; further studies with larger samples are needed to recognize more effectively risk factors associated with this hepatic involvement.

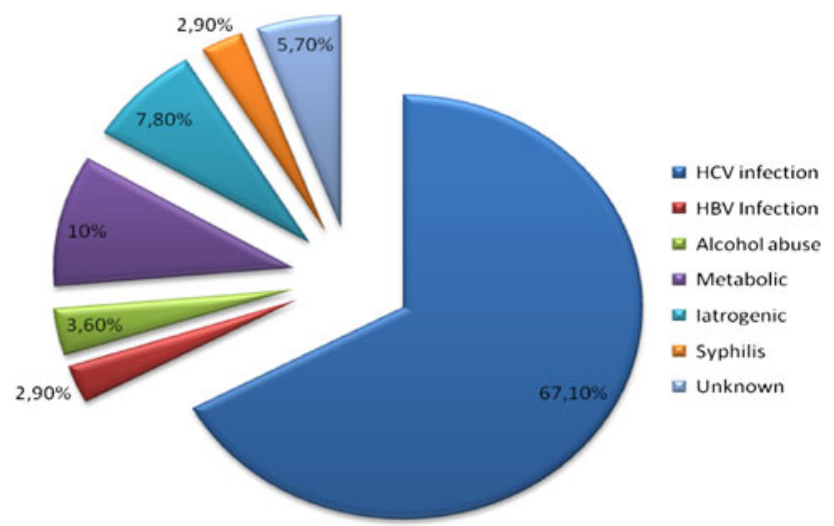

Fig. 1 Reasons of elevated ALT values ( $>2$ ULN); total patient included $\mathrm{N}=140$

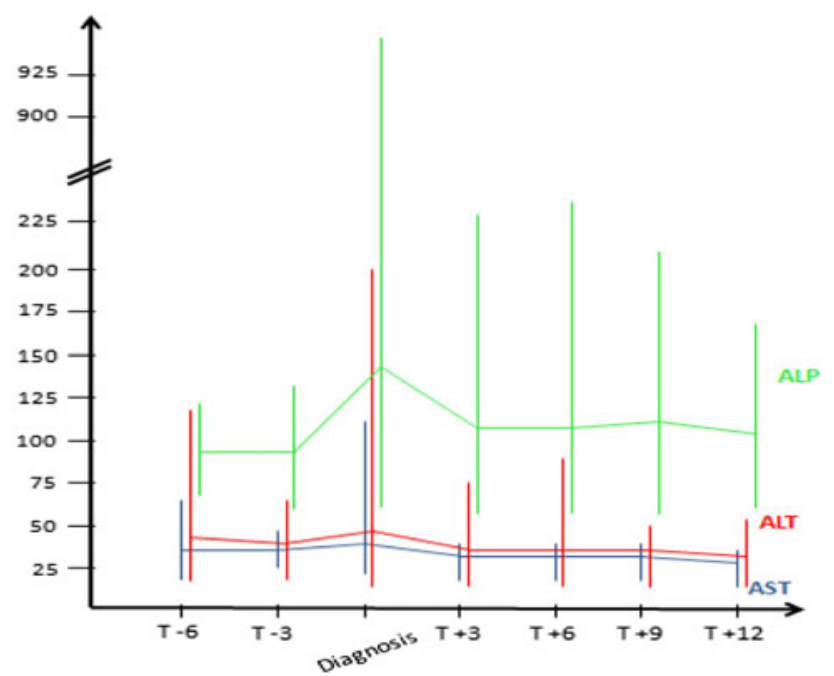

Fig. 2 AST, ALT and ALP average values across time (with minimum and maximum values) starting 6 months before the syphilis serologic diagnosis and with a follow up of 12 months
Table 1 Study population features $(\mathrm{N}=26)$. NNRTI included nevirapine and efavirenz; PI included lopinavir/ritonavir, fosamprenavir/ ritonavir and atazanavir/ritonavir. HIV-RNA was assessed as percentage of undetectability $\left(<1.3 \log _{10}\right)$ and average viral load value (naïve subjects and experienced patients with virological failure)

Age (years)

Average

Median \pm SD

$43 \pm 8.97$

Male gender $(\%)$

100.0

Symptomatic syphilis infection (\%)

34.6

Syphilis infection-relapse $(\%)$

50.0

Naïve (\%)

23.2

Experienced

NNRTI (\%)

38.4

PI $(\%)$

34.6

RAL (\%)

3.8

CD4 (average)

490

HIV-RNA $\left(\log _{10}\right)$

$<1.3(\%)$

Average (>1.3)

3.5

RPR ( $\log _{2} ;$ mode)

6

TPPA (mode)

AST (average at diagnosis)

ALT (average at diagnosis)

Transaminases $>2$ ULN $(\%)$

ALP (average at diagnosis)

144.1

ALP > ULN (\%)

15.4

Total bilirubin (average at diagnosis)

0.86

Aspartate aminotransferase (AST, normal range 0-40 U/L); alanine aminotransferase (ALT, normal range 0-40 U/L); alkaline phosphatase (ALP, normal range 40-128 U/L); total bilirubin (normal range $0-1.1 \mathrm{U} / \mathrm{L})$

Table 2 Risk factors associated with abnormal ALT and ALP values

\begin{tabular}{|c|c|c|c|c|c|c|}
\hline & \multicolumn{3}{|l|}{ ALT } & \multicolumn{3}{|l|}{ ALP } \\
\hline & OR & $95 \% \mathrm{CI}$ & $\mathrm{P}$ & OR & $95 \% \mathrm{CI}$ & $\mathrm{P}$ \\
\hline $\begin{array}{l}\text { Symptomatic syphilis } \\
\text { infection }\end{array}$ & 16.00 & $1.22-210.60$ & 0.014 & 1.17 & $0.09-14.52$ & $>0.02$ \\
\hline $\begin{array}{l}\text { Syphilis infection- } \\
\text { relapse }\end{array}$ & 0.24 & $0.02-2.78$ & $>0.025$ & 1.00 & $0.11-8.73$ & $>0.025$ \\
\hline HAART & 3.75 & $0.40-35.54$ & $>0.025$ & 0.29 & $0.03-2.72$ & $>0.025$ \\
\hline \multicolumn{7}{|l|}{ CD4 } \\
\hline $0-350$ & 1.78 & $0.14-23.40$ & $>0.025$ & 8.00 & $0.69-92.71$ & $>0.025$ \\
\hline $350-500$ & 2.17 & $0.24-19.28$ & $>0.025$ & 2.60 & $0.28-23.82$ & $>0.025$ \\
\hline$>500$ & 0.30 & $0.03-3.43$ & $>0.025$ & Ns & & \\
\hline \multicolumn{7}{|l|}{ HIV-RNA $\left(\log _{10}\right)$} \\
\hline$<1.3$ & 0.73 & $0.08-6.31$ & $>0.025$ & Ns & & \\
\hline $1.5-5.0$ & 0.57 & $0.05-6.61$ & $>0.025$ & 10.50 & $0.84-130.67$ & $>0.025$ \\
\hline$>5.0$ & 6.0 & $0.29-124.11$ & $>0.025$ & 5.67 & $0.27-117.45$ & $>0.025$ \\
\hline \multicolumn{7}{|l|}{ RPR $\left(\log _{2}\right)$} \\
\hline$>5$ & 3.33 & $0.29-38.08$ & $>0.025$ & 0.80 & $0.09-7.00$ & $>0.025$ \\
\hline \multicolumn{7}{|l|}{ TPPA } \\
\hline$>5,120$ & 0.80 & $0.06-9.92$ & $>0.025$ & 0.86 & $0.07-10.67$ & $>0.02$ \\
\hline
\end{tabular}




\section{PO 69}

EFFECTS OF CADMIUM AND GC-MACROPHAGE ACTIVATING FACTOR (GCMAF) ON INTRACELLULAR HIV TARGETS IN NORMAL AND TRANSFORMED HUMAN BREAST CELLS G. Morucci $^{l}$, T. Punzi ${ }^{\text {* }}{ }^{1}$

${ }^{1}$ Department of Anatomy, Histology and Forensic Medicine, University of Firenze, Florence, Italy

Breast cancer is of particular importance among non-AIDS defining cancers (NADCs), because of its incidence in industrialized and developing countries. Here we studied the effects of a known human carcinogen, the highly persistent heavy metal cadmium, on cell proliferation, angiogenesis, and on two of the main intracellular targets of HIV, heat shock protein 90 (hsp90, targeted by Tat protein, known to be imported into the nucleus of human breast cancer cells), and poly(ADP-ribose) polymerase (PARP, involved in DNA repair and oxidative stress associated with HIV infection). The effects of nontoxic doses of cadmium $(1-10 \mu \mathrm{M})$ on intracellular HIV targets were studied in human breast cancer cells (MCF-7) and in their normal counterpart (MCF-10A). The effects of Gc-Macrophage Activating Factor (GcMAF), a protein demonstrated to be effective in HIV and breast cancer treatment (J Med Virol 81:16-26, 2009. Int J Cancer 122: 461-7, 2008) were also studied in MCF-7 cells. Cell proliferation was studied by 5-bromo-deoxiuridine labelling and by MTTbased assay. Hsp90 $\beta$ expression was evaluated by immunohistochemistry. PARP expression was studied by immunohistochemistry and Western blot. Angiogenesis was studied in chick embryo chorionallantoic membrane (CAM). Proliferation of normal and transformed cells in serum-starved medium was inhibited by cadmium in dose-dependent manner. GcMAF (1 ng/ml) significantly inhibited MCF-7proliferation. The effect of cadmium was partially reversed by zinc. Hsp90 $\beta$ and PARP expression levels were increased by cadmium in dose-dependent manner in both types of cells, thus mimicking the effects of HIV on those intracellular targets. The effects of cadmium on angiogenesis were opposite in the two cell lines; MCF-7-induced angiogenesis in CAM was inhibited, whereas MCF-10-induced angiogenesis was stimulated. GcMAF $(1 \mathrm{ng} / \mathrm{ml})$ significantly inhibited MCF-7-induced angiogenesis. These results indicate that cadmium and HIV recognize as intracellular molecular targets two of the principal regulators of cell responses to stress, i.e. hsp90 and PARP. In human breast cancer cells, increased expression of hsp90 and PARP was associated with reduced cell proliferation and inhibition of angiogenesis. Since cadmium and GcMAF exerted similar effects on MCF-7 and on MCF-7-induced angiogenesis, we hypothesize that hsp90 and PARP are involved in the GcMAF signalling pathway. These results also open the perspective of studying $\mathrm{HIV}$-associated angiogenesis in NADCs with the goal of controlling the progression of NADCs via inhibition of angiogenesis.

\section{PO 70}

\section{ANAL MALIGNANCIES ASSOCIATED TO CONDYLOMA} ACUMINATA: DIFFERENCES BETWEEN HIV+ AND HIVPATIENTS

M. Mistrangelo* ${ }^{1}$, I. Dal Conte ${ }^{2}$, P. Cassoni ${ }^{3}$, R. Senetta ${ }^{3}$, S. Delmonte ${ }^{4}$,

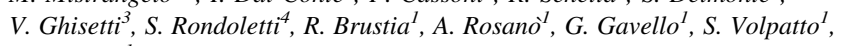
M. Morino ${ }^{l}$

${ }^{I}$ Digestive and Coloproctological Surgical Department and Centre of Minimally Invasive Surgery, University of Turin, Molinette Hospital, Turin, Italy; ${ }^{2}$ Infectious Diseases Department, University of Turin, Amedeo di Savoia Hospital, Turin, Italy; ${ }^{3}$ Department of Biomedical Sciences and Human Oncology, University of Turin, Molinette Hospital, Turin, Italy;

${ }^{4}$ Dermatological Department, University of Turin, Turino, Italy

Introduction: Human Papillomavirus (HPV) infection is emerging as an important factor in the oncogenesis of various squamous cancers.
The incidence of HPV-associated anal cancer has recently increased, largely attributed to immunocompromised states such as human immunodeficiency virus (HIV) infection. HPV infections may cause squamous intraepithelial neoplasia, which may progress from low grade to high grade and may be found in areas adjacent to squamous cell carcinoma. HPV DNA was found in $88 \%$ of anal cancer in one study and in more than $90 \%$ of cervical squamous cell carcinoma. We present our experience in the treatment of anal condylomata, and in particular our histological findings after surgery.

Patients and methods: At our Department 1,153 patients (758 M and $395 \mathrm{~F}$ ) were diagnosed as affected by anal and perianal condylomata in a period from October 1999 and October 2010. Mean age was 34.7 years. 165 patients $(14.3 \%)$ were $\mathrm{HIV}+, 6.1 \% \mathrm{HBV}+$ and $2.6 \%$ $\mathrm{HCV}+.94 \%$ presented a perianal localization, $62 \%$ an endoanal (4.6\% only endoanal), and $35.8 \%$ a genital localization. 967 patients were submitted to surgery in one or more sessions.

Results: Histological exam revealed a degeneration in 62 patients (6.4\%). 48 of these patients were HIV+ (77.48\% of degenerations); and 2 patients were immunosuppressed (3.2\%). Other 24 patients were immunocompetent. When we consider all series degenerations occurred in $29 \%$ of HIV+ patients versus $2.4 \%$ of HIV-patients. In particular histological exam revealed 13 AIN I; 7 AIN II; 6 AIN III; 11 Buschke Lowenstein neoplasms, 15 Bowen disease or Bowenoid papillomatosis; 3 carcinomas in situ; 7 squamous or basaloid carcinomas ( 3 of these were multifocal). Patients with diagnosis of Buschke Lowenstein tumour was submitted to radical surgery; patients with anal carcinoma to RT + CT or to surgical excision; and other patients to surgical excision. All patients are actually in follow up.

Conclusions: HPV related perianal condylomata become increasingly frequent. Clinical diagnosis is simple. However, histopathological examination should be performed to confirm the diagnosis and to detect precancerous or cancerous changes, mainly in HIV+ patients. Molecular biological techniques allow identification of the HPV genotype so detecting the oncogenic strains. An accurate follow up is mandatory for patients revealed as malignancies to prevent transformation in anal carcinoma. High resolution anoscopy is mandatory in these patients, and in all HIV+ patients, mainly if MSM.

\section{PO 71}

BREAST CANCERS AND LUNG CANCERS IN HIV-POSITIVE PATIENTS FROM 1999 TO 2009 IN THE INSTITUTE OF INFECTIOUS DISEASES OF BRESCIA: LESSONS LEARNED FROM CLINICAL CASES

A. Vavassori $^{*}$, A. Ferraresi ${ }^{I}$, A. Calabresi ${ }^{I}$, I. Izzo ${ }^{I}$, F. Castelnuovo ${ }^{I}$, I. El

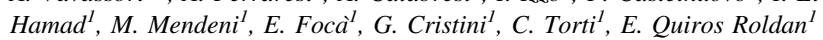
${ }^{I}$ Department of Infectious Diseases, University and Spedali Civili of Brescia, Brescia, Italy

Background: Breast and lung cancers were the most frequent nonAIDS-non-virus related tumors in our integrated database (also presented in this Conference) in the period 1999-2009.

Methods: Descriptive case series analysis.

Results: Ten breast cancers and 23 lung cancers were diagnosed among 4,937 HIV-infected patients. Breast cancers: At presentation, six patients were between 25 and 44 years-old, three between 45 and 64 and one male was 66. Cancer diagnoses were made at HIV diagnosis in only two and five patients were already on cART. CD4+ T-cell counts at cancer ranged between 70 and $1,514 / \mathrm{mm}^{3}$ with nadir between 12 and 409/ $\mathrm{mm}^{3}$. All were adenocarcinoma (8/10 ductal). Over a follow-up of 1-6 years, all patients but one survived. Lung cancers: At presentation, five patients were between 30 and 44 yearsold, ten between 45 and 59 and eight between 60 and 74 . Fifteen patients were smokers, while two patients were not (both on 
virologically successful HAART). Cancers occurred concomitantly with HIV diagnosis in $5 / 23$ patients, while the remaining patients were already on follow-up ( $\mathrm{n}=18$ on cART). Notwithstanding this, advanced stages (IIIB or IV) were found in all patients but 2. CD4+ T-cell counts ranged between 21 and $784 / \mathrm{mm}^{3}$ with a nadir between 19 and $583 / \mathrm{mm}^{3}$. The most frequent type was squamous-cell carcinoma. Only two patients survived reaching 25 and 61 months from diagnosis.

Conclusions: Breast cancers: Our data suggest that screening policy for breast cancer should be started earlier in HIV patients than currently recommended in the general population ( $>40-50$ years). Lung cancers: Letality of lung cancer appeared to be very high probably because diagnosis was made at advanced stages. Moreover, almost all patients were already followed at cancer diagnosis. Therefore, innovative screening policies for lung cancer may warrant to be experimented and, possibly, implemented in our patients.

PO 72

\section{NON-HODGKIN LYMPHOMA IN HIV PATIENTS: INFECTIOUS} COMPLICATIONS AND SURVIVAL RATE

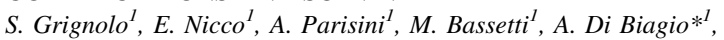

F. Ballerini ${ }^{I}$, C. Viscoli $^{I}$

${ }^{I}$ Department of Infectious Diseases, University of Genoa, San Martino

Hospital, Genoa, Italy

Background: Non-Hodgkin lymphoma (NHL) has become the most common HIV-associated malignancy. Aim of the study was to evaluate the survival rate and the incidence of infectious complications in patients with HIV and NHL and compared with a cohort of patients without HIV.

Methods: Retrospective case-control study (match 1:2) of high grade NHL in HIV-population and HIV-negative patients followed in our Hospital, from 1996 to 2008 has been performed. Age, stage, histotype, B-symptoms, chemotherapy-related toxicity, survival at week 6 , 12,24 and 48 and infectious complications were examined using univariate analysis.

Results: A total of 15 cases and 30 controls were successively selected. The main differences between groups observed are summarized in Table 1 .

Table 1

\begin{tabular}{|c|c|c|c|}
\hline & $\begin{array}{l}\text { NHL-HIV } \\
+\end{array}$ & $\begin{array}{l}\text { NHL-HIV } \\
-\end{array}$ & $\begin{array}{l}\mathrm{p} \\
\text { value }\end{array}$ \\
\hline Median age (years) & 40.7 & 53.8 & 0.003 \\
\hline Survival at week $12(\%)$ & 85.7 & 100 & 0.03 \\
\hline Survival at week $24(\%)$ & 64.3 & 96.7 & 0.004 \\
\hline Survival at week $48(\%)$ & 38.5 & 90 & 0.0004 \\
\hline $\begin{array}{l}\text { Low Respiratory tract infections } \\
\text { (LRTI) }\end{array}$ & $8 / 57.1 \%$ & $5 / 16.7 \%$ & 0.002 \\
\hline $\begin{array}{l}\text { Infection positive outcome } \\
\quad(30 \text { days })\end{array}$ & $14 / 63.6$ & $21 / 95.5$ & 0.03 \\
\hline
\end{tabular}

No differences in histotypes (the most common was diffuse large B-cell lymphoma), stage, B-symptoms, chemotherapy-related toxicity and infectious complications rate has been observed.

Conclusions: HIV-infected patients with NHL continue to have a significantly higher mortality than the general population. HIV status is not associated with an increased risk of infectious complications, however LRTIs are more common in HIV-infected patients.
PO 73

ORAL HPV-RELATED MALIGNANCES IN HIV POSITIVE

INDIVIDUALS: A CLINICAL CASE

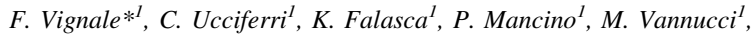
E. Pizzigallo ${ }^{1}$, J. Vecchiet ${ }^{\prime}$

${ }^{I}$ Infectious Disease Clinic, Department of Medicine and Science of Aging " $G$. d'Annunzio" University, Chieti

Introduction: The incidence of human papillomavirus (HPV)-related cancer is increasing among HIV-infected individuals. The two most frequent manifestations of oral HPV infection are HPV-32-associated oral warts and HPV-16-associated oral cancer.

Case report: We describe a clinical case of a Caucasian 48 year-old man, HIV-infected since1995, a drug user, smoker, with chronic hepatitis $\mathrm{C}$, hypertension, previous cryptococcal meningitis and anal and genital condylomatosis. He is a multi-antiretroviral-experienced patient, with poor adherence leading to frequent treatment and followup discontinuations. After one of these episodes, he came to our attention in October 2010 with an oral ulcer on the right cheek which had appeared 15 days before and showed a rapid evolution; histology and immunohistochemistry of biopsy revealed an HPV-16-related epidermoid cancer. The patient had discontinued therapy 6 months before; his viral load was thus 140,000 copies/ml and CD4+ lymphocytes were 11/?L. Total body PET/CT showed an anomalous uptake of the FDG in the right cheek mucosa and in a small right submandibular lymphnode. The patient promptly started cART again with TDF/FTC, DRV/r (800/100 mg) and RAL, chosen on the basis of previous treatment history and a resistance test. The otolaryngologists performed cancer resection, drainage of homolateral lateralcervical lymphnodes and cheek reconstruction with a pedicle flap of the sternocleidomastoid muscle. At the time of surgical intervention, in December 2010, his immune status was slightly improved, while plasma viral load became undetectable. There were no post-surgical complications and the patient was discharged in good clinical condition. The histological examination confirmed a well-differentiated squamous-cell carcinoma, with HPV-16 infection without involvement of lymphnodes and salivary glands.

Discussion: infection-related and cancer-related oral lesions are frequent among HIV-infected individuals; contributing factors, in this population, may be represented by cigarette smoking and alcohol abuse. Their differential diagnosis is essential for a prompt and radical treatment. The incidence of HPV-related lesions does not seem to have decreased with the introduction of HAART. However, immunosuppression may favour progression of pre-cancerous lesions. The evaluation of primary screening and prevention programmes in this population should become a priority.

PO 75

CMV RELATED UVEITIS AND KS IN AN AIDS PRESENTER PATIENT WITH NHL TREATED WITH RITUXIMAB: CASE REPORT

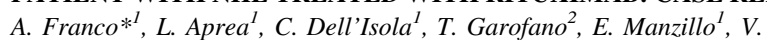
Montesarchio $^{2}$, T. Pizzella ${ }^{l}$, F. Simioli ${ }^{l}$, C.M. Izzo ${ }^{1}$

${ }^{I}$ VIII Department of Infectious Diseases, Monaldi-Cotugno-CTO Hospital, Naples, Italy; ${ }^{2}$ Oncology Unit, Monaldi-Cotugno-CTO Hospital, Naples, Italy

Introduction: Rituximab (MabThera $\odot$, Roche) is a new biological drug used for neoplastic diseases. It is a chimeric monoclonal antibody against CD20+ lymphocytes, and it is used in association with an adequate chemotherapy in the treatment of lymphoid tumor of the B cells line (most NHL and LLC). The mechanism of action consists in an antibody mediated destruction of the CD20+, both tumoral and not, that can cause a prolonged immunodepression; in some patients (e.g. HIV+), it can reactivate chronic silent viral infections.

Case report: OF, 21 years old male from Nigeria, naïve, came to our Department in May 2010, because of multiple lymphadenopathy and recent discovery of anti-HIV positivity; he had a discrete immune 
situation (lymphocytes 2,420/ $\mu 1, \mathrm{CD} 4+433 / \mu \mathrm{l}, \mathrm{CD} 8+1,413 / \mu \mathrm{l}, \mathrm{CD} 4 /$ CD8 0.1), with a HIV viral load of $264,438 \mathrm{cp} / \mathrm{ml}$. During the hospitalization he underwent histhological and instrumental exams: lymphonode biopsy, CT total body, PET/CT, bone marrow biopsy from the iliac creste, lumbar puncture. The final diagnosis was of "High-grade follicular B NHL infiltrating bone marrow and CNS", so he started both HAART (TDF+FTC+LPV/r), systemic chemotherapy (modified R-Chop-14: rituximab $500 \mathrm{mg}+$ cyclophosphamide $850 \mathrm{mg}+$ vincristine $1 \mathrm{mg}+$ liposomal doxorubicine $60 \mathrm{mg}+$ prednisone $75 \mathrm{mg} /$ die for 5 days) and prophylactic treatment to reduce the CNS infiltration (chloridrate cytarabine + betametasone intrathecal). In October 2010, after the sixth cycle of therapy, with an overall administration of $3 \mathrm{~g}$ of rituximab, the patient had a partial remission of the lymphoma (PET/CT improved), but started to loose visual acuity of the left eye. The virological and oculistic exams he underwent (ocular fundus, US of the eyeball, blood CMV DNA), showed a CMV related uveitis with tractional retinal detachment (Figure); because of this he started a specific therapy: intravenous ganciclovir and ocular administration of atropine $1 \%$ and bethametasone/chloramphenicole, with the interruption of the chemotherapy. In November 2010 (after 4 weeks of uveitis onset) we found a lesion of the right foot suggestive for Kaposi's Sarcoma. To date the patient is still under our observation and he experienced a new chemotherapy cycle without rituximab (modified Chop-14).

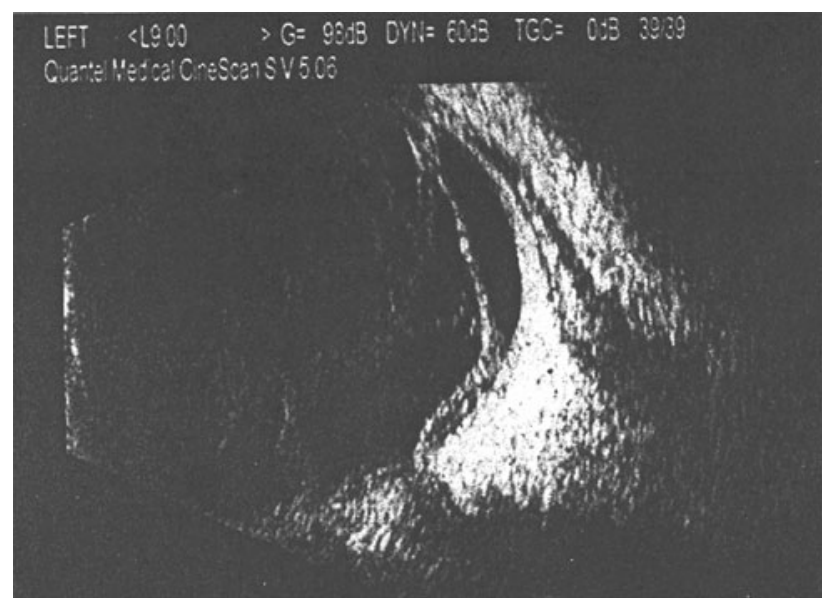

Discussion: The use of rituximab in chemotherapic associations, even if it is correlated to better answers to drugs in high-grade B lymphomas, is not free from risks in HIV+ people, in whom the immunosuppressor agents can cause the reactivation of silent viral infections (CMV, HHV8).

\section{PO 76}

PROTEASE INHIBITORS AND HIV-RELATED DEMYELINATING POLYNEUROPATHIES: A NEW POSSIBLE TREATMENT?

G. Ceccarelli ${ }^{\prime}$, A. Clemenzi ${ }^{2}$, G. Antonini ${ }^{2}$, C. Rizza ${ }^{l}$, G. D'Ettorre ${ }^{1}$, V. Vullo ${ }^{l}$ ${ }^{I}$ Department of Public Health and Infectious Diseases, "Sapienza" University of Rome, Italy; ${ }^{2}$ Department of Neurological Science, II "Sapienza" University of Rome, Rome, Italy

HIV-1 related demyelinating polyneuropathy (HIV-DP) can be triggered by a direct action of the virus itself or be caused by antibodies capable of damaging the myelin sheets, especially when the viremia control is lacking. We present the case report of a HIV-1 infection related inflammatory polyradiculoneuropathy (HIV-PRN), which improved after protease inhibitors (PIs) initiation. In July 2009, a 32-year old heterosexual and drug abuser man was diagnosed HIV-1 infection after the onset of fever, abdominal cramps, and diffuse lymphadenopathy. In September 2009 the CD4+ count was 550 (23\%) and the wild-type HIV-RNA was 119,979 copies/ml. In February 2010, the CD4+ T cell-count showed a reduction to 450 cells/ mmc and a therapy with boosted Darunavir plus Tenofovir/Emtricitabine was prescribed, which the patient refused to take. In April 2010, the patient complained the acute onset of paresthesia/hypoesthesia in the soles of the feet, which within 20 days spread up to the gluteal region, and to the hands up to the wrists, also associated with weakness of the lower limbs. The patient decided himself to start the antiretroviral therapy that had been prescribed before, with subjective improvement in both sensory and motor symptoms after 1 week. The neurological exam performed after an additional week highlighted: strength impairment (MRC scale grade 4) in extensor digitorum, hand's interossei, tibialis anterior and extensor allucis longus muscles of the two sides, both pain hypoesthesia and hypopallesthesia (7/8 on the tuning-fork exam) in the feet up to the ankles and in the hand fingers, areflexia in the lower limbs, and abnormal gait. The ENG examination confirmed a demyelinating PRN. CSF examination showed elevated proteins $(74 \mathrm{mg} / \mathrm{dl})$ with no increase in cell count, 224 copies/ml of HIV-RNA, and negative PCR for both CMV-DNA and other neurotropic viruses. Because of the subjective improvement and the slight neurological signs detected, no immunomodulatory therapy (i.e. IVIg or plasmapheresis) was prescribed and antiretroviral therapy was confirmed (CD4+ 740 (27.57\%), HIV-RNA $<100,000$ copies $/ \mathrm{ml}$ ). The patient was then lost to follow-up. Treatment strategies for HIV-DP (i.e. IVIg and plasmapheresis) are the same as in HIV-negative patients. Despite the satisfactory therapeutic response to these treatments, there are no results available from casecontrol studies. In our patient, however, the regression of the neurological symptoms was associated with the IPs therapy. So far, the risk of neurotoxic effects due to the IPs seems to be very low if compared to its important therapeutic role. However, further studies are needed to establish their potential use in the treatment of HIV-DP.

Acknowledgment: We wish to thank Fiore Crespi and Filippo von Schloesser on behalf of ANLAIDS and NADIR Foundation, respectively.

\section{PO 77}

KAPOSI'S SARCOMA-ASSOCIATED HERPESVIRUS/HUMAN HERPESVIRUS TYPE 8-POSITIVE CUTANEOUS LYMPHOMAS S. Migliore ${ }^{\prime}$, N. Storaci ${ }^{l}$, G. Gambuzza ${ }^{l}$, A. Famá ${ }^{l}$, A. Iacono ${ }^{l}$

${ }^{I}$ UOC Malattie Infettive, Ragusa, Italy

Kaposi's sarcoma (KS) is one of the most common opportunistic diseases associated with HIV infection, and remains the most frequent malignancy in his setting [1,2]. Human herpes virus 8 (HHV-8) is now recognized as the causative agent [3]. Kaposi's sarcoma-associated herpesvirus (KSHV) is a tumor consisting of both capillaries and fibrosarcoma-like cells. This lesion predominates in men and occurs commonly in patients with acquired immunodeficiency syndrome (AIDS). We present a case of naïve HIV - HCV positive man with cutaneous lymphoma associated KSHV and HHV8. He presented extranodal masses in the skin, on the right thigh, that measuring $\sim 2 \mathrm{~cm}$ in diameter. The gastrointestinal tract was not involved. The patient did not develop a lymphomatous effusion. Epidemiological and clinical features are summarized in Table 1. Table 1

\begin{tabular}{ll}
\hline Age/sex & $49 / \mathrm{M}$ \\
\hline HIV-serology (risk group) & Positive (heterosexual) \\
HIV disease & $\mathrm{CD} \mathrm{3}$ \\
KSHV-related disease & $\mathrm{KS}$ \\
$\mathrm{CD} 4$ cell count $/ \mu \mathrm{L}$ & 25 \\
\hline
\end{tabular}


Table 1 continued

\begin{tabular}{ll}
\hline Age/sex & $49 / \mathrm{M}$ \\
\hline HIV-RNA cp/mL & 161.000 \\
HCV-RNAUI/ml & 45,600 (genotype 1b) \\
ALT U/I $(<35)$ & 65 \\
PS (ECOG) & 4 \\
Stage (A. Arbor) & IVB \\
B-symptoms & High fever, weight \\
& loss \\
Site of involvement & Skin \\
Major laboratory abnormalities: & \\
Severe anemia $(\leq 8.0 \mathrm{~g} / \%)$ & Present \\
Thrombocytopenia $(\leq 100,000 / \mu \mathrm{L})$ & Present \\
Coomb's test & Positive \\
Abnormal LDH serum level $(\geq 460 \mathrm{U} /$ & Present \\
mL) & \\
Hypoalbuminemia $(\leq 3 \mathrm{~g} / \%)$ & Present \\
Therapy & CHOPLD $\dagger$ \\
Outcome & Living
\end{tabular}

We performed the skin biopsy. The diagnosis was "location dermo-hypodermic large cell lymphoma with phenotype anaplastic, associated infection KSHV-HHV8". Histologically, it is characterized by a proliferation of spindle cells and cleft-like vascular structures with vascular channel formation (Fig. 1). The case showed greater pleomorphism and a broader range of cell size and anaplastic features. It is the most common neoplasm occurring in HIV positive patients. Immunohistological analysis revealed that the patient was positive for CD3, CD138, MUM1/RF4, ORF73/HHV8+, CD30 and negative for $\mathrm{C} 20, \mathrm{CD} 45$ and CD34.
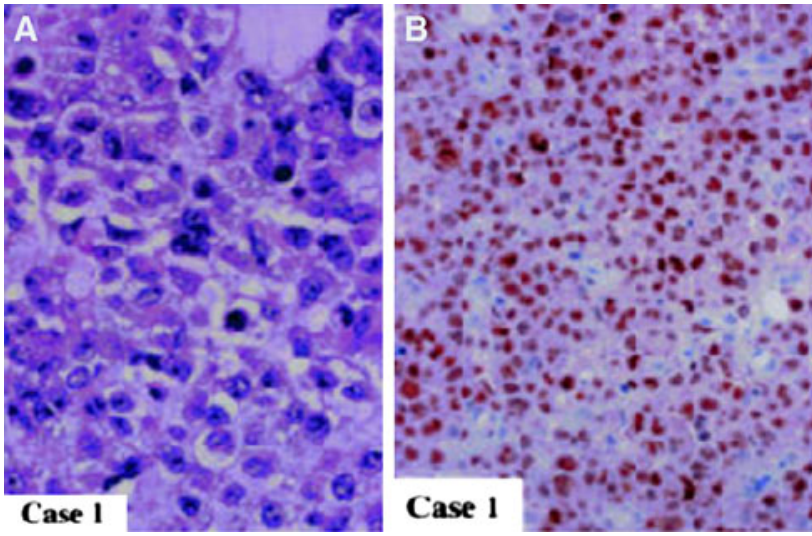

The dual-phase CT scan showed no lesions, and nuclear medicine studies with F-FDG, showed overactive area to the right thigh. $\mathrm{He}$ received antiretroviral therapy [4] with emtricitabine-tenofovir disoproxil fumarate, efavirenz and raltegravir, obtaining the negativity of viral load in 1 month, and then began chemotherapy (CHOPLD $\dagger$ ), obtaining the remission of lymphoma associated KSHV and HHV8. Conclusions: The early diagnosis of HIV and lymphoma associated KSHV - HHV8 allowed the beginning of antiretroviral therapy and chemotherapy obtaining the regression of the tumor under.

\section{PO 78}

SEVERE OSTEOPOROSIS IN A YOUNG HIV-INFECTED MAN TREATED WITH TERIPATIDE

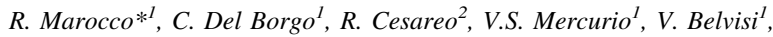
M. Lichtner ${ }^{l}$, C.M. Mastroianni ${ }^{1}$

${ }^{I}$ Infectious Disease Unit, SM Goretti Hospital Latina, Italy; ${ }^{2}$ Endocrinology, SM Goretti Latina, Latina, Italy

Background: Osteoporosis is recognized as one of secondary cause of HIV-associated bone disorders, but multiple vertebral osteoporotic fractures are rarely reported in HIV-infected patients. Standard treatments for osteoporosis are not defined in HIV population.

Case report: We report a case of HIV+ 37 years old man presenting with low back pain in our HIV day-center. HIV infection has been diagnosed 9 years earlier. He was taking antiretroviral therapy (ARV) with lopinavir-ritonavir, zidovudine and lamivudine. Nadir CD4 T-cell counts was 248 cells/mmc and HIV viral load zenith was $112,000 \mathrm{c} / \mathrm{ml}$. Complete virological suppression and immunological recovery (CD4 cells count $465 / \mathrm{mmc}$.) was observed after 3 months of ARV. One month later the patient developed neurologic syndrome characterized by fever and right hemiparesis defined as IRIS treated with prednisone $40 \mathrm{mg} / \mathrm{die}$ with subsequent tapering reduction until $5 \mathrm{mg}$. Complete neurologic recovery was observed. After 2 months the beginning of therapy with steroids he developed low back pain. A RMN and CT of dorsal and lumbar tract documented multiple vertebral fractures with radiologic evidence of severe osteoporosis. Steroid therapy was stopped and ARV was continued. Dexa examination documented severe osteoporosis in lumbar zone T score of ($33 \%)-1.66$ and $\mathrm{Z}$ score of $(-33 \%)-1.66$. All analysis excluded the presence of evident causes of secondary osteoporosis in particular hypogonadism. Calcemia value was normal. The patient refused bone vertebral biopsy and vertebroplastic surgical intervention. Therapy with alendronate was started. The low back pain persisted and after 3 months CT and RMN documented no improvement of radiological picture. Considering the severity and the presence of multiple vertebral fractures, teripatide was started and administered at the dose of $20 \mu \mathrm{g} / \mathrm{die} / \mathrm{s} . c$. After 2 months the patients reported reduction of low back pain. Teripatide was administered for 18 months. Control of Dexa documented in lumbar zone $\mathrm{T}$ score of $(-38 \%)-1.93$ and $\mathrm{Z}$ score of $(-37 \%)-1.92$. Control of RMN documented reduction of edema of spongiosa and didn'find any new fractures. The patient did not report pain and other new fractures during other 1 year of followup.

Conclusion: Osteoporosis and osteopenia is an emerging problem in HIV population associated to use of ARV and to HIV itself. In our case severe osteoporosis was observed in a very young patient without significant risk factors except for corticosteroid use and very short exposition to ARV therapy. Standardized screening for osteopenia and osteoporosis is suitable for all HIV infected patients after the diagnosis or before starting ART to prevent bone fractures and reduce risk factors exposition. Teripatide may represent a potential and useful agent for management of severe HIV-associated osteoporosis.

\section{PO 79}

PROGRESSIVE DISSEMINATED HISTOPLASMOSIS DETECTED BY A RAPID AND AGGRESSIVE DIAGNOSTIC APPROACH

G. Montorzi ${ }^{\prime}{ }^{1}$, L. Zammarchi ${ }^{I}$, J. Nocentini ${ }^{I}$, F. Baragli ${ }^{I}$, D. Magnelli ${ }^{I}$, L. Attala ${ }^{1}$, A. Bartoloni ${ }^{l}$

Clinica Malattie Infettive, Università di Firenze, Florence, Italy

Progressive Disseminated Histoplasmosis (PDH) is an AIDS defining opportunistic infection rarely reported in Italy, mostly in migrants but with few autochthonous cases as well. The diagnosis is challenging because the disease is rare and the urinary specific test is unavailable in Europe. Currently in Europe the diagnosis can be 
achieved by histopathological exam or culture isolation performed only in biosafety level 3 laboratory (Antinori 2006). A 35 years old Ecuadorian man, in Italy since 7 years, admitted the 20/12/2010 with a 1 month history of high fever, weight loss and dry cough resistant to oral antibiotics. Past clinical history was unremarkable and physical examination showed nothing relevant, except for fever $\left(39^{\circ} \mathrm{C}\right)$. At blood exams elevated transaminases (AST $196 \mathrm{UI} / \mathrm{mL}$, ALT $129 \mathrm{UI} / \mathrm{mL})$, high LDH $(1,681 \mathrm{UI} / \mathrm{mL})$, CRP $(108 \mathrm{mg} / \mathrm{dL})$, ESR $(51 \mathrm{~mm} / \mathrm{h})$, ferritin $(>16,000 \mathrm{ng} / \mathrm{mL})$. Normal blood gas analysis. Chest X-ray showed a paracardiac right opacity better studied by chest CT-scan with evidence of medium lobe pseudonodular infiltrate, bilateral ground glass areas and $3 \mathrm{~cm}$ mediastinum colliquated adenopathies. At abdominal ultrasound presence of $13.5 \mathrm{~cm}$ splenomegaly, confirmed by abdominal CT scan. HIV1-2 Ag-Ab screening test was positive. HIV1 RNA was $3 \times 10^{6} \mathrm{UI} / \mathrm{mL}$ with a CD4 count of $31 / \mathrm{mm}^{3}-8.2 \%$; HBV markers negatives; HCV Ab and viremia negatives; previous infection by CMV, EBV, HSV1, T. gondii; TPHA 1:640, RPR 1:1. Quantiferon and TTs negatives, three sputum samples negatives for mycobacteria. Six samples of blood colture negatives, ongoing the mycobacterial ones. CMV PCR was $22,000 \mathrm{cp} / \mathrm{mL}$. The $22 / 12$ empirical first line antitubercular treatment was started but stopped 2 days later for toxic hepatitis. Because of persistent high fever, the 27/12 empirical iv therapy for both $M$. tuberculosis and atypical mycobacteria with amikacin, ethambuthol and rifabutin was started without improvement. More aggressive diagnostic tests were performed: the 30/12 bone marrow and liver biopsy, the 31/12 bronchoscopy, blood samples collected for Leishmania PCR, Aspergillus galactomannan (AGM) assay, $H$. capsulatum and T. cruzii serology, blood slides for malaria. Because of surprising positivity of AGM (DO 4.49), the 4/01 liposomal amphotericin B $4 \mathrm{mg} / \mathrm{kg} /$ day was started, being the less interactive drug with PI-based HAART prescribed the same day. Again surprisingly the 10/01 histological exam on liver and bone marrow samples resulted positive for $H$. capsulatum, even if the serology was negative. Therapy was confirmed, the $9 / 01$ patient became afebrile with clinical recovery and normalization of blood values. This case highlights the need of considering PDH in the differential diagnosis of fever of unknown origin in AIDS mostly in patients from endemic area and the importance of a rapid aggressive approach in order to avoid fatal outcome (Faggi 2001). False positive results of AGM in patients with Histoplasmosis have already been reported (Nareddy 2007).

\section{PO 80}

\section{AVASCULAR NECROSIS OF THE BONE IN HIV-INFECTED} PATIENTS: A POSSIBLE RULE OF IMMUNE RECONSTITUTION INFLAMMATORY SYNDROME

F. Prati ${ }^{l}$, E. Barchi ${ }^{*}$, G. Zoboli ${ }^{l}$, G. Magnani ${ }^{I}$

${ }^{I}$ U.O. Malattie Infettive ASMN, Reggio Emilia, Italy

Introduction: Avascular necrosis (AVN) is increasingly recognized as a complication of HIV infection during the era of HAART with incidence higher than 50 times the rate expected in the general population. AVN results from poor arterial blood supply to the bone and it has been related to both the use of proteasis inhibitor (PI) and to HIV-1 infection itself, along with traditional risk factors (corticosteroid use, alcohol abuse, smoking, hyperlipidemia, osteoporosis, coagulopathies)

Case reports: Three cases of AVN were identified from 2007 to 2010 in HIV-infected outpatients attending our HIV Clinic in Reggio Emilia. Patient 1, 33-year-old man, stage C3, ANV of the right tibia, presenting the following risk factors: short-course steroid (given for the treatment of Pneumocystis jeroveci pneumoniae) and moderate hypertriglicerid. Patient 2, 47-year-old man, stage B2, AVN of the right femoral head, with risk factors from diabetes mellitus and BMI $<19 \mathrm{~kg} / \mathrm{m}^{2}$. Patient 3, 56-year-old woman, stage B3, presented with bilateral femoral necrosis, and severe osteoporosis as a predisposing factor. Other risk factors were not present.Two of the above patients presented HIV viral-load $>1,000,000$ copies $/ \mathrm{ml}$ at the moment of HIV diagnosis; HAART was based on PI/r + NRTI on all three patients. ANV was diagnosed 8 months after the beginning of HAART (range 5-12 months), by that time all the patient reached a good immunological recovery $(14 \%$ mean increasing in the CD4 count from the beginning of HAART) e complete virological suppression. ANV diagnosis was driven by the presence of pain syntoms in the area of the joint and later confirmed by MNR examination. All patients went through hyperbaric oxygen therapy and reached regression of the pain sinthomatology. Stabilization of necrosys (FOCI) took place on all but case 1, where instead it was witnessed a worsening during the first year involving multiple areas (femur, shoulder, heel, contralateral tibia), later followed by stabilization and partial improvement. Case 1 received even pulsed magneto therapy; case 1 and 3 were treated with bisphosphonates.

Discussion: Clinical suspicion and early detection (thanks to RMN, the most sensitive diagnostic tool) remains the best prognostic factor for a successful medical treatment of osteonecrosis, regardless of causes. As reported in some case-studies, ANV may appear even after a few moths from the beginning of HAART typically associated with a good virological and immunological response. As this cases suggest, immune reconstitution inflammatory syndrome may have a role in the pathogenesis of the AVN.

\section{PO 81}

CLINICAL SIGNIFICANCE AND MANAGEMENT OF SEVERE HYPONATREMIA IN A CRITICALLY ILL AIDS PRESENTER

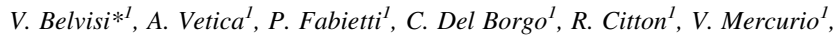
T. Tieghi ${ }^{1}$, C.M. Mastroianni ${ }^{l}$

${ }^{I}$ Infectious Disease Unit, SM Goretti Hospital Sapienza University, Latina, Italy

Symptoms of hyponatremia are largely due to CNS dysfunction and more evident when decrease in the serum $\mathrm{Na}+$ concentration is large or rapid. This case report shows the impact of this electrolyte imbalance in the context of comorbidities affecting a newly diagnosed AIDS presenter. A 29-years-old homosexual Brazilian man presented to Emergency Department complaining of headache and vomiting and weight loss. Vital parameters and blood tests were all normal except for $\mathrm{Na}+(117 \mathrm{mmol} / \mathrm{L})$. He presented multiple bluish nodules on skin and soft palate alike these of Kaposi's Sarcoma. On neurologic examination the patient was oriented, but shows muscle weakness on right arm and leg. A CT scan of the brain shows a lesion of $40 \mathrm{~mm}$ in the right parietal lobe with perilesional edema and a left periventricular mass of about $18 \mathrm{~mm}$. HIV test required in emergency was positive. CD4+ count was $36 / \mathrm{mmc}(5 \%)$ and HIV-RNA 91,390 copies/mL. Examination of CSF revealed 43 cells $/ \mathrm{mmc}$. Cryptococcus antigen, search for mycobacteria were negative. PCR for T. gondii was positive, neurotropic virus, Mycobacteria and Treponema were all negative. The HIV-RNA level in CSF was 28,740 copies/m. TPHA was positive $(1: 20,480)$. An EGDS showed bluish-red vegetations in gastric antrum and in the second duodenal portion suggesting for KS. A total body CT demonstrated on chest scan a consolidation in left lower lobe; no abnormalities on abdominal scan. Fluid and electrolyte replenishment, anti-edema and anti-toxoplasma therapy (cotrimoxazole) and azithromycin (prophylaxis anti MAC) were started since the admission, but soon he developed leukopenia and cotrimoxazole is replaced by clindamycin. An attempt to start ART with LPV/r, 3TC+AZT, was stopped after 6 days because 
of hyponatremia, vomit, diarrhoea, increase lipase (1,686 IU/L) and amylase (78 IU/L). Total parenteral nutrition was established and was switched to enteral nutrition with nasogastric tube; then general conditions slightly improved. Treatment with ganciclovir was performed on the basis of CMV viral load $(150,000$ copies $/ \mathrm{mL})$ and rectal swab positive for CMV. Clinical conditions deteriorated, despite gradual introduction of ARV-based regimen with DRV, MVC, 3TC/ZDV. The patient developed: severe hyponatremia (98 mmol/L) with agitation, drowsiness, basal pulmonary rales; leukopenia (WBC 1,090/mmc); acute pancreatitis (amylase 1,708 IU/L). Untreatable hyponatremia and hypotension suggested an acute adrenal insufficiency, maybe due to CMV or mycobacteria adrenalitis. Therapy with glucocorticoids at high doses was started but patient died at day 34 from admission. In an AIDS presenter, the simultaneous occurrence of more than one opportunistic infection or complication can explain a single clinical confounding parameter (e.g. causes of hyponatremia: vomiting for brain mass; vomiting and diarrhea for pancreatitis; adrenal acute insufficiency) and its management can be extremely difficult.

\section{PO 82}

THE MIGRATION PROJECT IN VENETO REGION: EVALUATION OF THE EPIDEMIOLOGICAL IMPACT OF TUBERCULOSIS, HIV, HBV, HCV INFECTIONS AND SYPHILIS (MARCO POLO PROJECT)

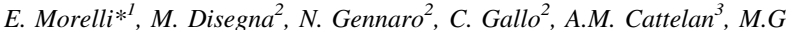

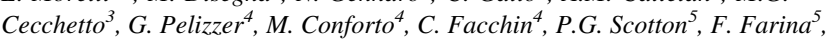

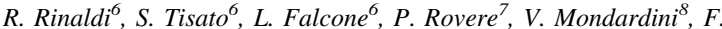

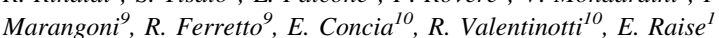

${ }^{I}$ U.O.di Malattie Infettive, Ospedale SS Giovanni e Paolo, Venezia e Ospedale dell'Angelo, Zelarino (Mestre), Italy; ${ }^{2}$ Agenzia Regionale Socio Sanitaria del Veneto, Venezia, Italy; ${ }^{3}$ U.O.di Malattie Infettive, Ospedale Santa Maria della Misericordia, Rovigo, Italy; ${ }^{4}$ U.O. di Malattie Infettive, Ospedale San Bortolo, Vicenza, Italy; ${ }^{5}$ U.O.di Malattie Infettive, Ospedale Ca' Foncello, Treviso, Italy; ${ }^{6}$ U.O.di Malattie Infettive, Azienda Ospedaliera di Padova, Padua, Italy; ${ }^{7}$ U.O.di Malattie Infettive, Ospedale di Legnago, Italy; ${ }^{8}$ U.O.di Malattie Infettive, Ospedale San Martino, Belluno, Italy; ${ }^{9}$ U.O.di Malattie Infettive, Ospedale di Schio; ${ }^{10}$ U.O.di Malattie Infettive, Azienda Ospedaliera di Verona, Italy

Objectives: The immigration phenomenon, as it has been registered in Italy in the last 10 years, has strongly affected the epidemiology of infectious diseases. This study aimed at: conducting an epidemiological survey on the prevalence of HIV, HBV, HCV infections, Syphilis and Tuberculosis (TB) in the immigrant population; evaluating the economical impact of appropriate prophylaxis and therapeutic strategies on the immigrant population in an area of northern Italy (Veneto Region).

Methods: This survey is a multicentric-observational prospective study which involved nine Health Centres in Veneto Region. In order to guarantee a representative sample of the immigrants living in Veneto Region, each centre was asked to recruit a specific number of patients stratified according to their country of origin and their legal status (with or without a regular visa) in Italy. All patients aged $>18$ years and having access to care in the ER unit of each Centre for whatever health problems were invited to participate in the study. The patients enrolled were screened for HIV, HBV, HCV, Syphilis and Tuberculosis by blood tests. Moreover, all patients enrolled filled in a questionnaire in order to asses: demographic and social profile, sexual behaviour, of the immigrants tested. The patients were enrolled followed written informed consent.

Results: From March 2009 to December 2010 a total of 340 immigrant patients has been enrolled in the study, $160(47 \%)$ were male and $180(53 \%)$ female. The 25-34 year age group was the more rappresentative $(35.5 \%)$. Of the 340 patients enrolled, most $(47.3 \%)$ came from South America and Southeast Asia, 36.1\% from East Europa, $15.2 \%$ from Sub-Saharan Africa, only 4 patients were from West Europe. 8.5 (29)\% of these were irregular immigrants. Of the 340 patients enrolled only $3(0.8 \%)$ resulted HIV infected positive, 9 (2.6\%) were HBsAg positive, 9 (2.6\%) had a positive result for Hepatitis C antibody test while 8 (2.3\%) for VDRL test. $25.6 \%$ (87) of the patients enrolled had a positive result for Quantiferon-TB test. The socio-demographic characteristics and anamnestic data on tuberculosis of these patients were analysed. A positive result of this test suggests that $M$. tuberculosis infection is likely. In these subjects active TB has to be excluded and treatment for Latent TB has to be considered.

Conclusions: These preliminary results show that the prevalence of sexual transmitted diseases among immigrant patients is relatively low, respectively $0.8 \%$ for HIV infection, about $3 \%$ for HBV infection and for $\mathrm{HCV}$ infection, roughly $2 \%$ for syphilis, while we found an high prevalence of people with positive Quantiferon-TB test $(25.6 \%)$. That suggests the importance to define and implement, as public health measure, appropriate and effective TB screening programs in the immigrant population, especially in people coming from low-income countries where TB prevalence is high, in order to control the TB transmission and prevent the rebound of TB in the developed countries.

\section{EPIDEMIOLOGY AND SOCIAL SCIENCES}

\section{PO 83}

HIV PREVALENCE AMONG PATIENTS WITH SEXUALLY TRANSMITTED INFECTIONS IN ITALY 1991-2007

V. Regine ${ }^{* 1}$, M.C. Salfa ${ }^{1}$, M. Raimondo ${ }^{1}$, L. Camoni ${ }^{1}$, M. Giuliani ${ }^{1}$, B. Suligoi ${ }^{1}$ and the Italian STI Surveillance Working Group ${ }^{2}$

${ }^{1}$ Centro Operativo AIDS, Istituto Superiore di Sanita, Rome, Italy; ${ }^{2}$ G. Angelini (Bari), N. Aste (Cagliari), M. Cusini (Milano), A. D'Antuono (Bologna), S. Delmonte (Torino), A. Di Carlo (Roma), I. El-Hamad (Brescia), A. Matteelli (Brescia), G. Moise (Gorizia), L. Priano (Genova), F. Urbani (Trento), G. Zuccati (Firenze)

Objectives: To estimate the prevalence of HIV infection among patients with Sexually Transmitted Infections (STl), to assess socio-demographic and behavioral characteristics of HIV-positive patients, and to evaluate the proportion of undiagnosed HIV-positive persons.

Methods: Data were obtained from the Italian STI Sentinel Surveillance System (1991-2007). This System collects information on STI diagnoses provided by a network of 12 public STI clinics located in main cities.

Results: Between 1991 and 2007, 51,765 patients diagnosed with a STI underwent HIV testing. The overall HIV prevalence was $7.6 \%$ [95\% Confidence Interval (CI) 7.3-7.9]. Among the 3,927 HIVpositive patients, $28.3 \%$ were undiagnosed for HIV. HIV-positive patients had a mean age of 34.2 years (standard deviation \pm 8.6 years); more than half of them were males $(76.2 \%) ; 14.7 \%$ were non-nationals; $46.9 \%$ were men having sex with men (MSM); injecting drug use was reported by $34.1 \%$ cases; $60.0 \%$ reported having had two or more partners in the previous 6 months; a previous STI was reported by $50.0 \%$ cases. When analyzing by STI diagnosis, HIV prevalence was $15.0 \%$ among patients with primary or secondary syphilis, $10.5 \%$ among patients with genital herpes, $9.6 \%$ among patients with genital warts, $7.3 \%$ among patients with gonorrhea, $4.0 \%$ among patients with non-gonococcal/non-chlamydial infections, and $2.3 \%$ among patients with chlamydia infection. At the multivariate analysis, HIV prevalence was significantly associated with injecting drug use (OR 41.5; 95\% CI 36.7-46.8), MSM (OR 9.2; 95\% 
CI 8.3-10.3), reporting a previous STI (OR 2.7; 95\% CI 2.4-2.9), and age older than 26 years (OR 2.2; 95\% CI 1.9-2.5).

Conclusions: The data from the Italian STI Sentinel Surveillance System show that HIV prevalence among STI patients is higher than that reported by other European STI surveillance systems, such as UK and the Netherlands (McGarrigle CA et al., Sex Transm Infect 1998; Op de Coul ELM et al., Euro Surveill 2006). HIV infection circulates more frequently among persons with an ulcerative STI or genital warts. The relevant proportion of STI patients with undiagnosed HIV infection stresses the need for an active proposal of HIV testing (including the opt-out approach) among persons with a STI.

\section{PO 84}

\section{HIV INFECTION IN A COHORT OF ADULT MIGRANTS}

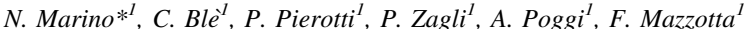

${ }^{1}$ Azienda Sanitaria Firenze, Florence, Italy

Background: Migrations are a phenomenon of world-wide dimension with the obvious political, social and economic implications. Italy, due to its geographical location, is one of the countries more affected by this problem in the world: in 2010, the foreign residents were almost 5 millions (1 immigrant every 12 residents) - XX Immigration Report of 2010-Caritas Migranteswith 3 million of migrants in the course of the last 10 years, and nearly 1 million during the last 2 years. More than $62 \%$ resides in Northern Regions of Italy; 25\% in Central Regions and 13\% live in the South. In between 1985 and 2008 in Italy 42.747 new HIV diagnosis have been performed (ISS-COA 2010); the proportion of migrants among the new diagnoses of HIV infection increased from $11 \%$ in 1992 to $31.6 \%$ in 2008 ; incidence and prevalence rates are significantly higher in migrants than in the native population: almost $20 \%$ of the AIDS cases in Italy is taking place in migrant population, and the incidence of new HIV infections in this population is equal to $60 / 100,000$, nine times more than the local population. Moreover almost $70 \%$ of the migrants with an AIDS diagnosis have discovered HIV positivity just in the previous 6 months.

Methods: To determine HIV prevalence, consecutive adult migrants in the years 2000-2010 were tested for antibodies against HIV types 1 and 2. Epidemiological, clinical and therapeutical data were collected. $t$ Student test was employed.

Results: A total of 2,930 migrants $(71.3 \%$ males, median age 34.3 years, $37 \%$ from East Europe, $24.5 \%$ from Africa, $38.5 \%$ from Orient) were evaluated: $332(11.33 \%)$ were HIV positive, $532(18 \%)$ HbsAg positive, 177 (6\%) HCV positive. Migrants HIV positive versus Italian HIV positive were: male 76 versus $71 \%$, mean age 35 versus $39 \%$, HCV coinfected 2 versus $44 \%$, HBV coinfected 4 versus $5 \%$, heterosexual transmission 51 versus $15 \%$, CD4 nadir $<20039$ versus $24 \%$, on HAART 11 versus $44 \%$.

Conclusions: The management of HIV infection in the migrants has the same indications of the native subject but it must exceed the linguistic-cultural differences and the difficult access to the healthservices in order to guarantee the adherence. The short time in between HIV positivity and AIDS diagnosis is related to several factors, like lack of proper information, the fear to face the disease, and we have to consider the peculiar condition of this population with particular regard to level of poor education and shortage of economical resources, traditional believes and religious issues.

\section{PO 85}

SURVEILLANCE SYSTEM FOR HIV INFECTION IN TUSCANY

V. Casotto ${ }^{*}$, F. Voller $^{I}$, E. Balocchini ${ }^{2}$, F. Cipriani $^{1}$ Regional Health Agency of Tuscany, Florence, Italy; ${ }^{2}$ Tuscany Region, Florence, Italy

Introduction: Since 1985, AIDS cases diagnosed in health facilities throughout Tuscany are compulsorily reported to the regional AIDS registry. Today the surveillance of AIDS cases essentially gives indications on the accessibility and effectiveness of secondary prevention services but it is rather inadequate for monitoring the epidemic of acquired human immunodeficiency virus (HIV) infections. So, it is necessary to implement systems for monitoring HIV infection in order to identify new groups with the highest personal and behavioural risk and steer the activities of prevention. The Italian Ministry of Health-Decree of March 31 2008-has promoted the activation of the monitoring system of new diagnoses of HIV infection in Italy. The surveillance system of HIV diagnoses in Tuscany started in May 2011 and it is currently being implemented.

Objective: The surveillance system of HIV infection has the following objectives: (1) to define the incidence of newly diagnosed HIV cases; (2) to estimate the prevalence of HIV infection; (3) to provide useful data for defining the demand for health services for the care of people with HIV; (4) to determine risk factors for progression of HIV infection to AIDS; (5) to plan and evaluate preventive measures.

Methods: Regional surveillance for newly diagnosed HIV infection was based on cases of infection reported by structures enabled (infectious diseases units and sexually transmitted diseases unit) by the regional HIV surveillance center (Unit of Epidemiology, Regional Health Agency of Tuscany) on the first occasion of diagnosis in Tuscany. Data are collected by filling out a special regional notification form, and the regional HIV surveillance center provides for the computerization of forms received from the reporting units in a data base. Results: Preliminary data on the diagnosis of HIV infection in Tuscany will be available in the first half of 2011. However, there are currently some information on the HIV-positive individuals in care at the units of infectious diseases for the period January-September 2008. During this period, 4,457 HIV-positive patients were followed by the infectious diseases unit. Of these cases, $24.2 \%$ was female and most of patients aged between 35 and 59 years at diagnosis $(61.6 \%)$. Women were younger than males: $33.4 \%$ aged between 17 and 34 years compared to $25.9 \%$ of males. Exposure to HIV for the majority $(70 \%)$ of patients was sexual contact: $26.8 \%$ homosexual contact and $43.2 \%$ heterosexual contact. Of the 4,457 HIV-positive patients $14.8 \%$ (658 patients) were diagnosed with AIDS, while for the 183 subjects this information is not known. With regard to HIVpositive patients not yet on AIDS $(3,616)$, most of them $(90.4 \%)$ had a CD4+ T-lymphocyte count greater than or equal to 200 .

Conclusions: The monitoring system of new diagnoses of HIV infection is the best way to describe the changes of the epidemic and provide the tools needed to plan interventions for primary and secondary prevention. 


\section{PO 86}

20-YEAR (1990-2009) CHANGING PATTERN OF PERSONS

ATTENDING AT ITALIAN NATIONAL INSTITUTE FOR

INFECTIOUS DISEASES (INMI) "LAZZARO SPALLANZANI" FOR HIV COUNSELLING AND TESTING IN ROME

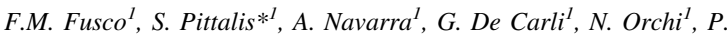

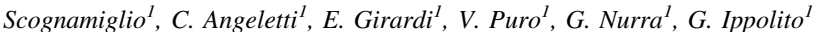

${ }^{I}$ Istituto Nazionale per le Malattie Infettive "Lazzaro Spallanzani", Rome, Italy

Background: In Italy, since 1985, confidential and voluntary HIV counselling and testing (CT) can be obtained free of charge and, when requested, anonymously, in publicly funded sites by person selfreferral (SR) or referred by a physician (PR). The HIV-CT site of the Italian National Institute for Infectious Diseases "L. Spallanzani", Rome, is one of the largest in Italy and represents the regional referral centre.

Methods: In order to evaluate if reasons (not risk factors) for attending CT site and demographic characteristics vary over time, we retrospectively analyse data from 29,326 adults (54\% women) receiving first lifetime HIV-CT divided into 5-year period each (1990-1994, 1995-1999, 2000-2004, 2005-2009).

Results: The number of persons seeking first lifetime HIV-CT progressively decreases $(8,786,8,311,5,840,6,384)$. The average age progressively increases $(32,33,34,36$ years, overall median 31$)$, as the proportion of foreign persons $(6,8,15,17 \%)$. About reasons for testing, heterosexual intercourse with partner of unknown serostatus (Het), that is the first motivation in 1990-1999 (36\%), significantly decreases in 2000-2009 (26\%), when the first reason becomes pregnancy/family planning (Preg). The proportion of persons stating the use of injecting drugs (ID) as reason for HIV-CT decreases considerably $(3,1.5,0.6,0.4 \%)$, while an upward trend emerges in the proportion of persons who require HIV-CT because of clinical symptoms considered as HIV-related $(6,8,9,11 \%)$. Other main reasons for testing, i.e. sexual intercourse with a known HIV+ partner $(\mathrm{SP}+)$ and homosexual intercourse with partner of unknown serostatus (Hom), remain stable. Among those PR, main reasons are Preg and suggestive symptoms, while sexual intercourse is the main reason among SR. Overall, HIV prevalence is $3.2 \%$ in men, $1.4 \%$ in women: no significant time trend is noted. HIV prevalence do not vary among foreign persons (5.1 overall). The highest prevalence is among SP+ (14.9 overall) and Hom (11.3 overall; 9.4 in 2005-2009). HIV prevalence among Het decreases $(1.2,0.9,1.2,0.6)$, as Preg $(1.9,0.4$, $0.3,0.3$ ) and ID (12 in 1990-1994, 0 in 2005-2009); conversely, HIV prevalence increases in persons who require HIV-CT for symptoms (3.2, 2.6, 7.4, 5.2), both in SR and PR

Conclusions: our data highlights a changing pattern of persons attending CT site. The decrease of proportion of Het is in contrast with the increase of HIV-positivity among heterosexuals in Italy, and suggests that risk awareness among this population is decreasing. The increase of persons who require HIV-CT for symptoms may indicate an improved awareness of physicians and patients, or a higher proportion of late presenters. The advances in prevention, treatment and care of HIV infection support the need to optimise HIV CT programs: a better knowledge about reasons for testing should be used to develop targeted counselling and testing prevention strategies.
PO 87

RESULTS OF EPIDEMIOLOGICAL SURVEILLANCE OF AIDS CASES IN TUSCANY

J.J.V. Branca $*^{2}$, S. Pacini ${ }^{2}$, M. Ruggiero ${ }^{3}$

${ }^{2}$ Department of Anatomy, Histology and Forensic Medicine, University of Firenze, Florence, Italy; ${ }^{3}$ Department of Experimental Pathology and Oncology, University of Firenze, Florence, Italy

The Regional Public Health System of Tuscany recently achieved remarkable successes in fighting AIDS with fewer than 10 deaths per year of diagnosis in 2008 and 2009 in a population of almost 4 million residents. Of the many factors that contributed to this success, here we shall focus on results obtained by accurate epidemiological surveillance conducted by the Regional Agency for Health (Agenzia Regionale di Sanità). It is worth noting that a regional registry of new HIV infections is not yet available; therefore the data reported here refer only to new AIDS cases. However, the very low numbers of AIDS deaths ( 2 in 2008 and 7 in 2009) seem to indicate that surveillance of new AIDS cases is probably the most effective epidemiological tool in fighting AIDS. In fact, data from the regional registry of new AIDS cases reveals that the male-to-female ratio for the incidence of AIDS has been essentially constant from 1985 to 2008 at $\sim 3.6$, whereas the purported mode of transmission changed drastically: from $\sim 8 \%$ of HIV being transmitted heterosexually in 1985-1990, to 44\% being transmitted in that way in 2006-2008. Consistent with these data, in recent years (updated to 2009), 44.5\% HIV-positive heterosexuals reported being aware of their serostatus before the diagnosis of AIDS, and $17.8 \%$ had been treated with antiretroviral drugs prior to the diagnosis of AIDS. Conversely, about $90 \%$ HIV-positive intravenous drug users reported knowing their serostatus before the diagnosis of AIDS, and $57 \%$ of them were treated with antiretroviral drugs before the diagnosis of AIDS. Regrettably, however, the probability of survival in the HIVpositive intravenous drug user population has been constantly lower than that observed in the other categories since 1996. Drugassociated multi-organ toxicity might have contributed to this phenomenon. Also the age trend for new AIDS cases shows interesting changes that might have contributed to the decline in AIDS mortality in Tuscany. In 1988, the mean age for new AIDS cases was 31 for males and 28 for females, whereas in 2009 the mean ages were 44.5 and 40 , respectively. We believe that information strategies focussed on risky sexual behaviours might have contributed to this trend. In fact, a recent survey of teenagers' sexual behaviour (Indagine EDIT 2008) showed that $43.5 \%$ of teenagers had had actual sexual intercourse and only $37.9 \%$ (males) and $26.4 \%$ (females) teenagers reported more than three partners. Among female teenagers reporting more than three partners, $45 \%$ reported using condoms. Taken together, these most recent data are consistent with previous observations on AIDS epidemiology (Ital J Anat Embryol. 2009 Apr-Sep;114 (2-3): 97-108. Oct-Dec;114 (4):179-91), and may suggest that further improvement in AIDS patient survival could be achieved by focussing efforts on reducing or eliminating drug-associated toxicity. 


\section{PO 88}

\section{HIV/AIDS: UNA MALATTIA DIMENTICATA}

S. Corso ${ }^{* 1}$, A. Poggi $i^{2}$ D. Romeo ${ }^{3}$, A. Alecci ${ }^{4}$, S. Franciolini ${ }^{5}$, A. Campani ${ }^{5}$, C.

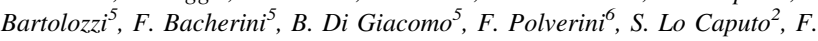
Mazzotta $^{2}$

${ }^{1}$ LILA-Toscana, Florence, Italy; ${ }^{2}$ UOC Malattie Infettive-Azienda Sanitaria Firenze, Florence, Italy, ${ }^{3}$ UO Medicina di Comunità-Azienda Sanitaria

Firenze, Florence, Italy; ${ }^{4}$ Dipartimento di Patologia Clinica-Azienda Sanitaria Firenze, Florence, Italy; ${ }^{5}$ UO Assistenza Infermieristica Territoriale-Azienda Sanitaria Firenze, Florence, Italy; ${ }^{6}$ Dipartimento di Statistica-Università di Firenze, Florence, Italy

The great progress in antiretroviral therapy have dramatically changed the evolution of HIV disease in the general population contributing to perceive the problem as solved: but the HIV epidemic continues to be present. The absence of effective public awareness campaigns and information on how to prevent infection, have led to a reduction in the number of HIV tests carried out. In order to promote information-educational intervention in Florence was designed a study to raise awareness and offer HIV testing to the general population. This epidemiological survey is designed to provide information on the prevalence of HIV in the Florence area. Assuming a prevalence of $1 / 1,000$ in this area you need to performed 10,000 tests. The project began in March 2010 with an information campaign on 'HIV with material and brochures available in the main venues of the population, in the sites of medical general practices and in the collection centers where you can have the HIV test. The second phase of the study began in April 2010 after a period of training of nurses working in the collecting centres in Florence with the offer of the HIV test to all those who perform a general blood examinations. Blood samples collected were processed in the laboratory of serology in SM Annunziata Hospital in Florence. The HIV test is made after signing the informed consent from the patient is free of charge, in respect of privacy and if the patient so wishes, with full anonymity. The target population of this study is mainly composed of adults, were deliberately excluded the infectious diseases clinics and those of SERT in order to avoid selection bias. The time of the collection will continue until you reach the amount required but not later than October 2011. At December 31, 2010 patients who have joined the initiative have been 4,539. Only one patient resulted HIV positive and he has been sent to the Infectious Diseases Unit of SM Annunziata Hospital, where he began the routine specialist checks. The relevance of such awareness campaigns to increase the offer to the test is of great importance not only in the aspect of health education, but also in the early diagnosis of HIV-infected patients by ensuring an appropriate treatment.

\section{PO 89}

\section{TOWARD IMPROVEMENTS IN HIV EPIDEMIOLOGY}

M. Prayer Galletti ${ }^{* 1}$, S. Pacini ${ }^{2}$, G. Morucci ${ }^{2}$, H.H. Bauer

${ }^{I}$ Department of Experimental Pathology and Oncology, University of Firenze, Florence, Italy; ${ }^{2}$ Department of Anatomy, Histology and Forensic Medicine, University of Firenze, Florence, Italy; ${ }^{3}$ Virginia Polytechnic Institute \& State University, Blacksburg, VA, USA

Considerable efforts are expended for prevention of HIV infections and for treatment of HIV-positive individuals, and it is widely agreed that improvements in both areas would be highly desirable. Observing and understanding the epidemiology of HIV are centrally necessary for the design of strategies for both prevention and for treatment. The way to improvement is to focus not on successes but on gaps to be filled or missteps to be corrected, so we discuss weaknesses of current practices and conundrums, why expected successes have not materialized. The fundamental uncertainty stems from the lack of a gold-standard HIV test. As a result, one cannot accurately compare HIV data from different global regions that use different testing protocols, for example, varying criteria for what constitutes a positive Western Blot or the availability or nonavailability of PCR or culture tests, or drawing inferences about HIV infection based on the Bangui definition of AIDS. In addition to uncertainty in cross-country comparisons, lack of the gold standard entails a fundamental inability to detect, analyze, and correct for false-negative and false-positive test-results by direct means rather than indirect inferences. Therefore, considerable effort would seem to be warranted to prepare pure samples of HIV for establishment of a true gold-standard HIV test. The weaknesses in testing practices may well account for at least some of the troubling conundrums and mutually contradictory data that seem inexplicable. These troubling conundrums include: conflicting estimates of HIV infections and of HIV-disease deaths from equally authoritative sources; apparently drastically different primary modes of transmission in different geographic regions (primarily among drug injectors in Russia and Eastern Europe, primarily among married couples in sub-Saharan Africa, primarily among gay men and drug addicts in the United States and Western Europe); extreme racial disparities in HIV infection, with Asians and Asian Americans consistently less affected, by about one-third, than white Americans, while black Americans are affected by as much as an order of magnitude more than white Americans. Testing uncertainties doubtless also contribute to the confusion as to whether certain conditions (e.g. lipodystrophy or nephropathy) should be described as HIV-associated or as AIDS-associated. Although it is the timehonoured practice in science that such anomalies or conundrums are quarantined in the expectation that progress will eventually resolve them without research focused directly at the anomalies, it would seem in the case of HIV/AIDS that specific efforts would be worth pursuing to resolve at least some of these conundrums, because a better understanding would improve epidemiological data and understanding and help toward the design of better strategies for prevention and treatment.

\section{PO 90}

META-ANALYSIS AND UPDATE ON THE GENERAL AIDS EPIDEMICS PREDICTED FOR AFRICA

P.H. Duesberg ${ }^{1}$, D. Mandrioli ${ }^{1}$, A. McCormack ${ }^{\text {I }}$, J.M. Nicholson ${ }^{2}$, C. Del

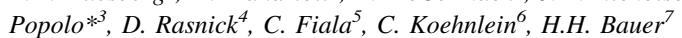

${ }^{1}$ University of California, Berkeley, USA $;{ }^{2}$ Virginia Tech, Blacksburg, USA; ${ }^{3}$ Department of Anatomy, Histology and Forensic Medicine, Florence, Italy;

${ }^{4}$ Oakland, California, USA; ${ }^{5}$ Gynmed Ambulatorium, Vienna, Austria;

${ }^{6}$ Internistische Praxis, Kiel, Germany; ${ }^{7}$ Virginia Tech, Blacksburg, USA

Since the discoveries of a presumably new AIDS virus in 1984 and of millions of asymptomatic carriers in subsequent years, no general AIDS epidemic has occurred in the U.S., Europe, South America and Asia by 2010. Recently, however, Chigwedere et al. "estimated" that between the years 2000 and 2005, the new AIDS virus, now called Human Immunodeficiency Virus (HIV), had killed 1.8 million South Africans at a steady rate of 300,000 per year, based on information from the World Health Organization (WHO) (J Acquir Immune Defic Syndr, 2008). Here we investigate the 
evidence for these claims in view of the paradoxes that (1) HIV would cause a huge epidemic in Africa, but not in any other continent despite global prevalence since 1985, and that (2) it would cause a steady rather than a classical bell-shaped epidemic, selflimited by immunity like all other new pathogenic viruses. Surprisingly, we found that the WHO does not even list any South African AIDS case from 1996 until 2007, and that Statistics South Africa attributed only about 10,000 deaths per year to HIV between 2000 and 2005, and thus 30-fold less than those reported by Chigwedere et al. In a further effort to find independent evidence for the reportedly new AIDS epidemic, we searched for losses of lives in South African population growth curves. Surprisingly, we found that South Africa had increased by 3 million between 2000 and 2005 extending a steady growth rate of 500,000 per year, based on statistics from South Africa, the US and the World Bank. This gain was an integral part of a monotonic growth trajectory from 29 million in 1980 before the AIDS era to 49 million in 2008. During the same time Uganda increased from 12 to 31 million, and Sub-Saharan Africa as a whole doubled from 400 to 800 million, despite high prevalence of antibodies against HIV. We deduce that the predicted epidemiological pattern of a new killing virus never showed up in Africa, and that HIV cannot be considered a killer virus from the demographic point of view.

\section{PO 91}

ACTIVE IN-THE-FIELD SURVEILLANCE REVEALS HIGH RATES OF HIV AND HEPATITIS INFECTION AMONG AN IMMIGRATE POPULATION

G. Stornaiuolo ${ }^{1}$, V. Cuniato ${ }^{2}$, G. Cuomo ${ }^{* 1}$, E. Nocera $^{2}$, G. Brancaccio $^{1}$, M. De Rosa $^{2}$, A. Pontarelli ${ }^{1}$, R.F. Natale ${ }^{2}$, G.B. Gaeta ${ }^{l}$

${ }^{I}$ UOC Malattie Infettive ed Epatiti Virali, Seconda Università degli studi di Napoli, Naples, Italy; ${ }^{2}$ Associazione di Volontariato "Jerry Essan Masslo", Castelvolturno, CE, Italy

Background and aims: Barriers to access medical care may underestimate the actual number of HIV, HBV, HCV positive subjects among immigrant population.

Methods: We recruited prospectively an immigrant population from the year 1999 to 2009 in a prevalently rural area (Castelvolturno, Naples) with massive, often irregular, immigration. During the first 5 years (1999-2004) an active recruitment was performed using a mobile unit moving through the area. In the second 5 years (2005-2009) only outpatients coming voluntary in the medical centre were recruited. Medical history was collected with the aid of cultural mediators using a pre-coded questionnaire. Each subject was tested for $\mathrm{HBsAg}$, anti-HBs, anti-HBc, $\mathrm{HBeAg}$, anti-HBe, anti-HCV, antiHIV.

Results: 2,681 subjects were observed (945 and 1736 in the two study periods), having a median length of stay of 3 years. Median age was 31 years (range 2-75), 52.8\% were males, $82.3 \%$ came from SubSaharan Africa; $64.6 \%$ had a middle-high school education level; $13.9 \%$ of women were sex workers. 129 subjects (5\%) were HIV Ab positive. HBV coinfection was present in 17 patients and HCV coinfection in 9 patients. 206 subjects were HBsAg positive $(7.6 \%)$, $84(3.6 \%)$ anti-HCV positive. 84 patients $(3.1 \%)$ were drugs addicted, $436(16.3 \%)$ were alcohol abusers. Anti-HBs were present in $28.2 \%$. The prevalences of $\mathrm{HBsAg}$ and anti-HCV remained constant throughout the study period while HIV significantly decreased. HIV patients were more frequently women $(\mathrm{p}=0.0001)$, sex workers $(\mathrm{p}=0.0006)$ and actively recruited $(\mathrm{p}=0.0001)$ than HBV and $\mathrm{HCV}$ patients. $\mathrm{HCV}$ patients were significantly older than $\mathrm{HBV}$ patients $(p=0.0018)$, and were more frequently drug and alcohol addicted $(\mathrm{p}=0.0001$ and 0.003 , respectively At multivariate analysis, HIV infection was independently associated to female gender $(p=0.01)$, drug addiction $(p=0.008)$, and the active way of recruitment $(p=0.04), \mathrm{HBV}$ infection to male gender $(\mathrm{p}=0.0001)$ and HCV to drug addiction $(\mathrm{p}=0.0001)$. Among sex workers about $25 \%$ of the subjects referred not to use condom or its sporadic use, but there were no differences in HIV prevalence as compared with those reporting a systematic use of condom.

Conclusions: An active recruitment can demonstrate a substantial prevalence of HIV and hepatitis infections. We can expect an escalation of the economic costs associated to chronic liver disease and HIV management and to educational and prevention campaigns.

\section{PO 92}

A WANDERING BUS FOR THE PREVENTION OF HIV AND

SEXUALLY TRANSMITTED DISEASES: THE PROJECT

"PARLIAMONETOUR" (LET'S TALK TOUR) OF THE COTUGNO HOSPITAL

A. Franco ${ }^{1}$, E. Guidetti ${ }^{1}$, M. Figoni ${ }^{I}$, C. Dell'Isola ${ }^{I}$

for "PARLIAMONETOUR" Group co-authors, A. Giordano ${ }^{1}$

${ }^{I}$ Monaldi-Cotugno-CTO Hospital, Naples, Italy

Introduction: The Cotugno Hospital in Naples since some years has been extending its interests beyond the specific mission (diagnosis and treatment of infectious diseases); in view of this, and to coincide with World AIDS Day on 1 December 2010, the same has started throughout Naples a project of information and prevention about sexually transmitted diseases (STDs), focusing especially on HIV, because prevention represents the most important investment in public health.

Materials and methods: The organization has provided for the involvement of all the ten municipalities of Naples, with the help of an equipped bus travelling (Figure) from 29 of November to December 12 2010. Several stops has been programmed nearby secondary schools to promote encounters with some experts (specialists of infectious disease, psychiatrists), also by answering to dedicated interviews (7 multiple choice quizzes on HIV infection, using a spreadsheet on-line). The operational plan also envisioned the distribution on the same bus of brochures on both HIV infection and the services of the hospital and the presentation of the project for distance learning already made in 2009 by a multidisciplinary team of Cotugno, that offered a full explanation on AIDS and STD to students through the use of a platform for e-learning on the company website ( http://www.ospedalecotugno.it). The participants at the project received a T-shirt dedicated to the event. The ambitious proposal called "PARLIAMONETOUR" has been designed using an ad hoc logo and a slogan already forwarded by the "Forum of Public Administration 2010" (... health is contagious) and found the adhesion of some sponsors.

Results: The project has involved in 14 days of activity 54 medical trainers (26 from Cotugno Hospital and 28 from public and private hospitals), and 3 facilitators and assistants; the overall respondents were 2,686 $(1,385 \mathrm{M}, 1,301 \mathrm{~F})$, mostly young people and adolescents $(1,712<20$ years), with a good instructional level (1,461 with a college or university education). An evaluation summary of the seven item multiple-choice questions (some of whom had only intended to 
"capture" what was the current perception of the HIV problem in our society), showed how most people think that HIV can be defeated, even though there still is a misinformation about transmission modality, especially about sexual behaviour.

Discussion: The preliminary results do not yet permit an overall assessment of the initiative, however, there seems laudable in intent and able to establish a first, positive contact between an highly specialized Hospital and the citizen.

Appendix: the following doctors are members of "PARLIAMONETOUR" Group and co-authors (*), Attanasio V., Carannante N., D’Abbraccio M., De Marco M., Gargiulo M., Iannece M.D., Liberti A., Marocco A., Martucci F., Matteis B., Maturo N., Miniero M., Morelli G., Nardini G., Parrella G., Parrella R., Perrella A., Pomicino A., Precchia E., Sardo M., Somma P., Viglietti R., Viparelli G.

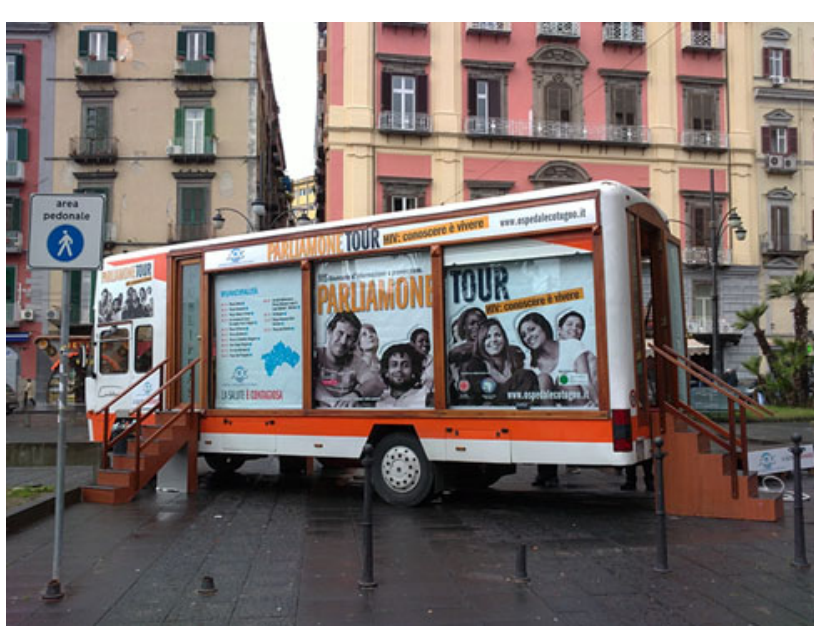

